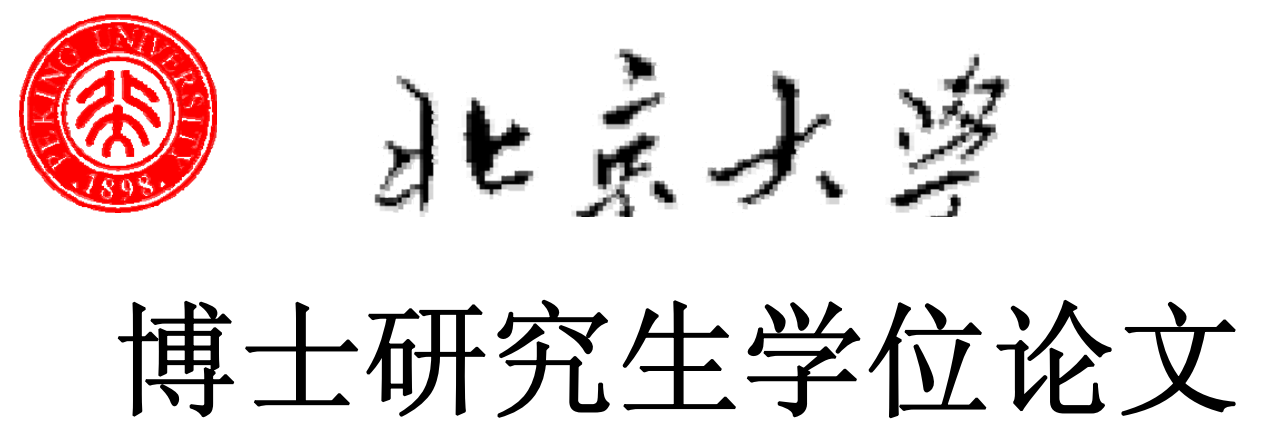

题目: 衍射辐射电子束束斑测量

及康普顿激光系统

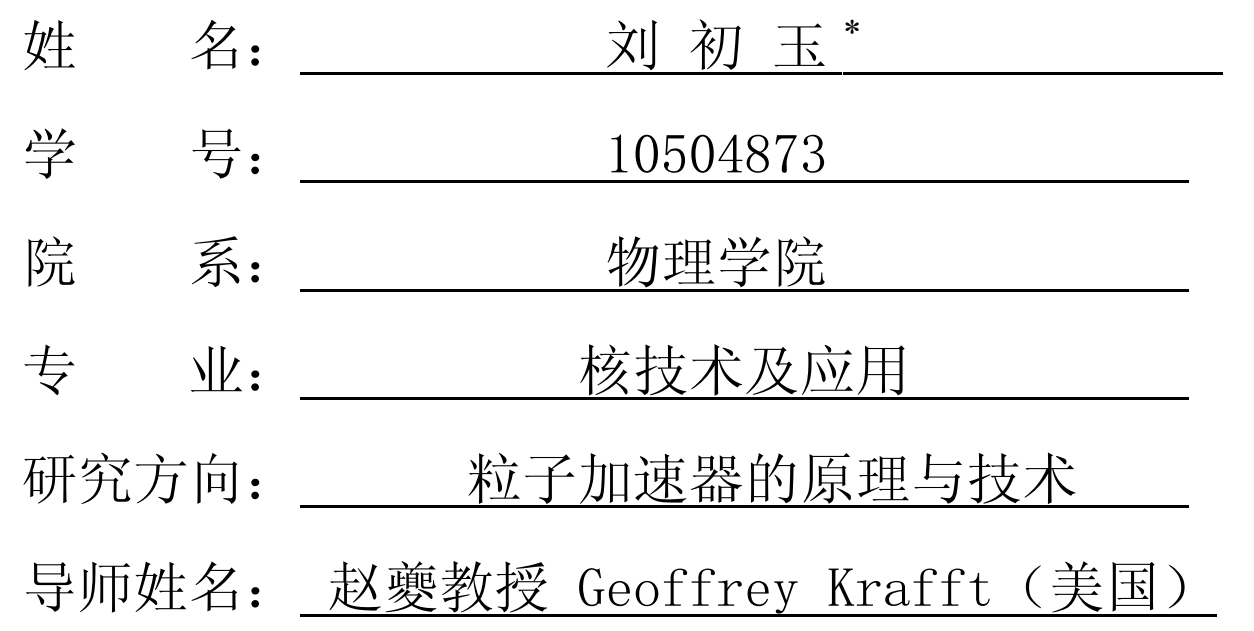

$$
\text { 二 } 00 \text { 九年六月 }
$$





\section{版权声明}

任何收存和保管本论文各种版本的单位和个人, 未经本论文作者同意, 不得 将本论文转借他人, 亦不得随意复制、抄录、拍照或以任何方式传播。否则, 引 起有碍作者著作权之问题, 将可能承担法律责任。 



\section{摘 要}

束流诊断是现代加速器系统中必备的重要组成部分, 其主要作用是对指定位 置的带电粒子束的能量、能散、发射度、电荷量、束斑大小及形状等参数进行测 量, 为加速器及束流传输系统的操作提供依据, 是进行束流动力学研究不可缺少 的手段。不同的诊断手段原理不尽相同，有的基于带电粒子的电作用，如法拉第 杯、电离室; 有的基于带电粒子的磁作用, 如束流变压器 (Beam Transformers)、 环形探针 (Pick-ups); 有些手段利用粒子与物质的核物理及基本粒子相互作用, 如束流损失监测仪、电子极化仪; 还有基于带电粒子的光辐射, 如渡越辐射、同 步辐射监测仪及本论文的第一部分工作一一衍射辐射 (Diffraction radiation); 以 及基于带电粒子与光子的相互作用, 如激光线扫描及本论文的第二部分工作一 康普顿电子极化仪。各种各样的诊断方法测量束流的各种参数, 作为监测、调节 机器和控制束流的重要依据。

最近和未来几十年内, 加速器发展的重点方向是高能对撞实验 (ILC, CLIC) 及第四代光源 (Euro-XFEL, LCLS, Spring 8-FEL)。这些新一代加速器需要更小 的束斑、更高的稳定性和束流品质。与现有的直线加速器相比较, 这些加速器在 各方面的性能都有很大的提高, 如水平方向束斑缩小 10 倍以上、坚直方向束斑 缩小几十倍及峰值电流强度提高几至几十倍。再加上在加速器的一些特殊位置对 于束流有着更严格的要求, 如对撞机的对撞点和自由电子激光的扭摆器。这些加 速器性能参数的提高直接加大了束流诊断的难度, 很多情况下拦截测量手段不再 适用, 而现有的非拦截方法无法适用于加速器的直线段部分或不能满足精度的要 求。加速器技术的发展迫切需要非拦截测量手段的同步发展, 提高诊断手段的性 能从而满足现在和未来加速器的发展需求。衍射辐射和康普顿散射是其中很有发 展潜力的测量手段, 它们的非拦截特点最大程度上减小了诊断元件对于束流传输 的影响以及测量仪器自身的损伤。本论文的工作分为两部分, 分别是在 CEBAF 机器上采用衍射辐射装置测量电子束束斑和基于康普顿散射测量电子极化的激 光系统。

衍射辐射和渡越辐射有很多共同点, 都是带电粒子电场与靶相互作用的结 果。尽管关于衍射辐射的理论研究出现得比较早, 但其实验研究及实际测量应用 在近几年内才出现。在 CEBAF 机器上利用衍射辐射实现电子束束斑的测量是衍 
射辐射应用的一个重要进步: 首先, 该实验的成功证明了衍射辐射是一个有效的 非拦截测量方法; 其次, 衍射辐射分布尺寸与实际束斑大小简单的线性关系大大 提高了衍射辐射测量的可靠性; 另外, 第一次在实验上测量了衍射辐射极化分量 分布并成功解释了辐射干扰的来源, 提出了排除干扰的方案; 分析了水平极化分 量非对称性并提出相应的解决办法; 最后, 第一次在实验上观察到了很强的极化 波片效应。

所有利用带电粒子与光子相互作用的测量手段, 如激光线扫描仪、激光干涉 测量仪和康普顿电子极化仪, 都是建立在稳定高品质激光系统的基础之上。论文 第二部分的内容是建立一套用于康普顿散射的激光测试装置。该系统在 $1064 \mathrm{~nm}$ 激光平台上成功结合了二极管技术和光纤技术; 利用了增益开关二极管技术, 得 到了具有灵活重复率和皮秒量级束团长度的激光源; 成功实现了全光纤脉冲选择 技术，拓展了该系统的应用空间；以及成功实现了一项新的脉冲生成技术，提升 了脉冲序列的相干性。这些就是论文第二部分的创新点。

此外, 作者还对激光整形技术进行了一些研究, 提出了一种简洁的设计办法, 并设计了大口径、单透镜整形器, 降低了激光整形中的衍射效应, 减少了表面损 耗。该研究成果正在专利申请阶段。

关键词: 束流诊断, 非拦截测量, 衍射辐射, 反康普顿散射, 增益开关二极管, 光纤激光 


\title{
Beam size measurement by optical diffraction radiation and Laser system for Compton polarimeter
}

\author{
Chuyu Liu (Nuclear Technology and application) \\ Directed by Prof. Zhao Kui and Dr. Geoffrey Krafft
}

\begin{abstract}
Beam diagnostics is an essential constituent of any accelerator, so that it is named as "organs of sense" or "eyes of the accelerator." Beam diagnostics is a rich field. A great variety of physical effects or physical principles are made use of in this field. Some devices are based on electro-magnetic influence by moving charges, such as faraday cups, beam transformers, pick-ups; Some are related to Coulomb interaction of charged particles with matter, such as scintillators, viewing screens, ionization chambers; Nuclear or elementary particle physics interactions happen in some other devices, like beam loss monitors, polarimeters, luminosity monitors; Some measure photons emitted by moving charges, such as transition radiation, synchrotron radiation monitors and diffraction radiation-which is the topic of the first part of this thesis; Also, some make use of interaction of particles with photons, such as laser wire and Compton polarimeters-which is the second part of my thesis. Diagnostics let us perceive what properties a beam has and how it behaves in a machine, give us guideline for commissioning, controlling the machine and indispensable parameters vital to physics experiments.

In the next two decades, the research highlight will be colliders (TESLA, CLIC, JLC) and fourth-generation light sources (TESLA FEL, LCLS, Spring 8 FEL) based on linear accelerator. These machines require a new generation of accelerator with smaller beam, better stability and greater efficiency. Compared with those existing linear accelerators, the performance of next generation linear accelerator will be
\end{abstract}


doubled in all aspects, such as 10 times smaller horizontal beam size, more than 10 times smaller vertical beam size and a few or more times higher peak power. Furthermore, some special positions in the accelerator have even more stringent requirements, such as the interaction point of colliders and wigglor of free electron lasers. Higher performance of these accelerators increases the difficulty of diagnostics. For most cases, intercepting measurements are no longer acceptable, and nonintercepting method like synchrotron radiation monitor can not be applied to linear accelerators. The development of accelerator technology asks for simutanous diagnostics innovations, to expand the performance of diagnostic tools to meet the requirements of the next generation accelerators. Diffraction radiation and inverse Compton scattering are two of the most promising techniques, their nonintercepting nature avoids perturbance to the beam and damage to the instrumentation. This thesis is divided into two parts, beam size measurement by optical diffraction radiation and Laser system for Compton polarimeter.

Diffraction radiation, produced by the interaction between the electric field of charged particles and the target, is related to transition radiation. Even though the theory of diffraction radiation has been discussed since 1960s, there are only a few experimental studies in recent years. The successful beam size measurement by optical diffraction radiation at CEBAF machine is a milestone: First of all, we have successfully demonstrated diffraction radiation as an effective nonintercepting diagnostics; Secondly, the simple linear relationship between the diffraction radiation image size and the actual beam size improves the reliability of ODR measurements; And, we measured the polarized components of diffraction radiation for the first time and I analyzed the contribution from edge radiation to diffraction radiation.

Keywords: Beam diagnostics, Non-intercepting measurement, Diffraction radiation, Inverse Compton scattering, Gain-switched diode, Fiber laser 


\section{目录}

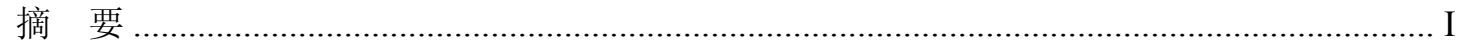

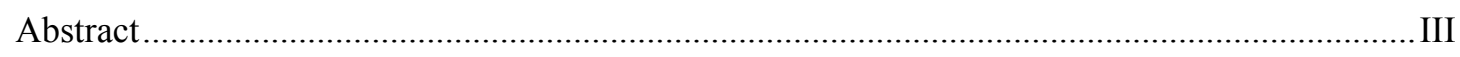

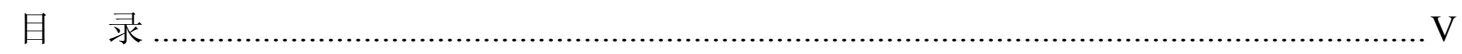

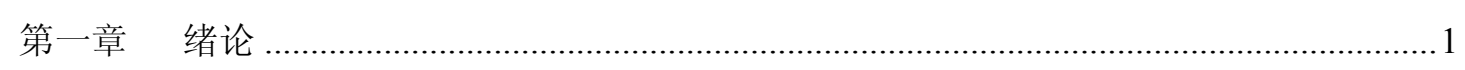

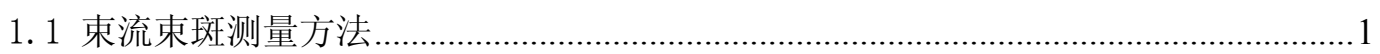

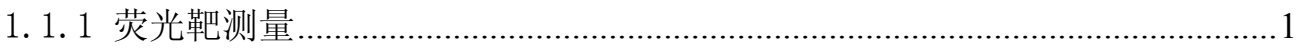

1.1 .2 坚琴测量

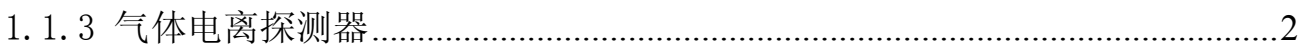

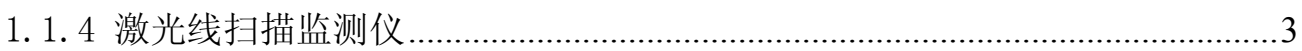

1.1 .5 激光干涉仪

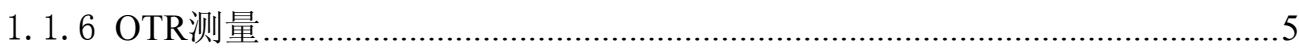

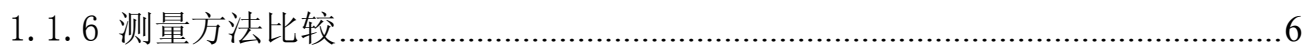

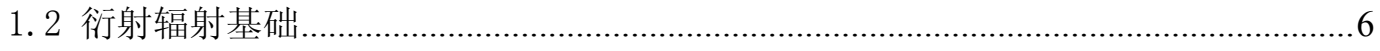

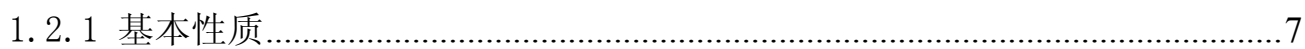

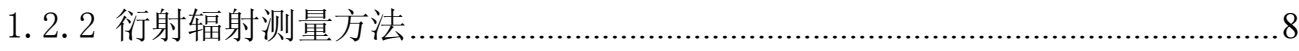

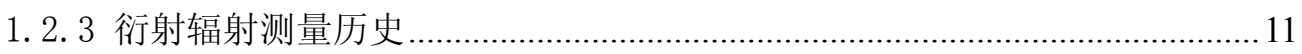

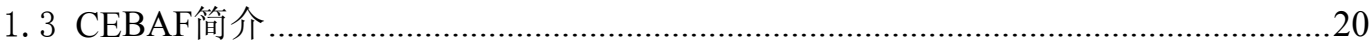

1.4 为什么CEBAF需要衍射辐射测量 ………………………………………………………......22

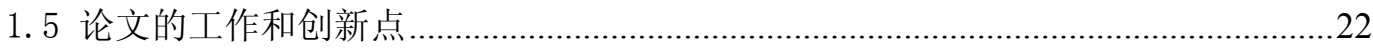

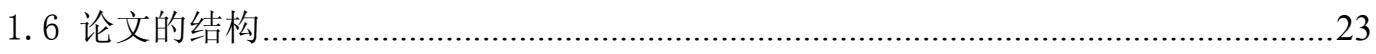

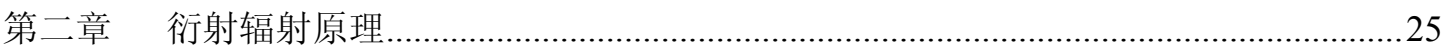

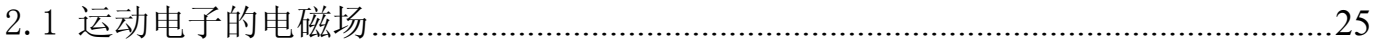

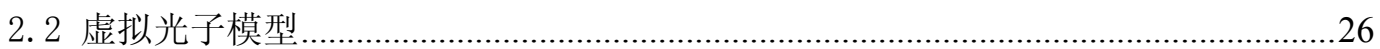

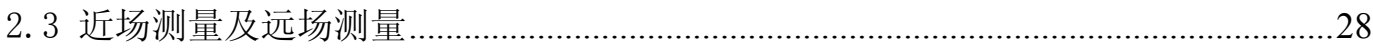

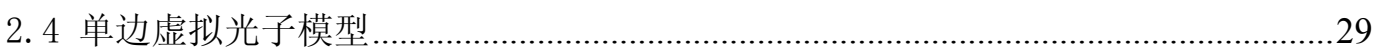

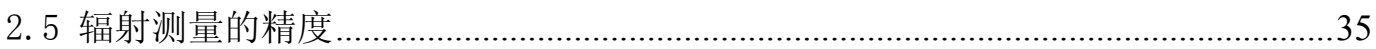

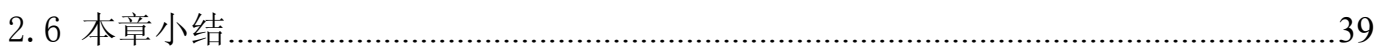




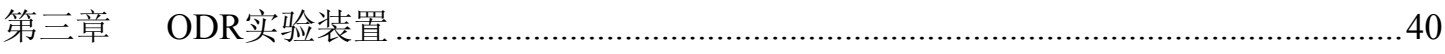

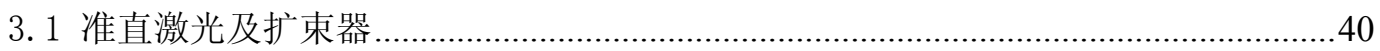

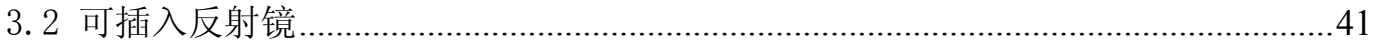

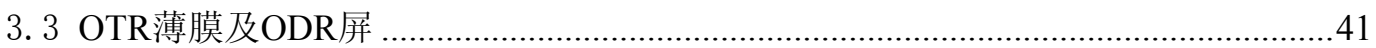

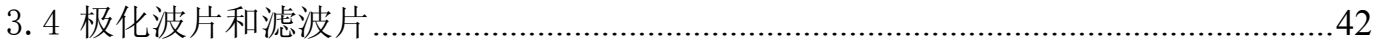

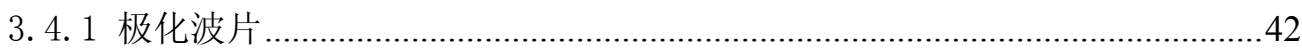

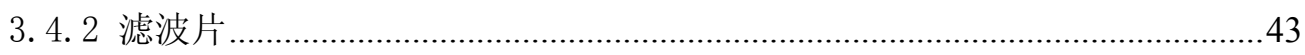

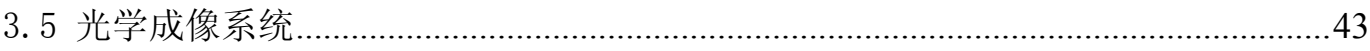

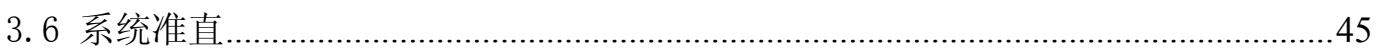

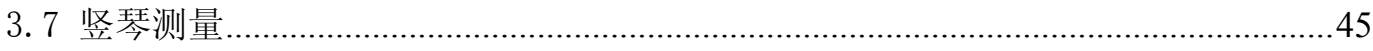

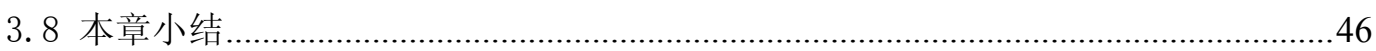

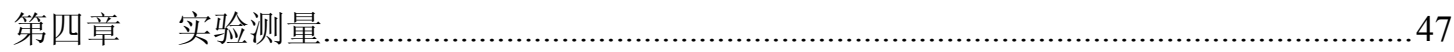

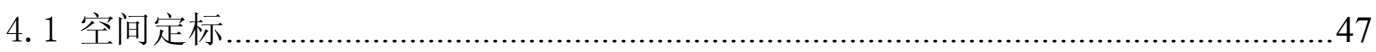

4.2 坚琴测量

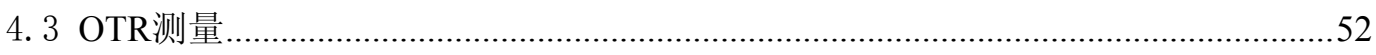

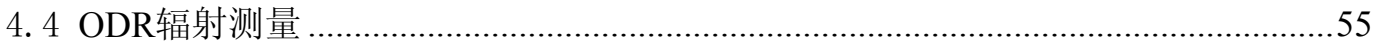

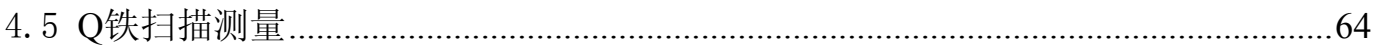

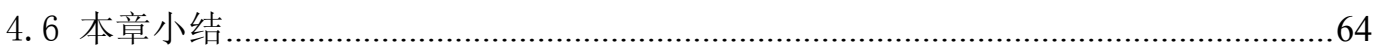

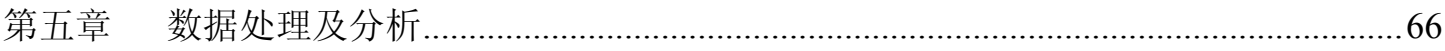

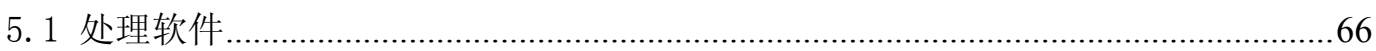

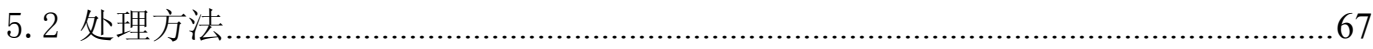

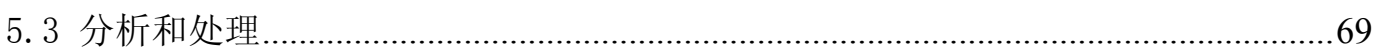

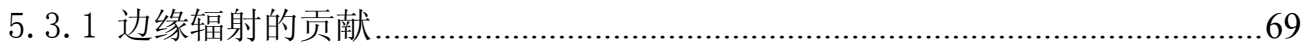

5. 3. 2 针对边缘辐射的提案……………………………………………………….......

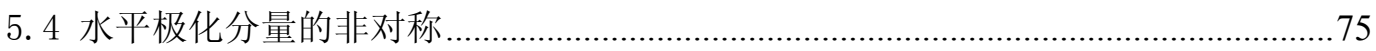

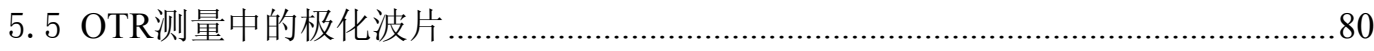

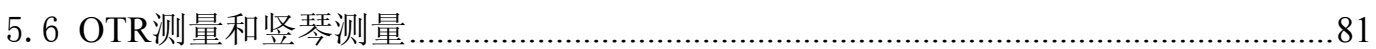

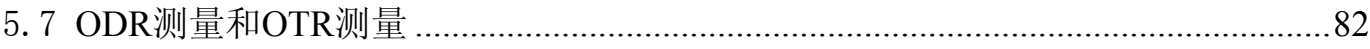

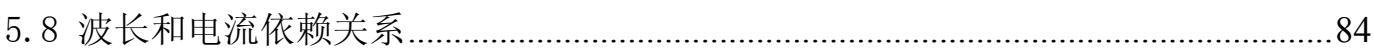

5.9 本章小结 
第六章 康普顿激光系统简介.

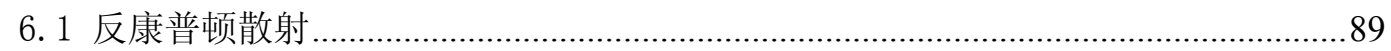

6.2 激光参数要求

6. 3 Hall C康普顿电子极化仪及激光要求 ……………………………………………….........90

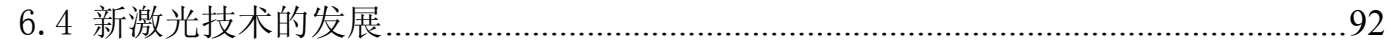

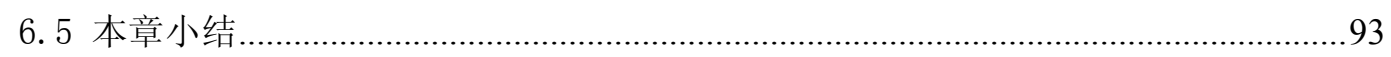

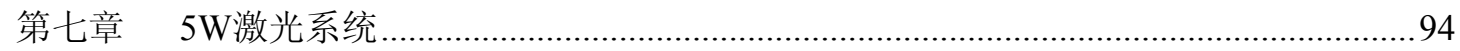

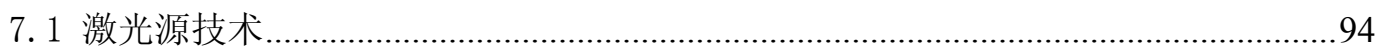

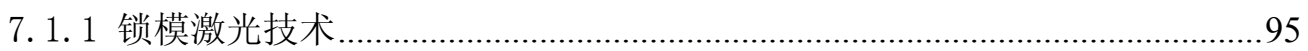

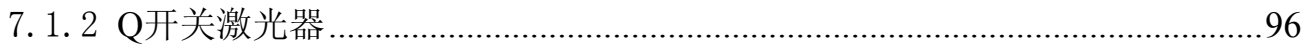

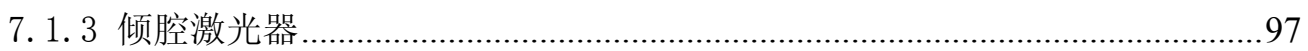

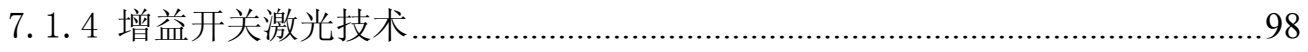

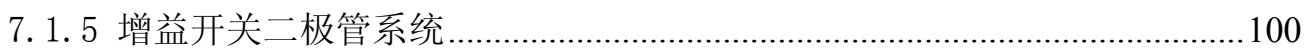

7. 1.6 光学反馈

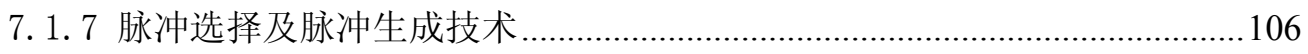

7.2 光学放大器

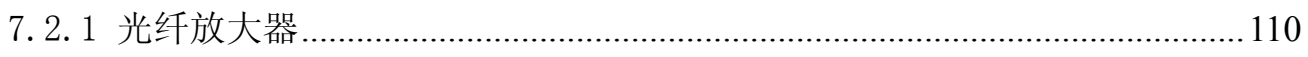

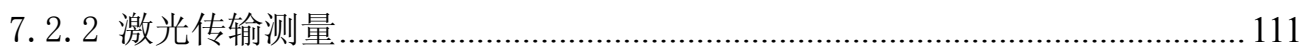

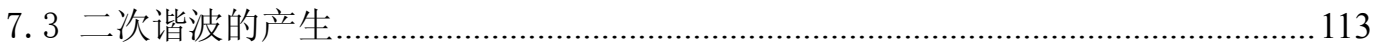

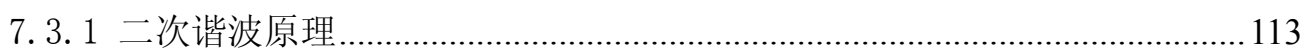

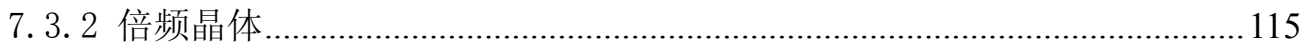

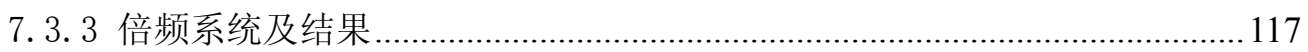

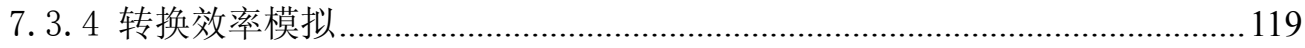

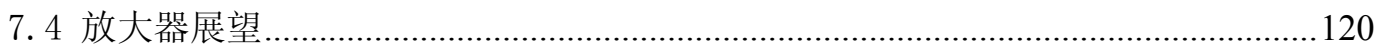

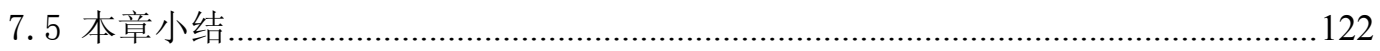

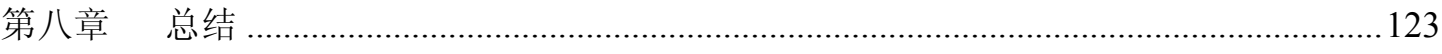

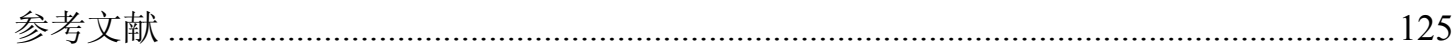

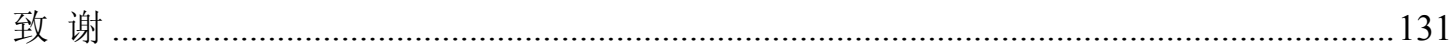





\section{第一章 绪论}

束斑大小的测量是加速器诊断中最基本的一个环节, 从加速器最初的发展到 现在, 束斑大小一直是最受关注的重要参数。无论加速器的规模大小及束流的用 途, 束斑大小测量的重要性都无法改变。束斑的测量可以监测束流品质, 检验光 学元件的聚散焦特性, 间接地测量束线传输矩阵, 测量束流的相空间参数, 作为 不同束线段匹配的依据[1], 还可以测量束流发射度、色散函数等, 对束流品质 和机器的诊断具有重要的意义[2]。

本章重点在于分析下面几个问题: 为什么需要发展新的测量手段? 衍射辐射 能测量那些参数, 为什么选择了它? 为什么要在 CEBAF 进行衍射辐射测量? 以 及衍射辐射测量都有了那些工作? 从这些基本问题出发, 本章将概括现有的束斑 测量办法, 比较它们的优缺点, 以及阐述发展非拦截束斑（ 100um）测量办法 的重要意义和急迫性; 然后介绍衍射辐射的基本概念, 该诊断方法能测量的束流 参数, 及国际上已经开展的衍射辐射测量工作; 然后介绍杰佛逊实验室 CEBAF 机器的基本概况以及在该机器上进行衍射辐射测量的必要性及其特殊的意义。最 后, 介绍本论文的选题和特点, 以及论文的结构安排。

\section{1 束流束斑测量方法}

随着加速器技术的进步, 束斑测量技术也经历了长足的发展, 测量方式从最 初的拦截式的荧光靶测量到现在的非拦截式测量, 测量精度从百十微米提高到现 在的亚纳米量级。虽然随着强流加速器的发展和束流性能的普遍提高, 束斑测量 要求用非拦截式取代拦截式测量方法, 同时对测量精度的要求也越来越高。然而, 每一种测量方式都有其优缺点和使用范围, 下面的内容将对现有的测量办法进行 一个概括和比较, 找到迫切需要发展的测量范围, 确定新技术的发展方向。

\section{1.1 荧光靶测量}

苂光泛指材料吸收光子后释放波长更长光子的现象, 在光学上被称作红移。 吸收和释放光子的能量差表现为分子振动即热现象。磷光是一种特殊的荧光, 磷 光物质在电子或紫外光照射后会持续发光，长达几小时。二者并无严格的界定， 
对于加速器束流截面测量中所用的靶发光材料的称呼, 苂光材料和磷光材料是混 用的。最常用的是钇铝石榴石 (YAG) 靶[3], 当电子束经过靶时部分被吸收, 然后 YAG 靶发出绿光。在非饱和条件下, 绿光强度在靶上分布正比于电子束截 面电荷强度, 可以直接测量束斑大小和强度分布, 常用于低能束流的测量[4]。

\section{1 .2 坚琴测量}

坚琴测量（Harps Measurement）[5]也被称为多丝扫描仪（Wire Scanner）测 量。它是可以横向扫描带电粒子束团的多丝线（铇丝或碳纤维）设备。扫描时粒 子与细丝相互作用会产生散射光子或二次电子, 通过记录它们的强度与细丝的位 置关系就可以得到束流截面分布。坚琴测量的精度由细丝的直径和扫描的精度共 同决定。图 1.1 是一个常见坚琴的示意图[6]:

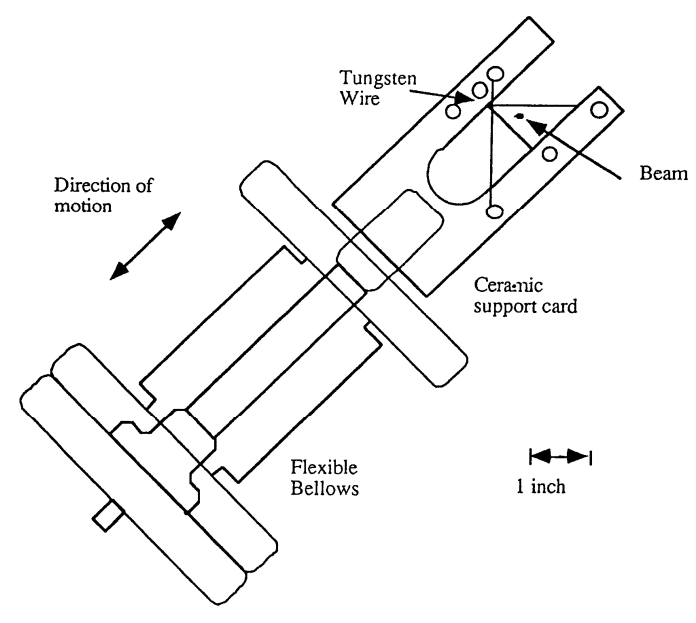

图 1.1 坚琴装置示意图

通常细丝装置和水平方向成 $45^{\circ}$ 角, 并沿该方向扫描束流截面。水平细丝可以 测量束流坚直方向分布, 坚直细丝则可以测量束流水平方向分布。

\subsection{3 气体电离探测器}

利用电动力学的知识可知, 高速运动的电子束的电场主要集中在横向, 因此 其空间电荷力方向也在横向。当气体 (氦气或氩气) 被注入后, 位于电子径迹的 气体会被电子束所电离, 继而受到电子束空间电荷力的作用。以 $\mathrm{Ar}^{+}$为例, 电子 束电场的强弱决定于电子束斑大小和离子位置, 电子束电场强弱然后决定了气体 离子所受作用力的大小, 该作用力又决定了离子到达探测器的飞行时间的长短, 
因此, 对气体离子飞行时间的测量可以提供束流束斑信息, 而且, 进一步的角度 分布测量能告诉测量者束流横向截面的比例关系。

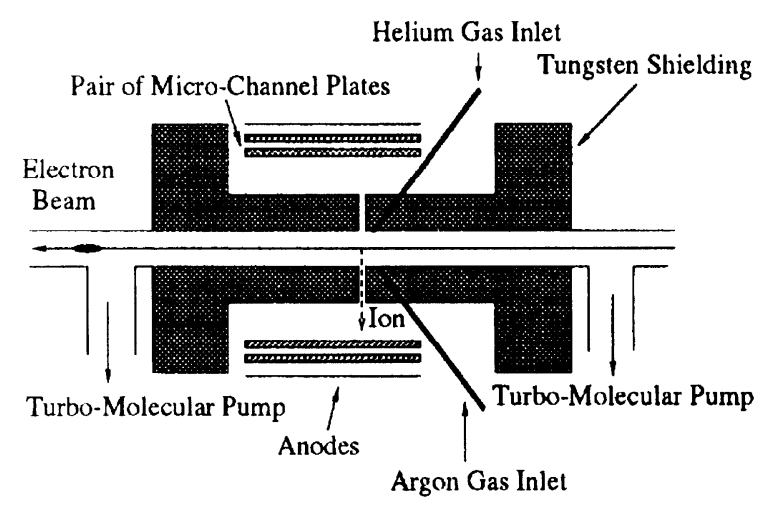

图 1.2 气体电离探测器

如 图 1.2 所示[7], 气体电离室的探测元件是围绕束管的微通道板 (Microchannel Plates), 狭缝用于气体注入和气体离子的通道, 狭缝和微通道板之间的电压差会 进一步加速气体离子。该方法被成功应用于FFTB坚直 $90 \mathrm{~nm}$ 束斑大小的测量。

气体电离探测器的优点在于: 对于电子束的能量不敏感; 本底很低。其缺点 是需要束团电荷和束团长度的信息而且数据后处理复杂。

\section{1 .4 激光线扫描监测仪}

激光线扫描监测仪 [8]基于类似于坚琴测量的原理, 只是使用了细的激光束来 代替金属丝线扫描。和电子束相互作用处的激光束腰是靠 F-P 激光腔[9]形成的, 同时 F-P 激光腔也大大地提高了激光功率。激光与电子束的相互作用在电子束径 迹的方向上产生高能光子, 通过测量散射光子和激光位置的关系, 然后在已知激 光截面分布的基础上通过反卷积得到电子束截面的分布。

该种监测仪一般位于偏转二极铁的上游, 目的是为了方便探测器测量沿电子 束方向的散射光子。取决于激光波长和电子束能量, 散射光子一般为 $\gamma$ 光子或 $x$ 射线, 所以探测器为常见的闪烁探测器。这种监测仪的工作重点在于小束腰高强 度激光系统的建立。激光波长一般取 $532 \mathrm{~nm}$ 或 $1064 \mathrm{~nm}$, 短波长有助于形成小束 腰。现有的激光器无法提供高重复率且高功率的激光, 其解决办法有两种: 一是 使用高重复率的锁模激光器加 F-P 激光腔, 该激光腔长需和锁模激光腔长相匹 配; 另外是使用窄频谱的连续激光加 F-P 激光腔。如果模式匹配和 F-P 激光腔长 
设置得好, 腔内被来回反射的激光会相干叠加而形成很高的腔内功率。

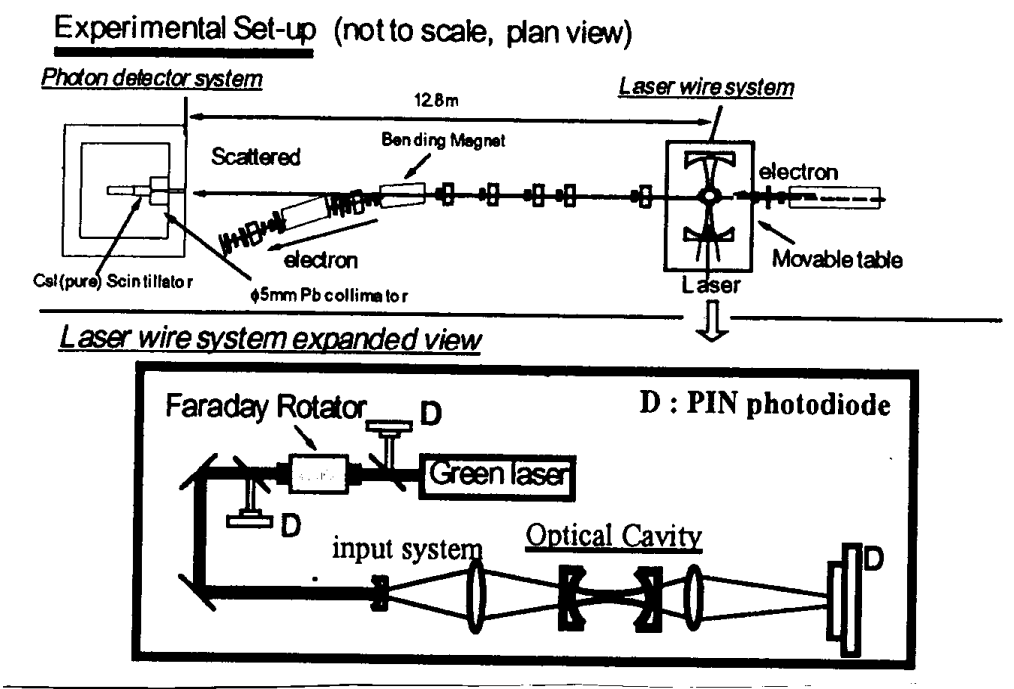

图 1.3 激光线扫描仪及其激光系统示意图

\section{1 .5 激光干涉仪}

激光干涉仪可以用于小到几个 $\mathrm{nm}$ 量级束斑的测量, 该技术已被成功应用于 SLAC FFTB[10]。下面是装置示意图:

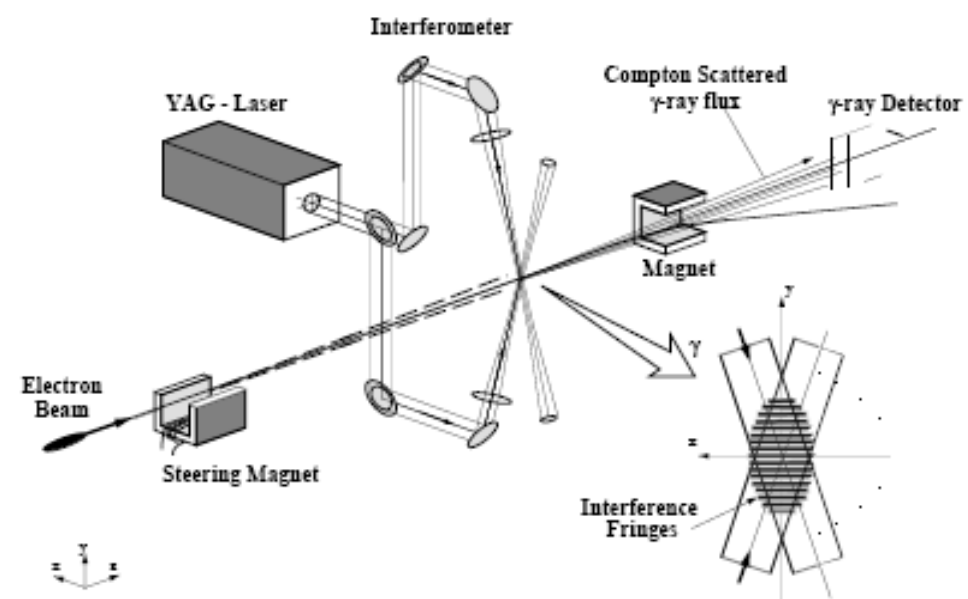

图 1.4 激光干涉仪示意图

激光束被等分成两路, 然后又以一定角度相对碰撞, 在两束激光重合处产生干涉 条纹。测量办法是将电子束沿激光干涉条纹扫描, 这时散射光子计数随之出现调 制，从对该调制的拟合可以推导束斑大小的信息。该干涉仪在测量中遇到的问题 主要是: 散射光子的低信噪比、激光位置的漂移、激光时间抖动和激光位置抖动。 F-P 激光腔的使用可以解决信噪比低和激光位置抖动的问题, 因为激光腔能够提 
高激光功率几个量级且直接决定了激光重合的位置即干涉条纹的位置。下面是运 用激光腔进行干涉测量的示意图[11]:

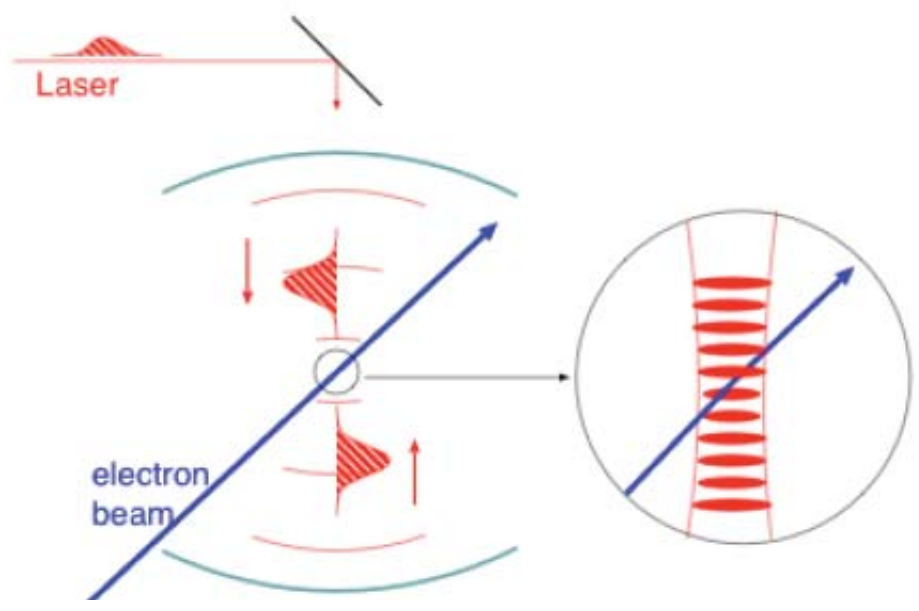

图 1.5 用于激光干涉仪的 F-P 激光腔

此时激光重复率应该是腔往返时间倒数的两倍。很明显, 这样设置的优势在于强 激光功率和两束激光的完美重合。

\section{1.6 OTR测量}

OTR 是带电粒子穿过不同介质截面时产生的波长在可见和红外的辐射。如图 所示[12], OTR (前向和后向辐射) 辐射都是以圆雉形状向外发射, 辐射最强位 于角度 $\theta=1 / \gamma$ [13]。为了方便一般对后向 OTR 辐射进行测量, 其强度正比于辐 射屏的反射率。辐射屏材料一般选用镀金属膜的薄膜材料, 一方面减小对于电子

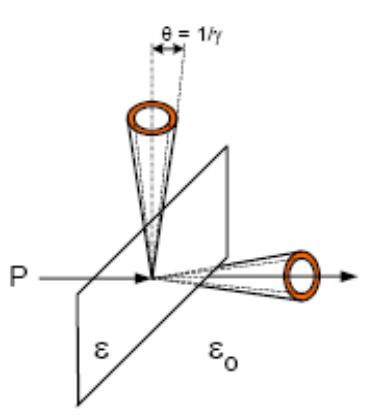

(a)

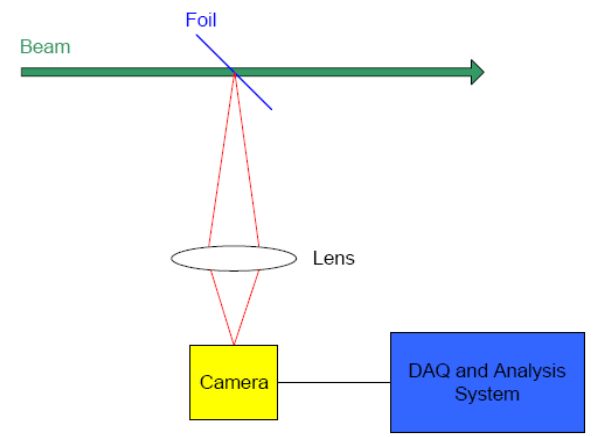

(b)

图 1.6 OTR 示意图:（a）OTR 辐射分布;（b） OTR 截面测量示意图 束的散射, 另一方面提高屏的镜面反射率。图中是对电子束截面测量的设置, 辐 射屏上强度位置分布 (正比于电子束截面电荷分布密度), 被光学元件成像在 
CCD 相机上, 通过定标（或反卷积）来得到电子束截面分布。

\section{1.6 测量方法比较}

以上给出了现有束斑大小测量手段的分别介绍, 每一种方式都有自己的优缺 点, 都有他们所适用的范围和具体情况。下面表格是在几个主要关心的问题上对 这些方法的一个总结:

表格 1.1 束斑测量方法的比较

\begin{tabular}{cccccc}
\hline 测量方法 & 是否拦截 & 是否要强流 & 精度 $(\mathrm{um})$ & 费用 & 占地 \\
\hline 苂光靶 & 是 & 否 & $\sim 10$ & 低 & 小 \\
坚琴测量 & 是 & 否 & $\sim 1$ & 低 & 小 \\
气体电离室 & 否 & 是 & $\sim 0.01$ & 高 & 小 \\
激光线扫描 & 否 & 是 & $\sim 0.3$ & 很高 & 大 \\
激光干涉仪 & 否 & 是 & $\sim 0.01$ & 很高 & 大 \\
OTR 测量 & 是 & 否 & $\sim 1$ & 低 & 小 \\
\hline
\end{tabular}

通过比较这些测量方法, 可以发现, 在几微米到上百微米的常见束斑范围内, 一般并不需要高精度但昂贵的测量方法如气体电离室、激光线扫描和激光干涉 仪; 而测量精度和费用都适中的测量方法如荧光靶、坚琴测量和 OTR 测量却需 要拦截束流。因此, 精度适中、价格低廉的非拦截束斑测量手段将会有很大的使 用空间。

\section{2 衍射辐射基础}

新一代深紫外及 $\mathrm{x}$ 射线高功率辐射装置, 如短波长自由电子激光, 都需要有 能产生高品质束流的直线加速器。这些高功率、小束斑和低发射度的束流装置给 束流诊断及控制带来了不小的挑战。普通的观察靶等拦截式测量手段不再适用于 这些装置。一些非拦截式的测量方法, 比如同步辐射监测仪, 又只能用于非直线 段部分。因此, 发展紧凑、非拦截式测量工具对于这些高功率机器的发展至关重 要。近几年, 衍射辐射测量成为非拦截测量方式的一个热门研究项目, 该测量方 式的特性使得其很有希望能成功应用于高品质束流直线加速器。 


\subsection{1 基本性质}

衍射辐射是带电粒子穿过孔隙或在不同介质截面附近通过时产生的辐射。孔 隙或介质边界上电荷分布在束流经过时出现突然变化, 这种变化会以辐射即衍射 辐射的形式释放出来[14]。如同渡越辐射一样, 衍射辐射也可以被理解为辐射靶 存在下的束流尾场 $[15,16]$ 。

衍射辐射最重要的参数, 即决定衍射辐射强度的参数是辐射因子, $a=\gamma \lambda / 2 \pi, \gamma$ 是洛伦兹因子, $\lambda$ 观察的辐射波长。辐射因子 $a$ 实质上是表征运 动电荷径向电场衰减特性的参数。将辐射屏离束流的最近距离表示为 $l$, 如果 $l \leq a$, 束流经过时将会在辐射屏上触发波长为 $\lambda$ 的可观的衍射辐射; 如果 $l \gg a$, 能产生的波长为 $\lambda$ 的衍射辐射将会很弱。可以理解, 有限大辐射屏上产生的渡越 辐射等于无限大辐射屏的渡越辐射与同形状孔径产生的衍射辐射的差值。

在辐射靶垂直于束流径迹的条件下，沿束流方向的衍射辐射被称作前向衍射 辐射; 沿束流反方向的衍射辐射则被称作为后向衍射辐射。后向 DR 和前向 DR 除了强度上的差别外本质上是一样的[17]。为了方便, 通常辐射靶会被安装成与 束流方向成 $45^{\circ}$ 角, 这样的设置下后向 DR 会以 $90^{\circ}$ 夹角垂直于束管出射, 以 此方便于辐射探测元件的布置。

衍射辐射可以通过远场（Fraunhofer Zone）观察，也可以进行近场（Fresnel Zone）观察 [18]。其远场的微分谱角分布和渡越辐射的分布类似。如同渡越辐射, 衍射辐射的角分布受束斑大小的影响, 因此可以利用这一性质进行束斑大小的测 量。然而, 该角分布同时也是束流发散角和束流位置的函数, 因此, 在能成功区 分这些影响因素的条件下，衍射辐射可以被利用为多束流参数测量工具[19]。

衍射辐射和渡越辐射一样都产生宽谱辐射, 如果所观测的辐射波长小于粒子 束团的长度, 该范围的辐射属于非相干辐射, 辐射强度正比于束团电荷量; 如果 所观测的辐射波长可比于或大于粒子束团的长度, 该范围的辐射属于相干辐射, 辐射强度正比于束团电荷量的平方。这一性质通常被应用于束团长度的测量中 $[20]$ 。在大多数的机器中, 束团长度都比横向尺寸要大。因此, 在短波长处对于 非相干辐射的测量能给予束斑大小、束流位置及发散角的信息; 而在长波长对于 相干辐射的测量则可以推导束团长度的信息[21]。

衍射辐射作为一种非拦截式测量手段, 不会对束流本身带来可观的扰动, 能 
够被应用于束线的任意位置, 而且可以用于测量多种束流参数, 比如束流位置、 束斑大小、能量、发射度和束团长度。理论上说, 产生衍射辐射的辐射屏可以做 成任意形状, 但带圆孔、狭缝的辐射屏和单边辐射靶是人们通常的选择。不同几 何形状辐射屏的选择决定了其所能测量的束流参数, 下面以圆孔辐射靶为例来分 析衍射辐射测量。

\subsection{2 衍射辐射测量方法}

如下图所示, 电子束穿过辐射屏上半径为 $r$ 的孔隙中心。假设辐射屏的尺寸 远大于辐射因子 $a$, 那么就可以忽略辐射屏的有限尺寸对于辐射的影响, 换句话 说可以将辐射屏处理为带半径为 $r$ 的孔隙的无限大平面。

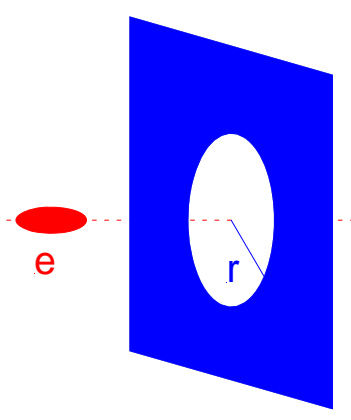

图 1.7 带圆形孔隙的金属屏作为衍射辐射靶

单电子所产生的衍射辐射角分布[14]可以表示为:

$$
d^{2} N_{\omega} / d \omega d \Omega=\frac{1}{137 \pi^{2}} \frac{4 \gamma^{2} x^{2}}{\left(1+x^{2}\right)^{2}}\left[J_{0}^{2}(R x)+\frac{\left\langle\rho^{2}\right\rangle}{a^{2}} J_{1}^{2}(R x)\right] \frac{\Delta \omega}{\omega}
$$

其中, $\left\langle\rho^{2}\right\rangle$ 是束流半径均方, $p=\left\langle\rho^{2}\right\rangle / a^{2}, \quad x=\gamma \theta, \quad R=2 \pi r / \gamma \lambda$ 。假设束流在 横向呈高斯分布, 考虑束团内粒子位置和散角分布, 电子束团产生的辐射角分布 为:

$$
\begin{aligned}
d^{2} N_{\omega} / d \omega d \Omega= & \frac{1}{137 \pi^{2}} \int_{-4 s}^{4 \varsigma} d \varsigma \frac{4 \gamma^{2}(x+\varsigma)^{2}}{\left(1+(x+\varsigma)^{2}\right)^{2}}\left[J_{0}^{2}(R(x+\varsigma))+\right. \\
& \left.\frac{\left\langle\rho^{2}\right\rangle}{a^{2}} J_{1}^{2}(R(x+\varsigma))\right] \frac{\Delta \omega}{\omega} \frac{1}{\sqrt{2 \pi} s} e^{-\varsigma^{2} / 2 s^{2}}
\end{aligned}
$$

从理论上来讲, 一共有三种办法可以得到束流位置的信息, 它们分别是: 强度角 分布测量、近场强度分布测量和辐射极化测量[19]。 
首先, 强度角分布受到束流在孔隙内位置的影响, 束流偏离孔隙中心将会导 致角分布的不对称，而且偏离越远，总的衍射辐射强度越强; 同样，衍射辐射强 度在孔隙周围分布也受到束流位置的影响, 辐射近场强度分布如同传统 BPM 电 信号一样可以作为位置测量的手段; 如果束流通过孔隙的中心, 衍射辐射的极化 特性就和渡越辐射一样是径向极化, 束流位置的变化会引起辐射极化的变化, 而 且这种变化是近似线性变化。

保持束流散角不变的情况下，辐射分布随束斑大小的变化如下:

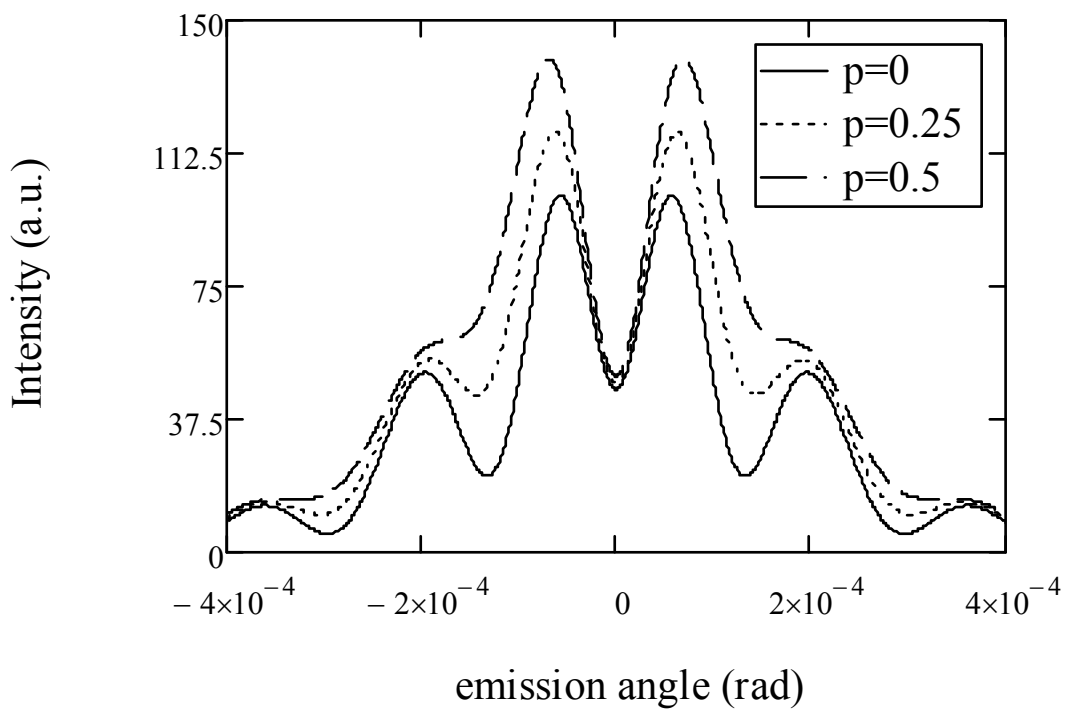

图 1.8 束斑大小对圆孔衍射辐射角分布的影响

如预期一样, 束斑大小的改变直接改变了束团电子离孔径的距离, 从而引起辐射 强度的变化, 而且束团越大辐射越强。其中 $p=0$ 表示线电荷的情形。可以理解, 角分布中峰值和谷底之比直接决定于束斑大小，因此，由该比值变化信息可以推 导束斑大小。

在保持束斑大小不变的条件下, 辐射分布随束流发散角的变化如图所示。可 见, 发散角的增加降低了辐射角分布的峰值且钝化了对比度。相比于束斑大小, 束流发散角是对比度更为敏感的参数, 因此圆孔辐射屏产生的衍射辐射也可以用 于发散角的测量。 


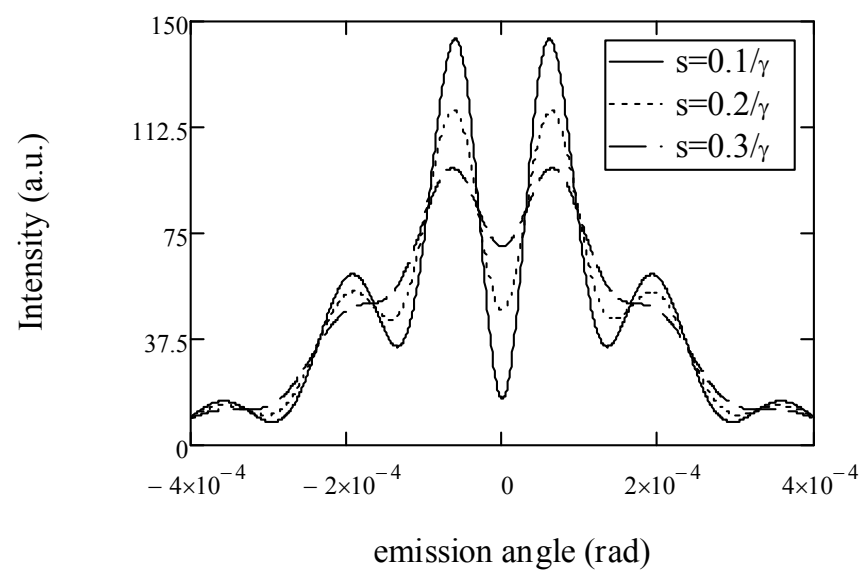

图 1.9 发散角对于圆孔衍射辐射角分布的影响

如果两个 ODR 辐射屏被放置在束线上间距近似为衍射辐射相干长度的两个 位置, 屏上产生的辐射将会出现相干, 所能观察到的角分布将会是单屏辐射角分 布上出现调制条纹。通过模拟, 调制条纹的位置就可以给出束流能量的信息。

衍射辐射的干涉[22]可以通过两种实验设置来实现，一是靶 1 上产生的前向 辐射和靶 2 上的后向辐射干涉, 另外就是两个辐射靶上的后向辐射发生干涉。下 面以第一种情况为例来分析可以测量的束流参数。和渡越辐射一样, 衍射辐射干 涉强度分布就是单屏辐射分布和干涉因子 $\sin ^{2}\left(\frac{d}{2 L_{v}}\right)$ 的乘积, 其中 $d$ 是双屏间

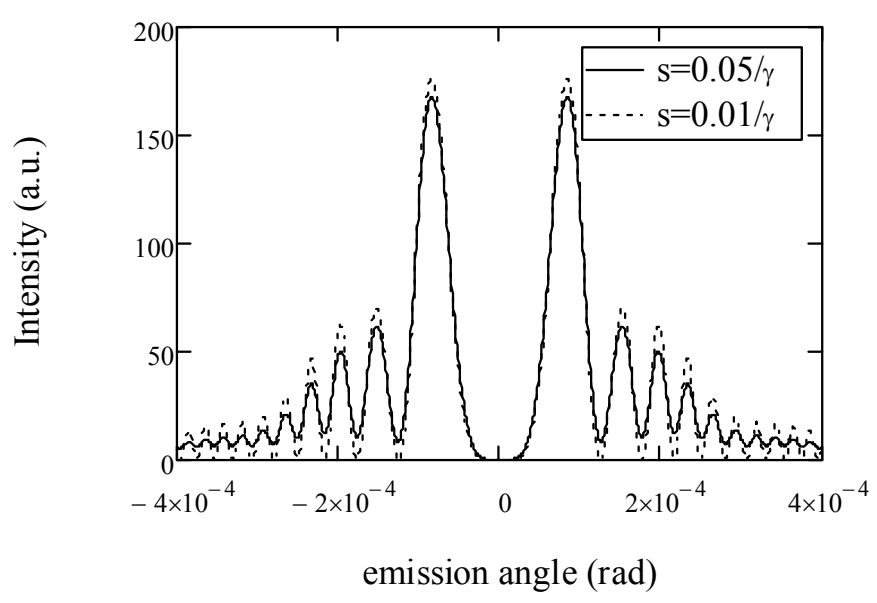

图 1.10 发散角对于衍射辐射干涉图案的影响

距, $L_{v}$ 是衍射辐射的相干长度。上面是取 $d=2 \gamma^{2} \lambda$, 不同散角下的干涉图案。 辐射分布的对比度主要受到相位项即干涉因子的影响。相比于上图, 干涉图 案对比度相比单屏辐射分布对于发散角更为敏感, 散角从 $0.01 / \gamma$ 到 $0.05 / \gamma$ 的变 
化所引起的对比度变化和单屏辐射分布中散角从 $0.1 / \gamma$ 到 $0.2 / \gamma$ 所引起的变化相 当。

\subsection{3 衍射辐射测量历史}

第一次衍射辐射实验[23]测量是 1995 年在 Tohoku University 完成的。实验中 使用的是 $150 \mathrm{MeV}$ 的短脉冲电子束, 衍射辐射是由开有圆孔的铝盘所产生的。他 们对毫米及亚毫米波范围的辐射进行了测量, 得到了渡越辐射和衍射辐射干涉的 角度分布。他们发现辐射强度和束流强度的平方成正比, 和理论上的非相干辐射 强度比, 观察到的辐射增强了 $1.5 \times 10^{8}$ 的量级, 该量级和电子束内电荷数目吻合。 根据测量的谱信息，通过计算得到了电子束团长度。下面是该装置使用的辐射屏:

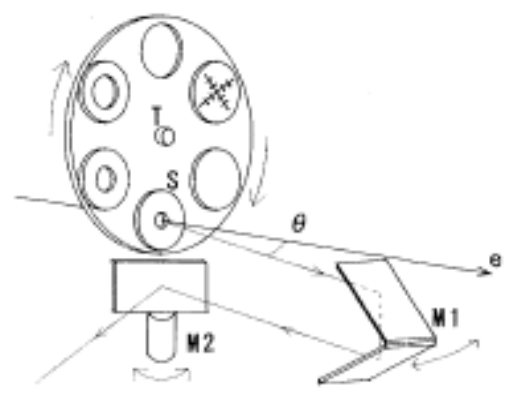

图 1.11 Tohoku University 使用的辐射屏

和实验测到的强度角分布和辐射谱:

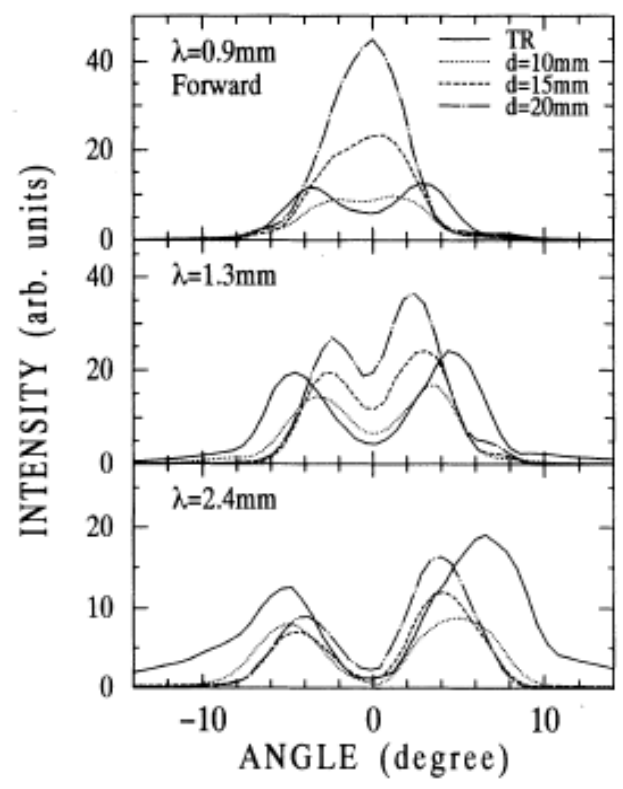

图 1.12 衍射辐射和渡越辐射角分布 


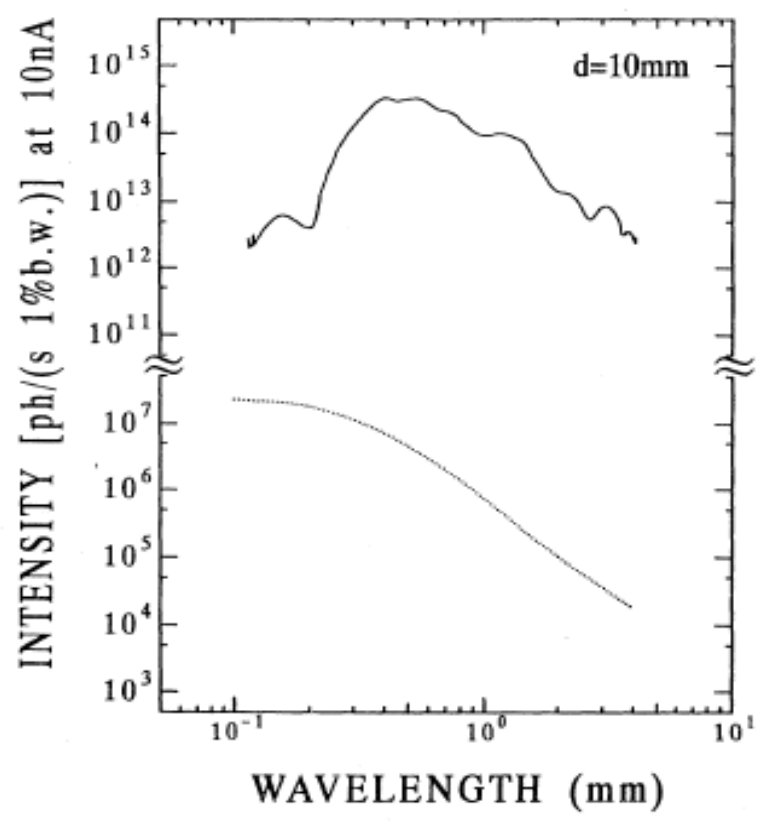

图 1.13 衍射辐射的谱分布

在 KEK-ATF 束流引出束线上进行的衍射辐射测量分为两个阶段：第一阶段 使用的是单边辐射屏，进行的衍射辐射观测实验[24]; 第二阶段使用了带狭缝的 辐射屏, 进行了束流尺寸的测量 $[25,26]$ 。两种情况都是辐射角度分布的测量。 下面是其基本束流参数:

表格 1.2 KEK-ATF 束流基本参数

\begin{tabular}{lc}
\hline \hline Energy & $1.28 \mathrm{GeV}(\gamma=2505)$ \\
Vertical emittance & $1.5 \times 10^{-11} \mathrm{~m} \mathrm{rad}$ \\
Horizontal emittance & $1.4 \times 10^{-9} \mathrm{~m}$ rad \\
Bunch population & $1.2 \times 10^{10}$ \\
Bunch length & $\sim 8 \mathrm{~mm}$ \\
\hline \hline
\end{tabular}

及实验装置设置:

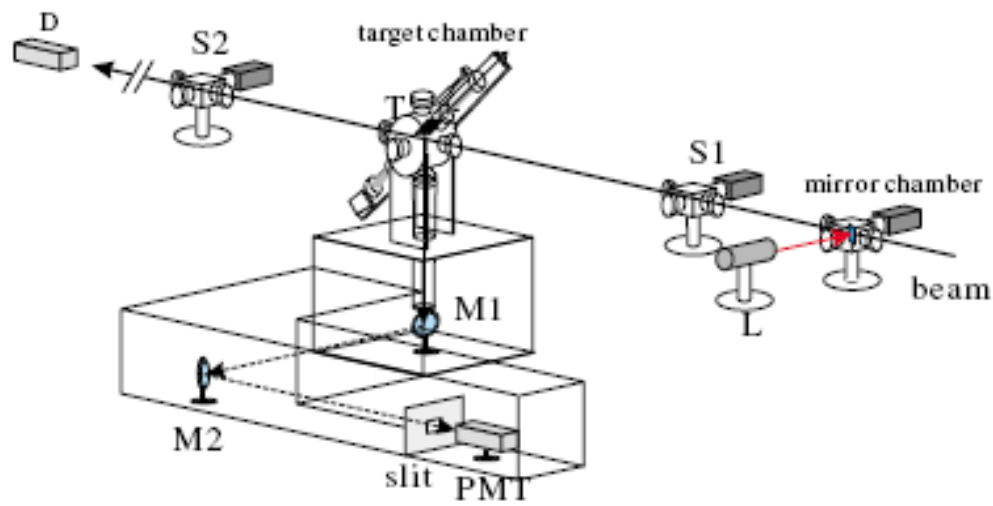

图 1.14 KEK-ATF 衍射辐射实验装置 
在第一阶段的测量中，其辐射屏的设置[27]如下:

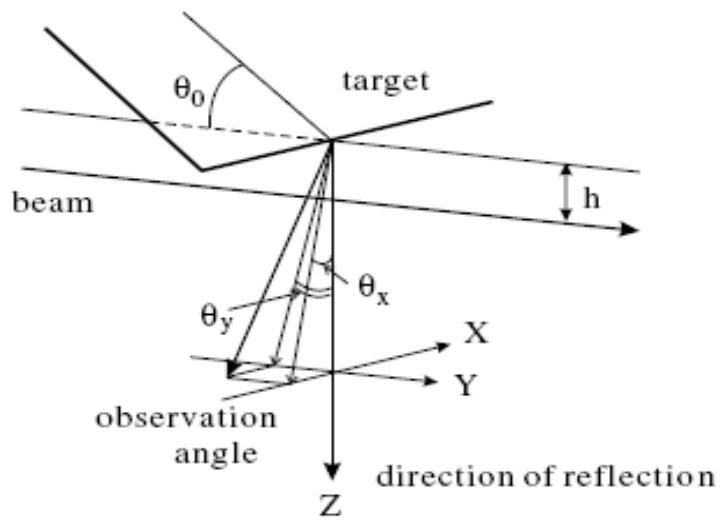

图 1.15 单边辐射屏设置示意图

在相对论电子的条件下, 与束流夹角为 $45^{\circ}$ 的辐射屏产生的辐射角分布可以表 示为:

$$
\frac{\partial^{2} W}{\partial \omega \partial \Omega}=\frac{\alpha}{4 \pi^{2}} \exp \left(-\frac{\omega}{\omega_{c}} \sqrt{1+\gamma^{2} \theta_{x}^{2}}\right) \times \frac{\gamma^{-2}+2 \theta_{x}^{2}}{\left(\gamma^{-2}+\theta_{x}^{2}\right)\left(\gamma^{-2}+\theta_{x}^{2}+\theta_{y}^{2}\right)}
$$

实验中先对 OTR 角分布进行了测量, 取得了预期的结果从而检验了光学测 量系统的可靠性。然后测量了可见光范围辐射强度与作用因子的依赖关系, 取得 了和理论[28]符合得很好的结果。同时也进行了适当作用因子下衍射辐射在水平 和坚直两个方向上的角分布。该一系列的实验测量第一次观察到了可见光范围非 相干衍射辐射, 取得了和理路计算符合的结果, 为将衍射辐射测量发展成为束流 尺寸的测量手段打下了坚实的基础。

第二次测量中辐射屏为带有 $0.26 \times 5 \mathrm{~mm}$ 丵孔的 $7 \times 9 \mathrm{~mm}$ 镀金硅片[29], 对于 可见光范围的测量, 该辐射屏可以被近似看作两个无限大的半平面。

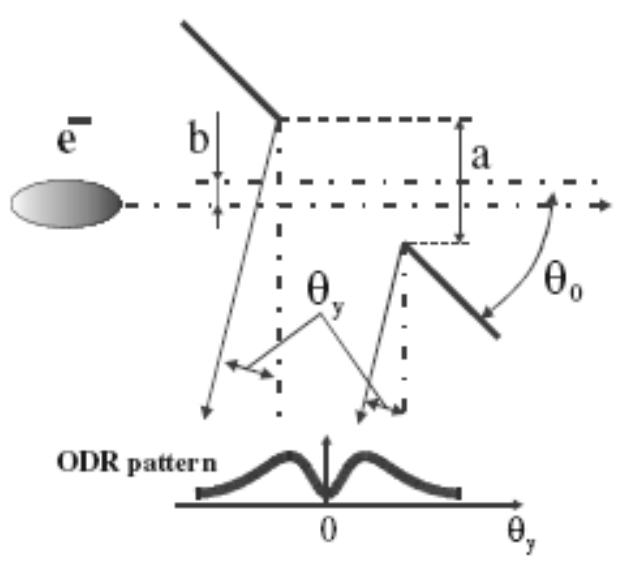

图 1.16 狭缝辐射屏示意图 
如前面所说, 束流穿过狭缝时在其两侧的金属靶上引起电荷分布的瞬时变化从而 产生衍射辐射, 两个半平面产生的后向传播辐射发生干涉产生干涉图案, 辐射坚 直极化成分角分布对于束流坚直束斑大小很敏感 [30]。坚直极化成分对于各参数 的依赖关系为:

$$
\begin{aligned}
\frac{\partial^{2} W_{y}^{\text {slit }}}{\partial \omega \partial \Omega}= & \frac{\alpha \gamma^{2}}{4 \pi^{2}} \frac{\exp \left(-z \sqrt{1+t_{x}^{2}}\right)}{1+t_{x}^{2}+t_{y}^{2}} \times\left\{\exp \left[\frac{2 z^{2} \sigma_{y}^{2}}{a^{2}}\left(1+t_{x}^{2}\right)\right]\right. \\
& \left.\times \cosh \left[\frac{2 z b}{a} \sqrt{1+t_{x}^{2}}\right]-\cos \left[z t_{y}+2 \psi\right]\right\}
\end{aligned}
$$

依据上面的理论公式, 坚直极化分量的分布和小的坚直束流尺寸有着更加显著的 相关关系。在实验中所作的测量是坚直极化分量的投影分布, 计算角分布中谷底 强度与峰值强度的比率。通过对比理论计算来得到束流尺寸。值得说明的是, 该 方法适用于发射度非常低的束流。在 KEK-ATF, 坚直发射度为 $1.5 \times 10^{-11} \mathrm{mrad,}$ 假设坚直 RMS 束斑大小是 $10 \mathrm{um}$, 那么坚直 RMS 发散角为 $1.5 \mathrm{urad}$, 这是束流尺 寸是坚直极化分布的决定因素。下面是测量到的 OTR 和 ODR 角分布[26]

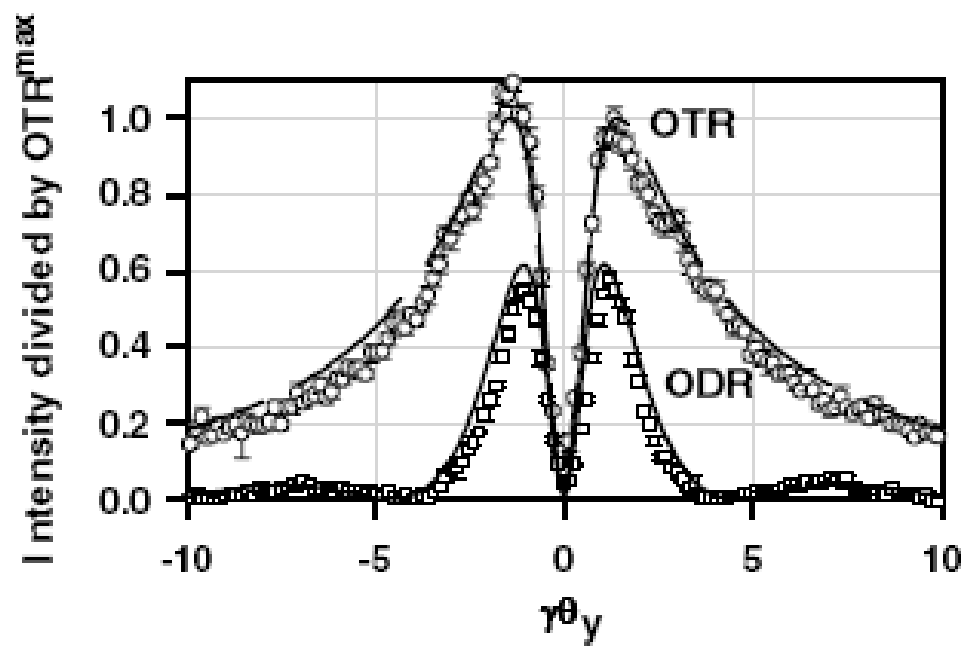

图 1.17 测量到的 ODR 和 OTR 辐射角分布

在上游 $0.7 \mathrm{~m}$ 和下游 $1.51 \mathrm{~m}$ 处两个坚琴测量装置的帮助下, 他们进行了 ODR 束斑测量和坚琴绝对束斑测量的比较。很明显, ODR 测量在束斑接近 10um 时 出现了较大的出入。当然, 这种差异可能来自于坚琴测量, 因为其精度受到丝线 直径的限制。 


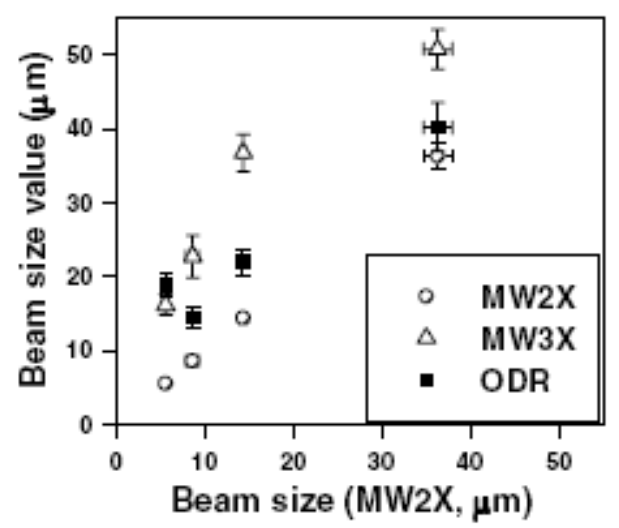

图 1.18 ODR 和坚琴束斑测量结果比较

另外, 束流位置也会影响辐射角度分布, 而且和束流尺寸的影响效果是一样 的。因此, 先将束流固定在狭缝中心来排除束流位置的影响是很关键的。上游使 用的带孔径的陶瓷盘很好地消除了同步辐射或边缘辐射的影响[31]。他们所进行 的角分布的测量是通过沿水平和坚直方向旋转反射镜 M1 来实现的, 因此进行一 次完整的测量角分布需要 10 分钟的时间。

R. B. Fiorito等人在BNL及NPS的能量分别为 $50 \mathrm{MeV}$ 和 $95 \mathrm{MeV}$ 的电子直线加速 器上成功进行了 ODR + OTR干涉测量[22], 并通过模拟计算得到了束流发散角。 衍射图案是由网耖（Micro mesh）产生的前向ODR和下游辐射屏上产生的后向 OTR发生干涉而产生的。通过对于网篮参数、网篮与下游OTR屏间距、观测波长 和滤波片的选择，干涉图案在本底的基础上清晰可见并受束流散角变化的影响。 下面是辐射产生元件的示意图:

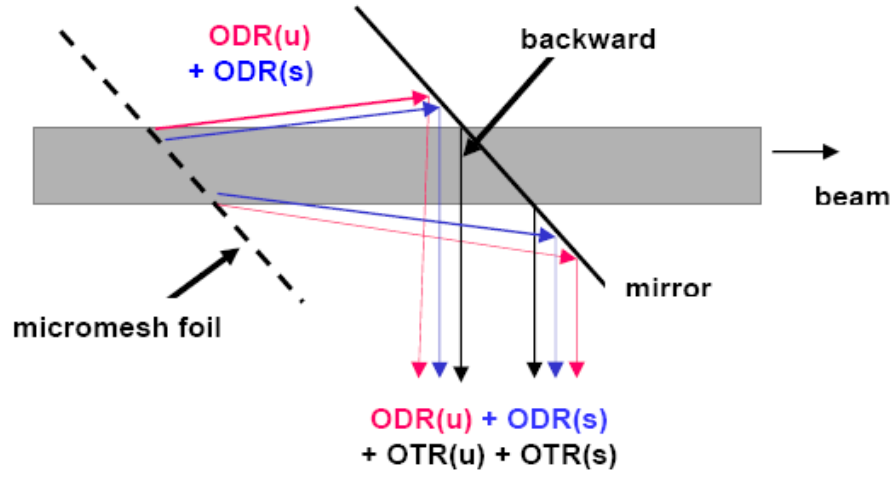

图 1.19 ODR+OTR 干涉仪示意图

他们使用了除了 ODR + OTR辐射外的另外两种手段来进行相同的测量：渡越辐射 相干（OTRI）和多屏测量。在两台机器上不同的发散角测量取得了和好的吻合。 
下面是实验装置示意图

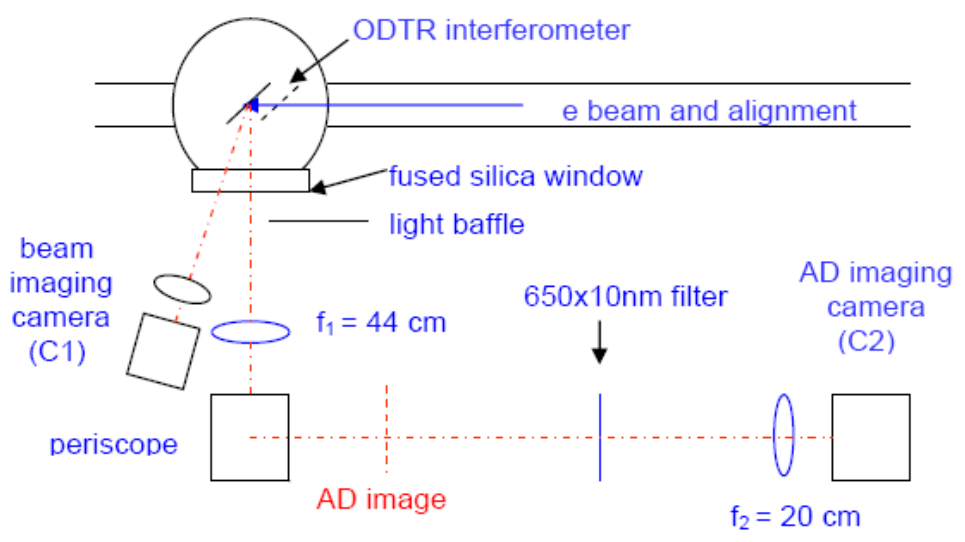

图 1.20 ODR+OTR 干涉实验装置示意图

位于上游的网篮为角分布的测量提供了很好的角度定标。在激光照射时, 因 为网笁的存在而会产生衍射现象, 衍射图案是常见的点阵分布, 如光学实验所演 示的那样, 每一点和中心点的夹角是可计算的, 因此, 通过 CCD 相机对该衍射 点阵分布的测量可以很精确地定标角度。

该装置中使用到的靶梯被步进电机所控制，其中四个元件都可以被定位于束 流中心, 它们分别是：定标格、用于准直的镀铝硅片、OTR 干涉仪及 ODR + OTR 干涉仪[32]。BNL 的 ATF 直线加速器的参数[33]如下： 1.5pps, 500-700pC/pulse; NPS 电子束的参数如下: $60 \mathrm{pps}$, 平均电流 0.1-0.8uA。归一化 RMS 发射度分别 为 1 及 $200 \mathrm{mmmrad}$; 能散分别为 $0.5 \%$ 和 $5 \%$; 在实验中所使用的束斑大小分 别为 100um 和 1000um。下面是在 NPS 机器上 60 秒时间内测量的干涉图案分 布以及多参数模拟的结果:
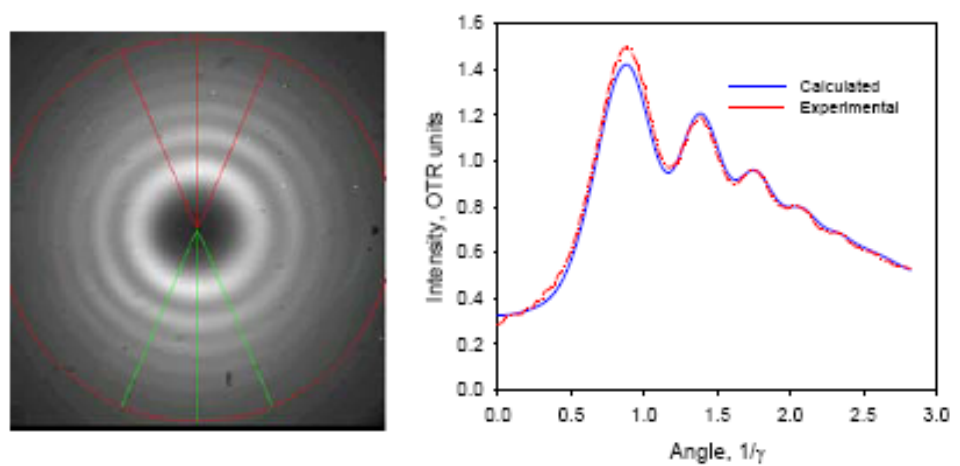

图 1.21 ODR+OTR 干涉图案及曲线拟合

OTR 干涉测量（此处并未显示）中给出的发散角是 $0.58 \mathrm{mrad}$, 而 ODR+OTR 干 涉测量模拟得出的发散角是 $0.56 \mathrm{mrad}$ 。更窄的滤波器以及相对于 NPS 更低的平 
均电流强度导致在 BNL ATF 进行的 ODR + OTR 干涉测量和 OTR 干涉测量所需 要的时间更长, 分别为 480 和 360 秒。两个测量中多种测量方式得到的结果都出 现了很好的符合。

尽管 KEK 进行的束斑大小测量因为低发散角的原因取得了好的测量结果, 但并不能使用于一般的束流条件。而且, 需要使用旋转镜来扫描使得整个测量过 程相当冗长。A. H. Lumpkin 在 APS 上进行的单边衍射辐射的近场测量排除了发 散角的影响, 被证明可以用于束流位置及束斑大小的非拦截测量手段[34]。下面 是该实验装置的俯视图:

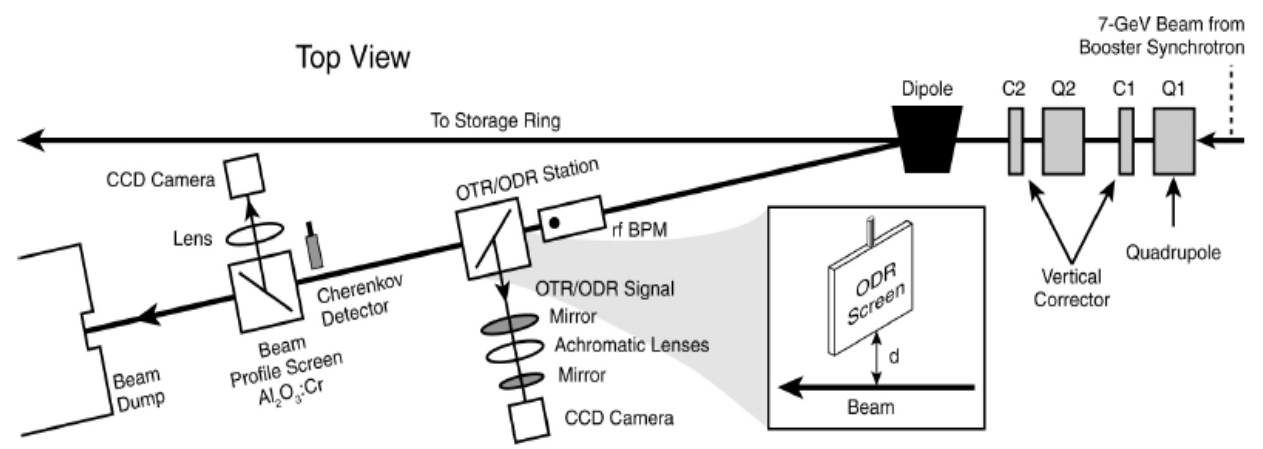

图 1.22 APS 衍射辐射实验示意图

在能量为 $7 \mathrm{GeV}$, 束团电荷量为 $0.4 \mathrm{nC}$ 的条件下进行了 OTR 测量; 在束团电荷量 为 $3.3 \mathrm{nC}$ 时进行了 $\mathrm{ODR}$ 的测量。下面是分别的测量结果:
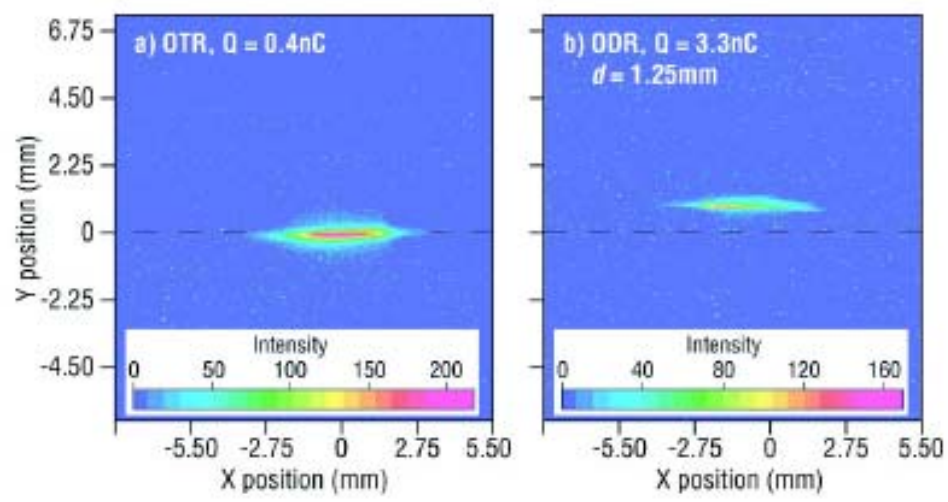

图 1.23 OTR 和 ODR 近场辐射测量

电子束经过单边辐射屏时, 引起辐射靶产生辐射。该辐射的分布沿水平方向 是近高斯分布，沿坚直方向往远离束流的方向则是近指数递减的分布[35]。实验 数据的后处理就是要找到水平高斯分布和束斑大小的依赖关系, 从而反推束斑大 小。下面展示的是 ODR 和 OTR 测量的直接比较以及 $\mathrm{Q}$ 铁扫描下的二者结果的 比值。 

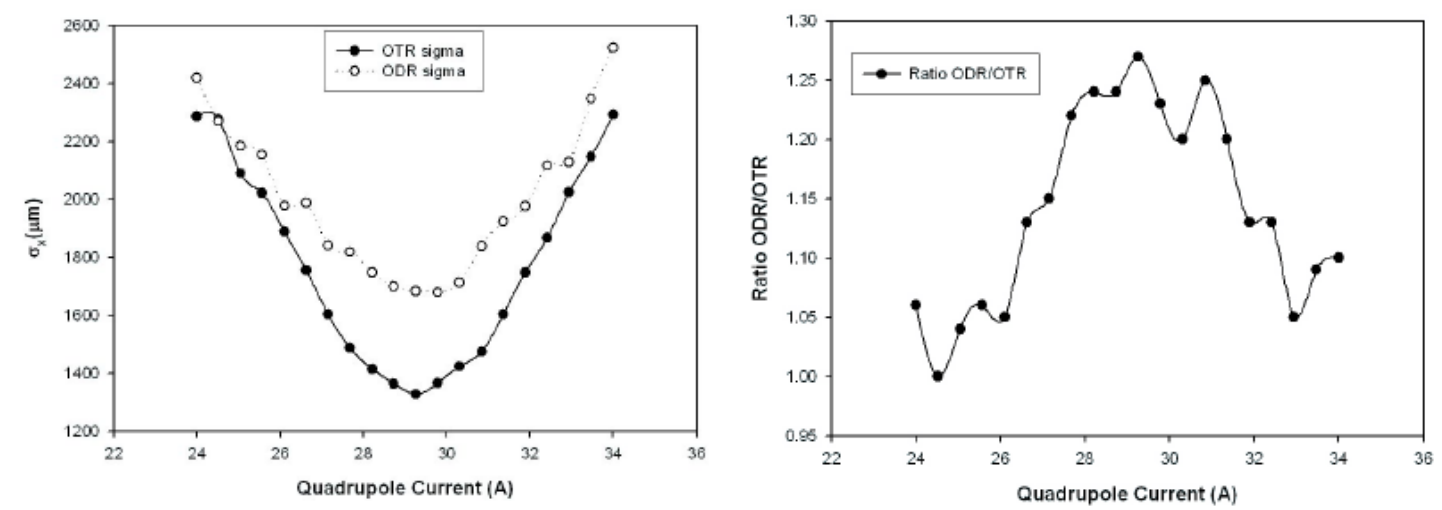

图 1.24 四极铁扫描下 ODR 和 OTR 束斑测量比较

OTR 测量作为一种成熟的束斑测量技术，其测量结果被当做是实际束斑大小。 两种辐射分布宽度 $(\sigma)$ 存在很明显的关联: ODR 测量结果随 OTR 测量结果出 现同步的上升和下降变化。然而, 二者的比例在一定范围内任意波动, 并无规律 可循。为了将该方法发展成在线测量手段，必需提前在调机模式下对两种方法进 行足够多的比较测量, 得到在不同束斑下的比值。同时, ODR 作为束流位置测 量的可能性也在试验中得到了证明。

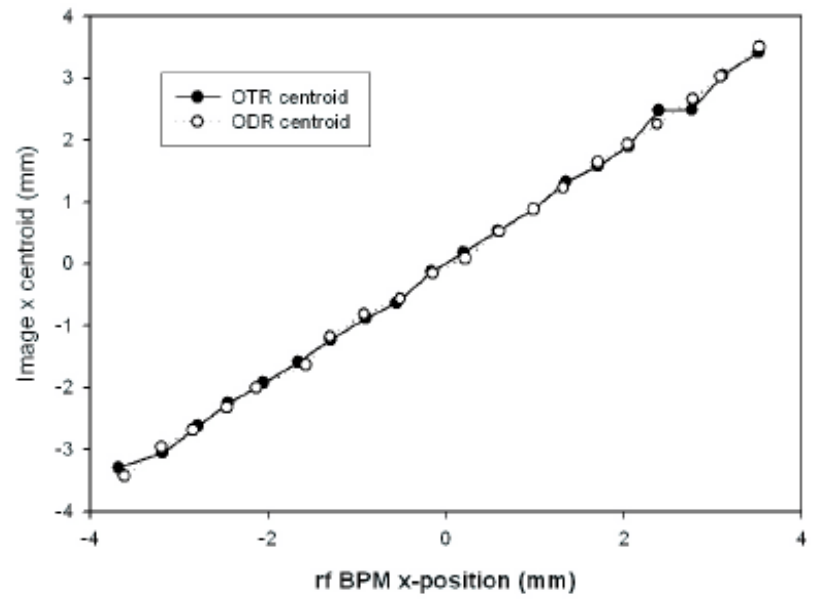

图 1.25 比较 ODR 和 OTR 位置测量

图中可见 OTR 和 ODR 的中心重合得很好。该束流位置测量的精度在 $200 \mathrm{um}$, 在 更高电流强度和更小束斑的机器中，更好的测量精度应该很容易实现。

M. Castellano等人在DESY也进行了一系列的衍射辐射的测量实验。首先, 他 们做的是束团长度的测量[36]。产生衍射辐射的是狭缝辐射屏, 而测量的是 $\mathrm{THz}$ 范围的相干辐射。该辐射谱低频截至波长有束管尺寸确定, 高频截至频率则由狭 缝宽度和真空窗的吸收决定。 


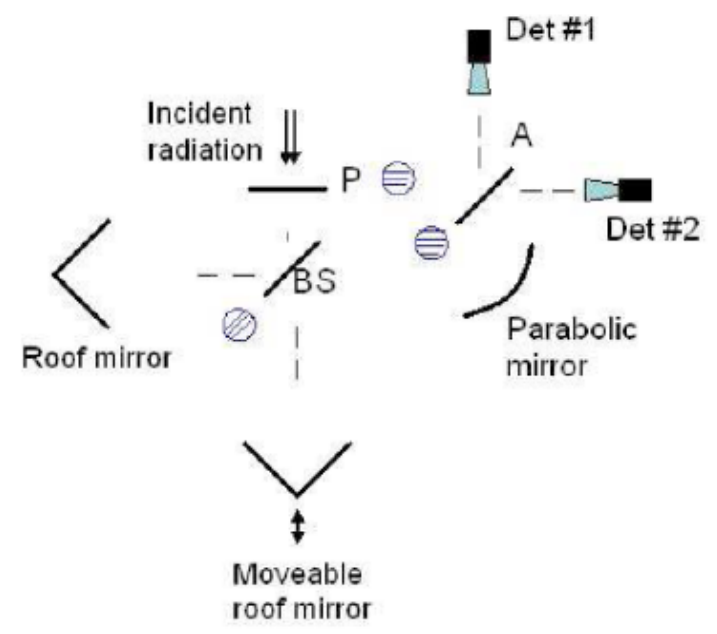

图 1.26 用于束团长度测量的 M-P 干涉仪示意图

THz 范围的辐射进入 M-P 干涉仪[37], 相互垂直的极化分量被分开、偏转然 后重新组合。M-P 干涉仪的优点在于对于电荷抖动不敏感。为了能从干涉图推导 的辐射谱中提取束团长度的信息, 需要对各个光学元件的频率传输函数进行仔细 的分析和对探测器进行认真的标定。

作为 FEL 的先驱, DESY 充分认识到 FEL 过程对于任何束流扰动都会相当 的敏感, 包括产生衍射辐射时有可能发生的尾场对束流的扰动。因此, 他们在 FEL 发光的同时进行了衍射辐射的测量, 并未发现发光过程受到干扰。这说明, 衍射辐射是一个真正意义上的非拦截测量工具[38]。

在此之后, 他们还进行了束斑大小的测量, 基于的原理和 KEK-ATF 一样: 测量狭缝产生的辐射角度分布并分析拟合处束斑大小。在他们的测量中, 其他辐 射本底是一个很大的限制因素。他们所用的办法是利用软件来计算并扣除同步辐 射本底。解决本底问题后, 他们取得了测量和理论计算很好的辐射结果:

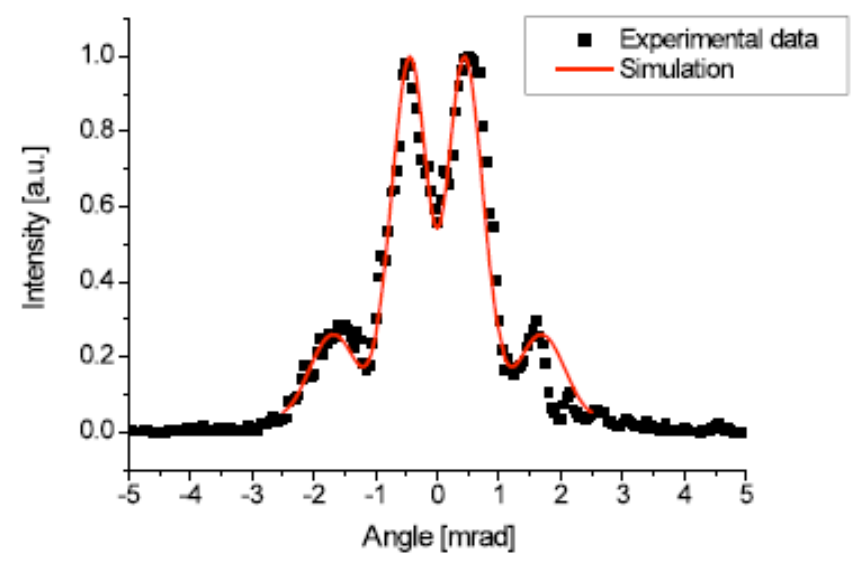

图 1.27 狭缝衍射辐射角分布测量及模拟对比 
为了从根本上解决本底问题, 他们安装了带狭缝的不锈钢片在 ODR 屏的上 游。然而, $1 \mathrm{GeV}$ 的电子束流同样引起了 $1 \mathrm{~mm}$ 狭缝的钢片上衍射辐射的产生, 其中的前向衍射辐射和原先 ODR 屏产生的后向衍射辐射发生相干, 形成了 ODR 干涉仪。尽管参数并没有经过刻意优化, 他们还是得到了对比度挺高的干涉图案, 但是并不是像理论预计的对称图案。究其原因, 是 ODR 辐射屏的两块半平面并 不严格在同一平面上所引起的。

\section{3 CEBAF简介}

\section{CEBAF[39]（Continuous Electron Beam Accelerator Facility）是Thomas} Jefferson National Facility两台电子加速器（另一个是Jlab-FEL）之一。该机器的 建设和调试开始于上世纪 90 年代初，90年代中 CEBAF就开始同时为三个物理实 验室提供能量不一 (最高 $4 \mathrm{GeV}$ ) 的电子束流[40]。2000年, 随着射频超导加速技 术的发展最高能量被提升至 $6 \mathrm{GeV}$ 。

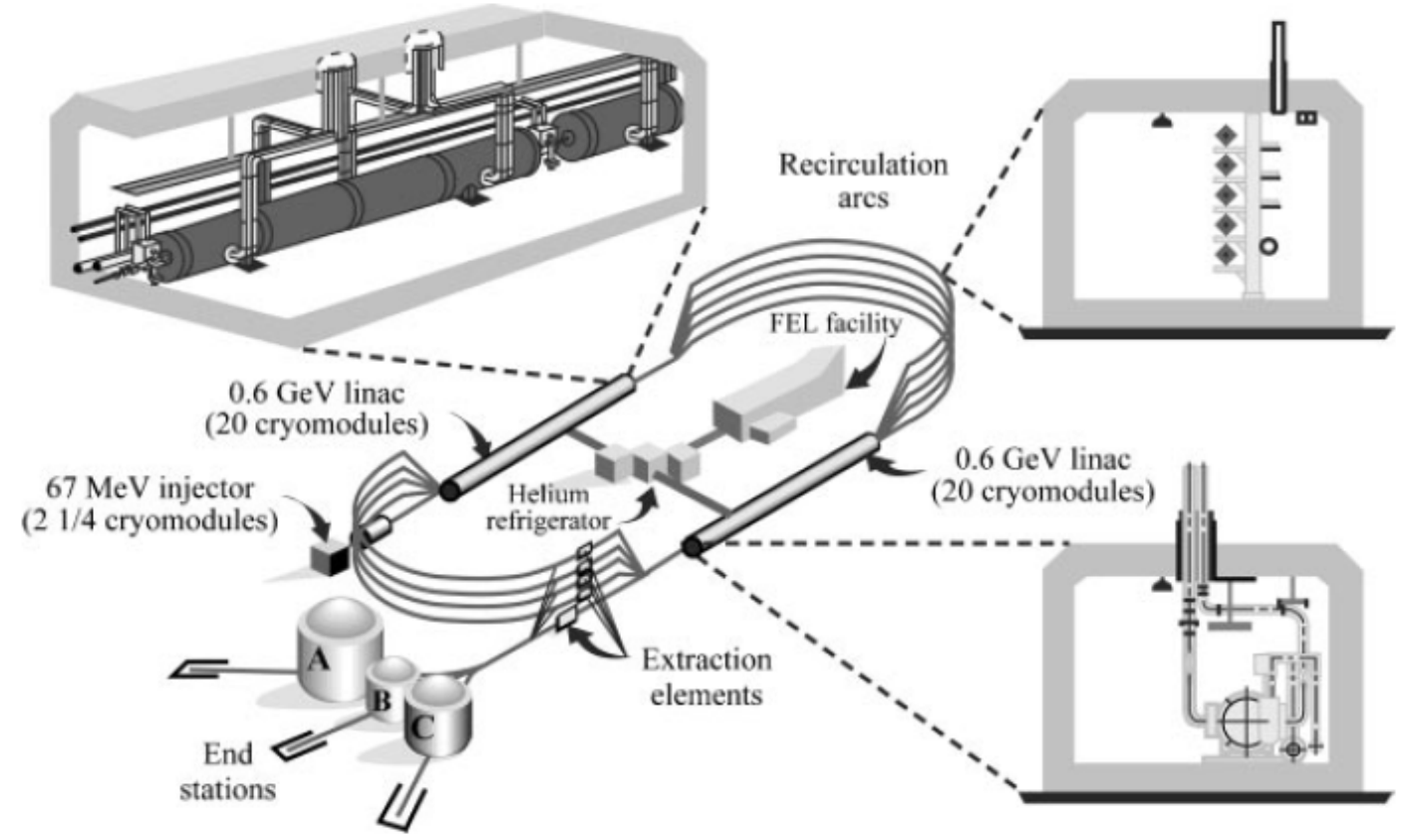

图 1.28 CEBAF 机器总览图

CEBAF 是一个可以 5 圈返航加速的射频超导直线电子加速器, 它同时为三 个终端实验站提供分别为 $200 \mathrm{uA} 、 \sim 100 \mathrm{nA} 、 \sim 200 \mathrm{uA}$ 的极化度高于 $75 \%$ 的电子 束流, 几何发射度小于 $10^{-9} \mathrm{mrad}$, 相对能散为几个 $10^{-5}$ 。现可运行的最低能量为 $0.6 \mathrm{GeV}$, 最高可达 $6 \mathrm{GeV}$ 。机器已于 2009 年开始升级工作, 并将于 2015 年试运 
行于 $12 \mathrm{GeV}$ 的能量。5 圈返航加速、三台光阴极光纤驱动激光和 $500 \mathrm{MHz}$ 的射 频腔分离器的结合使得 CEBAF 可以同时输运不同参数的三条束流, 不同实验站 的电流强度相差 6 个量级。在 CEBAF 上最重要的创新是射频超导技术和多圈返 航加速的实现。在 LEP II 运行之前, CEBAF 是世界上最大的应用射频超导技术 的加速器。并且射频超导技术的应用方便了未来的能量升级, 为物理研究保留着 发展的空间。返航加速技术的成功实现在很大程度上减小了所需要的射频超导费 用, 弯转段的设计也为能量升级保留了空间。下面是 CEBAF 参数[39]一览表:

表格 1.3 CEBAF 束流参数总览表

\begin{tabular}{ll}
\hline Energy & $5.71^{*} \mathrm{GeV}$ \\
Average current (Halls A and C) & $1-150 \mu \mathrm{A}$ \\
Average current (Hall B) & $1-100 \mathrm{nA}$ \\
Bunch charge & $<0.3 \mathrm{pC}$ \\
Repetition rate & $499 \mathrm{MHz} / \mathrm{hall}$ \\
Beam polarization & $>75 \%$ \\
Beam size (rms transverse) & $\sim 80 \mu \mathrm{m}$ \\
Bunch length (rms) & $300 \mathrm{fs}, 90 \mu \mathrm{m}$ \\
Energy spread & $2.5 \times 10^{-5}$ \\
Beam power & $<1 \mathrm{MW}$ \\
Beam loss & $<1 \mu \mathrm{A}$ \\
Number of passes & 5 \\
Number of accelerating cavities & 338 \\
Fundamental mode frequency & $1497 \mathrm{MHz}$ \\
Accelerating cavity effective length & $0.5 \mathrm{~m}$ \\
Cells/cavity & 5 \\
Average $Q_{0}$ & $4.0 \times 10^{9}$ \\
Implemented $Q_{\text {ext }}$ & $5.6 \times 10^{6}$ \\
Cavity impedance $(r / Q$ ) & $980 \Omega$ \\
Average cavity accelerating gradient & $7.5 \mathrm{MV} / \mathrm{m}$ \\
RF power & $<3.5 \mathrm{~kW} / \mathrm{cavity}$ \\
Amplitude control & $1.00 \times 10^{-4} \mathrm{rms}$ \\
Phase control & $0.1^{\circ} \mathrm{rms}$ \\
Cavity operating temperature & $2.08 \mathrm{~K}$ \\
Heat load @ 2 K & $<9 \mathrm{~W} / \mathrm{cavity}$ \\
Liquifier 2 K cooling power & $5 \mathrm{~kW}$ \\
Liquifier operating power & $5 \mathrm{MW}$ \\
\hline & \\
\hline
\end{tabular}




\section{4 为什么CEBAF需要衍射辐射测量}

二零零七年, 在 CEBAF 机器上应用衍射辐射近场辐射测量束斑大小被提出 [41]。该提议主要基于下面三方面的考虑:

首先, CEBAF 机器上束流尺寸正是所感兴趣的范围。近似对称分布的 CEBAF 束流 RMS 束斑（ $80 \mathrm{um})$ 比 APS 束斑（ 1300um）小十几倍, 该束斑还有可以 利用光学元件进行压缩的空间。更重要的是, APS 升级[42]和 ILC 的设计 [43, 44] 束斑大小和 CEBAF 束斑大小在相近的范围。因此, CEBAF 上衍射辐射的测量 能很好地检验该测量手段在这些筹建的大型加速器上应用的可行性。

另外, CEBAF 上的连续束流可以产生更强的辐射进而可以进行更高精度的 测量。物理实验站上束流的重复率是 $499 \mathrm{MHz}$, 平均电流强度是 $100 \mathrm{uA}$, 因此电 子束团的电荷量是 $0.2 \mathrm{pC}$ 。一般的交织式扫描 $\mathrm{CCD}$ 相机的重复率是 $50 \mathrm{~Hz}$, 因此 图像积分时间为 $20 \mathrm{~ms}$ 。在积分时间内经过辐射屏的束团数目是 $10^{7}$, 积分电荷 是 $2000 \mathrm{nC}$ 。相当于 $\mathrm{APS}$ 实验中积分电荷 $(\sim 3 \mathrm{nC})$ 的 667 倍！即使在调机模式 下, 束流的占空比为 $1.5 \%$, 积分电荷也是 APS 积分电荷的 10 倍。因此, 可以 预期在合理的参数选择下, CEBAF 束流的衍射辐躬强度将会提高几个量级, 从 而有助于测量精度的提高, 并且使得在线测量实际可行。

最后, CEBAF 机器需要非拦截式束斑测量办法。现有的束斑测量办法有坚 琴测量 (Harps measurement)、荧光靶测量、OTR 测量和同步辐射光测量。前二 者都是拦截式测量; OTR 测量只能工作在调机模式下且对束流的扰动不能被物 理实验所接受, 因此也不理想; 而同步辐射光测量只能使用在弯转段。当物理实 验需要知道束斑大小, 像 ODR 这样的非拦截手段是亟需的。如研究束斑大小和 束流螺旋性的关系 [45], 束流螺旋状态以 $30 \mathrm{~Hz}$ 的频率变化, 除了 ODR 测量上面 所提到的常规测量方法显然是不能完成任务的。

\section{5 论文的工作和创新点}

本论文的工作分为两个部分, 一是利用衍射辐射进行的束斑测量, 二是用于 反康普顿电子极化仪的激光系统。

束斑测量的地点选在了 CEBAF Hall A 的束线上, 通过对于光学元件的调节, 
该段的束斑大小可以在几十至几百微米间调节。在该范围的成功测量将会很好地 证明衍射辐射测量的普适性。在该辐射测量中使用到的是近场测量模式, 即测量 辐射强度在辐射屏的分布情况。通过改变 $\mathrm{Q}$ 铁强度, 分别测量了不同束斑下总 辐射强度的分布、水平极化分量分布和坚直极化分量分布; 相同的情况下, 也进 行了渡越辐射测量和坚琴测量, 并对不同结果进行了比较; 改变驱动激光强度, 对不同电流强度束流进行了衍射辐射测量, 在强流下使用窄带滤波片进行了近似 无色差测量; 也测量了不同位置束流的衍射辐射分布图案; 还研究了极化波片对 渡越辐射测量的影响。与其他测量方式及现有衍射辐射测量相比较, 这部分论文 工作的价值主要在于两点: 一方面该测量办法有独特的优越性, 填补了几十到几 百微米范围束斑非拦截测量的空白; 另一方面, 我们得到的衍射辐射分布大小与 实际束斑大小的简单正比关系为该测量方法的普及应用奠定了坚实的基础。

论文中用于反康普顿电子极化仪的激光系统的工作是该项目第一阶段的工 作, 至今为止所建立的低功率系统包括完整的激光源、放大器和倍频元件, 第二 阶段的工作将会集中在激光功率的提高上。该激光系统主要由增益开关二极管激 光源、光纤放大器和 LBO 倍频器所组成。该项工作的意义在于利用增益开关光 纤耦合二极管技术, 保证了高品质单模激光输出; 以及演示了全光纤脉冲选择技 术, 拓展了该激光系统的应用范围; 还有开发了一种全新的脉冲生成技术, 提升 了脉冲序列的相干性。

\section{6 论文的结构}

论文第一到第五章为第一部分, 讲述的是利用衍射辐射对 CEBAF 机器 Hall A 束线上束斑大小的测量。

第一章主要概述和比较了现有的束斑测量办法, 阐述了在几到几百微米范围 内发展廉价、快速非拦截测量方法的必要性; 介绍了衍射辐射的基本概念、测量 方法、发展历史及应用现状; 然后给出了 CEBAF 机器的总体介绍及在 Hall A 束 线上进行衍射辐射测量的缘由。

第二章主要着重于衍射辐射的基本理论。介绍了相对论带电粒子的电磁场分 布, 及其被物体散射的近似模型一虚拟光子模型; 还有虚拟光子模型在单边衍射 辐射下的应用; 以及光辐射测量的两种实验设置一近场测量和远场测量; 最后探 
讨了光辐射测量中的测量精度问题。

第三章主要是衍射辐射测量实验装置的介绍。首先给出了装置的总体图和介 绍, 然后分别介绍了准直激光、扩束器、反射镜、OTR 及 ODR 辐射屏、光学成 像系统及滤波片和极化波片; 还介绍了系统准直的过程以及坚琴测量装置。

第四章的内容是对测量过程的介绍及结果的呈现。首先介绍了测量系统的空 间定标，然后分别给出了坚琴测量、OTR 测量和 ODR 测量的结果。

第五章是对测量结果的分析处理。首先介绍了处理软件及处理方法; 然后解 释了衍射辐射中水平极化非对称性及解决办法, 解释了辐射干扰的来源及给出了 消除其影响的提案; 分析了衍射辐射对于束流电流强度和测量频带的依赖关系; 最后总结了衍射辐射分布大小与实际束斑大小的关系。

论文第六和第七章为第二部分, 讲述的是用于反康普顿电子极化仪的激光系 统。

第六章主要介绍反康普顿散射、加速器应用对于激光的基本要求、Hall C 反 康普顿电子极化仪及其对激光参数的要求。

第七章给出了低功率激光系统的总体介绍。首先是激光源的选择, 增益开关 二极管激光源的介绍及相关的参数测量, 还有验证全光纤脉冲选择技术的过程。 然后是光纤放大器, 及激光传输（品质）测量; 还有倍频晶体的选择, 以及倍频 实验结果和模拟结果; 最后给出了用于功率升级的放大器设计。 


\section{第二章 衍射辐射原理}

本章的目的是研究衍射辐射原理, 并以此出发讨论理论上衍射辐射分布情 况, 以及能分析得到的束流参数。在此基础上确定辐射测量的重要参数和基本思 路。

衍射辐射是带电粒子电磁场与非均匀介质环境相互作用的结果, 也可以被理 解为带电粒子在非均匀介质环境中所产生的尾场效应。因此, 对于衍射辐射的分 析包括对带电粒子电磁场的分析及其与介质相互作用原理分析。

本章从运动电子的电磁场出发, 介绍相对论电子的电磁场特性, 以及其与电 磁波的相似性, 然后介绍用于相对论电子的虚拟光子模型, 还有其在衍射辐射分 析上的应用。

\section{1 运动电子的电磁场}

假设一个电子以速度 $v$ 匀速运动, 来计算其在实验室坐标系 $(\mathrm{K})$ 中一静止 点 $(\mathrm{P})$ 处所产生的电磁场。

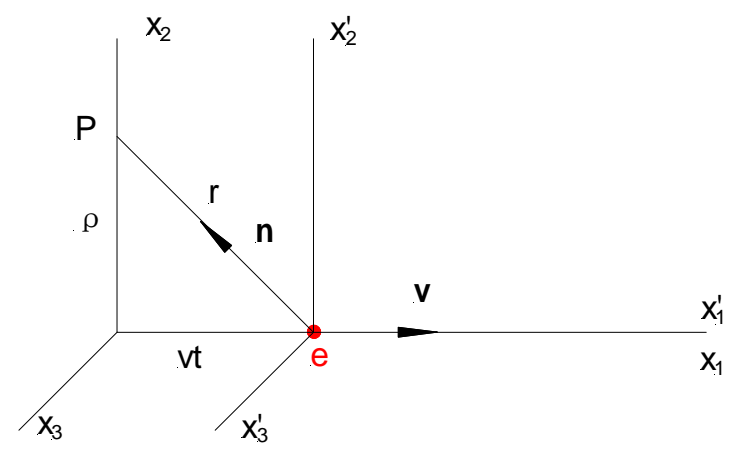

图 2.1 运动电荷产生的电磁场

运动坐标系 $\left(\mathrm{K}^{\prime}\right)$ 以速度 $v$ 向坐标轴 $\mathrm{x}_{1}\left(\mathrm{x}_{1}{ }^{\prime}\right)$ 的方向运动, 在该坐标系内电子 静止不动。观察者位于 $\mathrm{P}$ 点并在实验室坐标系 $(\mathrm{K})$ 内静止。在时刻 $\mathrm{t}=\mathrm{t}^{\prime}=0$, 电 子和观察者位于同一横截面内并且距离最近。任意时刻 $\mathrm{t}^{\prime}$, 点 $\mathrm{P}$ 在坐标系 $\mathrm{K}$ ' 内 的位置是 $(-v \mathrm{t}, \rho, 0)$ 。那么在运动坐标系中来看 $\mathrm{P}$ 点处在时刻 $\mathrm{t}^{\prime}$ 的电磁场是 [46]: 


$$
\begin{aligned}
& E_{1}^{\prime}=-\frac{1}{4 \pi \varepsilon_{0}} \frac{e \gamma v t}{\left(\rho^{2}+\gamma^{2} v^{2} t^{2}\right)^{3 / 2}} \\
& E_{2}^{\prime}=\frac{1}{4 \pi \varepsilon_{0}} \frac{e \rho}{\left(\rho^{2}+\gamma^{2} v^{2} t^{2}\right)^{3 / 2}} \\
& E_{3}^{\prime}=0 \\
& B_{1}^{\prime}=0 \\
& B_{2}^{\prime}=0 \\
& B_{3}^{\prime}=0
\end{aligned}
$$

在这里用到相对论能量因子 $\gamma$ 及相对速度 $\beta: \gamma=\frac{1}{\sqrt{1-\beta^{2}}}, \beta=\frac{v}{c}$ 。根据爱因斯 坦的狭义相对论 [47], 在运动坐标系与静止坐标系的相对速度为 $v$ 时, 两个坐标 系内的电磁场满足下面的洛仑兹(Lorentz)转换定律:

$$
\begin{aligned}
& E_{1}^{\prime}=E_{1} \\
& E_{2}^{\prime}=\gamma\left(E_{2}-v B_{3}\right) \\
& E_{3}^{\prime}=\gamma\left(E_{3}+v B_{2}\right) \\
& B_{1}^{\prime}=B_{1} \\
& B_{2}^{\prime}=\gamma\left(B_{2}+v E_{3}\right) \\
& B_{3}^{\prime}=\gamma\left(B_{3}-v E_{2}\right)
\end{aligned}
$$

通过以上的洛仑兹转换, 位于 $\mathrm{K}$ 坐标系内的 $\mathrm{P}$ 点的电磁场是:

$$
\begin{aligned}
& E_{1}=-\frac{1}{4 \pi \varepsilon_{0}} \frac{e \gamma v t}{\left(\rho^{2}+\gamma^{2} v^{2} t^{2}\right)^{3 / 2}} \\
& E_{2}=\frac{1}{4 \pi \varepsilon_{0}} \frac{\gamma e \rho}{\left(\rho^{2}+\gamma^{2} v^{2} t^{2}\right)^{3 / 2}} \\
& B_{3}=\frac{1}{c} \beta E_{2}
\end{aligned}
$$

其它各项均为 0 。

\section{2 虚拟光子模型}

虚拟光子方法 (Pseudo-photon model) 最早由 E. Fermi 提出并得到 E. Williams 后面进一步的发展。它是类似于微扰理论计算的近似计算办法, 优点在于它有比 微扰理论中繁复的计算更高的效率。它主要被利用于涉及到相对论电荷的电磁相 互作用的物理过程，如轫致辐射、电子对的产生等[14]。该方法的主要物理思想 
是基于高速运动带电粒子的场和电磁波的相似性[14]。

从(2.3)可以看出, 当速度 $v$ 接近光速 $c$ 时, 相对于电场的横向分量(E2)来讲电 场的纵向分量(E1)很小。这时, 运动电子的电场分布呈 “薄饼” (pancake)形状, 沿横向延伸但被局限于很窄的纵向空间内。同时磁场分量 (B3)趋近于横向电场 (E2)的 $1 / c$ 倍。对于在真空或介质中传播的电磁波, 电场和磁场相互垂直而且二 者垂直于电磁波能量传播的方向。因此, 高速运动的带电粒子所产生的电磁场除 了有一个很小的纵向电场分量外与电磁波的分布具有高度的相似性。对于超高相 对论粒子, 其产生的场具有和电磁波一样的属性。电磁波由不同频率的波(光子) 所组成, 那么, 带电粒子就可以被等效为一系列不同频率的光子的集合。虚拟光 子方法就是用电磁波序列代替运动粒子的场, 在该近似的基础上, 相对论电子的 辐射过程就可以被处理为虚拟光子的散射。

考虑 $\beta \sim 1$ 的情况, 这时电场 $\mathrm{E} 2$ 和磁场 B3 的组合正好等效于沿纵向传播的 线性极化脉冲, 表示为 $\mathrm{P} 1$ 。电场 $\mathrm{E} 1$ 并没有相应的磁场组合成类似的脉冲。在所 考虑的辐射问题中, 电场才是真正的驱动场, 所以为了问题的方便添加或删减磁 场并不影响最终的结果。这样, 电场 $\mathrm{E} 1$ 在一个假设磁场的组合下也能等效于一 个沿 $\mathrm{x}_{2}$ 方向的线性脉冲 $\mathrm{P} 2$ 。在下面的表达中, 统一使用坐标 $(\mathrm{x}, \mathrm{y}, \mathrm{z})$ 来代替 $\left(\mathrm{x}_{2}, \mathrm{x}_{3}, \mathrm{x}_{1}\right)$ 。那么, 纵向匀速运动的电子产生的电场也可以用以下表达式表示:

$$
E_{x, y}(\omega)=-\frac{i e}{2 \pi^{2} v} \exp (i(\omega / v) z) \int \frac{k_{x, y} \exp \left(i\left(k_{x} x+k_{y} y\right)\right)}{k_{x}^{2}+k_{y}^{2}+\alpha^{2}} d k_{x} d k_{y}
$$

或者

$$
\begin{gathered}
E_{x, y}=\sqrt{\frac{2}{\pi}} \frac{e \omega}{\gamma v^{2}} \exp [i(\omega / v) z] \frac{x, y}{\sqrt{x^{2}+y^{2}}} K_{1}\left(\alpha \sqrt{x^{2}+y^{2}}\right) \\
E_{z}=-i \sqrt{\frac{2}{\pi}} \frac{e \omega}{\gamma^{2} v^{2}} \exp [i(\omega / v) z] K_{0}\left(\alpha \sqrt{x^{2}+y^{2}}\right)
\end{gathered}
$$

其中, $\alpha=\frac{\omega}{\gamma v}, \omega$ 是电磁波频率, $\gamma$ 是洛伦兹因子, $v$ 是电子运动速度, $K_{0}$, $K_{1}$ 分别是零阶和一阶第二类修正贝塞尔函数(Modified Bessel function)。(2. 5) 实 际上是匀速运动电子产生的横向电场的傅里叶变换(Fourier transform)。在虚拟光 子概念里, 它们是频率为 $\omega$ 的虚拟光子的横向电场的两个分量。 
如果采用圆柱坐标系, 并且和笛卡尔坐标系使用相同的 $\mathrm{z}$ 轴, 以上的两个表达式 还可以写成下面的形式:

$$
\begin{gathered}
E_{\rho}(\omega)=\sqrt{\frac{2}{\pi}} \frac{e \omega}{\gamma \nu^{2}} \exp [i(\omega / v) z] K_{1}(\alpha \rho) \\
E_{z}(\omega)=-i \sqrt{\frac{2}{\pi}} \frac{e \omega}{\gamma^{2} v^{2}} \exp [i(\omega / v) z] K_{0}(\alpha \rho)
\end{gathered}
$$

其中, $\phi_{0}$ 是方位角, $\rho$ 是电子到的 $\mathrm{z}$ 轴的距离。

参考[46], 可以知道等效脉冲 P1 的频谱, 即单位面积单位频率间隔的能量可 以表示为

$$
\frac{d I_{1}}{d \omega}(\omega, b)=\frac{c}{2 \pi}\left|E_{\rho}(\omega)\right|^{2}
$$

同样, 脉冲 P2 的频谱为

$$
\frac{d I_{2}}{d \omega}(\omega, b)=\frac{c}{2 \pi}\left|E_{z}(\omega)\right|^{2}
$$

将(2.7)、(2.8)分别代入(2.9), (2.10), 可以得到:

$$
\begin{gathered}
\frac{d I_{1}}{d \omega}(\omega, b)=\frac{1}{\pi^{2}} \frac{q^{2}}{c}\left(\frac{c}{v}\right)^{2} \frac{1}{b^{2}}\left(\frac{\omega b}{\gamma v}\right)^{2} K_{1}^{2}\left(\frac{\omega b}{\gamma v}\right) \\
\frac{d I_{2}}{d \omega}(\omega, b)=\frac{1}{\pi^{2}} \frac{q^{2}}{c}\left(\frac{c}{v}\right)^{2} \frac{1}{b^{2}} \frac{1}{\gamma^{2}}\left(\frac{\omega b}{\gamma v}\right)^{2} K_{0}^{2}\left(\frac{\omega b}{\gamma v}\right)
\end{gathered}
$$

注意到脉冲 P2 表达式中有一个 $\frac{1}{\gamma^{2}}$ 因子, 所以在高相对论的情况下来自 P2 的作 用可以被忽略。在考虑带电粒子束的场和物质相互作用问题时, 需要将(2.11)沿 作用截面作积分，来计算单位频率间隔内的辐射能量。

\section{3 近场测量及远场测量}

在本论文中, 研究兴趣主要在于辐射的两方面特性: 一是辐射的强度分布, 另一个是辐射角度分布。对于这两方面特性的研究是通过采用不同的辐射探测系 统设置来实现的, 常见的可见光范围辐射测量则是依靠不同的光学成像系统来完 成。这两种不同的测量方式分别被称之为近场测量和远场测量 


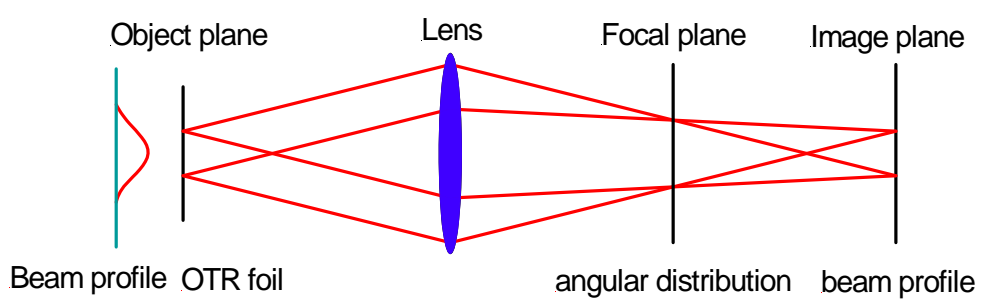

图 2.2 近场测量和远场测量示意图

图中以 OTR 辐射为例, 给出了用于近场测量和远场测量的简化成像系统。透镜 代表成像系统的光学元件, 如果观察者位于系统的焦平面位置, 其接收到的是辐 射强度随着角度的分布, 被称为远场测量; 如果观察者位于像平面的位置, 它看 到的是辐射强度在辐射屏上随着位置的分布(辐躬强度分布与束流电荷截面分布 成正比), 被称为近场分布[48]。

\section{4 单边虚拟光子模型}

在 Hall A ODR 装置中, 衍射辐射屏是长方形的镀铝硅片, 电子束径迹位于 辐射屏底边下方, 衍射辐射强度主要来自于屏底边, 这种情况下的辐射被称之为 单边衍射辐射[49]。下面给出了简化图, 红色小栯圆代表的高斯分布电子束沿垂 直纸面的方向向纸内运动, 想象电子束周围有一电磁场 “薄饼” 跟随其运动。基 于虚拟光子模型，该电磁场可以被看作虚拟光子集合，当电子束运动到屏的下方 时, 虚拟光子碰撞到金属屏上并被散射。假设金属屏为均匀介质，在屏上某一点 散射强度应正比于电子束在该处产生电场强度的平方, 而屏上某一点的电场强度 的平方是电子束内任意电子在该处电场强度平方对于电子束分布的卷积。根据公 式(2.11), 单边衍射辐射微分强度分布可以表示为,

$$
\frac{d I}{d \omega}(u, \omega)=\frac{1}{\pi^{2}} \frac{q^{2}}{c} \alpha^{2} N \frac{1}{\sqrt{2 \pi \sigma_{x}^{2}}} \frac{1}{\sqrt{2 \pi \sigma_{y}^{2}}} \iint d x d y K_{1}^{2}(\alpha b) \exp -\left[x^{2} / 2 \sigma_{x}^{2}\right] \exp -\left[y^{2} / 2 \sigma_{y}^{2}\right]
$$

注意, 这里使用了相对论近似, 同时忽略了纵向电场的贡献。其中, $\omega$ 为辐射频 率, $\mathrm{c}$ 为光速常数, $\mathrm{q}$ 是电子电荷, $\mathrm{N}$ 是电子束团电荷数, $\mathrm{u}=(\mathrm{ux}, \mathrm{uy})$ 是观察点坐 标, $\alpha=2 \pi / \gamma \lambda, \quad b=\sqrt{\left(u_{x}-x\right)^{2}+\left(u_{y}-y\right)^{2}}, K_{1}$ 是第二类修正贝塞尔函数。为了 计算的方便, 同时也可以把微分辐射强度表示为辐射波长的函数,

$$
\frac{d I}{d \lambda}(u, \lambda)=\frac{2 \pi c}{\lambda^{2}} \frac{1}{\pi^{2}} \frac{q^{2}}{c}\left(\frac{2 \pi}{\gamma \lambda}\right)^{2} N \frac{1}{\sqrt{2 \pi \sigma_{x}^{2}}} \frac{1}{\sqrt{2 \pi \sigma_{y}^{2}}} \iint d x d y K_{1}^{2}\left(\frac{2 \pi}{\gamma \lambda} b\right) \exp -\left[x^{2} / 2 \sigma_{x}^{2}\right] \exp -\left[y^{2} / 2 \sigma_{y}^{2}\right]
$$




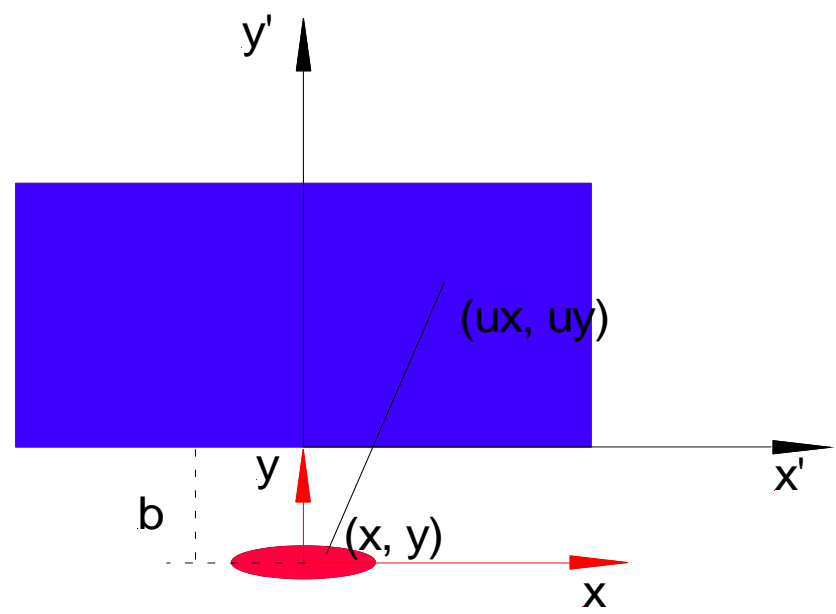

图 2.3 单边辐射示意图

假设电子束的一些基本参数如下表所示, 基于公式(2.14)可以作一些数值计算来 探讨单边衍射辐射的一些性质。

表格 2.1 衍射辐射实验中束流基本参数

\begin{tabular}{ccc}
\hline 参数 & 值 & 单位 \\
\hline 电子束能量 $\mathrm{E}$ & 4.579 & $\mathrm{GeV}$ \\
平均电流 $\mathrm{Ib}$ & 100 & $\mu \mathrm{A}$ \\
束斑水平大小 $\sigma \mathrm{x}$ & 200 & $\mathrm{um}$ \\
束斑坚直大小 $\sigma \mathrm{y}$ & 150 & $\mathrm{um}$ \\
作用参数 $\mathrm{b}$ & 1 & $\mathrm{~mm}$ \\
\hline
\end{tabular}

其中, 电子能量 $4.579 \mathrm{GeV}$ 是第二次测量所使用的能量, 平均电流 $100 \mathrm{uA}$ 接近 Hall A 的极限电流值, 该极限电流受到驱动激光的功率限制[50]。基于 OptiM[51] 对于 Hall A 束线的分析, 表中所取的电子束束斑大小是合理的。而基于坚直束 斑大小和两次测量的经验, 计算中用到的作用参数 $\mathrm{b}$ 是 $1 \mathrm{~mm}$ 。

尽管系统各元件的频率响应不尽相同, 一般认为 $400 \mathrm{~nm}-900 \mathrm{~nm}$ 对于模拟计 算来说是一个合理的辐射波长积分范围, 而且假设系统在该范围内对各波长成分 的响应无差别。在此假设下, 通过计算可以得到沿辐射屏的底边辐射的相对强度 分布曲线, 


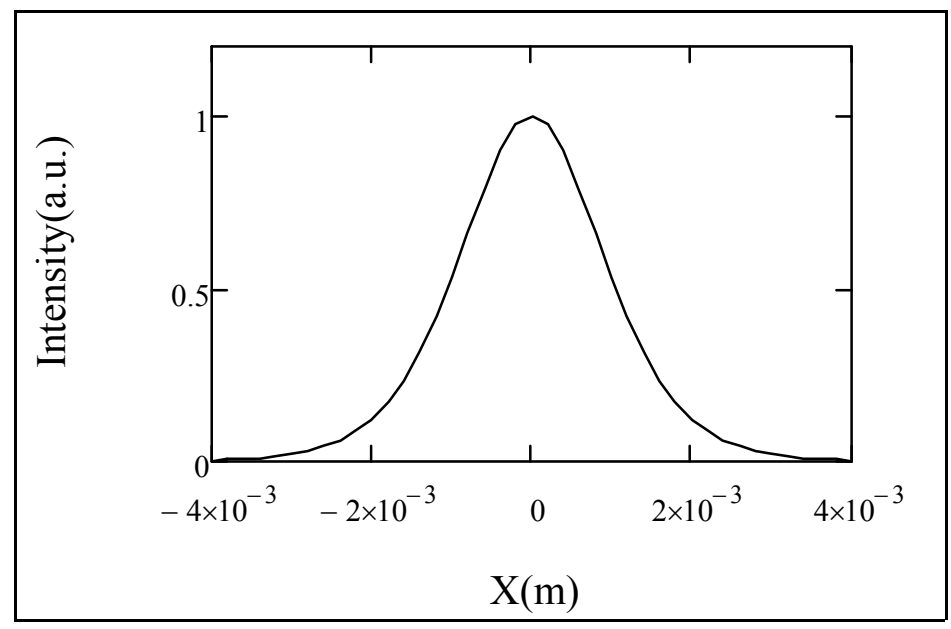

图 2.4 单边衍射辐射强度沿底边分布

可以看到, 沿底边的辐射强度近似成高斯分布。高斯分布的半高全宽由电子束分 布决定。通过计算可以证实, 沿底边辐射强度的高斯分布的半高全宽对于电子束 横向束斑大小更敏感。下面给出了横向束斑大小对于辐射分布的影响,

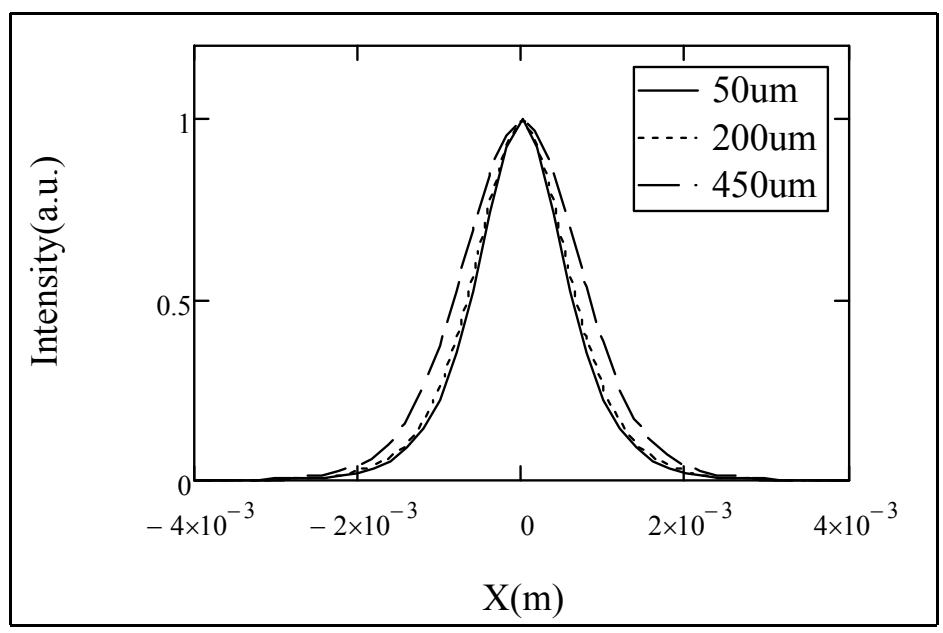

图 2.5 水平束斑对衍射辐射强度水平相对分布的影响

上面的分析为横向束斑大小的测量提供了理论的可行性, 如果能找到辐射分布和 横向束斑大小的变化关系, 自然就可以依据测量结果通过反推得到横向束斑尺 寸; 或者, 利用其他束斑大小测量手段来定标 ODR 辐射测量, 利用其非拦截性 质来做在线测量。同样，还可以通过计算得到沿屏中线坚直方向辐射强度的相对 分布, 


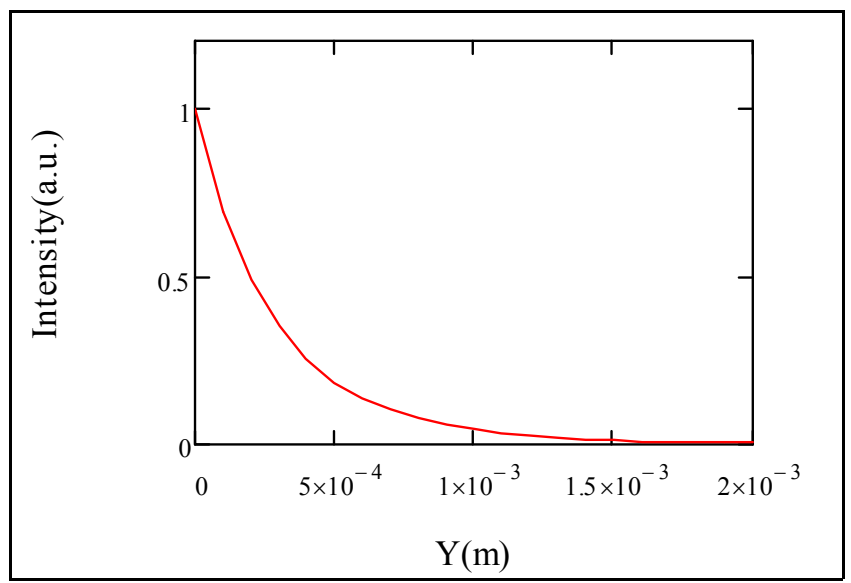

图 2.6 单边衍射辐射沿坚直方向的分布

类似地, 该指数衰减分布受束流的坚直束斑大小影响更大, 图 2.7 给出了相应 的坚直束斑大小对该分布影响的分析,

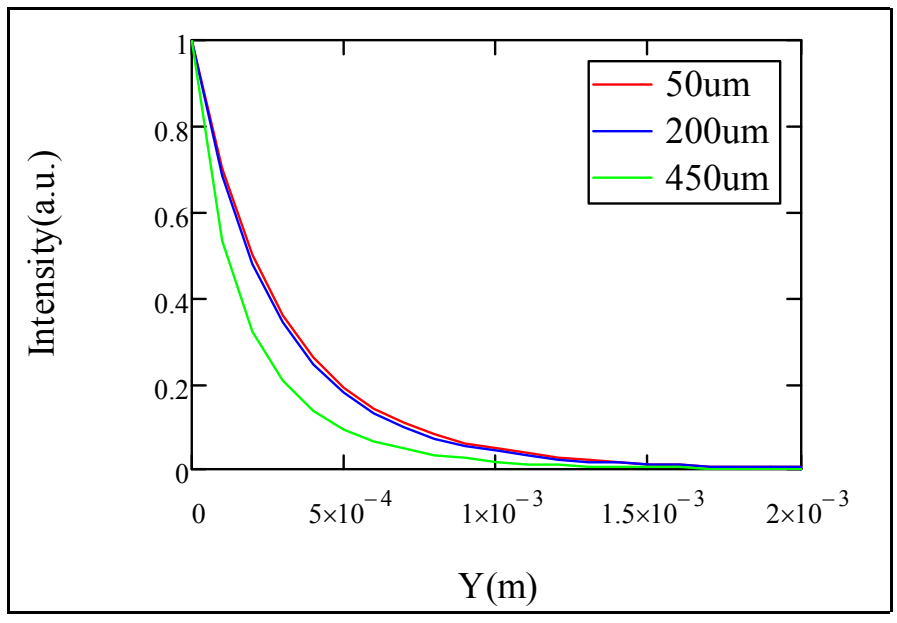

图 2.7 坚直束斑对衍射辐射强度坚直相对分布的影响

本论文中所关心的是辐射随着空间位置的分布情况，所以测量的是近场衍射 辐射。通过进一步的计算也可以得到辐射屏上辐射的二维分布,

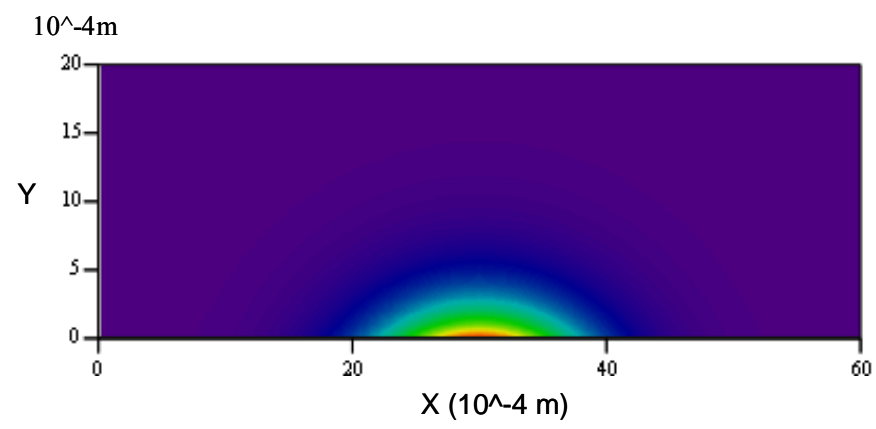

图 2.8 单边衍射辐射二维强度分布

除了辐射强度的位置分布信息，同样应该关注的还有衍射辐射的极化性质。辐射 
的极化性质来源于电子束场的极化性, 并且辐射屏对电磁场(虚拟光子)的散射不 改变其极化特性。那么在某一点水平极化辐射强度是所有电子在该处水平极化电 场作用叠加的效果[52]。基于公式(2.14), ODR 辐射水平极化分量可以表达为

$$
\begin{aligned}
\frac{d I}{d \lambda}(u, \lambda)= & \frac{2 \pi c}{\lambda^{2}} \frac{1}{\pi^{2}} \frac{q^{2}}{c}\left(\frac{2 \pi}{\gamma \lambda}\right)^{2} N \frac{1}{\sqrt{2 \pi \sigma_{x}^{2}}} \frac{1}{\sqrt{2 \pi \sigma_{y}^{2}}} \iint \frac{\left(u_{x}-x\right)^{2}}{\left(u_{x}-x\right)^{2}+\left(u_{y}-y\right)^{2}} \\
& * d x d y K_{1}^{2}\left(\frac{2 \pi}{\gamma \lambda} b\right) \exp -\left[x^{2} / 2 \sigma_{x}^{2}\right] \exp -\left[y^{2} / 2 \sigma_{y}^{2}\right]
\end{aligned}
$$

同样，ODR 辐射坚直极化分量可以表达为

$$
\begin{aligned}
\frac{d I}{d \lambda}(u, \lambda)= & \frac{2 \pi c}{\lambda^{2}} \frac{1}{\pi^{2}} \frac{q^{2}}{c}\left(\frac{2 \pi}{\gamma \lambda}\right)^{2} N \frac{1}{\sqrt{2 \pi \sigma_{x}^{2}}} \frac{1}{\sqrt{2 \pi \sigma_{y}^{2}}} \iint \frac{\left(u_{y}-y\right)^{2}}{\left(u_{x}-x\right)^{2}+\left(u_{y}-y\right)^{2}} \\
& * d x d y K_{1}^{2}\left(\frac{2 \pi}{\gamma \lambda} b\right) \exp -\left[x^{2} / 2 \sigma_{x}^{2}\right] \exp -\left[y^{2} / 2 \sigma_{y}^{2}\right]
\end{aligned}
$$

基于 表格 2.1 中的束流参数及(2.15)、(2.16)表达式, 可以计算辐射极化分量的 位置分布。下面给出沿辐射屏底边的强度分布

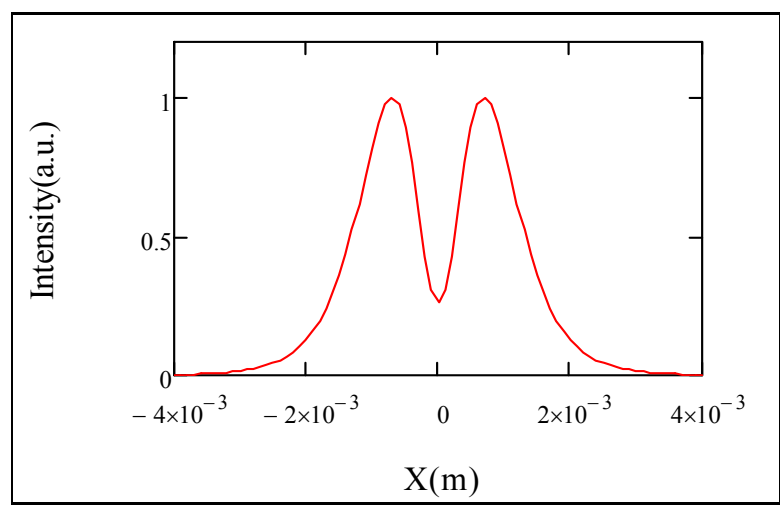

图 2.9 单边衍射辐射水平极化分量分布

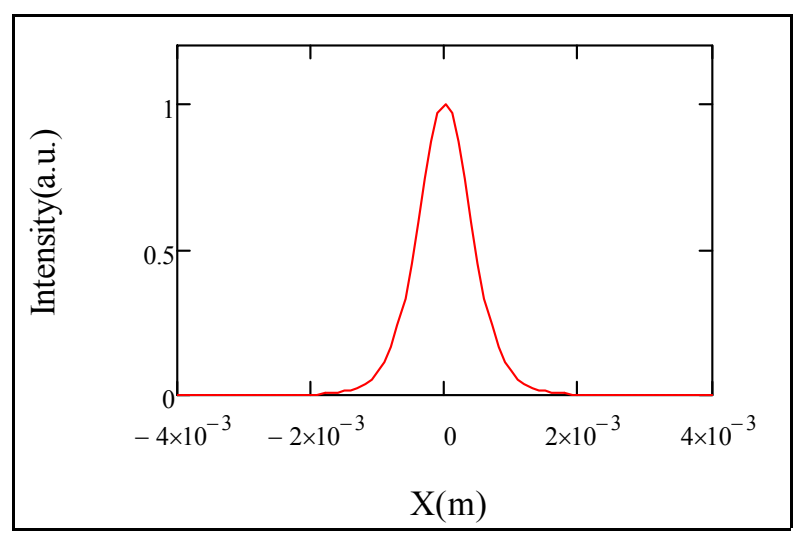

图 2.10 单边衍射辐射坚直极化分量分布 
由图可见, 水平极化分量呈对称双峰分布, 峰间距以及谷峰强度之比受束斑 两个方向大小的影响; 坚直极化分量如总强度一样呈近高斯分布, 定性的比较可 以看出, 此高斯分布具有更小的半高全宽。在后面的测量分析中会介绍, 坚直极 化分量会被利用来提高束斑测量的精度。现在分析两个极化分量如何作为束斑大 小测量的工具。首先, 对于坚直极化分量, 下图给出了其强度分布如何随着水平 束斑大小而变化

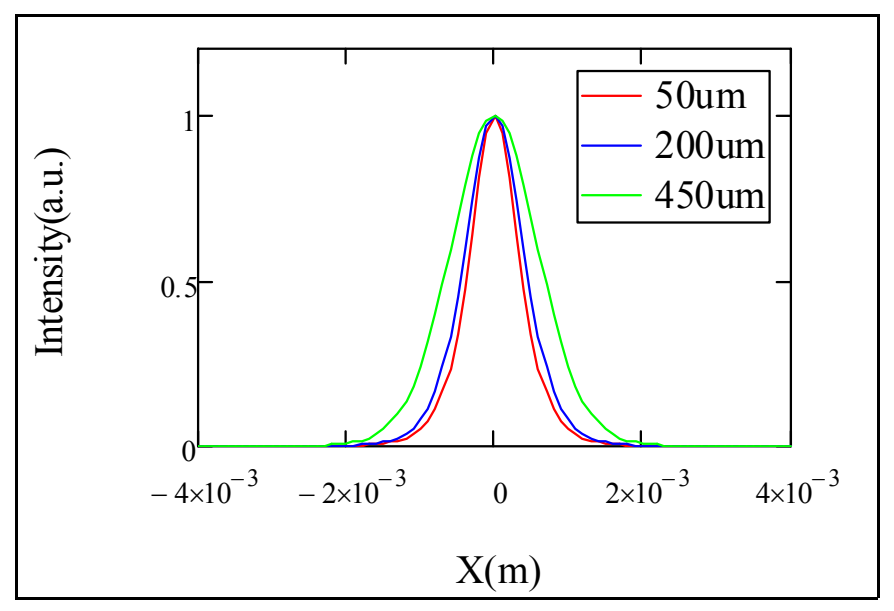

图 2.11 水平束斑对衍射辐射坚直极化分量的影响

可见, 衍射辐射坚直极化分量对于水平束斑大小有很强的依赖关系。作为定性分 析, 其依赖关系比总辐射强度分布对于水平束斑大小的依赖关系更强。这也是利 用坚直极化分量进行测量具有更高的精度的表现。同样，对于水平极化分量，下 面给出了其强度分布对于水平束斑大小的变化关系图

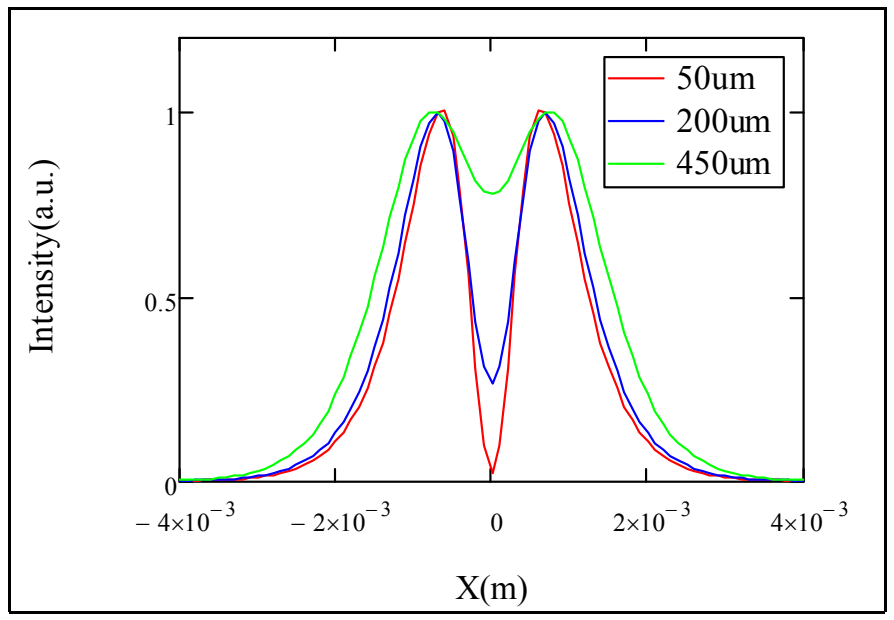

图 2.12 水平束斑对于衍射辐射水平极化分量分布的影响

可见, 水平极化分量谷峰强度比也和水平束斑大小有强烈的依赖关系。这个依赖 
关系也是一个潜在的测量束斑大小的工具。在[52]中提到, 这种明显的依赖关系 只有在水平束斑大小小于 $100 \mathrm{um}$ 的情况下出现, 这个命题只有在该篇文章中所 模拟的具体情况下成立。很明显, 在上面计算所采用的参数下, 这种关系在 50-450um 的范围内同样成立。

为了对于各个极化分量的强度分布有更直观的认识, 下面给出了二维的强度 分布图

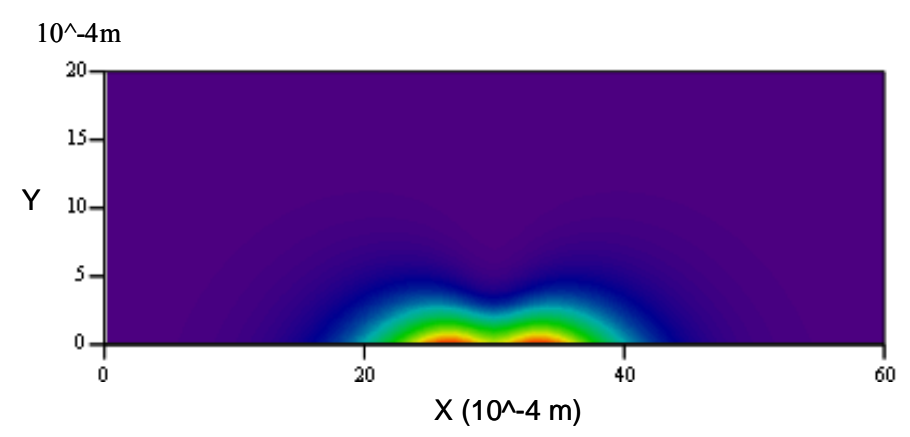

图 2.13 单边衍射辐射水平极化分量二维分布

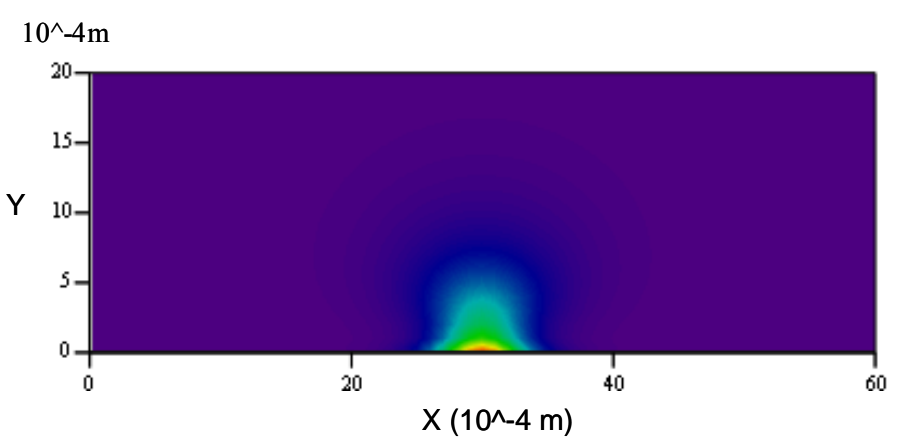

图 2.14 单边衍射辐射坚直极化分量二维分布

\section{5 辐射测量的精度}

随着 OTR、ODR 辐射在束斑大小测量方面的应用, 这类测量方式的精度成 为人们关心的问题 $[53,54]$ 。通常, 辐射测量的精度被定义为能在观察平面上被 分辨的辐射平面上最近两个电子之间的距离[55]。

由于衍射效应的普遍存在, 物平面上的一点在像平面上不会成像于一点。该 现象也会在辐射测量中出现, 但辐射还有其特殊性: 一方面, 单电子的辐射来自 于屏上一个区域而并非一个点, 这是由辐射的产生原理所决定的; 另外, 辐射光 子在沿光轴的一个很小的雉形内向外传播, 这是辐射的特性。这两方面的性质导 
致了辐射屏上一点在观察面上的像是一个 “多纳圈”。该 “多纳圈” 的形状可以 用点发散函数(Point Spread Function)来表征 [56], 实际上点发散函数就是 “多纳 圈”最大截面强度分布。考虑如下图所示的简单成像系统模型

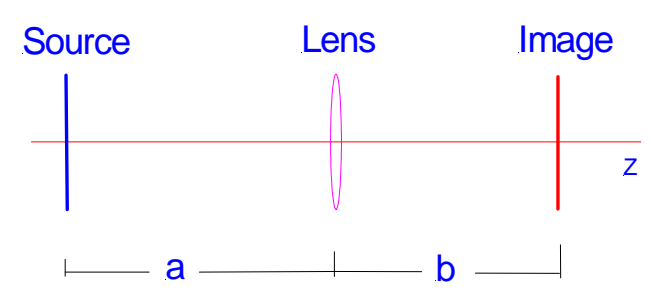

图 2.15 简化光学成像系统示意图

系统的点发散函数[57]可以表示为:

$$
f^{2}\left(\theta_{m}, \gamma, \varsigma\right)=\left[\int_{0}^{\theta_{m}} \frac{\theta^{2}}{\theta^{2}+\gamma^{-2}} J_{1}(\varsigma \theta) d \theta\right]^{2}
$$

其中, $\theta_{m}$ 是系统的最大接收角, $R_{1}$ 是表示透镜半径的变量, $\theta=R_{1} / a$, $\varsigma=k R_{i} / M, M=b / a$ 是系统的放大率。取 $M=1, \theta_{m}=0.1, \gamma=4579 / 0.511$, 并考虑中心波长 $\lambda=500 \mathrm{~nm}$ 的情况下, 像平面上 “多纳圈” 的截面分布及二维分 布为
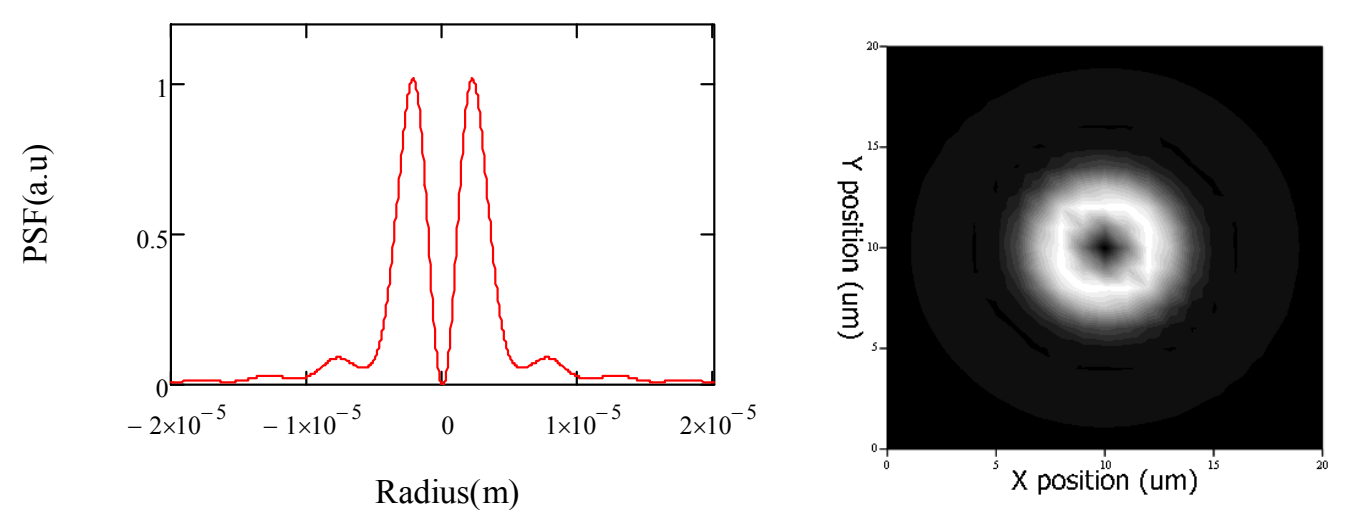

图 2.16 点发散函数一维及二维分布

如同高斯函数分布，也可以定义点发散函数的半高全宽，如果物平面上的两 点距离小于系统点发散函数的半高全宽, 那么在像平面上它们所成的像会重叠, 导致无法分辨。因此, 点发散函数的分布直接决定了系统的分辨率。如果点发散 函数具有间距大并肥胖的峰, 会直接导致不佳的系统分辨率; 反之, 系统分辨率 会得益于具有间距小并狭窄的峰的点发散函数。那么, 研究点发散函数都受那些 
参数的影响会帮助测量者做出有益于系统分辨率的选择。很明显, 点发散函数受 能量及系统接受角度的影响, 其变化规律分别如 图 2.17 及 图 2.18 所示

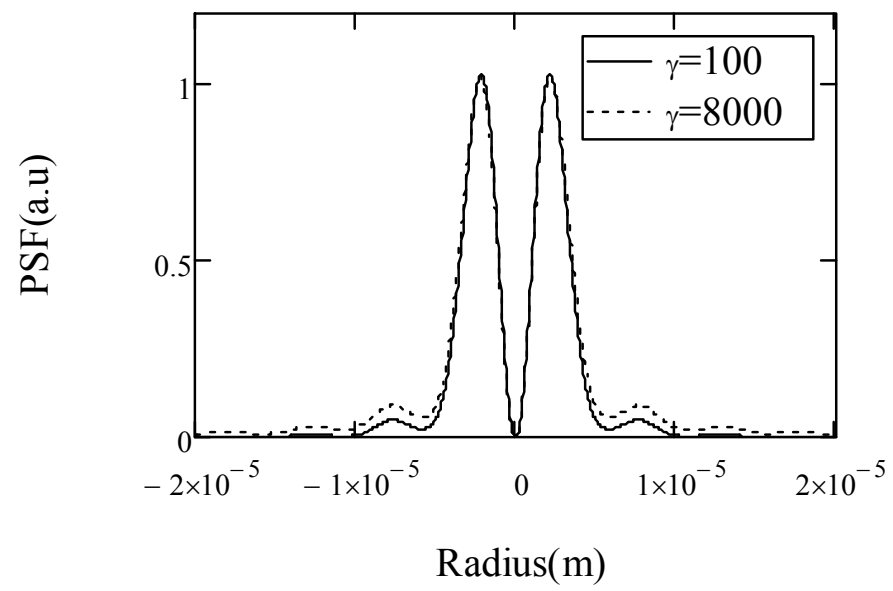

图 2.17 能量对点发散函数的影响

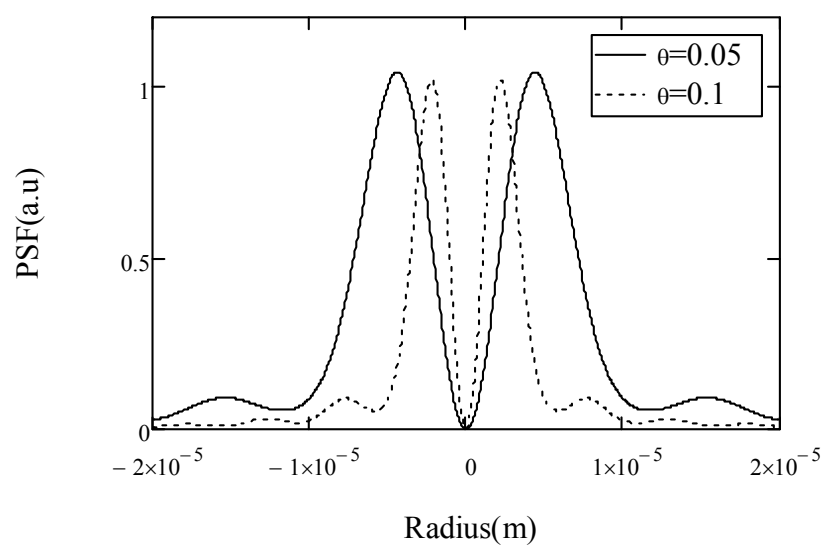

图 2.18 接收角对点发散函数的影响

因为关心的是点发散函数的相对强度分布, 所以图中所给的是归一化的强 度。很明显, 能量对于点发散函数的影响很小, 可以不予考虑。这也是 OTR 测 量能被应用于几十个 $\mathrm{KeV}$ 到很高能 $(\mathrm{GeV})$ 电子测量的原因之一 $[58,59]$ 。相反, 系统接收角度对于点发散函数的影响很大, 大的接收角下的点发散函数具有很窄 且距离很近的峰。可知, 提高系统测量精度就需要增加接收角度, 这正是在光学 系统设计中采用大口径光学原件的原因。

光路中的极化波片的作用是选择某一方向的极化电场成分, 它的存在势必也 会影响系统的点发散函数。从数学形式上来讲, 水平极化波片的作用是在电场表 达式上引入了一个 $x / \sqrt{x^{2}+y^{2}}$ 因子, 因此相当于在点发散函数里引入因子 $x^{2} /\left(x^{2}+y^{2}\right)$ 。根据该因子的变化规律(随着 $\mathrm{x}$ 的增加趋近于 1 , 随着 $\mathrm{y}$ 的增加减 
小至 0)可知, 水平极化波片有助于压缩坚直方向的点函数分布, 而对于水平方向 影响很小。因此, 在坚直束斑大小的测量中使用水平极化波片会提高分辨率。依 照相同的分析过程, 坚直极化波片有助于压缩水平点发散函数, 进而提高水平束 斑大小测量的精度。

通常, 通过增加系统接收角的方法来提高测量精度的办法是有限制的, 其限 制主要来源于现有的可用光学元件。另外一种办法也可以提高系统的精度 [57], 其基本思路是: 在系统光轴上加入一个大小合适的圆形障碍物, 其遮挡角度为 $\theta_{1}$ 的圆锥内的光线, 以此改变系统的点发散函数。在加入圆形障碍物的情况下, 系 统的点发散函数可以表示为:

$$
f^{2}\left(\theta_{m}, \gamma, \varsigma\right)=\left[\int_{\theta_{1}}^{\theta_{m}} \frac{\theta^{2}}{\theta^{2}+\gamma^{-2}} J_{1}(\varsigma \theta) d \theta\right]^{2}
$$

式中参数和一一相同。下图给出了挡光角度 $\theta_{1}$ 对于点发散函数的影响:

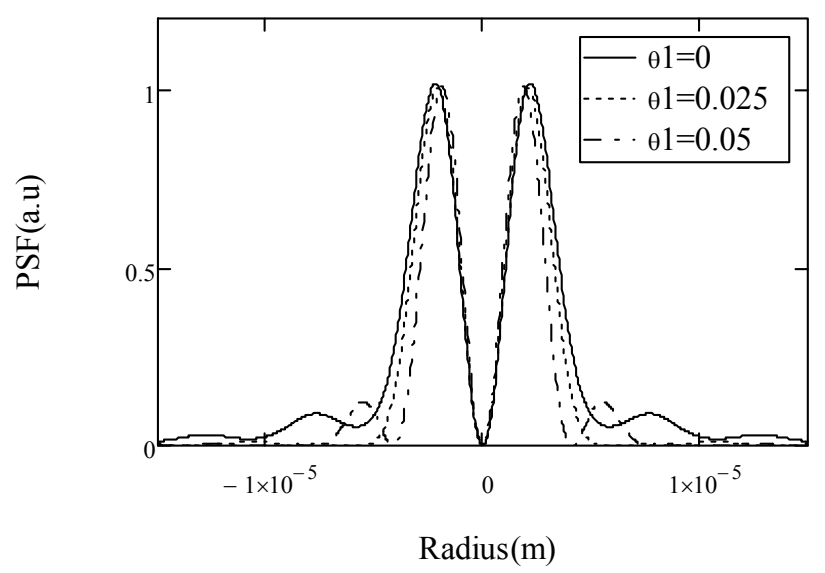

图 2.19 挡光角度对于点发散函数的影响

从图中可以看出, 在损失一部分光强的条件下, 该方法有助于压缩点发射函数进 而提高测量分辨率。

以上讨论的点发射函数是对应于单个电子的, 实际测量中, OTR、ODR 辐射截 面所成的像是对应于单电子的点发射函数和电子束电荷分布的卷积。这样也为我 们提供了将近场测量和反卷积计算相结合反推电子束截面分布的一种方法。 


\section{6 本章小结}

本章介绍了衍射辐射的理论基础--相对论电子电磁场及其与介质的相互作 用, 并将解析模型一虚拟光子模型一用于单边衍射辐射的分析, 得到了衍射辐射 分布的解析式, 通过模拟对单边衍射辐射在水平方向和坚直方向的分布有了总体 的了解，为下一步的实验奠定了坚实的理论基础; 同时, 还分析了光学系统的衍 射效应对于辐射测量精度的限制, 探讨了提高测量精度的方法, 为降低测量中的 辐射干扰提供了理论指导。 


\section{第三章 ODR实验装置}

本章主要介绍用于衍射辐射测量的装置, 以及装置中各元件基本参数及设计 考虑, 还有各元件性能对测量的影响; 同时, 也会介绍用于比较测量的坚琴系统。

图 3.1 给出了ODR试验装置的示意图。位于图中左侧的He-Ne激光、激光扩 束器、光学反射镜及可插入反射镜是该装置准直系统的重要组成部分; 位于可插 入反射镜的下游（图片右上角）的是OTR薄膜和ODR屏, ODR屏上刻有用于校 准的刻度线, 它们在步进电机的控制下可以作上下移动; 束流在OTR薄膜或ODR 屏上所产生的后向辐射会以相对于束流路径 $90^{\circ}$ 角出射至光学反射镜, 经过两 次反射后进入位于图中右下的光学成像系统; 两个消色差透镜及CV-A $50 \mathrm{CCD}$ 相 机是该辐射成像系统的主要组成部分; 在两个透镜之间, 辐射光束包络比较小处 分别放置了两个可翻转安装架 (用于安装横向偏振极化波片、纵向偏振极化波片) 和波片安装架 (用于安装各种滤波片)。下面将会介绍该装置中各组成部分的功能 及基本参数。

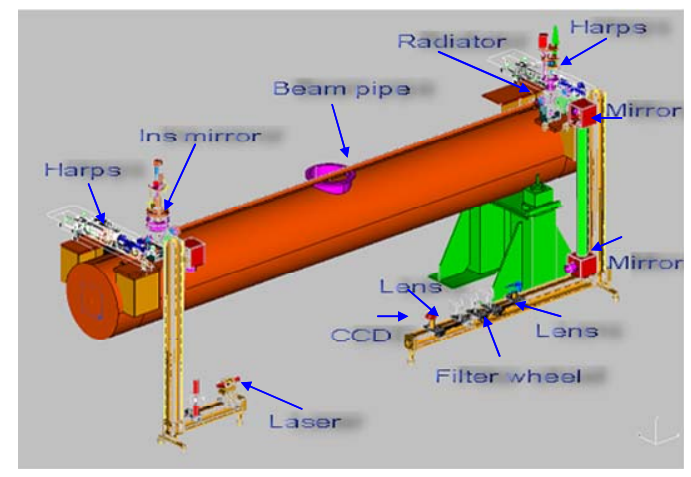

图 3. 1 ODR 实验装置总览图

\section{1 准直激光及扩束器}

准直激光及扩束器[60]被用于系统准直目的, 请结合准直过程来理解这一部 分内容。在准直的过程中, 一般要求在几十米的工作范围内束斑不会发散得很大 (几个毫米)。那么对于激光器的要求主要在于其产生的激光光学质量, 通常用激 光参数乘积(BPP)即发散角及束腰半径的乘积来表示 $[61]$ 。衍射极限的高斯光束 具有最小的参数乘积, $\lambda / \pi$, 这里 $\lambda$ 是激光波长。任意激光束参数乘积与该最小 参数乘积之比被称作为 $\mathrm{M}^{2}$ 。 
参数乘积是用于表征激光品质的固有参数, 类似与加速器中束流的发射度。 在自由空间传播时, 激光参数乘积是一个不变量。可知发射角和束腰半径成反比 的关系。在短距离内工作时一般的激光器都能很好地满足要求。为了使激光在长 工作距离的条件下始终保持小的束斑, 需要小的发散角, 那么就需要放大激光束 腰以获取小的发散角。这就是需要使用激光扩束器的原因。

准直激光及扩束器分别使用的是 Melles Griot 公司的 25 LHP 213-249 激光器 和 09 LBM 013 扩束器。该激光器产生的是波长为 $632.8 \mathrm{~nm}$ 的红光, 功率为 $0.5 \mathrm{~mW}$, 束腰半径是 $0.23 \mathrm{~mm}$, 发散角是 $1.77 \mathrm{mrad}$, 其 $\mathrm{M}^{2}$ 接近于 2 。09 LBM 013 扩束器使用的是三个非球面镜的组合设计, 它不但可以放大束腰, 而且可以调节 束腰的位置。典型的激光器如 $\mathrm{He}-\mathrm{Ne}$ 激光的束腰一般位于两个腔镜中的一个, 通过扩束器调节束腰的位置(比如位于激光器和观察点连线的中点)可以进一步 增加其可工作距离。

\section{2 可插入反射镜}

该反射镜只有在准直时由气动电机带动插入束管。由于在准直过程中该反射 镜需要上下往返多次, 对于该装置的要求就是其在来回运动中角度重复性能好, 并且适合在真空条件下工作。

\subsection{OTR薄膜及ODR屏}

为了使得二者所产生的辐射沿相同的路径进入光学成像系统, OTR 薄膜和 ODR 屏是被固定在同一个框架上的[62], 并且二者所在的平面必须很好的重合。

如图 3. 2 所示[62], 位于flag中部的即是OTR薄膜, 是在Kapton薄膜上镀铝 而成, Kapton膜厚度 $6 \mu \mathrm{m}$ 。Kapton薄膜被广泛应用于工业的各个方面, 其不仅 有很好的机械性能, 并且能抗高温耐辐射(特别是x射线)[63]。在加速器内束流冲 击的条件下, Kapton薄膜也能保持很好的形状参数。即使Kapton薄膜已有很好的 镜面特性, 但其并非很好的导体。我们知道不管是OTR辐射还是ODR辐射, 它 们都是带电粒子的场和靶上自由电子相互作用的结果。所以需要在其表面镀上一 层铝膜, 增加导电率来增进辐射。同时镀铝也进一步提升了其镜面特性, 增加后 向OTR、ODR辐射的强度。在第一次测量实验中, OTR薄膜使用的是 $8 \mu \mathrm{m}$ 厚、 
直径 0.87 英寸的铝膜, 我们将会在后面看到二者表面特性对于OTR测量的精度 的影响。

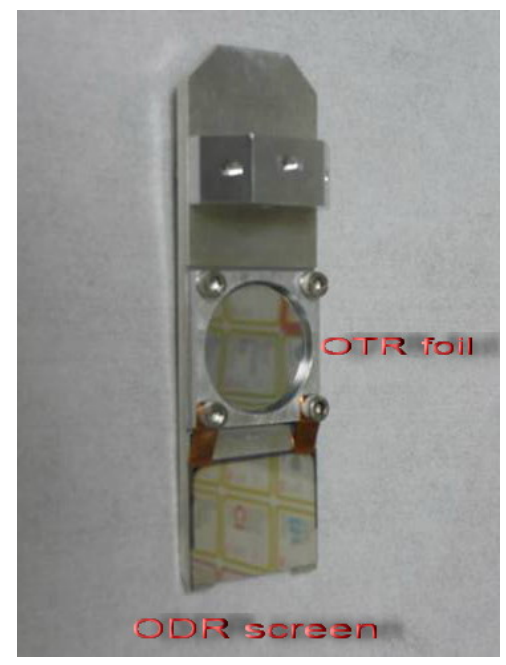

图 3. 2 OTR+ODR 辐射屏

图中 flag 下部是 ODR 屏, 是在硅片上镀铝而成, 硅片厚度 $300 \mu \mathrm{m}$ 。在硅片 上镀铝同样是两个作用: 增加导电率和提升镜面特性。ODR 辐射产生并不需要 粒子穿透辐射屏, 所以可以采用厚度不薄且价格低廉容易购买的硅片。在第一次 实验测量中, ODR 辐射屏使用的是 $1.1 \times 1.0$ 英寸、 $3 \mathrm{~mm}$ 厚手工抛光的铝片。其 对测量的影响将在后面章节展示。

\section{4 极化波片和滤波片}

使用极化波片的目的是测量辐射的不同极化分量, 而使用滤波片的目的是控 制辐射强度以及进行窄带辐射测量。

\section{4.1 极化波片}

极化波片是将非偏振或混合偏振的电磁波转换成具有单一偏振状态电磁波 的工具, 一般分为吸收性和分波性两种。根据马吕斯定律, 经过一理想的极化波 片后，非极化光束的传播效率为 $50 \%[64]$ 。在实际条件下，极化波片都有一定的 吸收损失, 导致非极化光的传输效率在 38\%左右。我们使用的是 Newport 公司的 吸收性波片 10LP-VIS-B。该波片工作在可见光波长 430-670nm 范围内, 其传输 曲线如下图所示 


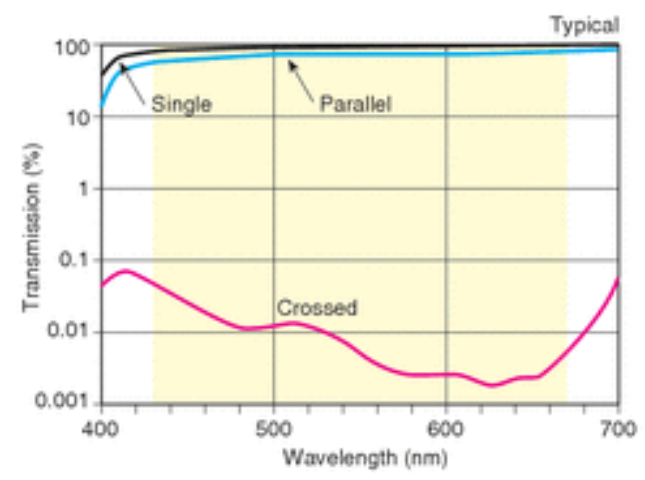

图 3.3 极化波片特性曲线

其中, 曲线 single 代表光极化方向和单个极化波片的传输线方向一致时的传 输效率; 曲线 Parallel 表示光极化方向和两个平行极化波片的传输线方向一致时 的传输效率; 曲线 Crossed 表示任意极化光通过交叉的两个极化波片时的传输效 率。

两个相同的极化波片分别被安装在可翻转架(Flip)上, 其中一个的传输线位于 水平方向, 用于吸收坚直方向偏振及传输水平方向偏振辐射; 另一个波片的传输 线位于坚直方向，用于吸收水平方向偏振及传输坚直方向偏振辐射。

\section{4 .2 滤波片}

为了防止辐射饱和以及研究辐射的频谱信息, 在光路中分别使用了中性滤波 片(Neutral Density Filter)、长通滤波片(Long-pass Filter)、短通滤波片(Short-pass Filter)及窄带滤波片(Band-pass Filter)。

理想的中性滤波片对全波段产生等量的衰减, 其衰减程度可以由几个参数来 分别表达: 光密度 $\mathrm{d}$, 经过中性滤波器后传输的相对光学功率 $\mathrm{T}=10^{-\mathrm{d}}$; 衰减数 $\mathrm{A}$, $\mathrm{A}=1 / \mathrm{T} ; \mathrm{F}$ stop, $\mathrm{F}=\log _{2}{ }^{\mathrm{A}}$ 。顾名思义, 长通、短通及窄带滤波片分别以高透射率传 输长、短及窄带的波长成分而阻止其他波长成分通过[65]。

\section{5 光学成像系统}

在光学成像系统中, 我们采用了消色差透镜[66], 原因是要尽量减小系统的 球差特别是色差来提高测量的精度。OTR 辐射和 ODR 辐射都是宽频带的辐射, 即使使用的光学元件只能传输从近红外到浅紫外的光线, 不消色差的光路都是无 
法接受的。

我们在系统中使用的是 0.5 英寸、 10 比特、单色 CV-A $50 \mathrm{CCD}$ 相机, 其 采用交织式扫描[67], 更新频率为 $50 \mathrm{~Hz}$, 信噪比 $60 \mathrm{~dB}$ 。其频率响应曲线如 下图所示:

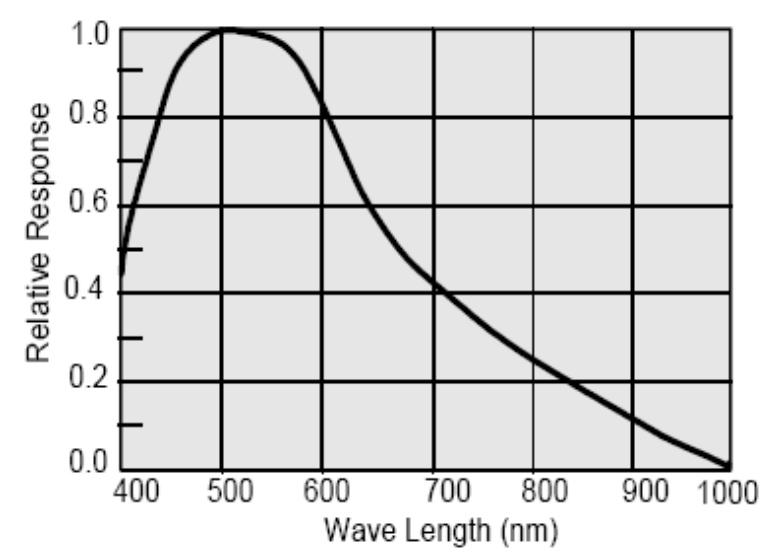

图 $3.4 \mathrm{CCD}$ 相机频率响应曲线

在该光学成像系统的设计中, 需要尽量减小衍射效应的影响。一方面, 尽量 采用大孔径的光学元件, 如 2 英寸的反射镜及消色差透镜; 另一方面, 将孔径小 的光学元件用在光学辐射包络小的地方。所以设计了双透镜成像系统, 并在空间 允许的条件小尽量将 1 英寸的偏振极化波片和滤波片放置在两个透镜之间。

光学成像系统的设计计算并不难, 主要是基于透镜成像公式的一些基本计 算, 但是对于辐射成像系统的设计也有其特殊性。设计中最重要的一个参量是系 统的视场(Field of View)大小, 即多大的视野范围会被成像于观测器 (CCD 相机) 上。如在第一次实验测量中采用的视场大小是 $\sim \times 3$ 毫米, 而 $\mathrm{CCD}$ 相机是 640 $\times 480$ 像素、10 微米/像素, 所以总的成像系统的放大率是 1.6。使用的设计如下 图, L1 $=1.03 \mathrm{~m}, \mathrm{fl}=0.25 \mathrm{~m}, \mathrm{~L} 2=0.33 \mathrm{~m}, \mathrm{~L} 3=0.09 \mathrm{~m}, \mathrm{f} 2=0.075 \mathrm{~m}, \mathrm{~L} 4=0.45 \mathrm{~m}$ 。系 统的放大率 $\mathrm{M}=(\mathrm{L} 4 / \mathrm{L} 3)^{*}(\mathrm{~L} 2 / \mathrm{L} 1) \sim 1.6$ 。如果需要改变系统的视场大小, 移动任何 一个透镜并适当调整 CCD 相机的位置可以达到目的, 很明显改变第二个透镜的 位置能更有效调整系统的放大率。

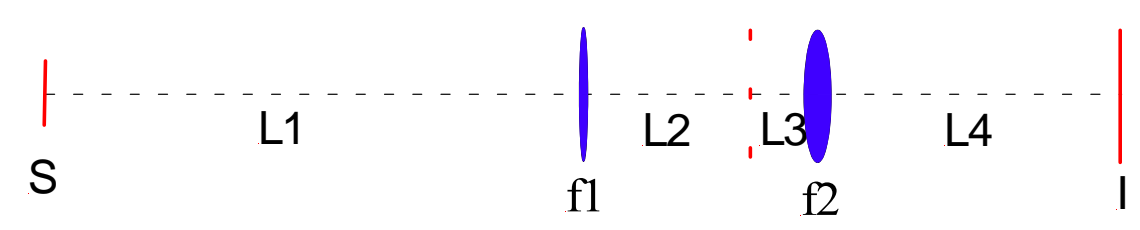

图 3.5 光学成像系统示意图 


\section{6 系统准直}

做系统准直的目的是：将激光路径准直到和电子束路径重合，然后用激光束 来作光学系统的准直, 使得激光在辐射靶上的斑点在 CCD 相机上成清晰的像, 实验测量时辐射电磁波束将会沿相同的路径成像于 CCD 相机。准直的示意图如 下，为了方便该图和实物不成比例，下面是准直的过程介绍：

1. 固定激光器, 置入可插入反射镜 M1 及 ODR 辐射屏, 调节位于激光器和可插 入反射镜 M1 之间的镜子，同时调节辐射靶之后的光学元件，使得激光在 ODR 屏上的斑点在 CCD 相机上成清晰的像; 进一步调节位于激光器和可插入反射镜 之间的镜子, 使得靶上的激光斑点和电子在靶上应该在的位置(由机器准直人员 提供)重合，再次调节光学系统，使该点的像清晰。

2. 撤回 ODR 屏, 置入下游 $30 \mathrm{~m}$ 处的反射镜 $\mathrm{M} 2$, 这是在 M2 上并不一定能看到 激光束斑, 这样的话就需要调节 M1 之前的反射镜。

3. 如果 M2 上的激光斑点与电子束在该处的位置不重合, 表明激光在 ODR 靶处 虽然位置正确但角度偏离电子路径, 进一步调节 M1 之前的镜子, 同时调节激光 扩束器, 扩束并将束腰外推至十多米处, 移动光斑到电子在 M2 上的位置。

4. 插入 ODR 屏, 回到步骤 1, 重复 1-3 步几次, 直到激光在 ODR 屏上的位置和 角度都正确无误。

5. 再次准直光学成像系统。

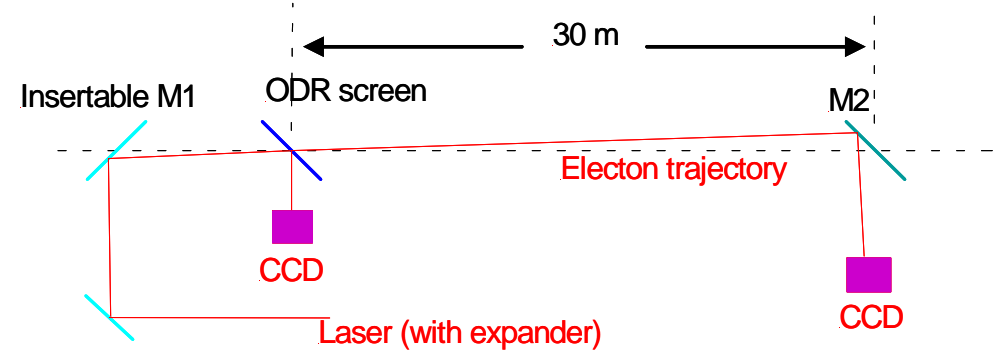

图 3.6 系统准直示意图

\section{7 坚琴测量}

从 ODR 实验装置图中可以看到, 在可插入反射镜和 ODR 辐射靶附近分别有 一个坚琴测量装置。第二个坚琴上的测量结果将会和 OTR、ODR 的测量结果进 
行比较。在 Jlab CEBAF 上使用了多种结构的坚琴测量装置 $[68,69]$, 下面来看看 该坚琴装置的结构和工作方式。

如下图所示, 该坚琴主要由 3 条 $25 \mu \mathrm{m}$ 的铇丝所组成。铇丝 $\mathrm{x}$ 在坚直方向, $\mathrm{u} 、 \mathrm{v}$ 均与 $\mathrm{x}$ 成 45 度角。在气动电机的驱动下, 该坚琴沿水平方向运动并扫描电 子束横截面。各铇丝上电荷随时间的变化曲线、即电子束某一方向的电荷分布将 会被记录并分析。如铜丝 $\mathrm{x}$ 给出电子束 $\mathrm{x}$ 方向的电荷分布参数 $\sigma_{\mathrm{x}}$ 。电子束在 $\mathrm{y}$ 方向的分布参数由下面关系式给出[70],

$$
\sigma_{y}^{2}+\sigma_{x}^{2}=\sigma_{u}^{2}+\sigma_{v}^{2}
$$

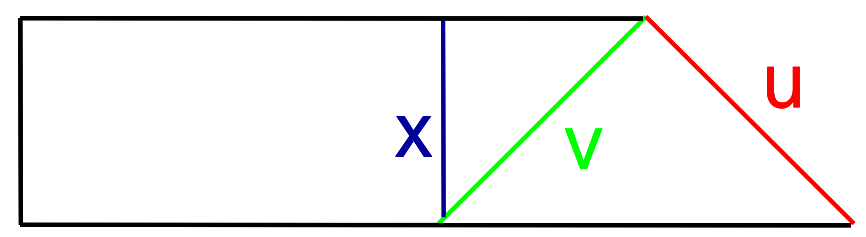

图 3.7 坚琴结构示意图

\section{8 本章小结}

本章介绍了衍射辐射测量装置中用于准直的激光及扩束器、OTR 及 ODR 辐 射屏、辐射光学成像系统和坚琴测量系统。一方面给出了装置的总体设计, 使得 辐射测量的思路更具体, 另外也给出了具体元件的选择标准。 


\section{第四章 实验测量}

CEBAF 可以工作在两种束流模式下: 一是调试机器时的束流工作模式, 称 之为调机模式(Tune Beam), 该模式下束流有宏脉冲结构, 占空比因子(Duty Fact) 为 $1.5 \%$ 。另外就是核物理和高能物理实验所要求的连续波模式, 主机器的脉冲 频率是 $1497 \mathrm{MHz}$, 各个物理实验室则是 499MHz[39]。两种工作模式下微脉冲结 构没有变化, 都是由驱动激光的结构所决定的, 驱动激光的功率则决定了单脉冲 电荷量。

测量过程涉及到这两种束流模式下的坚琴测量、OTR 测量以及 ODR 测量。 依照测量进行的顺序，下面分别介绍实验测量中的每一部分。

\section{1 空间定标}

根据光学系统的设置及 CCD 相机的参数, 可以计算系统的放大率和视场大 小, 所以也就能知道究竟 $\mathrm{CCD}$ 相机上的像所对应物的实际大小。但是, 这种办 法的精确度受到限制，所以一般不采用该种办法。限制主要来自有三个方面：一 是搭建光学系统时往往和设计计算的值有偏差; 另外光路中使用的并非理想光学 元件; 最后, 即是我们对光学系统进行尺寸测量, 进而在测量数据的基础上计算, 也会带入测量误差。

因此, 我们考虑在 OTR + ODR 屏上设置一个定标尺。很明显, 小的定标尺会 引入更大的系统误差。然而, 基于初步计算, 系统的视场大小在 4 7mm 范围内, 所以该定标尺又不能大于视场大小。另外, 步进电机的上下活动范围有限, 为了 进行 OTR 测量, 必需能够将 OTR 薄膜中心移到束流中心, 而为了进行 ODR 测 量, 必需能够将 ODR 屏底边移到束流中心上一定距离处, 加上定标尺的尺寸, 总的纵向尺寸必需小于步进电机的移动范围。综合以上原因，我们在 OTR 薄膜 和 ODR 屏之间刻写了方格状的定标尺。

图 4. 1 中定标尺上的小方格的大小是 $2.83 \times 2 \mathrm{~mm}$ 。因为屏和束流径迹成 45

。角, 从束流的方向看, 小方格呈 $2 \times 2 \mathrm{~mm}$ 的正方形; 同样, 沿后向辐射产生的 方向看也应该是一个 $2 \mathrm{~mm}$ 的正方形方格。定标的过程是：利用准直激光照射该 定标尺, 并将 $\mathrm{CCD}$ 相机聚焦到辐射屏, 记录下辐射屏清晰的像, 然后进行分析计 
算。

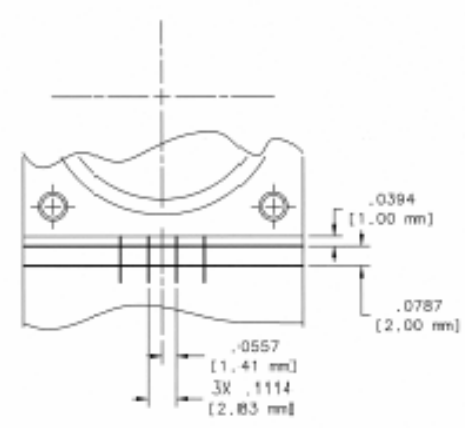

图 4.1 定标尺 CAD 示意图

图 4.2 是第一次测量中所做的空间定标

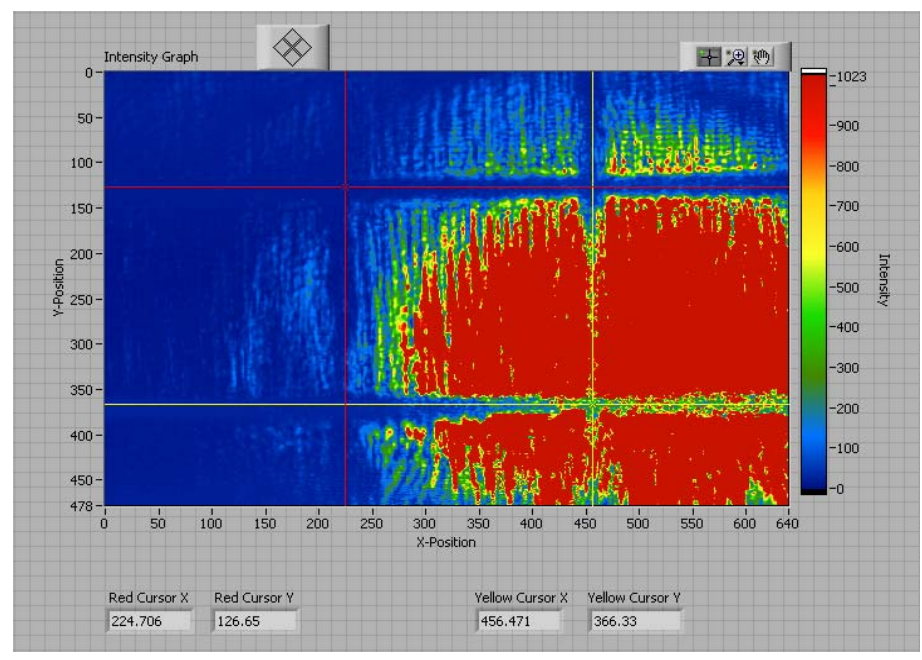

图 4.2 定标测量图 1

在测量过程中使用了 LabView 来记录并分析结果。图中光标给出了小方格在 $\mathrm{CCD}$ 相机上成像所在的范围, $\mathrm{x}$ 方向从 224.7pixel 到 456.5pixel, y 方向从 126.7pixel 到 366.0pixel。所以, 定标因子（即 CCD 相机上每个像素代表的实际 尺寸）分别是在 $\mathrm{x}$ 方向 $8.65 \mathrm{um} / \mathrm{pixel}$ 、在 $\mathrm{y}$ 方向 $8.36 \mathrm{um} / \mathrm{pixel}$ 。可以知道视场大 小实际上等于 $\mathrm{CCD}$ 大小乘以定标因子, 在此定标的基础上, 计算得到的视场大 小为 $5.54 \times 3.99 \mathrm{~mm}^{2}$ 。

此次定标的结果告诉我们, $\mathrm{x} 、 \mathrm{y}$ 方向上的定标因子差别并不大, 并且, 我们 主要关心的是水平方向上的测量, 因此并不需要精确的坚直方向的定标。所以, 在第二次实验测量中，使用了一种更简便的定标方法。具体过程如下

1. 调节激光扩束器, 使得其在 ODR 屏处的束斑约几个毫米

2. 向下移动 $\mathrm{ODR}$ 屏, 直到其底边能在 $\mathrm{CCD}$ 相机上成清晰的像, 保存 $\mathrm{CCD}$ 上的 


\section{图像}

3. 继续向下移动 ODR 屏 3mm, 确保此时其底边还能在 CCD 相机上成清晰的像, 保存 CCD 上的图像

下面是该过程中所记录的 CCD 图片，根据它们可以计算其定标因子。
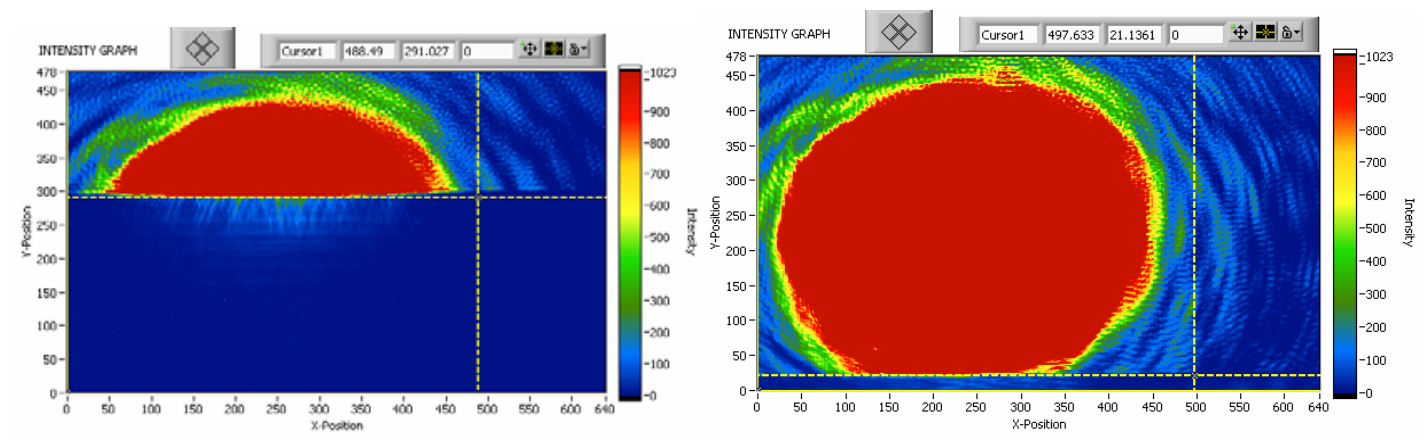

图 4.3 定标测量图 2

两图上光标位置显示 ODR 屏的底边是从 291.0pixel 移动到了 21.1pixel。因 此, 经过调整后的光学系统的定标因子是 $11.12 \mathrm{um} / \mathrm{pixel}$, 视场大小是 $7.1 \times$ $5.3 \mathrm{~mm}^{2}$ 。系统的放大率从最初的 1.16 降到了约 0.9 。

从左图可以看到, 本来笔直的屏底边在 $\mathrm{CCD}$ 相机上并不是一条直线。我们 知道这样的现象来自于成像系统的畸变 [71], 并根据畸变的形状以及发生在 CCD 相机的上部可以判断其为正的径向畸变— “针垫” 畸变[72]。纠正该种畸变的 办法就是采用远心光学成像系统[73]。

这里所说的畸变的定义是, 实际光学像点到中心的距离与理想系统所成像中 点到中心的距离的相对差别。下图给出了分别具有正负径向畸变的光学系统所成 的像。

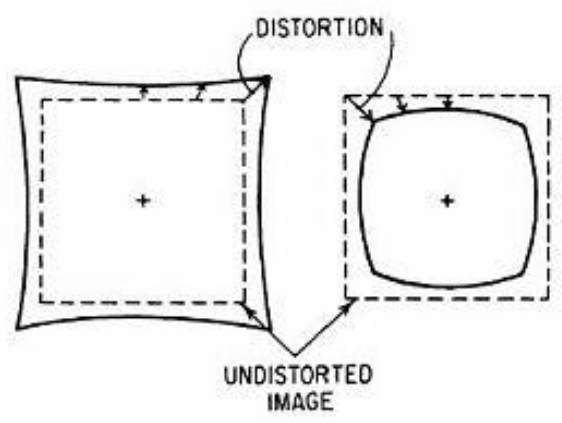

图 4.4 正负径向畸变示意图 
远心的意义是指透镜聚焦在无限远处。因此远心光学成像系统被分成三类: 物镜远心系统、目镜远心系统和双远心系统。但实际使用的远心系统的物镜和目 镜都是聚焦在有限距离处, 但物距和相距可以在很大范围内变动而对成像质量没 有大的影响。

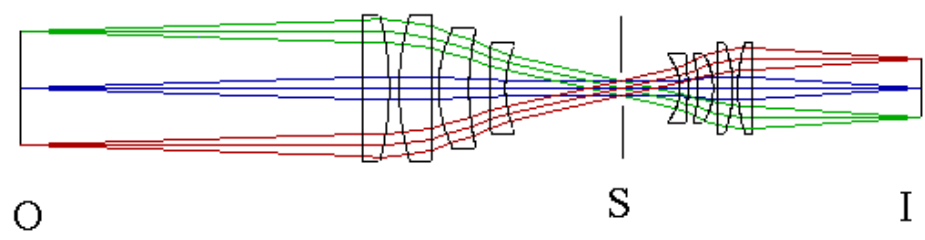

图 4.5 远心成像系统光线图

这是一个常见的商业用远心系统的光线图[74]。物位于 $\mathrm{O}$ 平面, 像位于 $\mathrm{I}$ 平 面。使用该系统可以用来观测底面和第一块透镜等大的圆柱范围内的物体。因此, 远心系统对于物体的大小有限制。在允许范围内的物体, 会被该系统各向等量缩 放成像在 I 平面。可以理解, 如果使用这样的远心成像系统, 则不会出现直线不 直的畸变现象, 从而提高测量的精确度。

\section{2 坚琴测量}

在 Jlab 加速器上使用到的坚琴装置按材料分类有两种: 碳纤维坚琴和铇丝坚 琴。按信号采集方式则可以分为前级放大坚琴和光电倍增管坚琴 [68]。所有这些 坚琴都具有相同的功能, 就是提供束流的电流强度截面分布, 都是通过细丝扫描 束流截面时记录细丝上的电荷及细丝位置来测量的。如果作细丝位置与细丝上电 荷的关系图, 就可以得到如下的示意图。图中三个峰分别对应于三条细丝, 并可 以通过高斯拟合得到标准偏差。

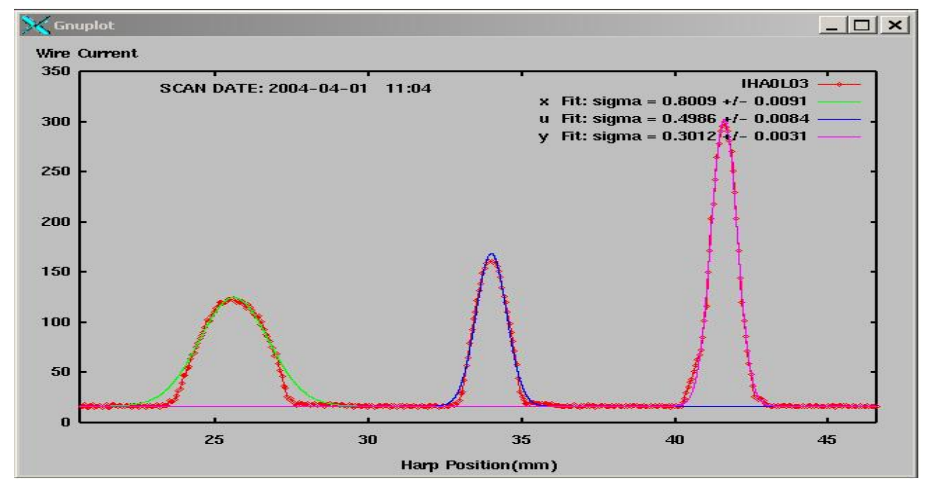

图 4.6 坚琴测量及拟合结果图 
前级放大坚琴有三条牢固安装的铇丝, 通过不锈钢杆安装在步进电机上。在 步进电机的带动下匀速沿着一个方向扫描电子束的截面。当坚琴扫过电子束截面 时, 细丝会收集少量的电荷, 该电荷通过真空 $\mathrm{BNC}$ 接头以及双绞线传输到前级 放大。前级放大的信号最后会被传输到模数转换模块。

在光电倍增管坚琴中, 信号的传输不是直接的。在这种坚琴装置中, 光电倍 增管代替了前级放大被安装在细丝的下游。当细丝扫描束流时, 束流和细丝的作 用产生二次电子, 这些二次电子被下游的光电倍增管收集。光电倍增管所收集的 二次电子正比于细丝所拦截的束流电荷。

坚琴测量装置在 CEBAF 机器上一共有 31 套, 它是一个常规的束流截面电荷 分布的工具。因为编号为 IHA1C18B 的坚琴紧靠 ODR 辐射屏的位置, 所以它是 一个可选择的校准测量方法。另外一个可选择的校准工具是 OTR 测量, 最终的 选择会基于对二者准确度的比较。下面是坚琴测量的示意图

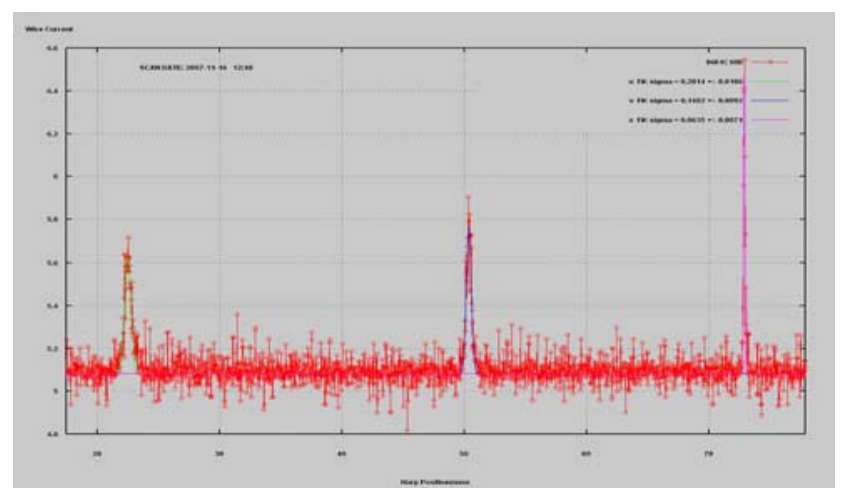

图 4.7 坚琴测量结果

通过对 $\mathrm{Q}$ 铁的强度进行调节, 束流可以运行在 10 个不同束斑大小的情况下。 每一种情况下, 我们都利用多种束斑测量手段进行了测量比较。下面是坚琴测量 的结果

表格 4.1 四极铁扫描下坚琴测量结果

\begin{tabular}{ccc}
\hline Q scan/Harps & $\sigma_{\mathrm{x}}$ & $\sigma_{\mathrm{y}}$ \\
\hline Q1 & 329 & 154 \\
Q2 & 350 & 115 \\
Q3 & 303 & 129 \\
Q4 & 266 & 146 \\
Q5 & 258 & 131 \\
\hline
\end{tabular}




\begin{tabular}{ccc}
\hline Q6 & 209 & 121 \\
Q7 & 204 & 104 \\
Q8 & 182 & 141 \\
Q9 & 148 & 135 \\
Q10 & 126 & 154 \\
\hline
\end{tabular}

表格 4. 1 是在 10 个不同的 $\mathrm{Q}$ 铁参数下使用坚琴测量得到的水平和坚直方向 的束斑大小。对应于坚琴测量，我们在相同的条件下也进行了 OTR测量和ODR 测量, 结果在后面给出。

\section{3 OTR测量}

相对于坚琴测量, OTR 测量更大程度上可以被我们控制，因此也有更大的空 间来改善测量精度。比如, 我们用镀铝的 Kapton 薄膜代替了铝膜, 更好的镜面 特性为我们带来了更高的精度，从下面记录的图片就可以看出。第一个图测量时

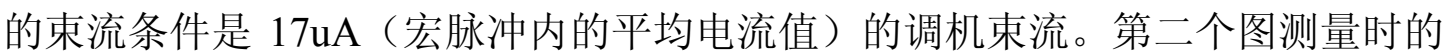
束流条件是 $10 \mathrm{uA}$ 的调机束流。

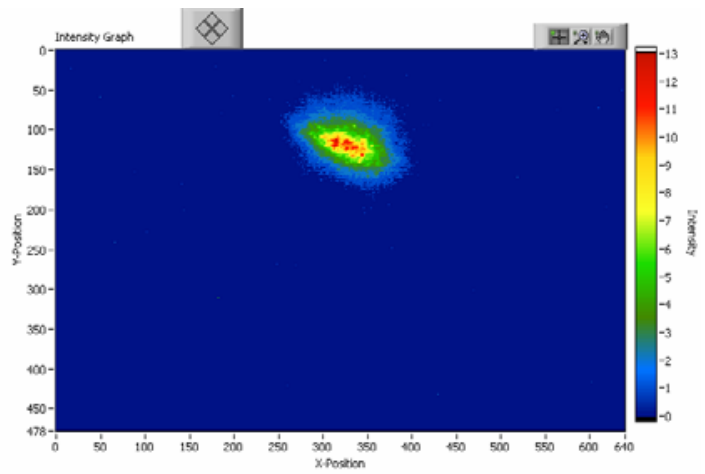

图 4.8 铝膜 OTR 测量图

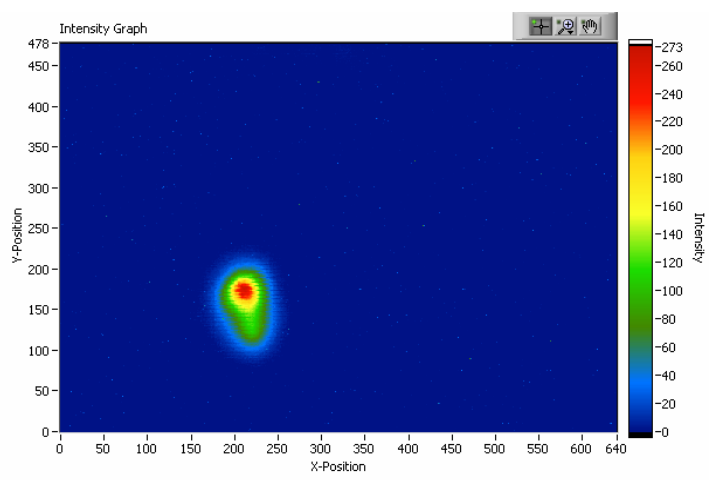

图 4.9 镀铝 Kapton OTR 测量图 
如前所述, 为了防止发生 OTR 薄膜被束流损坏的情况, ODR 屏（镀铝硅片） 也被保留了用作 OTR 辐射屏的可能性。实验过程中的谨慎操作保证了我们并没 有出现 OTR 薄膜被损的情况，另外这也得益于 Kapton 薄膜非常好的机械性能。 但是, 在安装过程中 OTR 薄膜和 ODR 屏并不是处于理想的同一平面, 这样导 致成像光路并不能很好地同时搭配 OTR 薄膜和 ODR 辐射屏进行工作。这样, 我们准直过的光路只能很好地为 ODR 屏成像。我们同时给出了 OTR 薄膜和 ODR 屏上产生的 OTR 辐射近场像。 1 和 2 分别是在电流强度为 $1 \mathrm{uA}$ 和 $10 \mathrm{uA}$ 的调机 束流时的测量, 所得到的束斑大小分别是 $\sigma_{\mathrm{x}}=97 \mathrm{um}, \sigma_{\mathrm{y}}=242 \mathrm{um} ; \sigma_{\mathrm{x}}=184 \mathrm{um}$, $\sigma_{\mathrm{y}}=298 \mathrm{um} 。 3$ 和 4 分别是在电流强度为 $1 \mathrm{uA}$ 和 $5 \mathrm{uA}$ 的调机束流时的测量, 所得 到的束斑大小分别是 $\sigma_{\mathrm{x}}=145 \mathrm{um}, \sigma_{\mathrm{y}}=161 \mathrm{um} ; \sigma_{\mathrm{x}}=158 \mathrm{um}, \sigma_{\mathrm{y}}=163 \mathrm{um}$ 。因为相同的原 因, 不同的束流电流强度情况下, OTR 薄膜产生的像的大小发生了变化。作为 ODR 测量的校准工具, 必备的属性之一就是不同电流强度条件下给出相同的束 斑大小。因此, ODR 屏上的 OTR 辐射被保留为除了坚琴测量外的可选择的校准 工具。

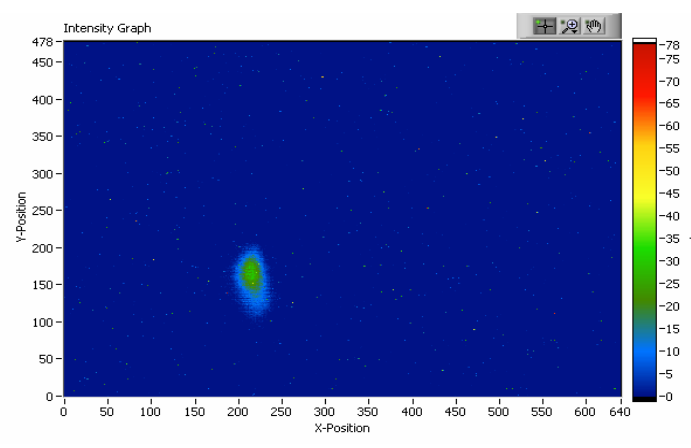

(a)

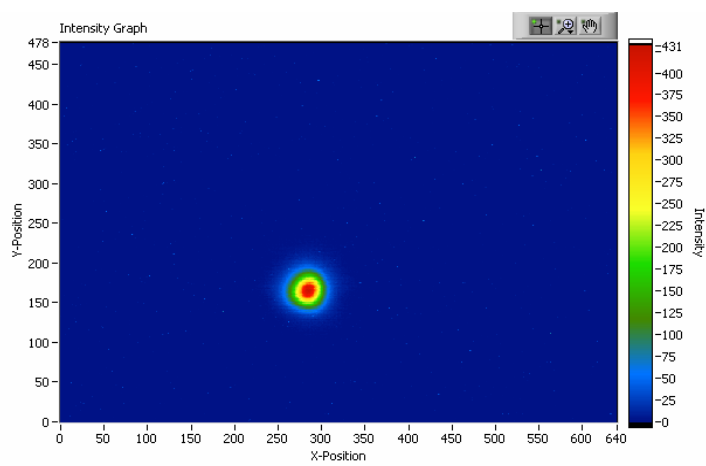

(c)

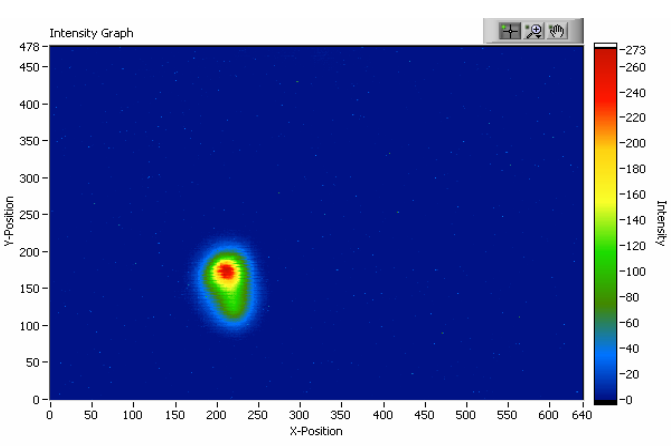

(b)

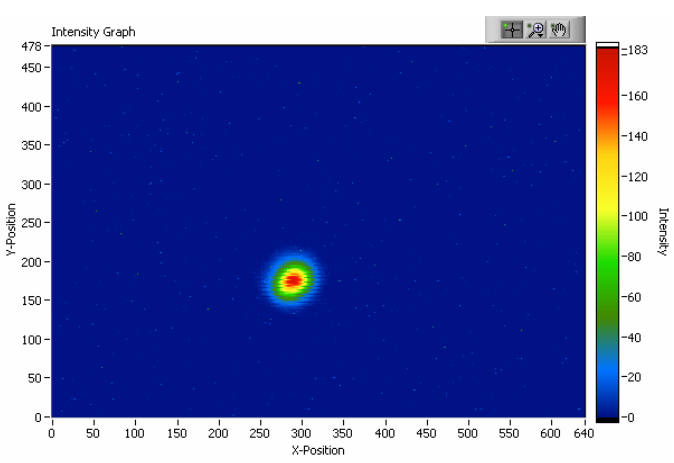

(d)

图 4.10 OTR 测量对比: 镀铝 Kapton 靶 OTR 测量 a. $1 \mathrm{uA}$ 电流 b. 10uA 电流;

镀铝硅片靶 OTR 测量 c. $1 \mathrm{uA}$ 电流 d. $5 \mathrm{uA}$ 电流 
除了靶材料的变化外，我们也在光路中使用了两个正交线性极化波片，通过 其对点发散函数的改变来提高测量的精度。下面的测量是在调机束流电流强度是 $5 \mathrm{uA}$ 的同一条件下进行的, 都使用了光学密度为 0.1 的中性滤波片, 辐射产生于 ODR 屏。1 中没有使用极化波片, 2 中使用了水平极化波片, 3 中则使用了坚直 极化波片。作为定性的分析，我们看到 2 中水平极化波片的作用是 “压缩” 束斑 坚直分布, 使得坚直束斑更接近真实的束斑大小; 3 中坚直极化波片的作用则是 “压缩”束斑水平分布，使得水平束斑更接近真实的束斑大小。

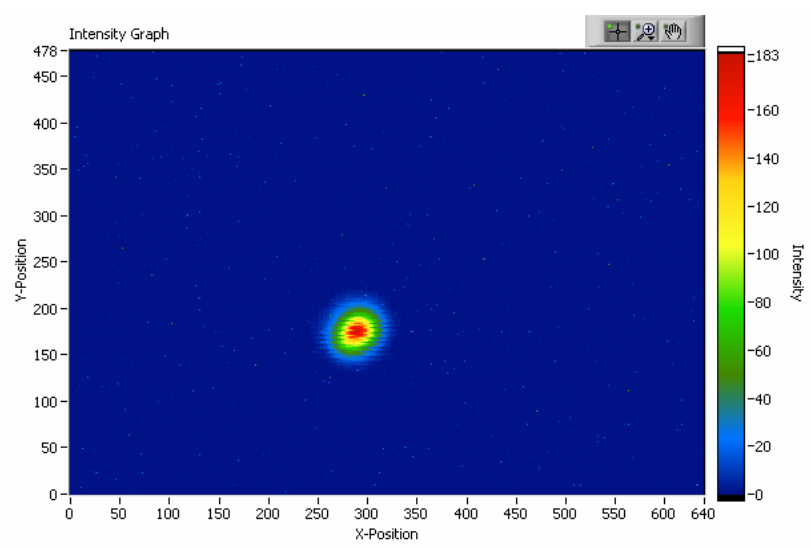

图 4. 11 镀铝硅片 OTR 总辐射强度

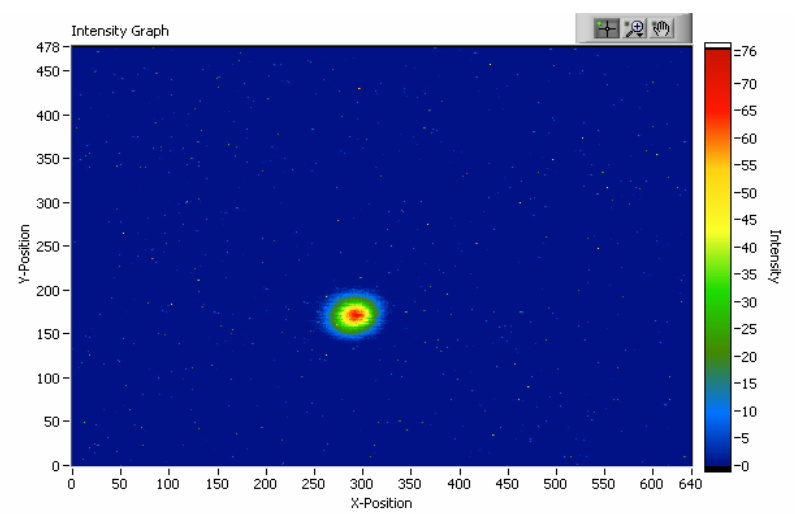

图 4.12 镀铝硅片 OTR 辐射水平极化分量

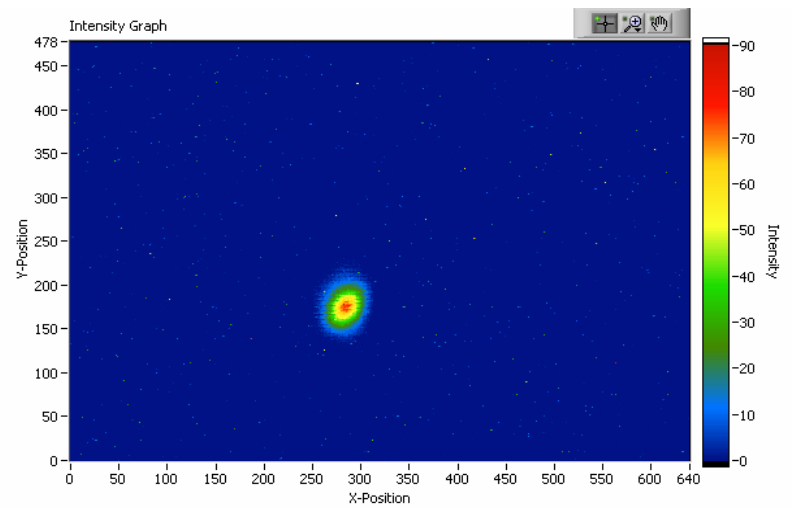

图 4. 13 镀铝硅片 OTR 辐射坚直极化分量 
图中所得到的束流尺寸分别是: $\sigma_{\mathrm{x}}=158 \mathrm{um}, \sigma_{\mathrm{y}}=163 \mathrm{um} ; \sigma_{\mathrm{x}}=153 \mathrm{um}, \sigma_{\mathrm{y}}=133 \mathrm{um}$; $\sigma_{\mathrm{x}}=127 \mathrm{um}, \sigma_{\mathrm{y}}=164 \mathrm{um}$ 。对应于 $\mathrm{Q}$ 铁扫描下做的坚琴测量, 我们也进行了 OTR 测 量。下表能够更好的反映极化波片在束斑测量中的提高测量精度的作用。

表格 4.2 四极铁扫描下 OTR 测量结果

\begin{tabular}{ccccccc}
\hline Q scan OTR & \multicolumn{2}{c}{ Total } & \multicolumn{2}{c}{ Hor } & \multicolumn{2}{c}{ Ver } \\
\hline 5uA Q1 & 390 & 142 & 383 & 120 & 342 & 154 \\
5uA Q2 & 362 & 144 & 365 & 125 & 333 & 166 \\
5uA Q3 & 342 & 150 & 353 & 121 & 312 & 166 \\
5uA Q4 & 323 & 150 & 320 & 134 & 276 & 165 \\
5uA Q5 & 292 & 152 & 291 & 127 & 265 & 171 \\
5uA Q6 & 269 & 160 & 283 & 137 & 245 & 174 \\
5uA Q7 & 257 & 161 & 262 & 139 & 223 & 178 \\
5uA Q8 & 243 & 168 & 246 & 150 & 209 & 182 \\
5uA Q9 & 223 & 182 & 220 & 145 & 189 & 186 \\
5uA Q10 & 188 & 182 & 200 & 151 & 164 & 188 \\
\hline
\end{tabular}

其中, Total 表示没有极化波片的测量, Hor 表示有水平极化波片的测量, Ver 表 示有坚直极化波片的测量。

\section{4 ODR辐射测量}

OTR 测量有着多种作用, 这里要说的是其中很重要的一项一一作为束流位置 的测量。基于实验测量初期进行的 OTR 测量, 我们可以判读束流是否在束管中 心附近, 是否必要调整束流径迹来方便 ODR 测量。然后下一步需要选择的参数 则是 ODR 辐射的作用因子 (即束流中心离 ODR 屏底边的距离)。基于带电粒子 束电场沿径向的分布, 我们知道辐射靶离电子束中心越近产生的辐射越强 (为了 产生可观的波长为 $\lambda$ 的辐射, 作用因子 $\mathrm{b}$ 必需小于等于 $\gamma \lambda$ ); 同时, 我们还必须 考虑束晕 (Beam halo) 的存在, 作用因子不能太小以至于束晕和辐射屏的相互 作用影响 ODR 的测量, 甚至产生束流损失。基于束晕模拟计算的结果[75], 在 多圈加速条件下束晕分布在 6 倍标准偏差外可以被忽略不计。从前面的 OTR 测 量我们知道束流坚直束斑 $\sigma \mathrm{y} 150 \mathrm{um}$, 因此我们选择的作用因子为 $1 \mathrm{~mm}$, 这是 
ODR 测量所用到的默认作用因子, 下文中如有变动会有特别说明。另外, 我们 还需要把束流状态从 OTR 测量的调机模式变动到连续模式, 已保证会有足够的 辐射产生。通过实验测量, 我们发现 $5.4 \mathrm{uA}$ 的连续束流就可以产生足够强的 ODR 辐射; 而在更高的电流强度下, 必需在光路中使用中性滤波片, 否则就会出现过 饱和的情况。下面就是第一次测量中 $5.4 \mathrm{uA}$ 束流下的 ODR 辐射强度分布。

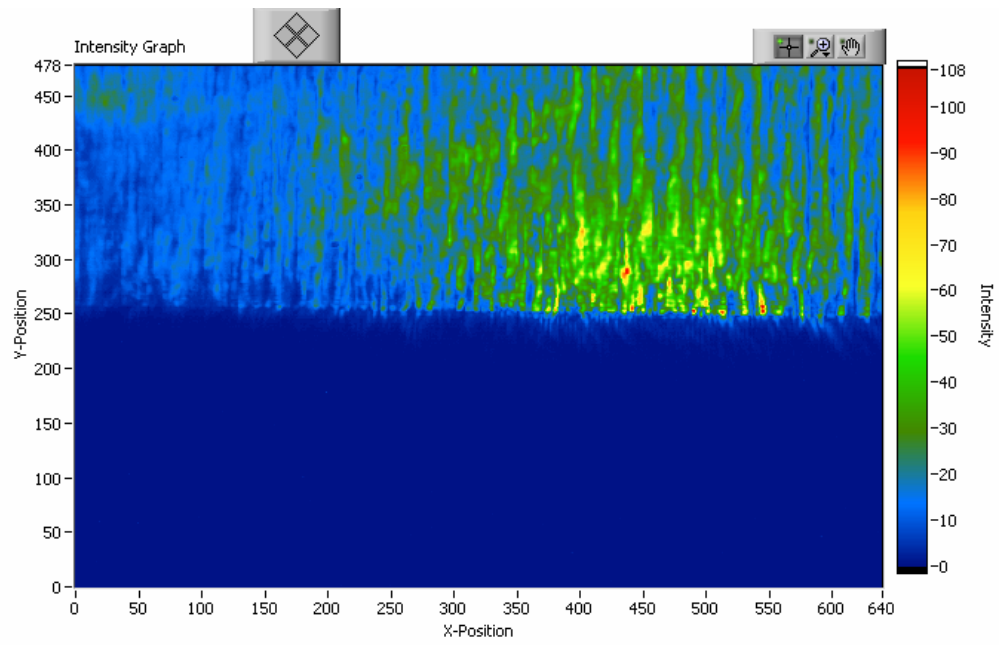

图 4.14 手工抛光铝靶 ODR 测量图

第一次测量的结果暴露了一些问题, 其中最重要的就是手工抛光的ODR屏的 镜面特性不佳。如图 4.14 所示, 辐射屏上出现的明暗相间的条纹和辐射分布的 不平滑都是屏的表面太粗糙所造成的。第二次使用镀铝硅片来代替就是为了解决 这个问题。下面给出了使用镀铝硅片的测量结果, 测量条件是 $10 \mathrm{uA}$ 连续束流、 $1 \mathrm{~mm}$ 作用因子。

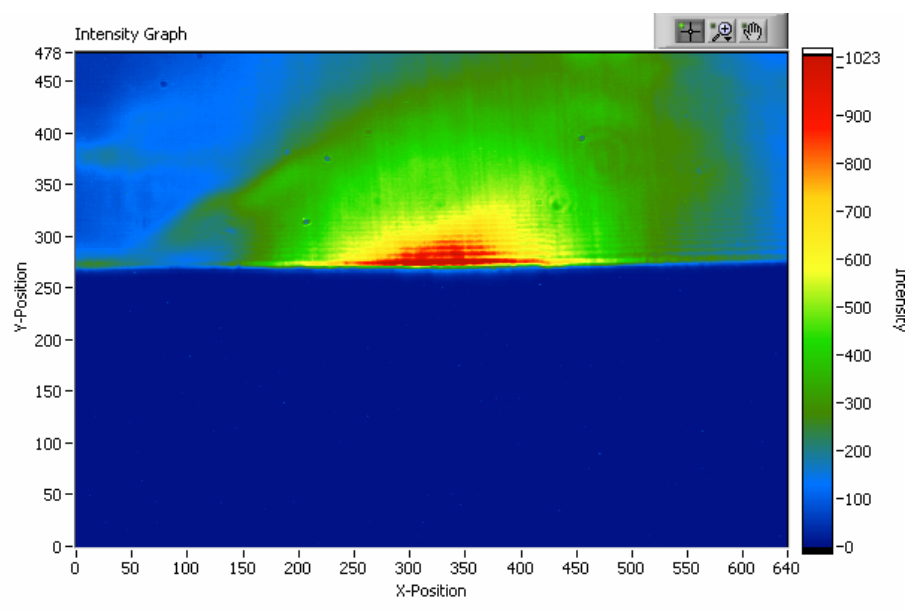

图 4.15 镀铝硅片 ODR 测量图

很明显, 辐射屏的更换大大地提高了测量的分辨率。对照模拟结果, 该测量 
结果和理论分析十分吻合。第一次测量所暴露的另外一个问题是: 束流中心并不 在视场中心, 而且视场大小不够容下ODR辐射的全分布。在对光学成像系统的 适当调节后, 我们取得了如图 4.14 中的很好的效果。

根据第二章的模拟计算结果, 我们知道 ODR 辐射的水平极化分量呈双峰分 布、坚直极化分量呈近高斯分布, 而且坚直极化分量是水平极化分量强度的约两 倍。利用两个正交极化波片, 我们测量的结果如下

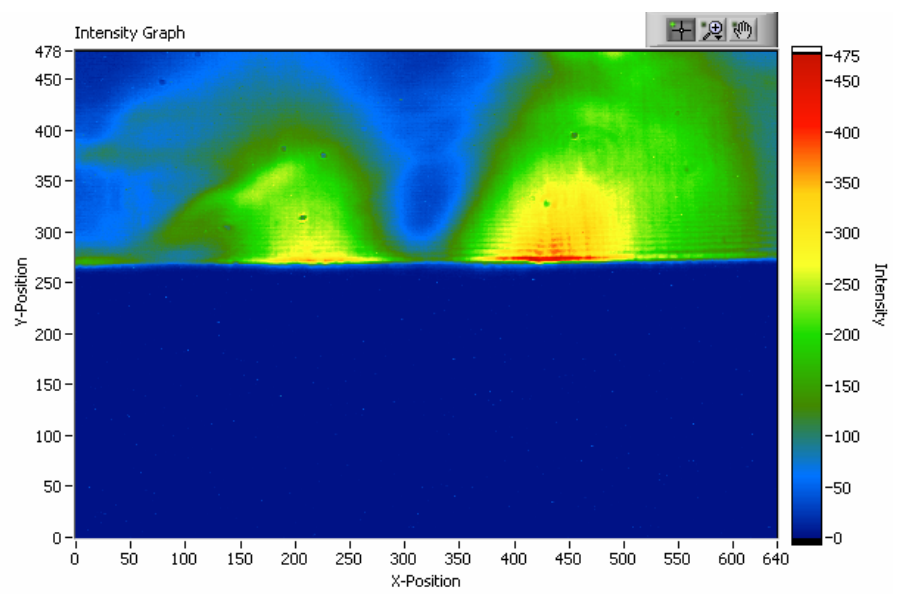

图 4.16 ODR 辐射水平分量分布

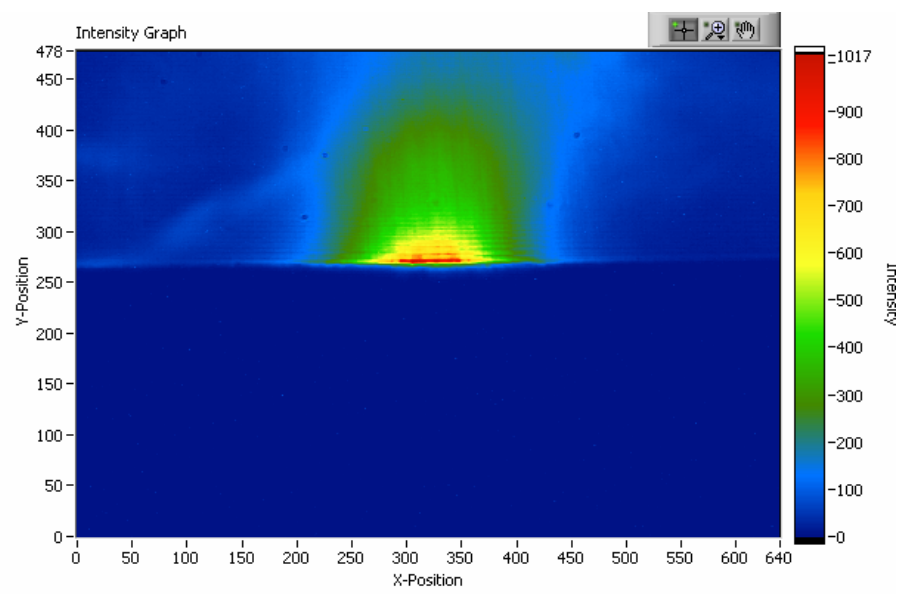

图 4. 17 ODR 辐射坚直分量分布

测量条件同样是 $10 \mathrm{uA}$ 连续束流、 $1 \mathrm{~mm}$ 作用因子。可以看到, 测量和模拟计 算的结果非常吻合。我们将在下一章中对测量结果和模拟计算作进一步的比较分 析。

我们知道, 衍射辐射产生的是连续谱。尽管因为光学元件的存在, 使得最终 在 $\mathrm{CCD}$ 相机上成像的是以可见光为中心的有限宽谱带分布。我们认为对于衍射 辐射的窄带测量能消除系统的色差、提高分辨率并有可能提供一些宽带测量无法 给予的信息。鉴于此, 我们利用窄带滤波器进行了一系列的测量。下面的测量是 
在 $45 \mathrm{uA} 、 1 \mathrm{~mm}$ 作用因子的条件下进行的, 使用了 $450 / 10 \mathrm{~nm}$ 滤波器。

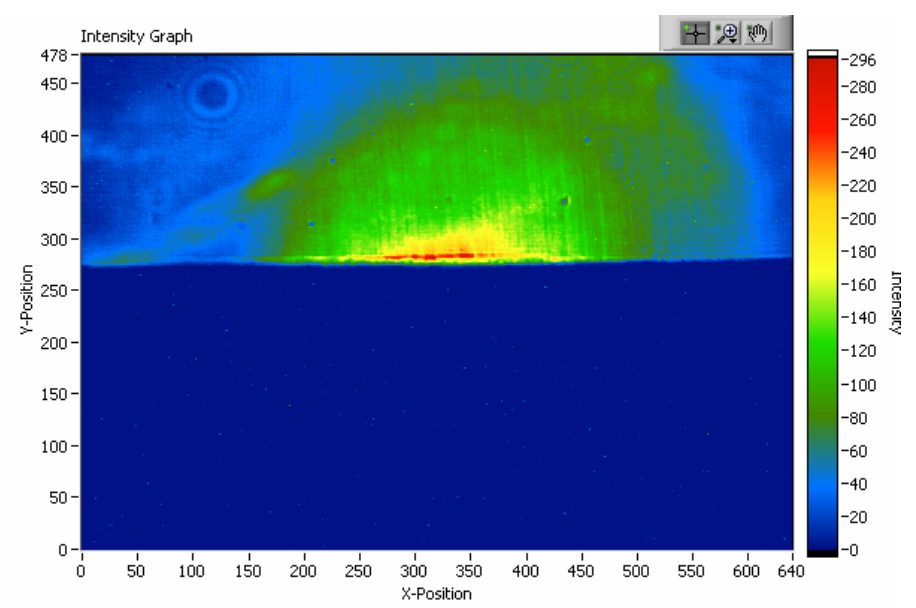

图 4.18 ODR 辐射总分布 (450/10nm 滤波器)

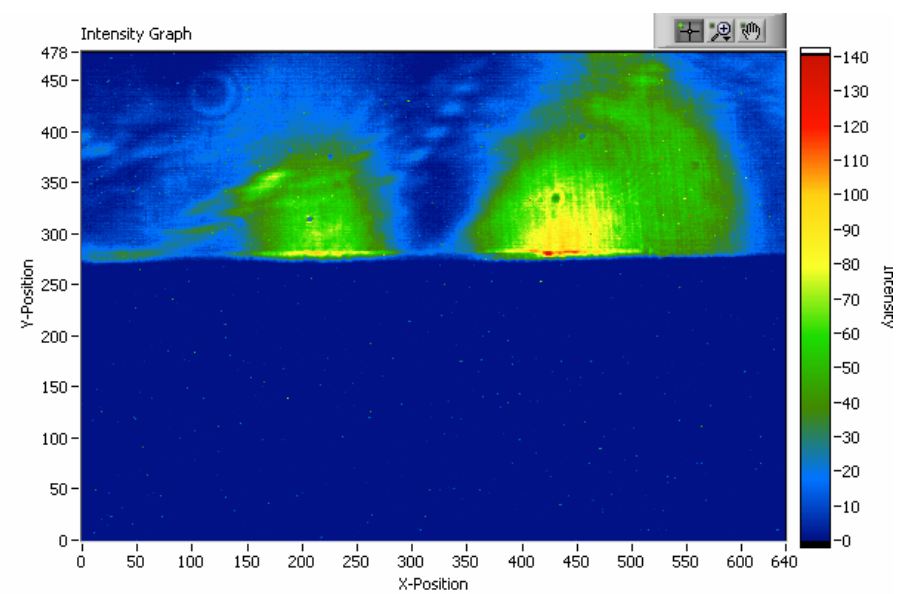

图 4. 19 ODR 辐射水平分量分布 (450/10nm 滤波器)

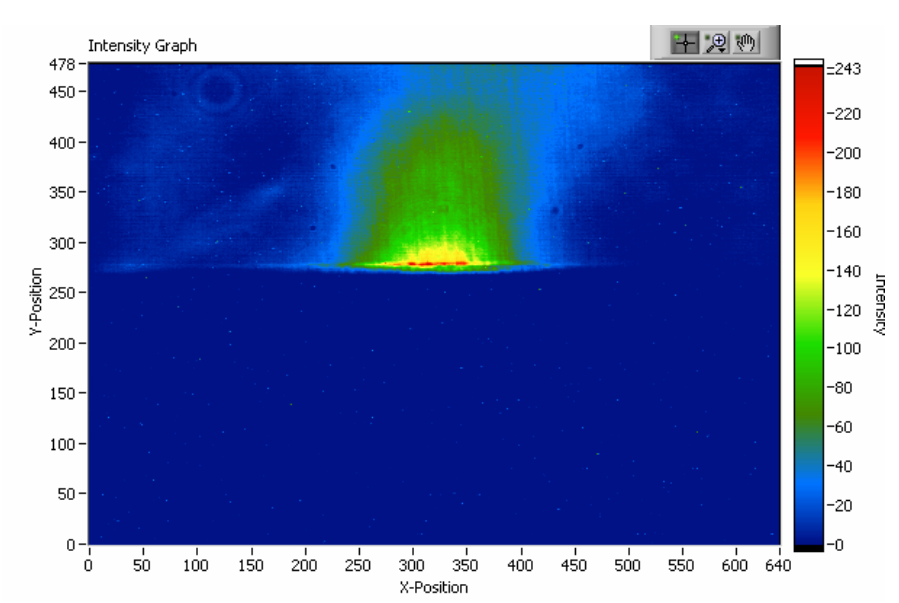

图 4.20 ODR 辐射坚直分量分布 (450/10nm 滤波器)

下面的测量是在 $45 \mathrm{uA} 、 1 \mathrm{~mm}$ 作用因子的条件下进行的, 使用了 $550 / 10 \mathrm{~nm}$ 滤 波器。 


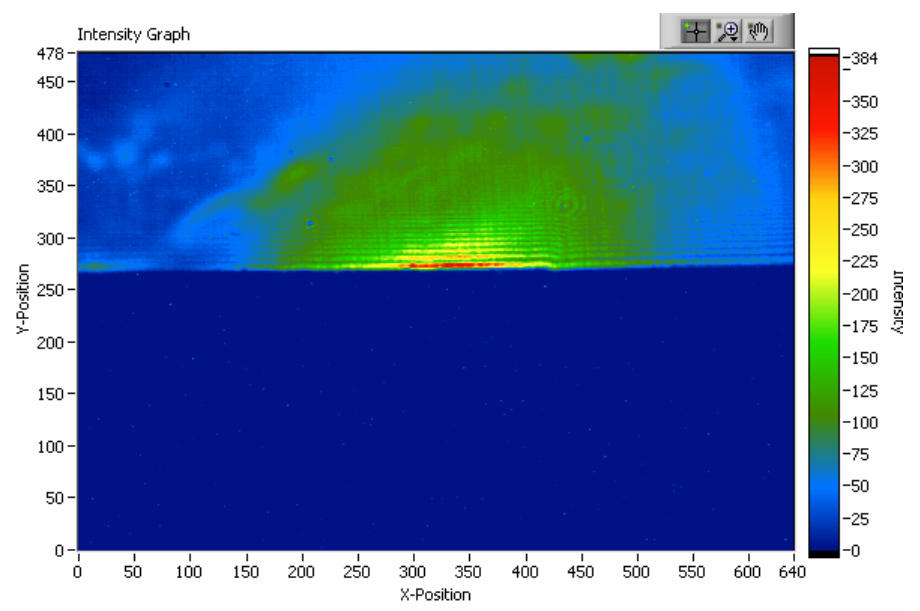

图 4.21 ODR 辐射总分布 (550/10nm 滤波器)

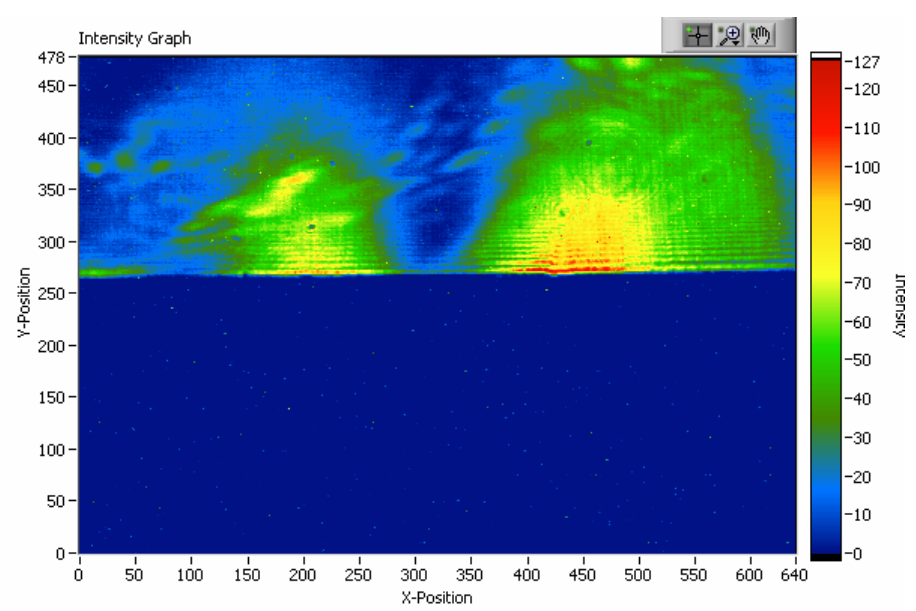

图 4. 22 ODR 辐射水平分量分布 (550/10nm 滤波器)

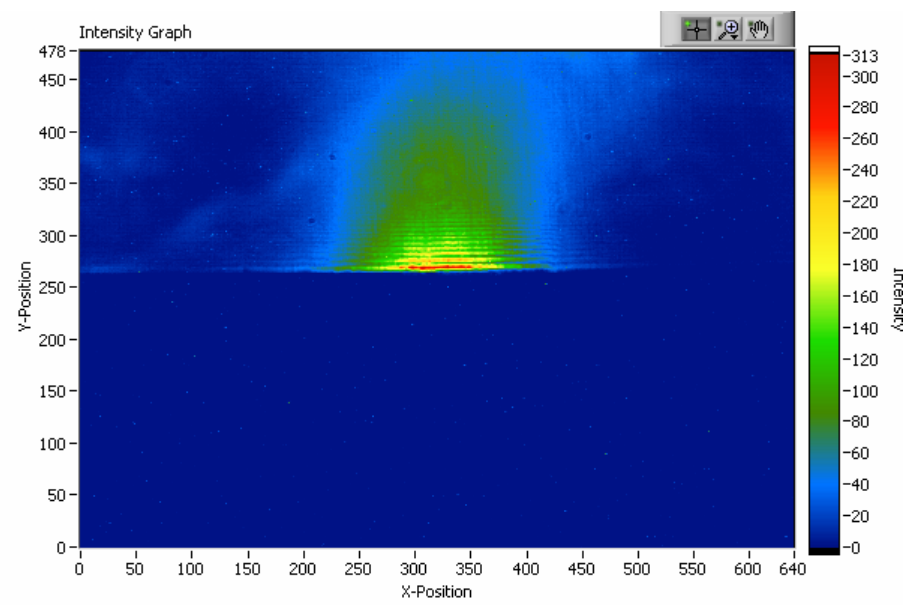

图 4. 23 ODR 辐射坚直分量分布 (550/10nm 滤波器)

下面的测量是在 $45 \mathrm{uA} 、 1 \mathrm{~mm}$ 作用因子的条件下进行的, 使用了 $650 / 10 \mathrm{~nm}$ 滤 波器。 


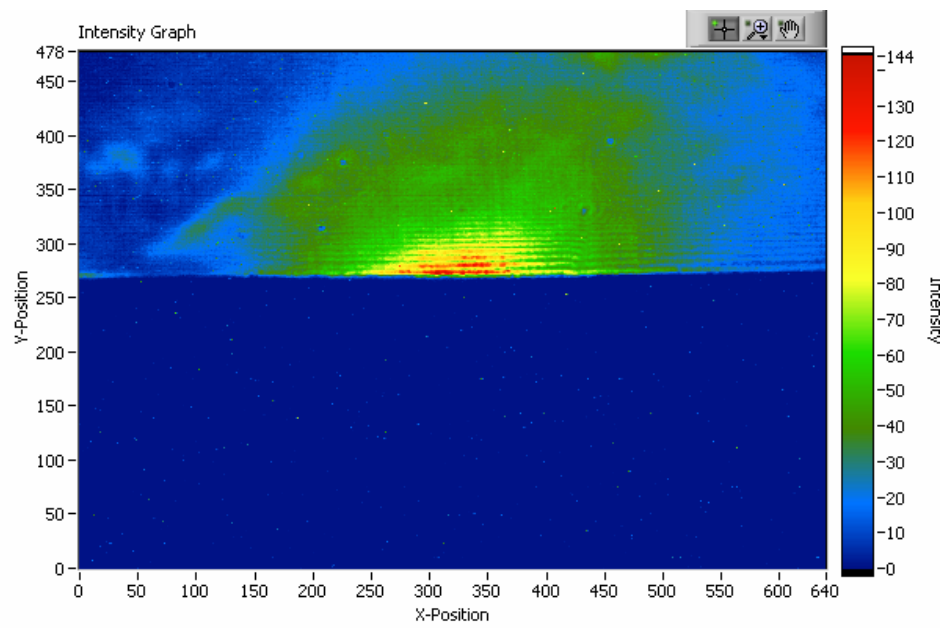

图 4.24 ODR 辐射总分布 (650/10nm 滤波器)

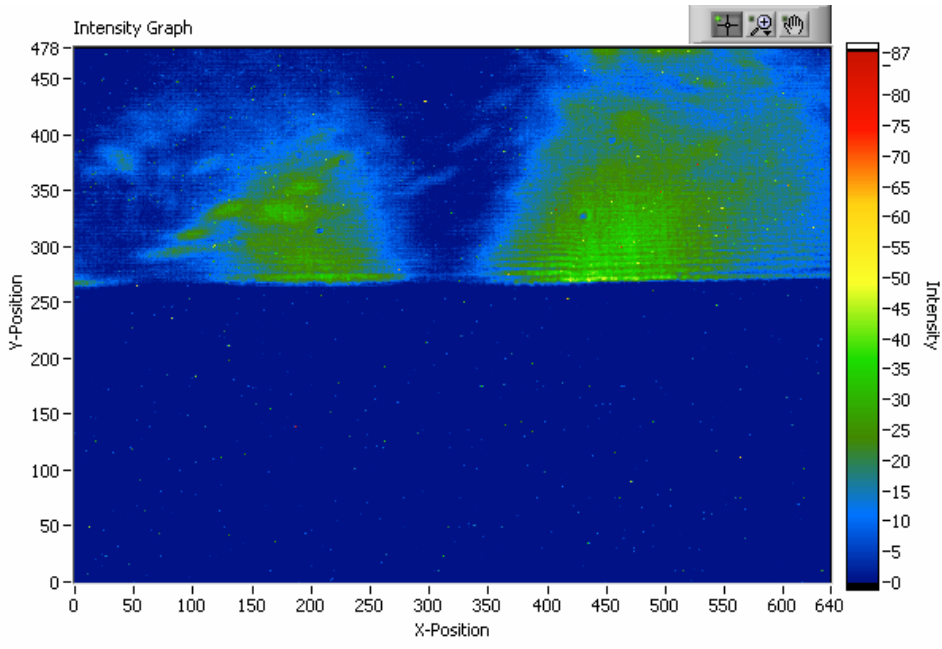

图 4.25 ODR 辐射水平分量分布 (650/10nm 滤波器)

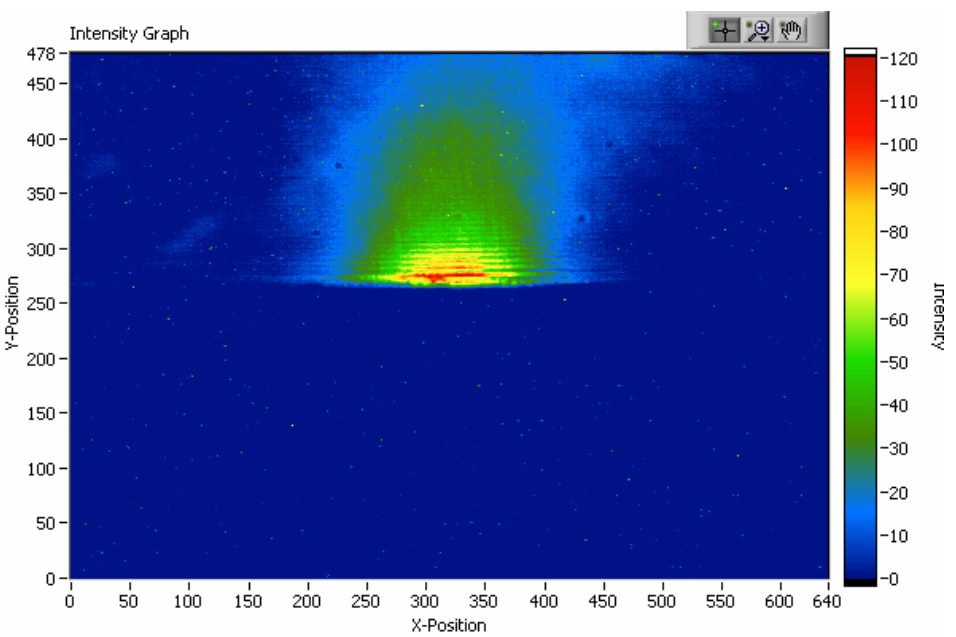

图 4.26 ODR 辐射坚直分量分布 (650/10nm 滤波器)

下面的测量是在 $45 \mathrm{uA} 、 1 \mathrm{~mm}$ 作用因子的条件下进行的, 使用了 $750 / 10 \mathrm{~nm}$ 滤 波器。 


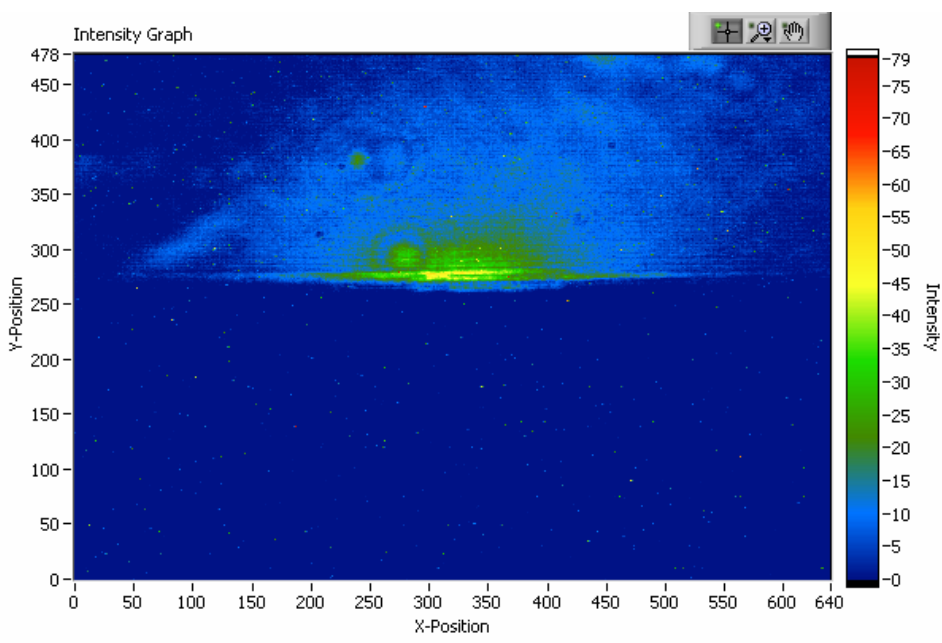

图 4.27 ODR 辐射总分布（750/10nm 滤波器）

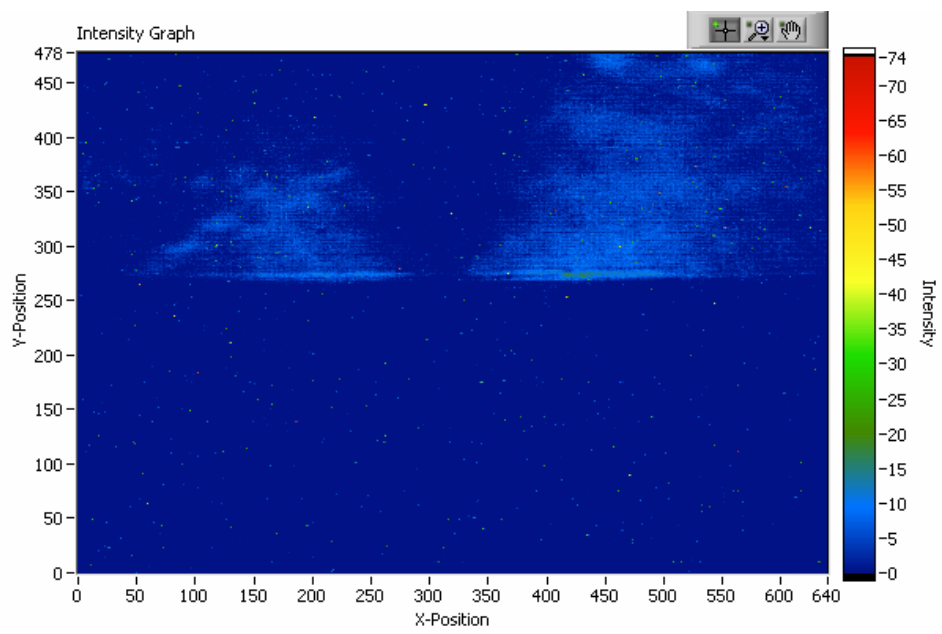

图 4.28 ODR 辐射水平分量分布 (750/10nm 滤波器)

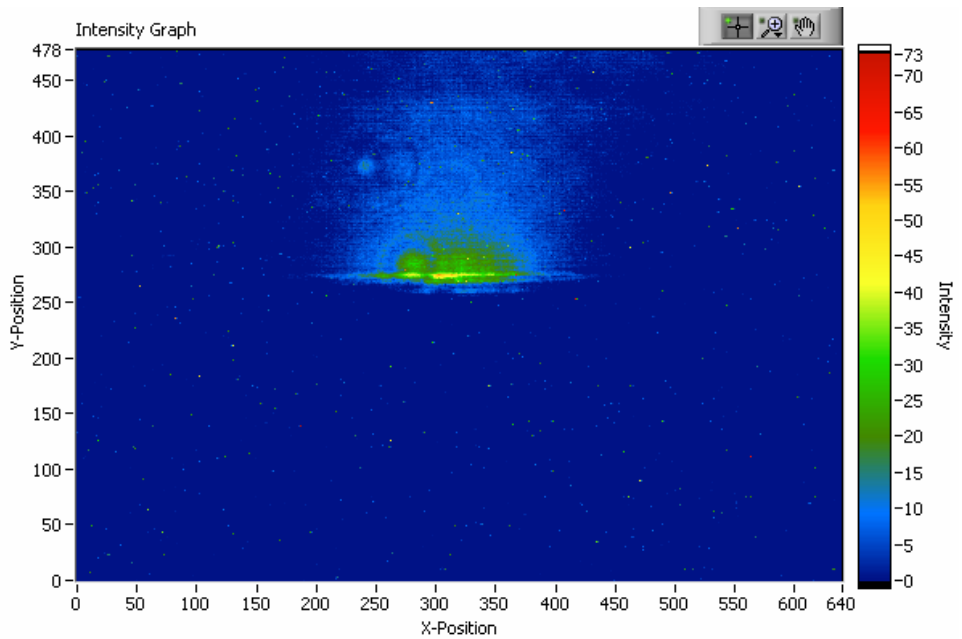

图 4.29 ODR 辐射坚直分量分布 (750/10nm 滤波器)

下面的测量是在 $45 \mathrm{uA} 、 1 \mathrm{~mm}$ 作用因子的条件下进行的, 使用了 $750 / 40 \mathrm{~nm}$ 滤 波器。 


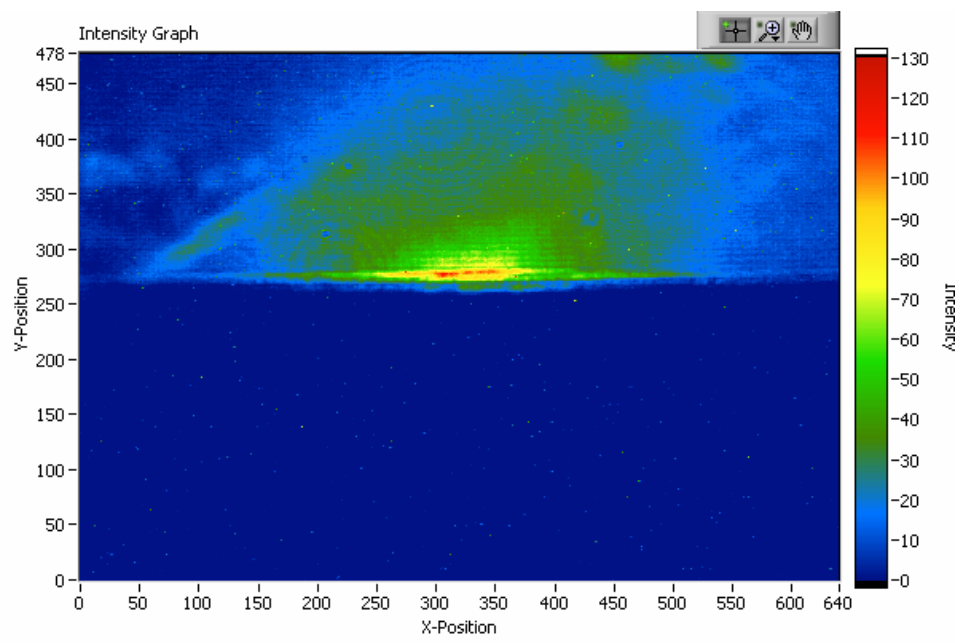

图 4.30 ODR 辐射总分布 (750/40nm 滤波器)

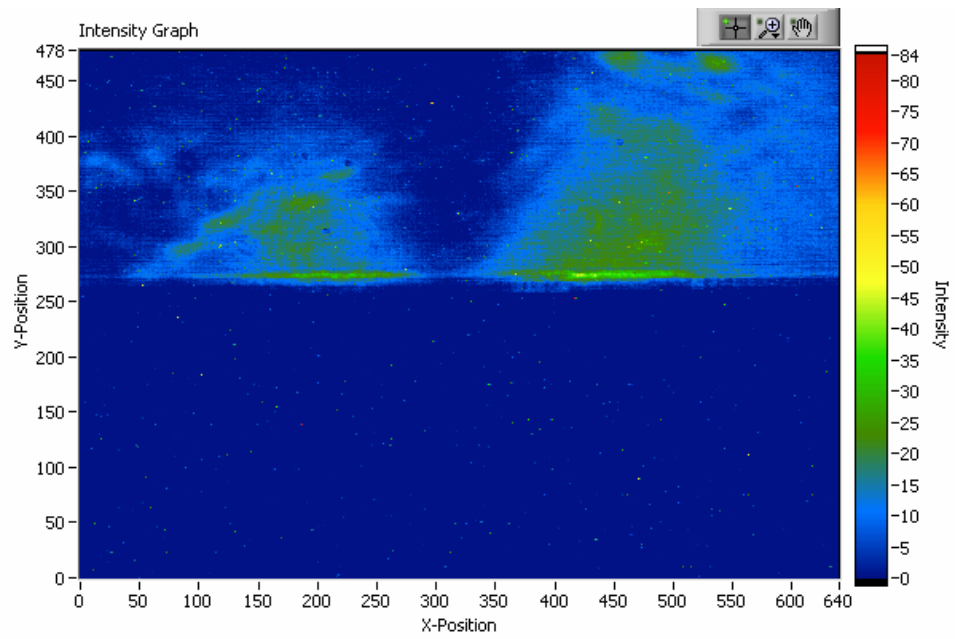

图 $4.31 \mathrm{ODR}$ 辐射水平分量分布 (750/40nm 滤波器)

下面的测量是在 $45 \mathrm{uA} 、 1 \mathrm{~mm}$ 作用因子的条件下进行的, 使用了 $500 \mathrm{~nm}$ 短通 滤波器。

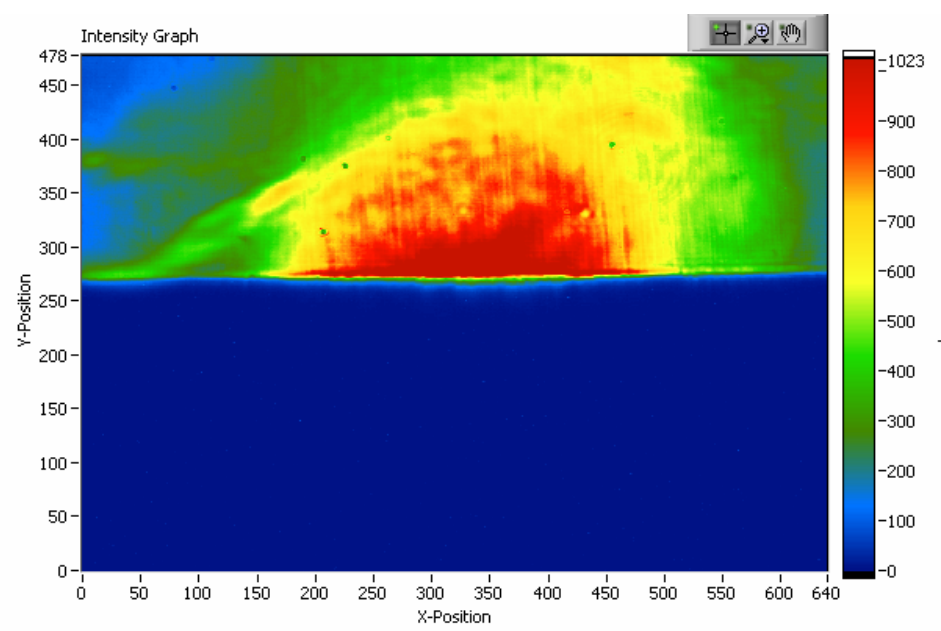

图 4.32 ODR 辐射总分布 (500nmSP 滤波器) 


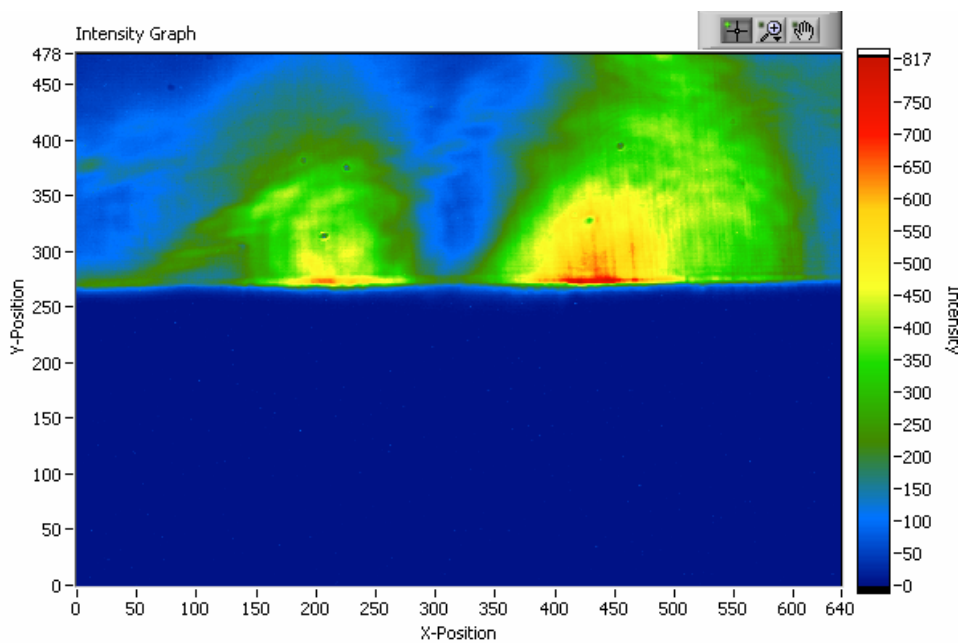

图 4. 33 ODR 辐射水平分量分布 (500nmSP 滤波器)

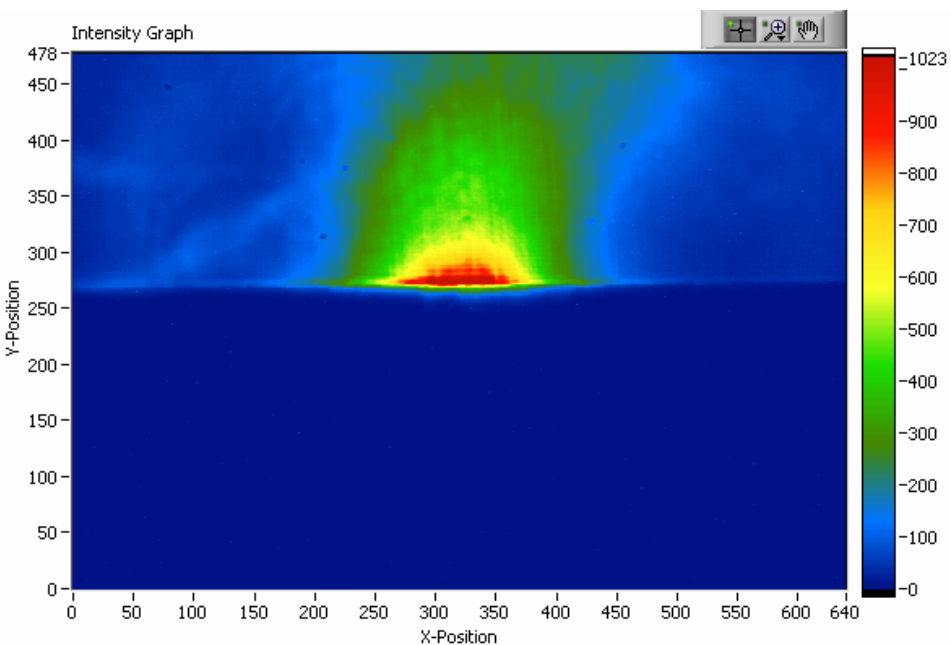

图 4. 34 ODR 辐射坚直分量分布 (500nmSP 滤波器)

下面的测量是在 $45 \mathrm{uA} 、 1 \mathrm{~mm}$ 作用因子的条件下进行的, 使用了 $500 \mathrm{~nm}$ 长通 滤波器。

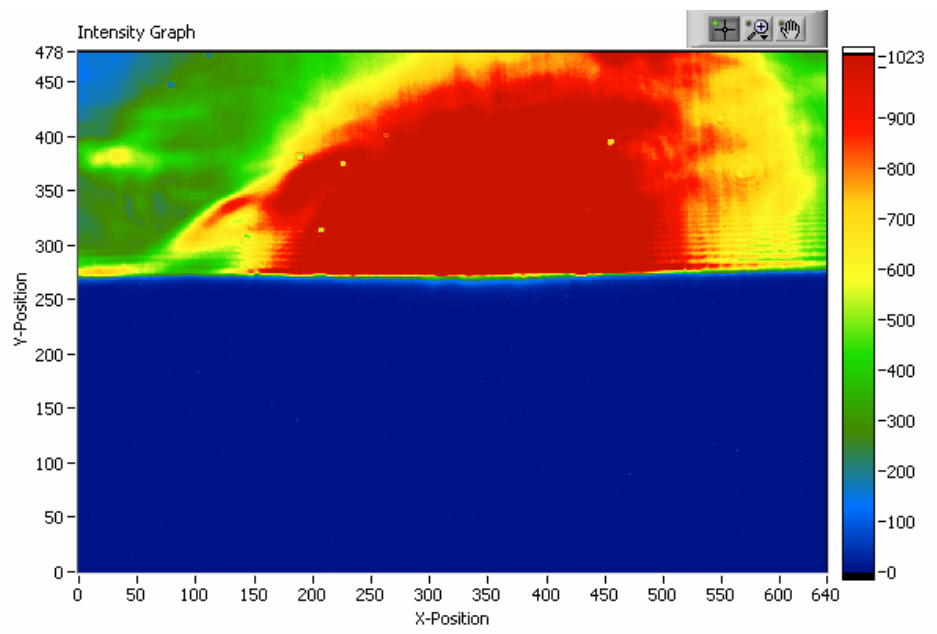

图 4.35 ODR 辐射总分布 (500nmLP 滤波器) 


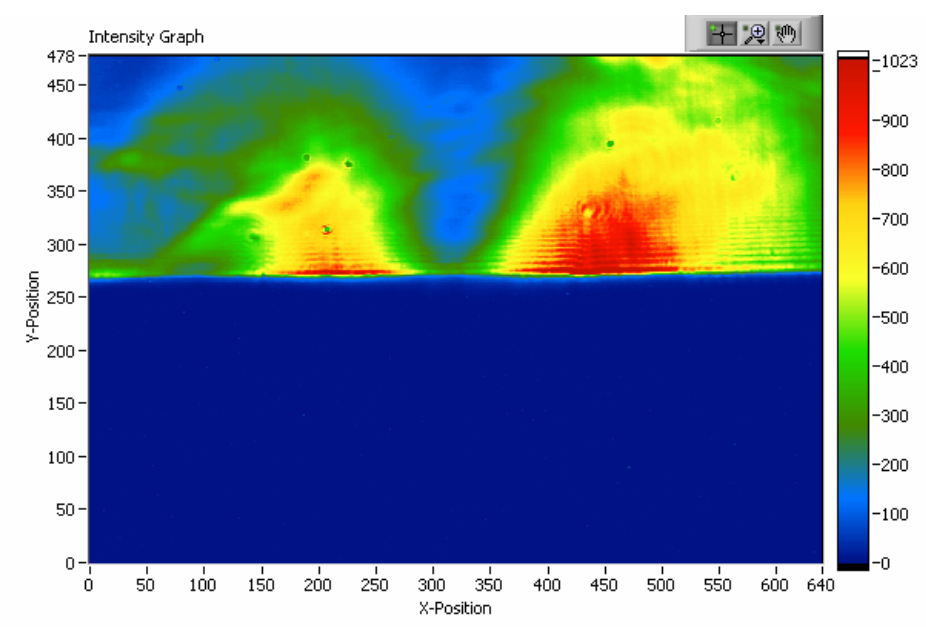

图 4.36 ODR 辐射水平分量分布 (500nmLP 滤波器)

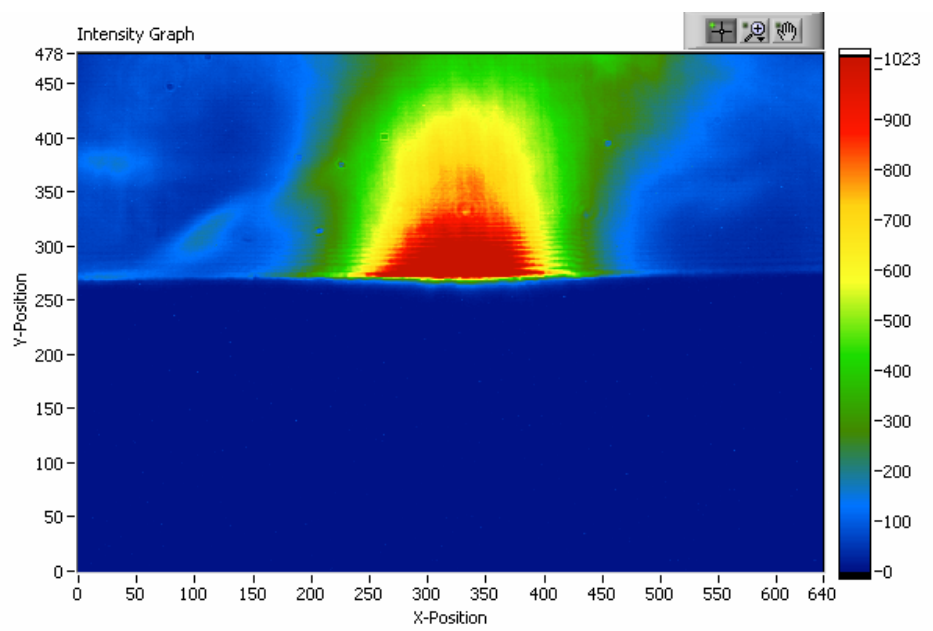

图 4.37 ODR 辐射坚直分量分布 (500nmLP 滤波器)

\section{$4.5 \mathrm{Q}$ 铁扫描测量}

为了将 ODR 辐射测量发展成为可用的束斑大小相对测量工具, 在不同的束 斑大小情况下利用多种工具的测量和比较是必需的。除了坚琴测量和 OTR 辐射 测量, 我们在相同的 $\mathrm{Q}$ 铁扫描条件下 (仅电流强度不同) 也做了 ODR 辐射测量。 所测量的数据将会在下一章进行分析和比较。

\section{6 本章小结}

本章按照测量实际进行的顺序，分别介绍了空间定标方法及前后两次方法的 改进; 坚琴测量结果及分析; 对 OTR 测量屏的改进及两次测量结果, 确定了将 镀铝硅片的 OTR 辐射测量作为有效的测量, 检验了极化波片对 OTR 辐射测量的 
作用; 介绍了如何确定衍射辐射的作用因子; 给出了衍射辐射不同极化分量的测 量、不同频带的测量及不同电流强度下的测量。 


\section{第五章 数据处理及分析}

在第四章中我们介绍了实验测量的过程, 并给出了大部分的测量结果, 也对 于衍射辐射测量有了一个初步的认识。在此基础上, 我们还需要对于测量的原始 数据进行必要的计算和分析, 从中提炼我们所需要的信息, 并同时在和理论的对 比下解释测量中所遇到的问题。首先在第一节我们会介绍所用到的处理软件; 然 后在第二节展示所用到的处理方法; 在最后一节，我们将会给出对于整章以及全 篇最重要的分析处理的结果。

\section{1 处理软件}

在数据分析处理中, 我们主要用到的软件是 LabView 和 MathCAD。图形用 户界面的 LabView 主要的作用是展示辐射强度分布图像、减除本底并可选择作 相应的高斯拟合计算。其用户界面如下所示
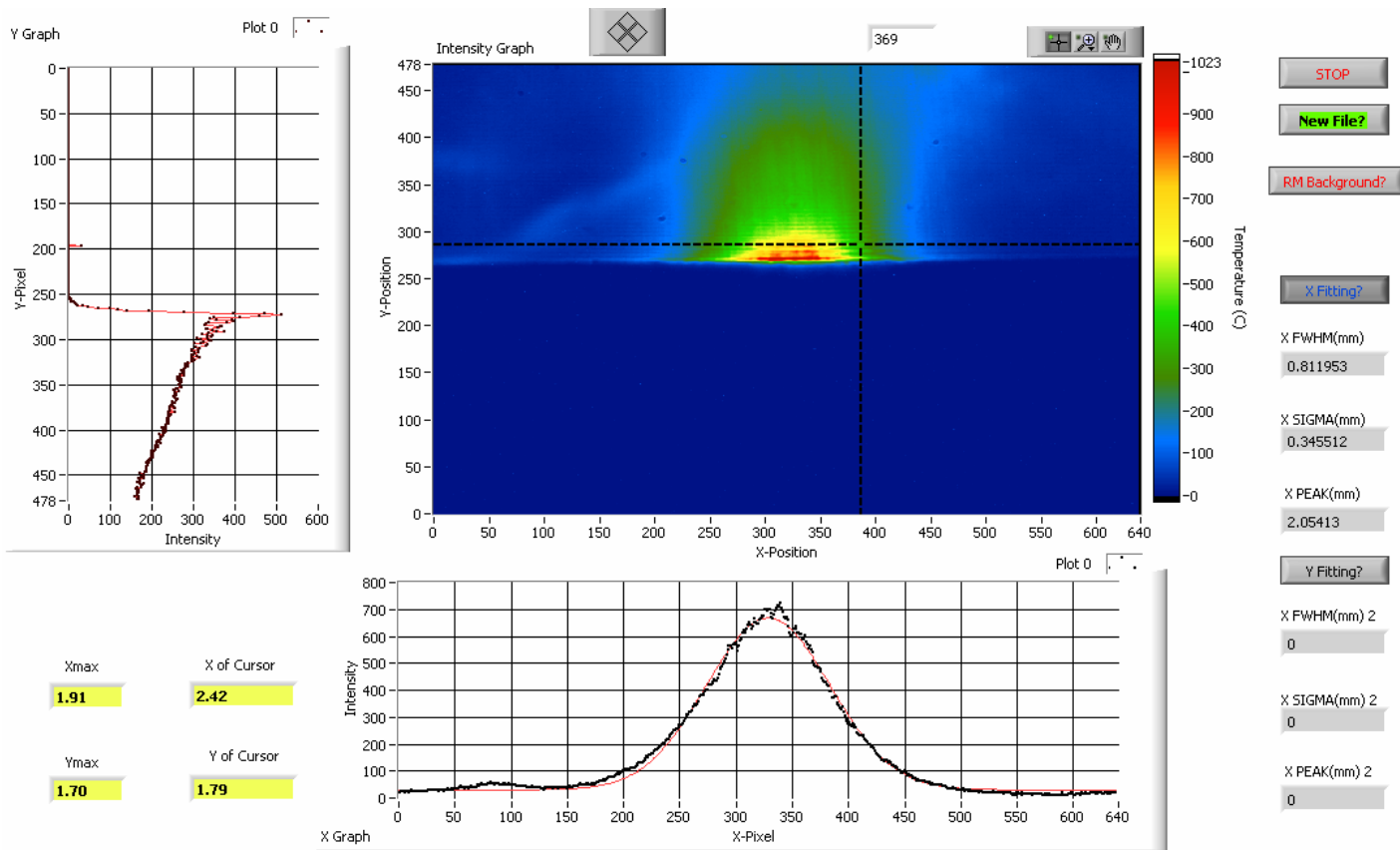

0

$x \operatorname{SIGMA}(\mathrm{mm}) 2$

$X$ PEAK(mm) 2

图 5.1 LabView 处理程序界面

MathCAD 是一款功能强大、简洁方便的科学计算软件。在本论文的工作中 主要被利用进行更复杂的模拟计算。论文中大部分的计算都是使用 MathCAD 完 成的。 


\section{2 处理方法}

对于坚琴测量、OTR 测量和 ODR 测量我们分别采用了不同的处理软件和方 法, 但简而言之所有的处理都是基于对测量分布的高斯拟合。下面对它们的处理 方法一一介绍。

Arne Freybergerger 为坚琴测量编写了一个用户界面的程序, 所使用的编程语 言是 Perl[76]。该程序能够显示峰的分布、给出峰的高斯模拟结果、测量噪声和 测量时间等信息。

对于 OTR 测量, 我们从给出的测量图像能清晰地看到其二维分布。但通常 我们更关注其沿 $\mathrm{x}, \mathrm{y}$ 的一维分布, 从二维到一维的办法就是对二维分布作投影。 如下图, 我们选取了水平方向光标之间的部分作水平投影得到水平方向上的一维 分布; 选取坚直光标之间的部分作坚直投影则得到坚直方向的一维分布。然后对 两个方向的投影进行高斯拟合并给出其标准偏差。

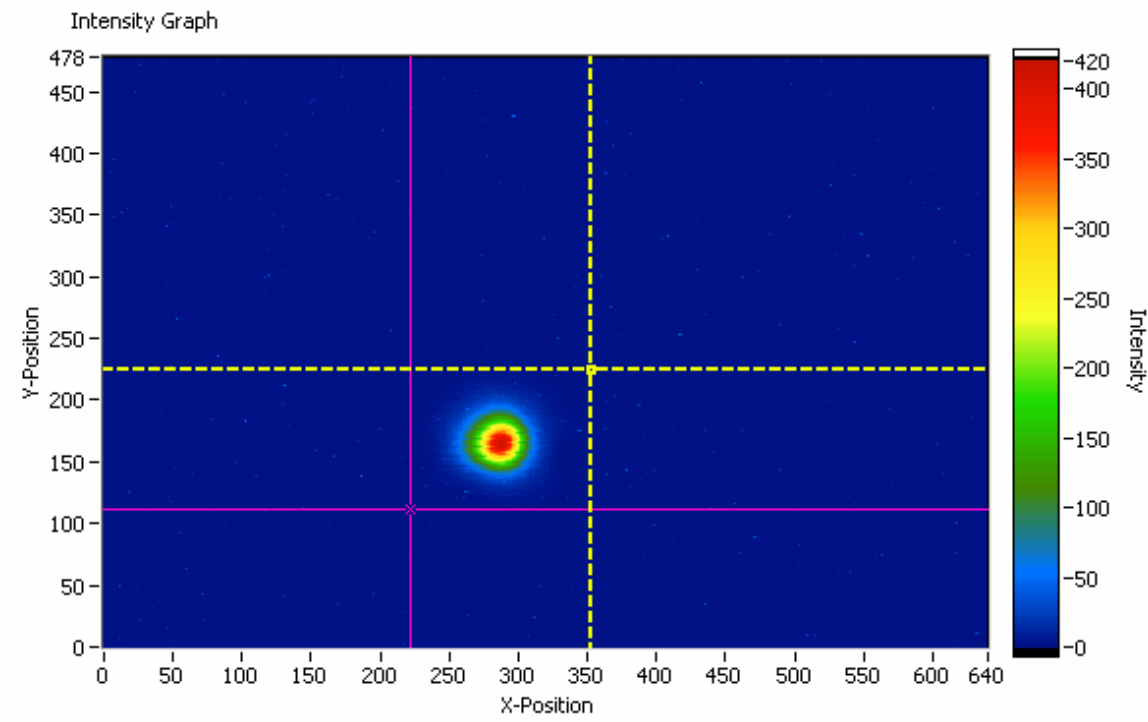

图 5.2 OTR 图像分析兴趣区（光标间的小方块）

光标范围的选择需要包含 OTR 图像的整个分布, 但不能太大以至于一些无 关区域的本底信号影响了 OTR 强度分布。另外，在束流位置不变的条件下尽量 选用相同的兴趣区域。

与 OTR 测量处理不同，ODR 测量在水平和坚直方向不能采用相同的拟合方 式，而且投影范围的选取涉及到更多复杂的因素。尽管我们知道 ODR 辐射在坚 直方向是近似指数衰减的分布, 但衰减参数和电子束两个方向束斑大小并没有直 
接明确的关系, 所以我们无法从辐射坚直分布获取有用的信息, 因此下面的讨论 都是针对 ODR 辐射水平分布的分析处理。如下图所示, 我们选取了从像素 275 到像素 300 之间的区域。一方面, 不光滑的底边上的辐射对于分析处理会有影响, 而且如前所述, 图像上底边并不成一条直线, 我们选取的起始位置要尽量避免这 些因素的影响; 另外, 在图像左上出现了其他辐射的干扰, 那么选择的终止位置 要尽量排除其他辐射的作用。

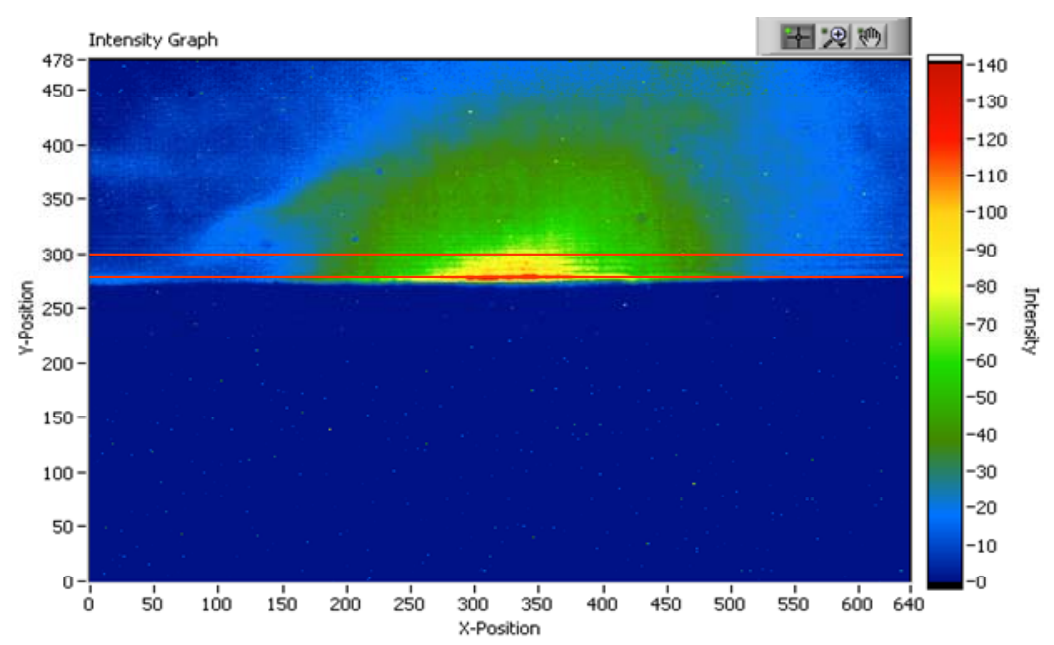

图 5.3 ODR 图像分析兴趣区

下面则是利用 MathCAD 对该区域的水平投影进行高斯拟合的结果

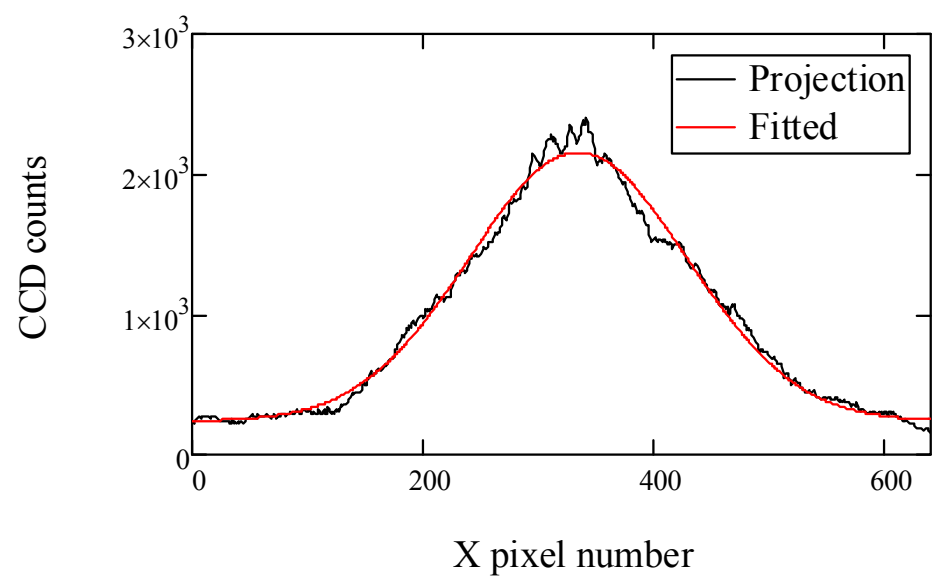

图 5.4 ODR 投影强度分布及拟合曲线

在下文中, 所有 ODR 水平图像尺寸均表示使用上面介绍的办法得到的高斯 拟合的标准偏差, 简写为 ODR image size。ODR/T 表示对总强度拟合的结果, ODR/V 表示对坚直极化分量拟合的结果。 


\section{3 分析和处理}

这一部分将要包含的内容有: 测量和理论计算的比较, 找到分歧并给出适当 的解释; 比较坚琴测量和 OTR 测量, 确定一个束斑大小的绝对测量手段; 最后 分析 ODR 测量的数据, 探索如何利用其测量束斑大小, 讨论其使适用范围。

\section{3.1 边缘辐射的贡献}

如果观察仔细, 我们会发现 ODR 辐射测量中还有其他 “不明物” 的干扰。 为了方便，下面给出几张典型的测量图片作为对比分析的对象。

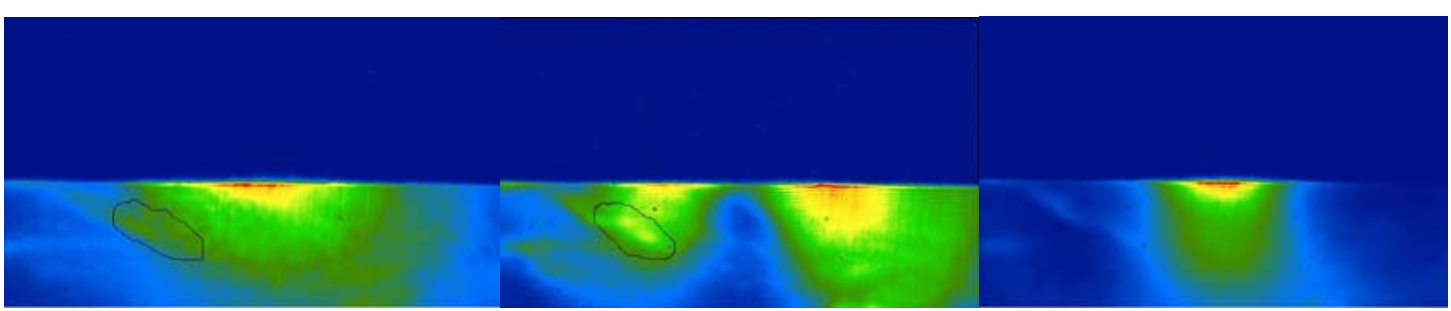

(a)

(b)

(c)

图 5.5 ODR 测量图像中的辐射干扰: a. 总强度分布中不明显的条纹;

b. 水平分量中清晰的条纹;

c. 坚直分量中条纹几乎消失

尽管在(a)中还不太明显, 我们在(b)中则可以清晰地看到它的存在。如果追索 到第一次测量, 我们会发现不明辐射的作用更加显著。从手工抛光的铝片到镀铝 硅片的改进, 不光提高了辐射屏的镜面特性, 导致 ODR 辐射及不明辐射的同时 增长; 同时也改善了辐射屏的辐射特性, 致使相同条件下 ODR 辐射在第二次测 量中比第一次中要强。所以, 第二次测量中不明辐射的作用不如之前的测量中显 著。

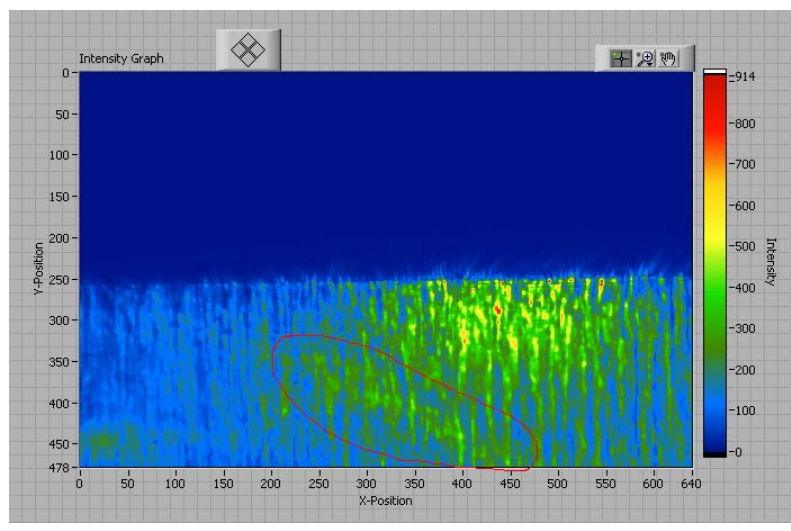

图 5.6 铝片辐射靶上的辐射干扰 
通过比较总强度、水平分量和坚直分量分布中不明辐射的强弱, 我们可以判 断的是: 该辐射可能是水平极化或者水平极化占主要。该极化特性和我们熟悉的 同步辐射吻合, 因此, 我的同事认为它就是来自于 ODR 装置上游的二极铁的同 步辐射。起初, 我并不反对这种解释, 但我认为基于极化特性上的判断并不够证 据确丵, 因此, 我进行了一些计算准备作为更有力的证据。在介绍这些计算之前 我们先看看同步辐射的一些性质。

同步辐射的 $\sigma$ 极化（极化方向沿同步辐射平面, 在我们的情况下就是水平极 化）强度分布可以表示为[77]:

$$
I_{\|}(\theta)=\frac{e^{2}}{3 \pi^{2} c}\left(\frac{\omega \rho}{c}\right)^{2}\left(\gamma^{-2}+\theta^{2}\right)^{2} K_{2 / 3}(\xi)^{2}
$$

类似地, 同步辐射的 $\pi$ 极化 (极化方向垂直于同步辐射平面, 在我们的情况下就 是坚直极化）强度分布可以表示为:

$$
I_{\perp}(\theta)=\frac{e^{2}}{3 \pi^{2} c}\left(\frac{\omega \rho}{c}\right)^{2}\left(\gamma^{-2}+\theta^{2}\right) \theta^{2} K_{1 / 3}(\xi)^{2}
$$

其中, $\xi=\frac{\omega \rho}{3 c}\left(\theta^{2}+\gamma^{-2}\right)^{3 / 2}, \theta$ 是观察方向和同步辐射平面之间的夹角, $\rho$ 是偏转 半径, $K$ 是第二类修正贝塞尔函数, $\omega$ 是同步辐射的角频率。在 $\theta=0$ 时, 同步 辐射的极化呈线性; 在夹角 $\theta$ 很大时辐射变成圆极化。

根据前面介绍的 Hall A 的光路, 我们知道 $\rho=40 \mathrm{~m}$ 。对于运行 5 圈的电子束 $\mathrm{E}=4.597 \mathrm{GeV}$, 我们考察中心频率 $\omega=2 \pi \mathrm{c} /(500 \mathrm{~nm})$ 的辐射极化状态。

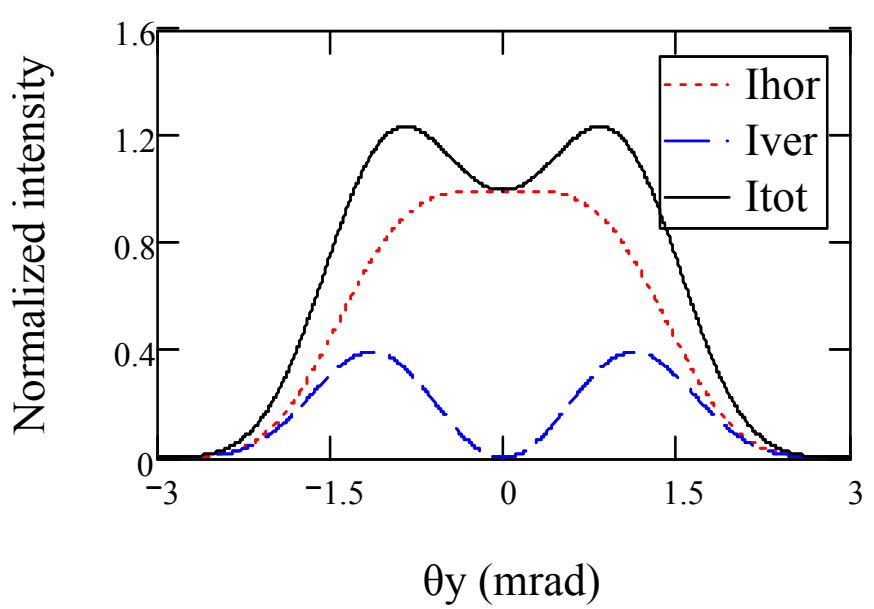

图 5.7 同步辐射坚直方向角度分布 


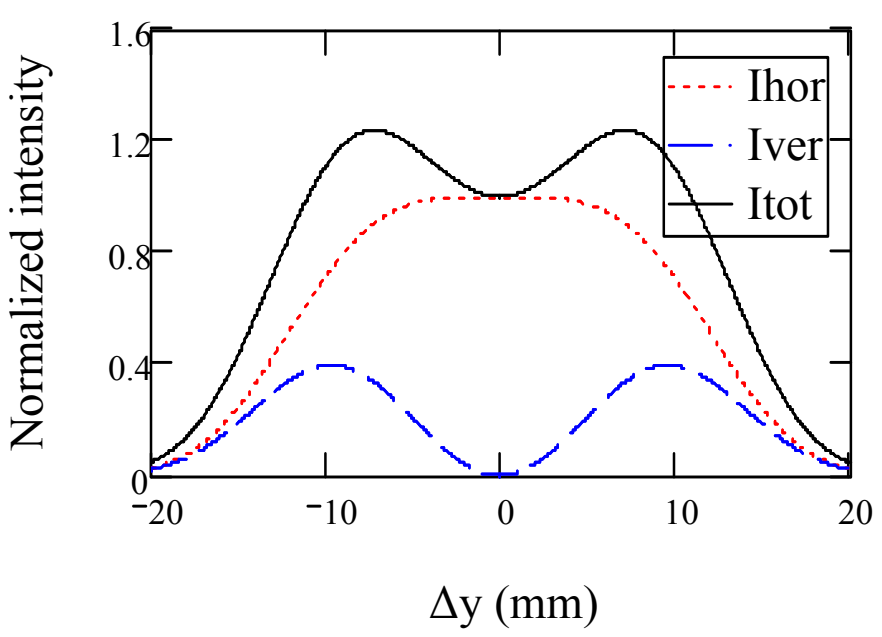

图 5.8 同步辐射在 ODR 辐射屏上坚直方向分布

其中, 图 5.8 是基于最后一个二极铁离ODR辐射屏 $8.1 \mathrm{~m}$ 而作的。很明显, 同步辐射的水平极化分量比坚直极化分量要强 2 3 倍, 尽管我们从ODR辐射测 量中无法知道二者的定量关系, 但这和观察到的二者强弱是符合的。但是从 1 可以看到, 可见光部分同步辐射的发散角约 $1 \mathrm{mrad}$, 在我们测量的情况下比辐射 中心波长的张角 $1 / \gamma$ 要大很多。经过 $8.1 \mathrm{~m}$ 自由空间的传播后, 该辐射条纹在坚 直方向应该会发散至 $20 \mathrm{~mm}$ 左右。但是, 我们在测量中所看到的辐射是约 $0.2 \times$ $1 \mathrm{~mm}^{2}$ 的条纹。以上的推理导致我对于该条纹来源于同步辐射的解释产生了怀疑。

另外, 我们也可以从该条纹的强度出发考虑以上论点的真实性。根据 ODR 辐射的解析表达式, 我们可以推理最强的衍射辐射微分强度可以表示为:

$$
\frac{d I}{d \omega}=\frac{1}{\pi^{2}} \frac{e^{2}}{c}\left(\frac{\omega}{\gamma c}\right)^{2} K_{1}\left(\frac{\omega}{\gamma c} b\right)^{2}
$$

对于同步辐射, $\theta=0$ 时微分强度最强。考虑可见光范围的辐射（波长远长于特 征波长), 微分强度可以表示为[78]

$$
\frac{d I}{d \omega}=\frac{e^{2}}{c}\left(\frac{\omega \rho}{c}\right)^{1 / 3}
$$

利用上面公式, 我计算了波长为 $500 \mathrm{~nm}$ 的同步辐射微分强度和作用因子 $\mathrm{b}=1 \mathrm{~mm}$ 、 波长同样为 $500 \mathrm{~nm}$ 的衍射辐射的微分强度。结果是, 衍射辐射的强度是同步辐 射强度的 18.7 倍, 甚至 $b=1.2 \mathrm{~mm}$ 时衍射辐射还是同步辐射的 7.8 倍。上面是在 特定的波长强度的计算, 如果在计算中对两个表达式都作可见光波长范围内的积 分, 衍射辐射将会更强, 这从表达式中强度与辐射频率的关系可以看出。所以, 
相同电子束在我们的实验条件下是无法产生和衍射辐射相同强度的同步辐射的。 这是我反对该条纹来自于同步辐射的第二个原因。

推翻了同步辐射说之后, 我自然地开始考虑边缘辐射的可能性[31]。边缘辐 射是带电粒子束进入或离开二级铁时在二极铁边缘场的作用下的辐射。下面是一 些边缘辐射性质的总结[79-83]:

1. 对于同步辐射, 在辐射特征波长 $4 \pi \rho / 3 \gamma^{3}$ 两侧的辐射功率相等。和同步辐射一 样, 边缘辐射来自于垂直于运动方向的加速度, 我们可以把边缘辐射看作是渐弱 或渐强二极场作用下的同步辐射。如果是电子束离开二极铁时产生的辐射, 电子 束的弯转半径逐渐变大, 所以辐射谱的中心向着长波长移动。自然, 边缘辐射在 长波的辐射强度会强于同样条件的同步辐射。

2. 相比于同步辐射, 边缘辐射集中在沿电子径迹很小的角度范围内。

3. 在波长范围 $\left(\rho \theta^{3}, R \theta^{2}\right)$ 内, 这里 $R$ 是从二极铁边缘到观察点的距离, 边缘辐射 的辐射通量比同步辐射要强。对于我们的情况, 该波长范围是 $(71 \mathrm{~nm}, 12 \mathrm{um})$ 。

从以上的性质 (特别是第 2 条) 来看, 边缘辐射是很有可能是该条纹产生的 源头。但是定性的分析是不够充分的, 下面是我所作的一些定量的计算。

表格 5.1 SRW 模拟计算使用的束流参数

\begin{tabular}{ccc}
\hline Parameters & values & units \\
\hline $\mathrm{E}$ & 4 & $\mathrm{GeV}$ \\
$\Delta \mathrm{P} / \mathrm{P}$ & 0.1 & $\%$ \\
$\mathrm{I}$ & 100 & $\mathrm{uA}$ \\
$\varepsilon \mathrm{x}$ & 3.9 & $\mathrm{~nm}$ \\
$\varepsilon \mathrm{y}$ & 2 & $\mathrm{~nm}$ \\
$\beta \mathrm{x}$ & 35.6 & $\mathrm{~m}$ \\
$\beta \mathrm{y}$ & 20 & $\mathrm{~m}$ \\
\hline
\end{tabular}

尽管有很多针对边缘辐射的研究工作, 边缘辐射还没有一个统一的模型, 其 辐射分布的计算还是一个比较复杂的。基于 [84]中的分析方法, 边缘辐射的计算 被作为一个模块置入了同步辐射计算软件 SRW 中。依据表中的数据, 我们假设 有效边缘场长度为 $40 \mathrm{~mm}$ 的条件下计算了电子束在离开最后一块二极铁时产生 的边缘辐射。下面是二极场强的分布图: 


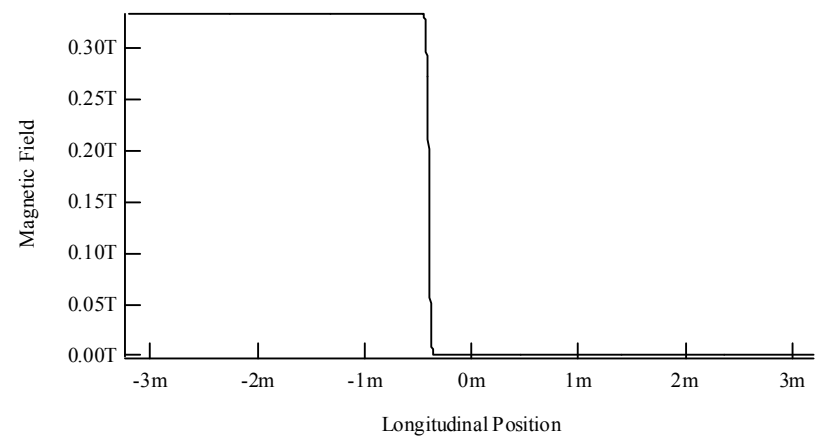

图 5.9 二极铁边缘场分布
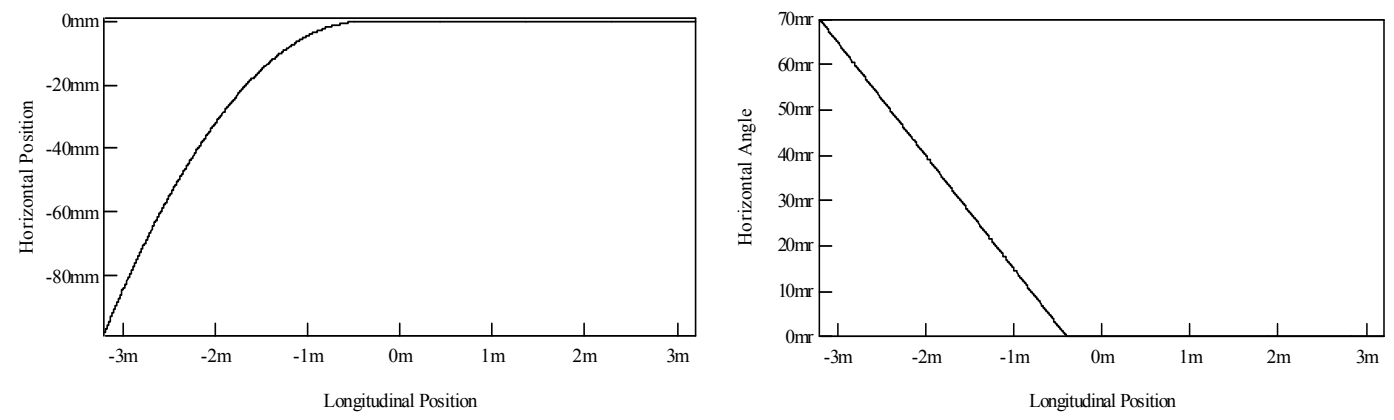

图 5.10 电子束横向位置及角度的变化

下面是边缘辐射两个极化分量的分布和二维分布图
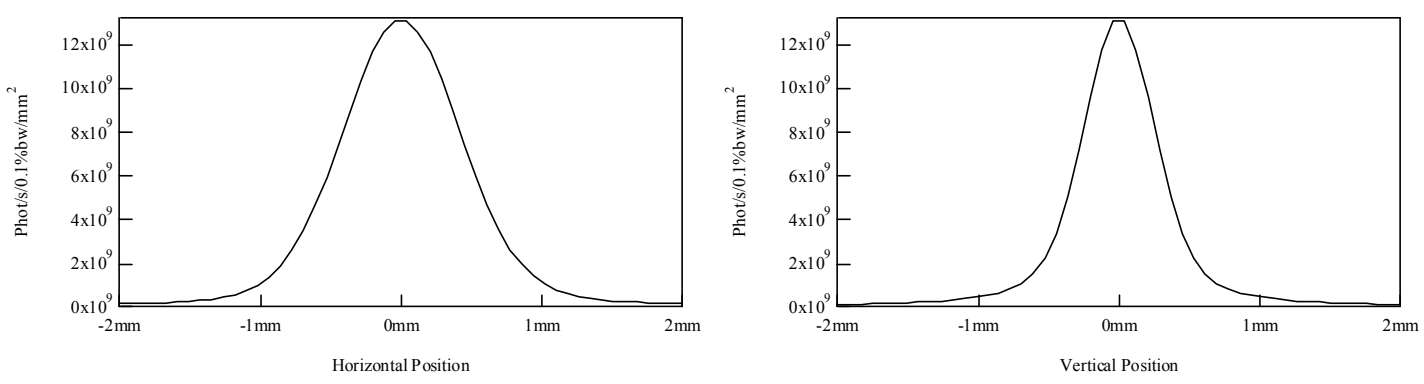

图 5.11 边缘辐射沿水平和坚直方向的分布

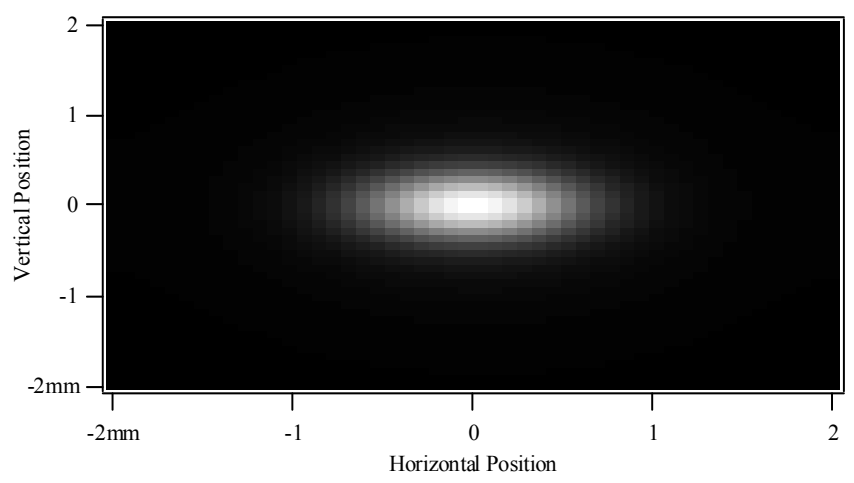

图 5.12 边缘辐射在 ODR 屏上的二维分布

上面所显示的是波长为 $500 \mathrm{~nm}$ 的边缘辐射。和我们预期的一样, $500 \mathrm{~nm}$ 的边 缘辐射通量比同等条件下的同步辐射要强。上面的计算告诉我们: 可见光附近的 
边缘辐射强度足够干扰衍射辐射; 边缘辐射被限制在很小的角度范围内, 不会像 同步辐射一样很快发散; 在观察平面上, 边缘辐射产生的条纹和我们观察到的条 纹大小一致。基于以上的分析, 我们可以确认 ODR 辐射屏上的条纹来自于最后 一块二极铁的边缘辐射。

需要补充说明的是, 我们模拟计算得到的辐射条纹和观察到的并不在一个方 向上, 原因是我们使用的是机器的设计参数, 而实际参数和设计值稍有出入。最 后一块二极铁处电子束的截面是倾斜的，并非设计的正椭圆。另外，从二极铁到 辐射屏的短暂传播并不会改变束流截面分布, 边缘辐射的分布应该和二极铁处的 电子束截面分布取向一致, 因此, 辐射屏上OTR图像也应该和该辐射条纹取向一 致。这一点在 图 5.13 中被证实, 图中是在相同的束流尺寸下做的ODR辐射和 OTR图像合集。

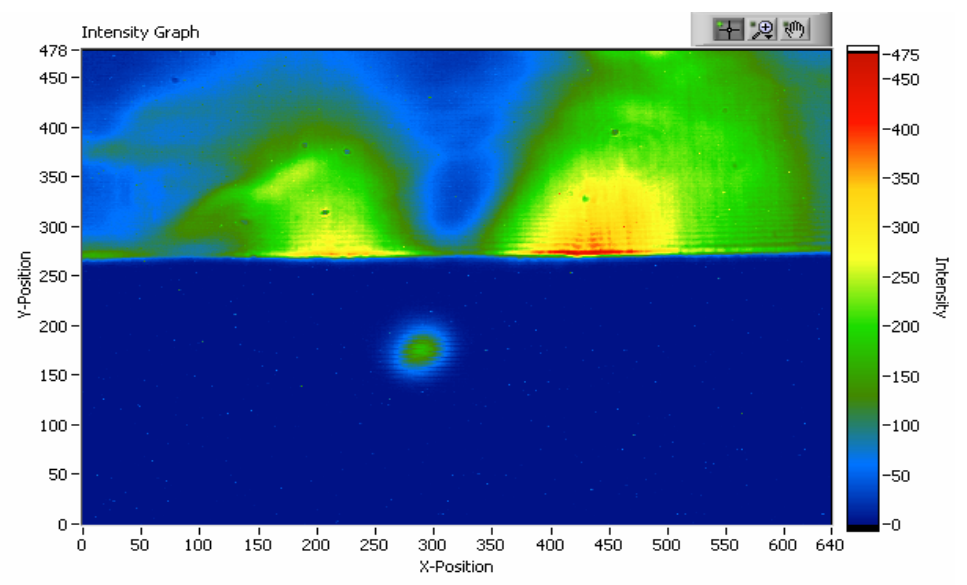

图 5.13 ODR 辐射和 OTR 辐射图像合成

\section{3.2 针对边缘辐射的提案}

在了解辐射干扰的来源后, 我们自然需要考虑如何消除边缘辐射对于 ODR 辐射测量的影响。在数据处理方法的介绍中, 我们所到了因为辐射干扰的存在而 很大地限制了处理数据的灵活性。而且, 通常 ODR 辐射测量装置位置的选择都 很难规避二极铁的存在。所以, 下面将要介绍的方案不仅对于我们的测量调高精 度有帮助，也对于今后的 ODR 辐射测量工作具有普遍的借鉴意义。

第一种提案的思想很简单, 在 ODR 辐射屏前一段距离将电子束向上偏转, 并在辐射屏之后校正回来, 进行相同的测量就需要将辐射屏向上移动以保持相同 的作用参数, 这样边缘辐射会相对辐射屏向下移动, 离开系统视场甚至整个辐射 
屏。尽管原理简单, 该方法在 CEBAF 机器上实现会有一定的困难, 因为矫正铁 产生任何的辐射和束流位置变化都会对物理实验产生影响。

第二个是一个巧妙、简单易行且有附加好处的方案。为了理解, 我们需要仔 细研究我们的成像系统。如图 5.14, 它是被简化的成像系统, $\mathrm{O} 1$ 是最后一块二 极铁处产生的边缘辐射, $\mathrm{O} 2$ 是辐射屏上的衍射辐射, I1、I2 是 $\mathrm{O} 1 、 \mathrm{O} 2$ 分别的 像。基于之前讨论, 图中光线所描述的边缘辐射的发散角相比衍射辐射要小。因 此, 在 $\mathrm{ABCD}$ 平面边缘辐射集中在了一个很小的面积上 (用 $\mathrm{BC}$ 表示), 而衍射 辐射还散布在很大的范围内 (用 $\mathrm{AD}$ 表示)。这样, 如果在该平面处放置一个小 的遮光物, 则可以消除绝大部分的边缘辐射而不会太影响衍射辐射的强度。

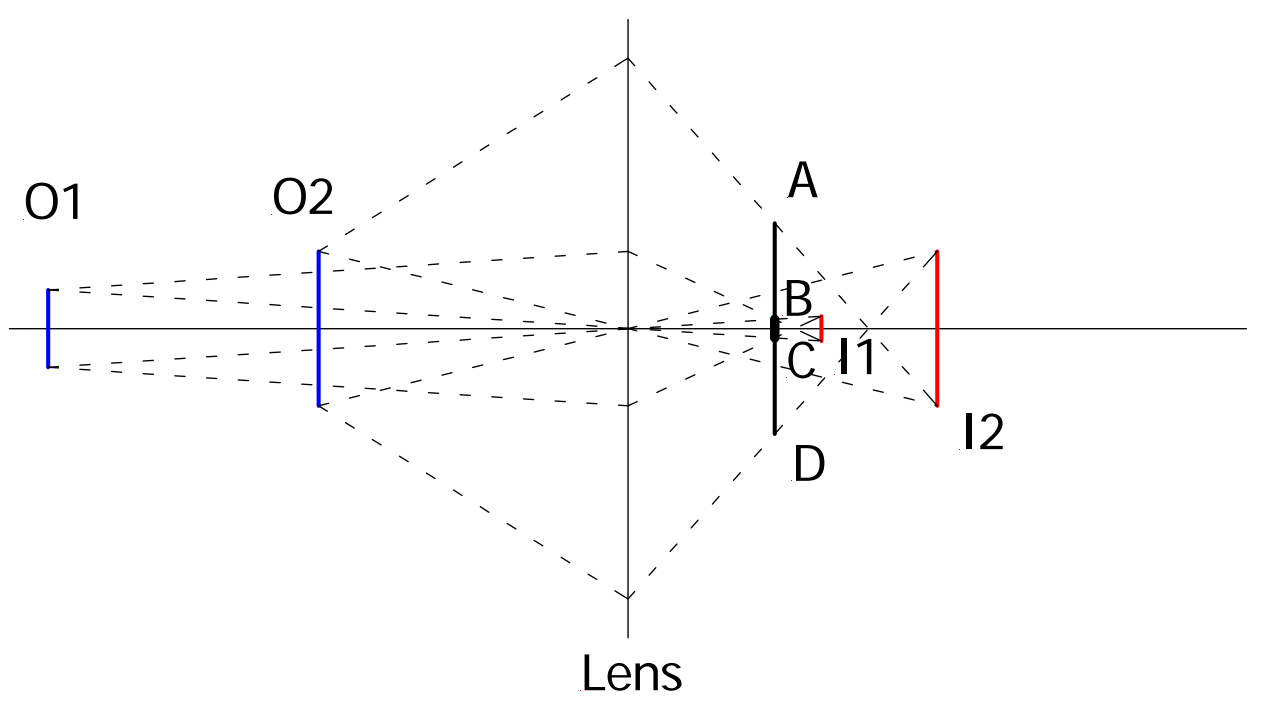

图 5.14 衍射辐射及边缘辐射成像示意图

根据在测量精度中的讨论, 在系统光轴上搁置遮光物能够压缩点发散函数从 而提高系统测量的精度。因此, 该方法因为只涉及对光路的改动而简单易行, 同 时又为我们带来高精度的附加好处。

\section{4 水平极化分量的非对称}

如果对比测量的水平极化分量和模拟计算的结果, 我们发现实际测量的分布 并不像计算的那样是对称分布。下面我们就该不对称现象进行分析和解释。

起初, 我的同事们认为是截面倾斜的电子束造成了不对称。如图 5.13, OTR 图像显示电子束截面是一个近似斜粗圆, 其右侧离辐射屏底边更近。因此, 导致 了 ODR 辐射的水平极化分布右侧的峰更强。该解释似乎很合理, 但为了得到更 
加充分和证据确丵的解释, 我进行的一些计算。

我的基本思路是, 假设电子束截面倾斜, 然后计算由其产生的 ODR 辐射水 平极化分量的分布情况。我们假设电子束截面如图 5. 15 所示倾斜 $45^{\circ}$ 角。在 $\mathrm{xy}$ 坐标系内, 束截面是一个长轴在 $\mathrm{x}$ 上短轴在 $\mathrm{y}$ 上的高斯分布的椭圆。根据坐 标转换规律, 电子束截面内相同的点在 x’y'坐标系和在 $x y$ 坐标系内的坐标转换 可以表示为[85]

$$
\left(\begin{array}{l}
x^{\prime} \\
y^{\prime}
\end{array}\right)=\left[\begin{array}{cc}
\cos \theta & -\sin \theta \\
\sin \theta & \cos \theta
\end{array}\right]\left(\begin{array}{l}
x \\
y
\end{array}\right)
$$

\section{$\mathrm{y}^{\prime}$}

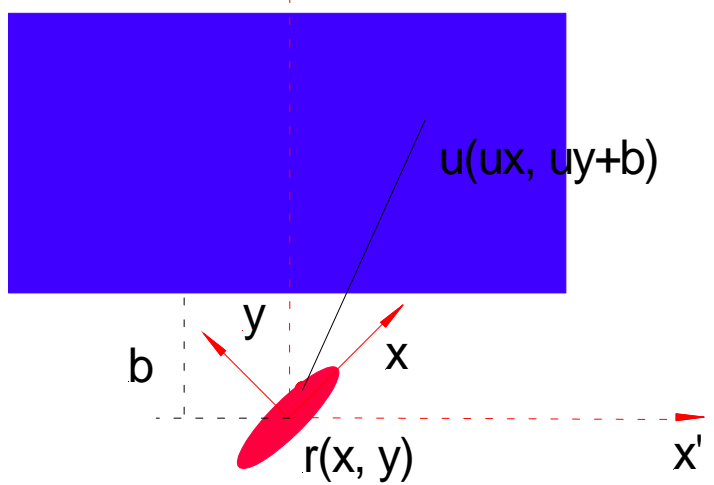

图 5.15 倾斜截面电子束衍射辐射示意图

根据我们前面介绍的 ODR 辐射的微分强度公式, 在束流倾斜的条件下, 总强度 分布公式变形为:

$$
\begin{aligned}
\frac{d I}{d \omega}(u, \omega)= & \frac{1}{\pi^{2}} \frac{q^{2}}{c} \alpha^{2} N \frac{1}{\sqrt{2 \pi \sigma_{x}^{2}}} \frac{1}{\sqrt{2 \pi \sigma_{y}^{2}}} \iint K_{1}^{2}\left(\alpha \sqrt{\left(u_{x}-x^{\prime}\right)^{2}+\left(u_{y}+b-y^{\prime}\right)^{2}}\right) \\
& * \exp -\left[x^{2} / 2 \sigma_{x}^{2}\right] \exp -\left[y^{2} / 2 \sigma_{y}^{2}\right] d x d y
\end{aligned}
$$

水平极化分量分布公式变为:

$$
\begin{aligned}
\frac{d I}{d \omega}(u, \omega) & =\frac{1}{\pi^{2}} \frac{q^{2}}{c} \alpha^{2} N \frac{1}{\sqrt{2 \pi \sigma_{x}^{2}}} \frac{1}{\sqrt{2 \pi \sigma_{y}^{2}}} \iint \frac{\left(u_{x}-x^{\prime}\right)^{2}}{\left(u_{x}-x^{\prime}\right)^{2}+\left(u_{y}+b-y^{\prime}\right)^{2}} \\
& * K_{1}^{2}\left(\alpha \sqrt{\left(u_{x}-x^{\prime}\right)^{2}+\left(u_{y}+b-y^{\prime}\right)^{2}}\right) \exp -\left[x^{2} / 2 \sigma_{x}^{2}\right] \exp -\left[y^{2} / 2 \sigma_{y}^{2}\right] d x d y
\end{aligned}
$$

坚直极化分量分布公式变为:

$$
\frac{d I}{d \omega}(u, \omega)=\frac{1}{\pi^{2}} \frac{q^{2}}{c} \alpha^{2} N \frac{1}{\sqrt{2 \pi \sigma_{x}^{2}}} \frac{1}{\sqrt{2 \pi \sigma_{y}^{2}}} \iint \frac{\left(u_{y}+b-y^{\prime}\right)^{2}}{\left(u_{x}-x^{\prime}\right)^{2}+\left(u_{y}+b-y^{\prime}\right)^{2}}
$$




$$
* K_{1}^{2}\left(\alpha \sqrt{\left(u_{x}-x^{\prime}\right)^{2}+\left(u_{y}+b-y^{\prime}\right)^{2}}\right) \exp -\left[x^{2} / 2 \sigma_{x}^{2}\right] \exp -\left[y^{2} / 2 \sigma_{y}^{2}\right] d x d y
$$

这里, ( $\mathrm{ux}, \mathrm{uy}+\mathrm{b})$ 是在 ODR 屏上的观察点坐标, $\mathrm{b}$ 是作用因子, $\sigma_{x}, \sigma_{y}$ 是电子 束两个方向的束斑大小。

基于公式(5.8), 束流倾斜角为 $45^{\circ}$ 时总的投影强度分布如下所示

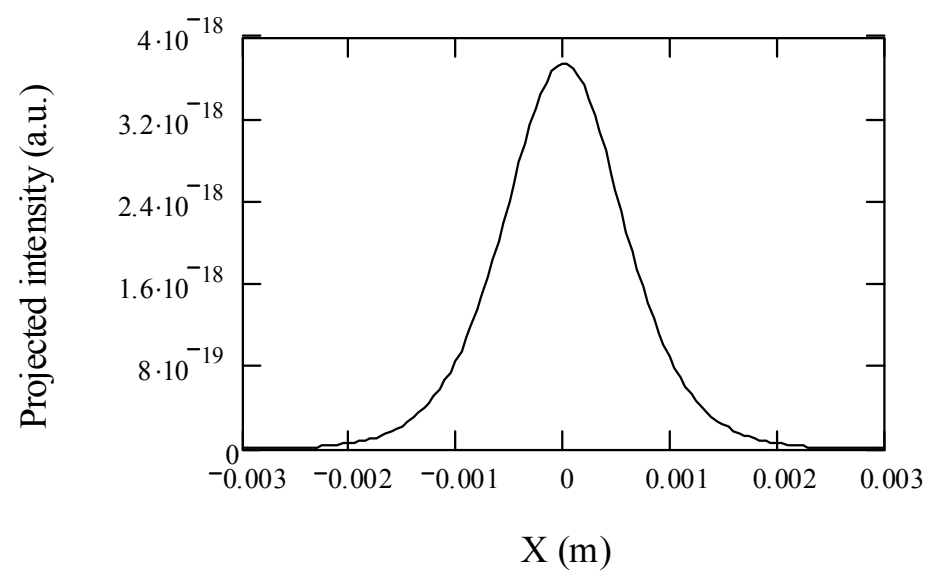

图 5.16 束流倾角 $45^{\circ}$ 时衍射辐射投影分布

可见，尽管束流倾斜，其总的投影分布也是近高斯分布。

不同倾斜角度下总的投影分布为

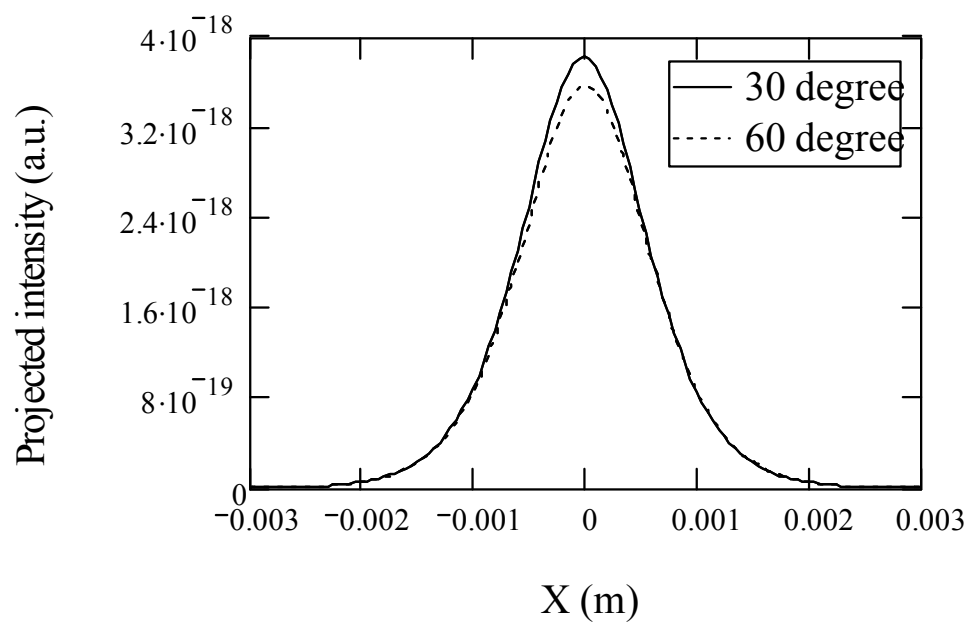

图 5.17 不同倾角对辐射投影分布的影响

随着倾斜角度增大时，强度分布的峰值有些许下降，但始终保持近高斯分布。 基于，倾斜束流产生的辐射水平极化分量如下: 


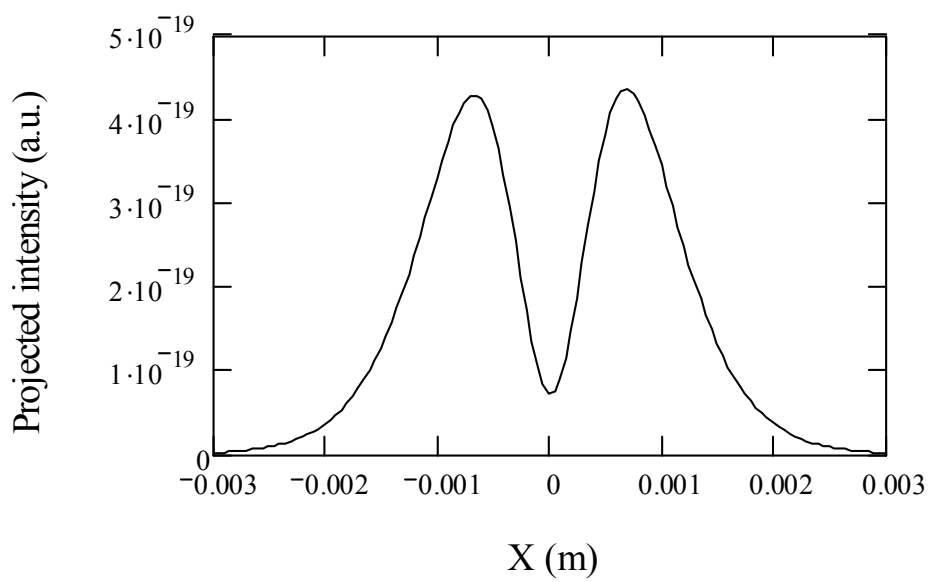

图 5.18 倾角 $45^{\circ}$ 时水平衍射辐射分量分布

和经验预期不一样, 水平极化分量的两个峰强度几乎一致。进一步的计算告诉我 们, 倾斜角度为 $45^{\circ}$ 时, 右侧峰比左侧峰略强 $2 \%$ 。同样, 我们发现倾斜角度从 $30^{\circ}$ 到 $60^{\circ}$ 的变化使得两个峰值都有所下降。

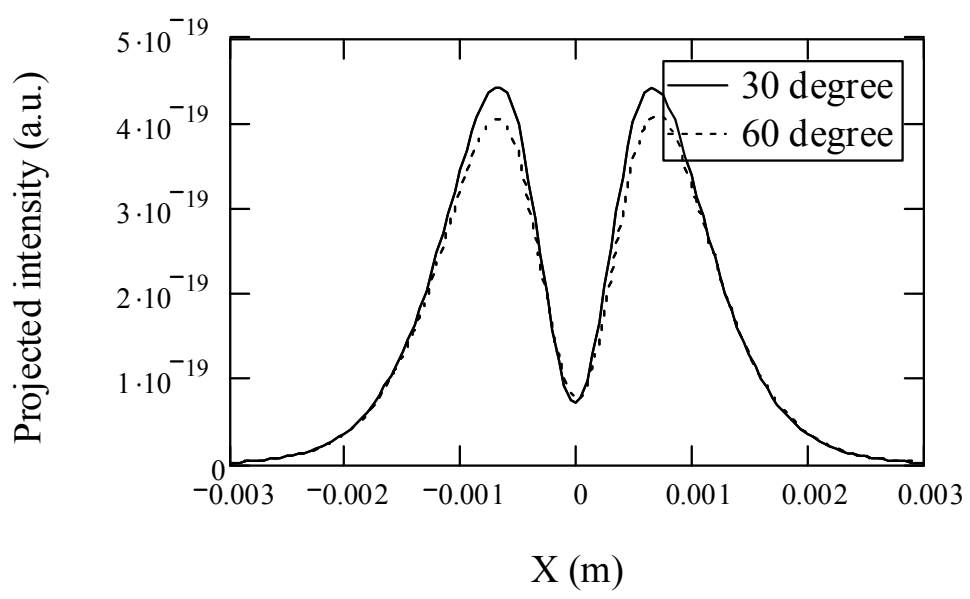

图 5.19 不同倾角对于水平衍射辐射分布的影响

需要指出的是, 以上是对特定波长 $(500 \mathrm{~nm})$ 计算的结果。为了更好地模拟 实际测量, 需要对可见光范围进行积分。我发现积分后给出的结论和现在的一致: 倾斜截面的电子束产生的衍射辐射水平极化分量有着几乎对称的分布。因此, 上 面所提到的解释是行不通的, 我们需要更合理的解释。

自然, 我们会将审查的重点转移到影响辐射分布的外部因素——光学成像系 统。相对于其他的光学元件, 极化波片是产生极化分量的直接元件, 因此它成为 第一个检验的对象[62]。可以理解, 我们检验的将是波片最重要的参数一一传输 线的方向。对于理想的水平极化波片, 其作用可以用一二维矩阵表示 


$$
\operatorname{Pol}_{x}=\left(\begin{array}{ll}
1 & 0 \\
0 & 0
\end{array}\right)
$$

理想波片被假设为没有吸收损耗、且传输线与 $\mathrm{x}$ 轴完全重合。如果实际极化 波片的传输线和 $\mathrm{x}$ 轴成 $\theta$ 角度, 二维矩阵则变为:

$$
\mathrm{Pol}_{x}^{\prime}=\left(\begin{array}{cc}
C_{\theta} & -S_{\theta} \\
S_{\theta} & C_{\theta}
\end{array}\right)\left(\begin{array}{ll}
1 & 0 \\
0 & 0
\end{array}\right)\left(\begin{array}{cc}
C_{\theta} & S_{\theta} \\
-S_{\theta} & C_{\theta}
\end{array}\right)=\frac{1}{2}\left(\begin{array}{cc}
1+C_{2 \theta} & S_{2 \theta} \\
S_{2 \theta} & 1-C_{2 \theta}
\end{array}\right)
$$

在该极化波片的作用下，传输的场强可以表示为

$$
\left(\begin{array}{c}
E_{x}^{\prime} \\
E_{y}^{\prime}
\end{array}\right)=\frac{1}{2}\left(\begin{array}{cc}
1+C_{2 \theta} & S_{2 \theta} \\
S_{2 \theta} & 1-C_{2 \theta}
\end{array}\right)\left(\begin{array}{c}
E_{x} \\
E_{y}
\end{array}\right)
$$

其中 $\left(\begin{array}{l}E_{x} \\ E_{y}\end{array}\right)$ 是电场的两极分量。那么, 通过该极化波片的辐射强度可以表示为:

$$
I_{H P}=\left(E_{x}{ }^{\prime}\right)^{2}+\left(E_{y}^{\prime}\right)^{2}
$$

结合前面讨论的辐射极化分量的分布, 我们可以很容易给出任意方向波片作 用下的辐射分量的表达式。下图给出了波片传输线与 $\mathrm{x}$ 轴夹角分别为 $5^{\circ} 、 10^{\circ}$ 时的投影强度分布

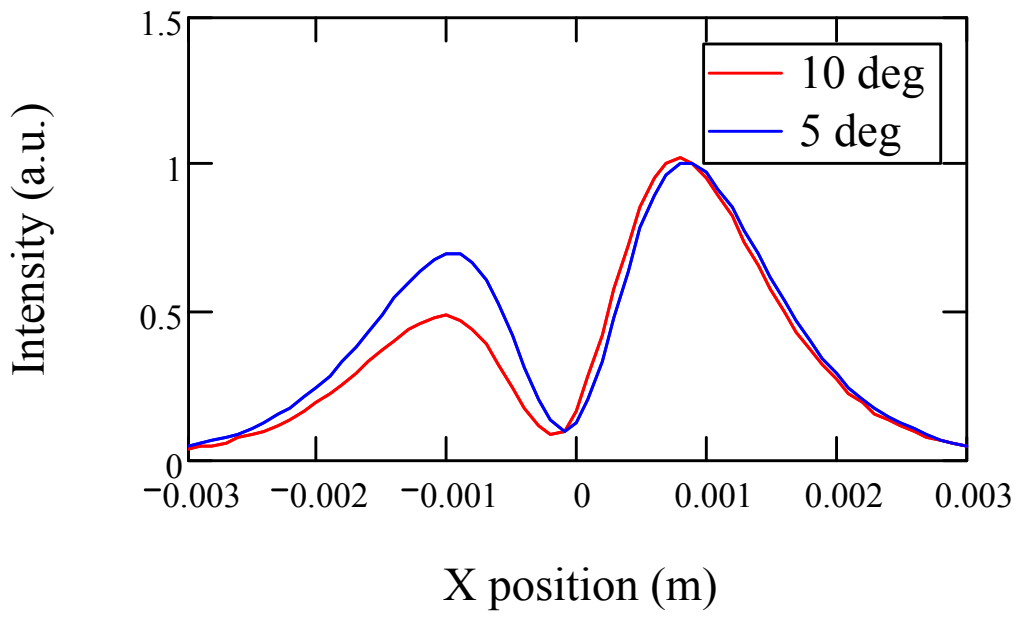

图 5.20 波片倾角对水平衍射辐射分布的影响

可见，水平极化分量分布对于波片传输线取向特别敏感，角度为 $5^{\circ}$ 时峰值 差别高达 $30 \%$ 。如前面介绍的那样, 波片被安装在被远程控制的可翻转架上, 极 有可能波片被安装时出现了些许偏差。下面给出实验测量的对照。 


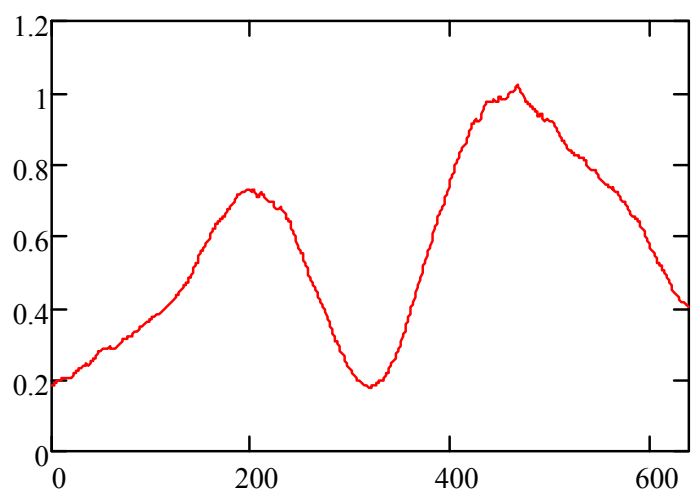

图 5.21 实验测量水平衍射辐射分布

上面是实验测量的水平极化分量的投影分布, 双峰强度相差略小于 $30 \%$, 因 此可以判断水平极化波片传输线偏离 $\mathrm{x}$ 轴略小于 $5^{\circ}$ 角。

除了安装时可能出现的误差外, 波片上所注明的传输线方向或许与实际方向 有出入, 因此一次性的纠正可能无法彻底解决问题。如果为波片使用一个可转动 的安装座, 那么就可以实时调节以彻底消除分布的不对称性。

\subsection{OTR测量中的极化波片}

第二章测量精度的讨论告诉我们: 水平极化波片将会压缩束斑坚直分布; 坚 直极化波片会压缩束斑水平分布。测量结果第一次明确地印证了这个结论, 如图 5.22 及图 5.23 所示。

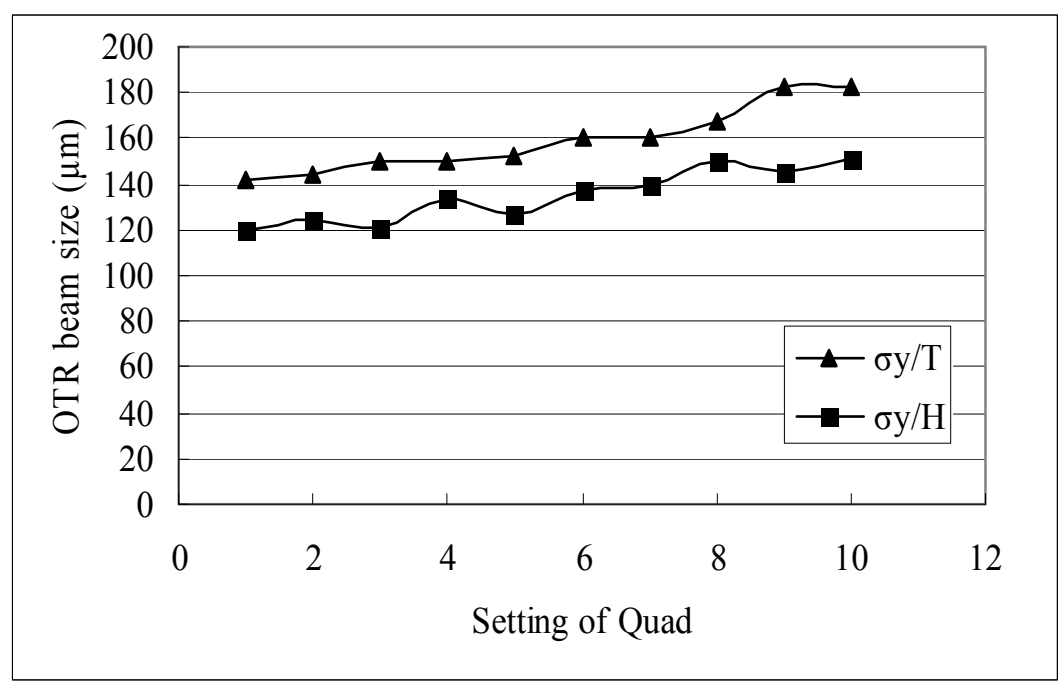

图 5.22 水平极化波片对 OTR 坚直束斑测量的影响

以上是在连续变化四极铁强度的同时进行的 OTR 测量。位于上部的数据点 
是没有极化波片测量下的坚直束斑大小, 位于下部的数据点是在有水平极化波片 时的坚直束斑大小。可见, 水平极化波片对坚直束斑的压缩量达 20 30um。

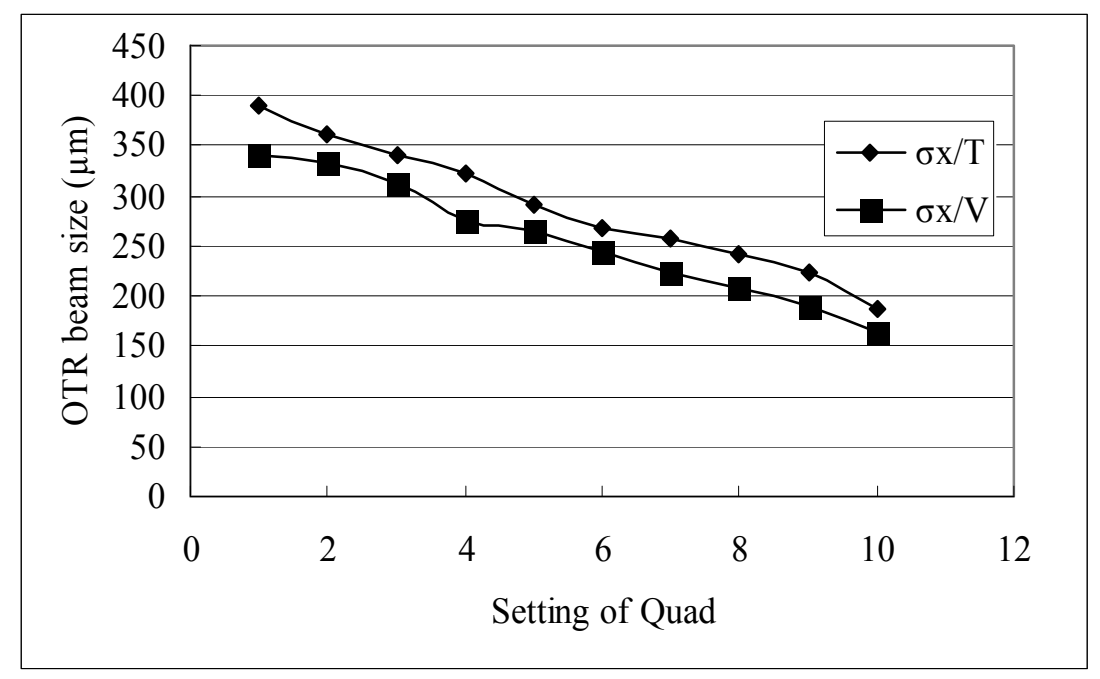

图 5.23 坚直极化波片对 OTR 水平束斑测量的影响

同样，图中位于上部的数据点是没有极化波片测量到的水平束斑大小，位于 下部的是有坚直极化波片测量到的水平束斑大小。坚直极化波片对水平束斑的压 缩量达 15 40um。

\subsection{OTR测量和坚琴测量}

在测量中束斑大小的改变是通过 $\mathrm{Q}$ 铁强度的改变来实现的。我们依据的程序 如下: 连续改变 $\mathrm{Q}$ 铁强度, 取 10 个值, 利用 OTR 测量束斑大小, 同时记录 $\mathrm{Q}$ 铁设置, 完成 OTR 测量后, 重复 $\mathrm{Q}$ 铁参数重复坚琴测量。下面是两种测量结果 的比较

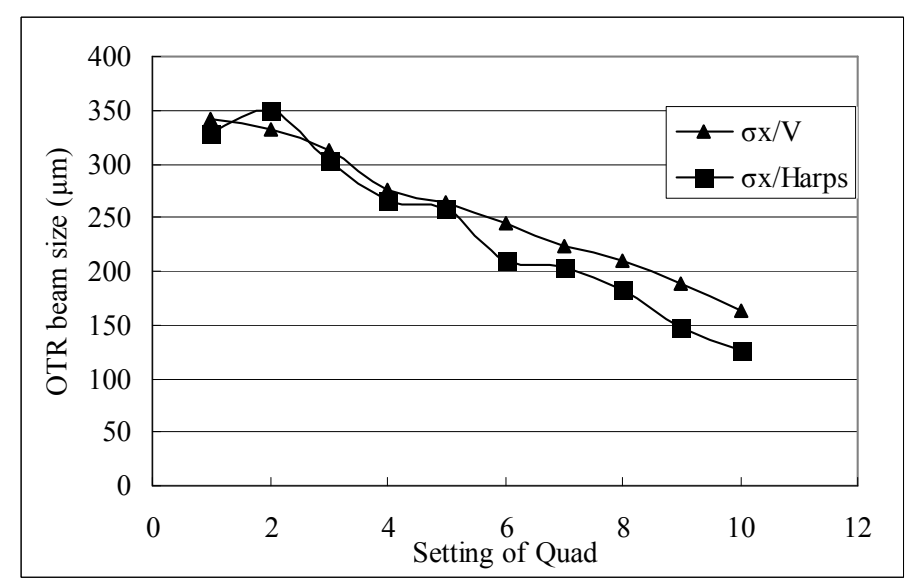

图 5.24 四极铁扫描下 OTR 和坚琴对水平束斑测量结果比较 


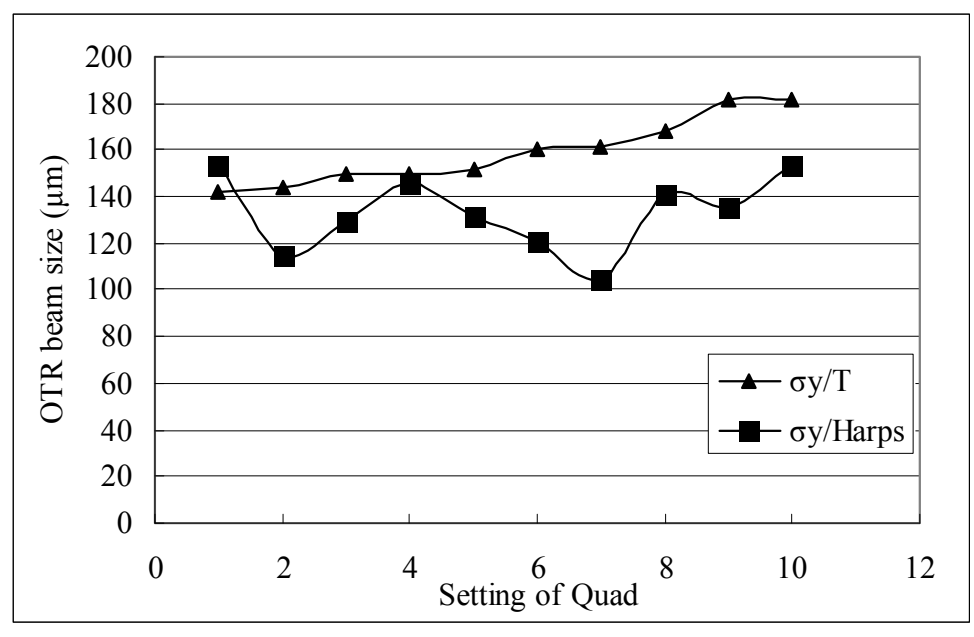

图 5.25 四极铁扫描下 OTR 和坚琴对坚直束斑测量结果比较

尽管 $\mathrm{Q}$ 铁强度的选取没有线性规律，我们也不预期束斑大小的变化是线性 的。但是, 随着 $\mathrm{Q}$ 铁强度的递增（减）变化, 束斑大小的变化在一个方向上应 该是递增而另一个方向上应该递减。不管是水平方向还是坚直方向上束斑的测 量, 坚琴测量数据点的分布都出现了很大的跳跃性。因此, 坚琴测量的结果并不 能很好地反映束斑大小的真实变化。相反, OTR 测量给出了很平滑的束斑变化。 因此, 相比而言, 更有理由相信 OTR 测量能给予更为准确的束斑尺寸。在只有 坚琴和 OTR 测量两种绝对测量手段的情况下, 我们取 OTR 测量作为衍射辐射测 量的参考。

\section{7 ODR测量和OTR测量}

对应于 $\mathrm{Q}$ 铁扫描的 OTR 测量和坚琴测量, 我们也进行了相同 $\mathrm{Q}$ 铁扫描下的 ODR 测量。如上一章所介绍, 我们分别做了只有中性滤波片的测量、加水平极 化波片的测量、加坚直极化波片的测量和窄带滤波片的测量。通过这些测量结果 和 OTR 测量（被当作实际束斑大小）的比较, 我们会总结出将 ODR 测量发展 成束斑测量手段的方法。

图 5.26 中, OTR 测量的水平束斑大小（实际水平束斑大小）被当作 $\mathrm{x}$ 轴, 经过处理得到的 ODR image size 被当作 $\mathrm{y}$ 轴。可以看出, ODR 测量到的束斑大 小和实际尺寸有着近似线性的关系。线性拟合的结果是： $y=1.5607 x+774.08$, 拟合优度 $R^{2}=0.9604$ 。 


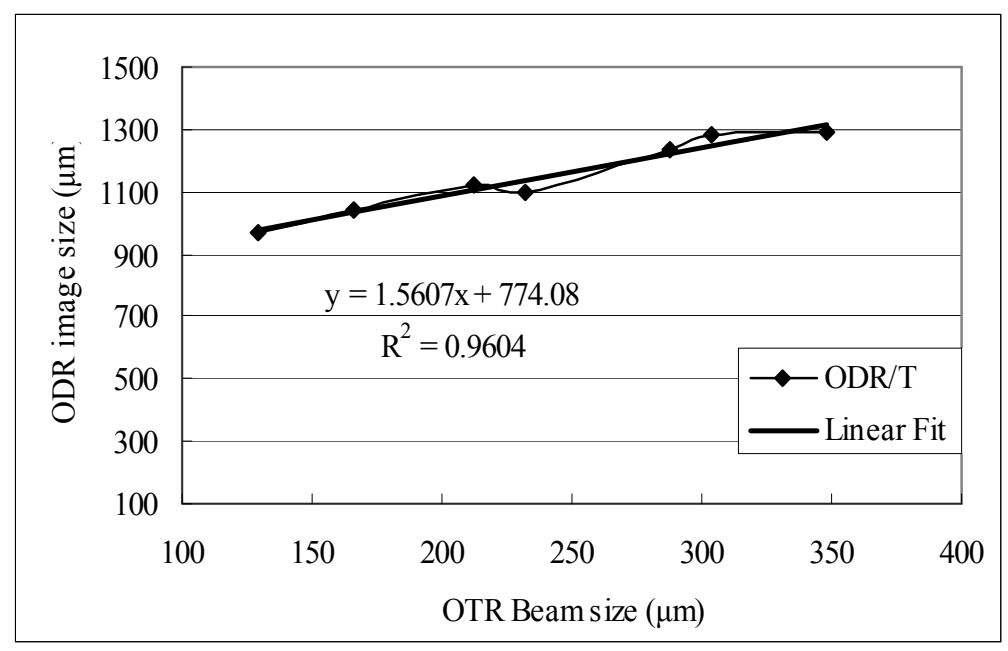

图 5.26 四极铁扫描下 ODR 测量与 OTR 测量的依赖关系

下面是有坚直极化波片的测量结果的比较

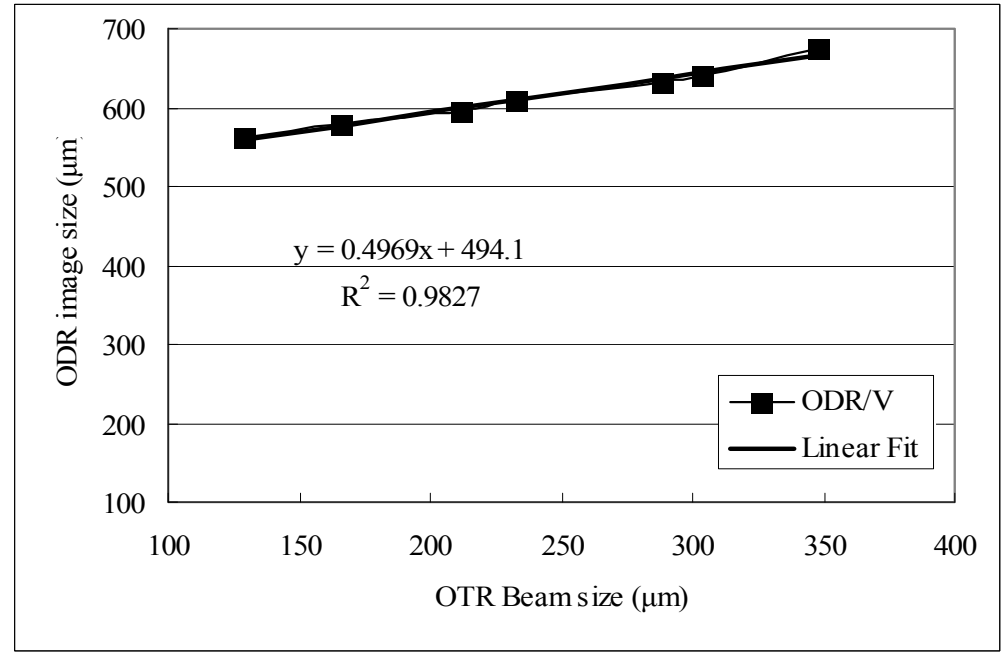

图 5.27 四极铁扫描下 ODR 测量（带坚直极化波片）与 OTR 测量的依赖关系

很明显, 在有坚直极化波片的测量中, ODR image size 和实际束斑大小有着 更好的线性关系。这样的效果得益于我们前面所讨论过的极化波片对于点发散函 数的作用。以上只是其中两次测量的结果, 我们所进行的三次 $\mathrm{Q}$ 铁扫描均给出 了相同的结论。

很自然, ODR 测量水平束斑将会需要如下的程序: 进行相同光学设置下的 Q 铁扫描, 分别进行在调节模式下 OTR 测量和连续模式下的 ODR 测量, 拟合找 到两者间的线性关系, 在机器正常运行下进行 ODR 在线测量, 通过线性关系计 算真实束斑大小。

在 $\mathrm{Q}$ 铁扫描中, 我们也进行了有窄带滤波器的测量。如前面一章所给出的测 
量图像所示, $650 \mathrm{~nm} 、 750 \mathrm{~nm}$ 窄带测量的强度很弱, 而长通和短通滤波片的测量 达到了饱和, 都影响了测量数据的分析。下面给出的是 $550 \mathrm{~nm}$ 窄带测量的结果:

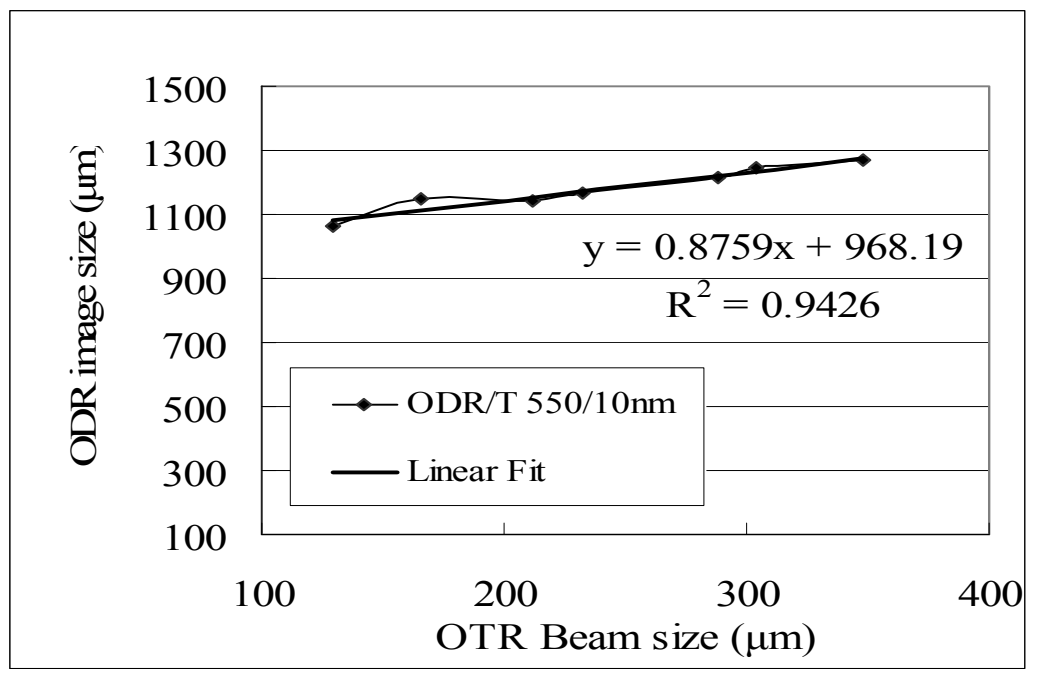

图 5.28 四极铁扫描下 ODR 测量（带 $550 / 10 \mathrm{~nm}$ 波片）与 OTR 测量的依赖关系

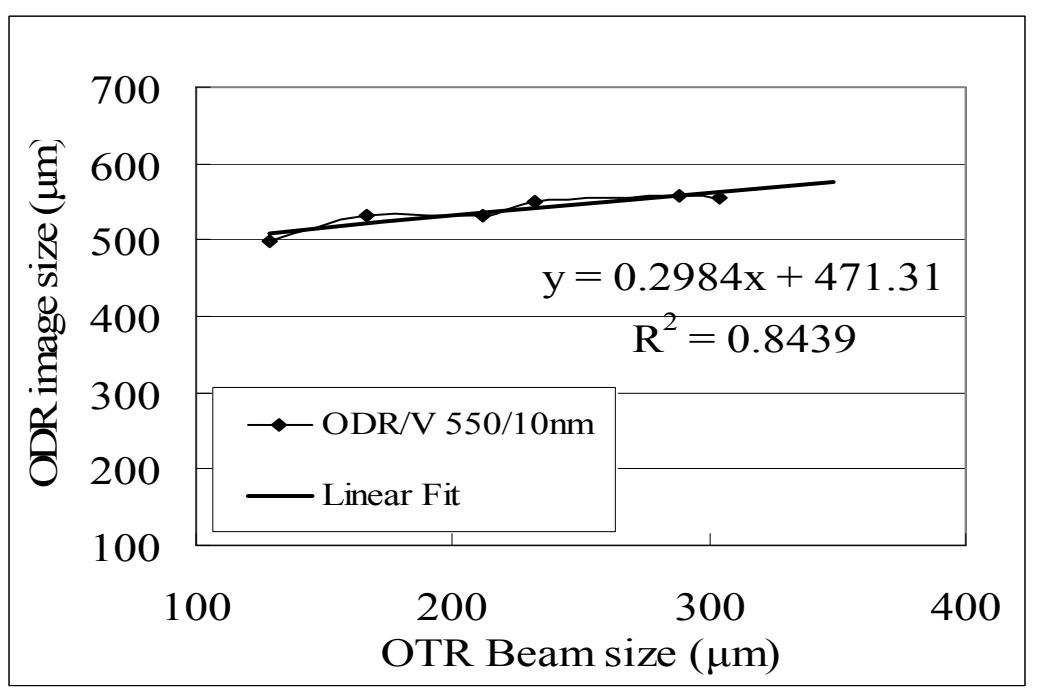

图 5.29 四极铁扫描下 ODR 测量（带 $550 / 10 \mathrm{~nm}$ 波片及极化波片）

与 OTR 测量的依赖关系

图 5.29 中因为一个数据点的缺失导致了拟合优度的恶化，但总体数据的分布同 样展现了很好的线性关系。在高电流强度的机器中, 窄带滤波器的消色差功能可 以被运用到 ODR 测量中。

\section{8 波长和电流依赖关系}

依据 ODR 辐射模型, 通过计算得到了不同波长下的辐射分布 


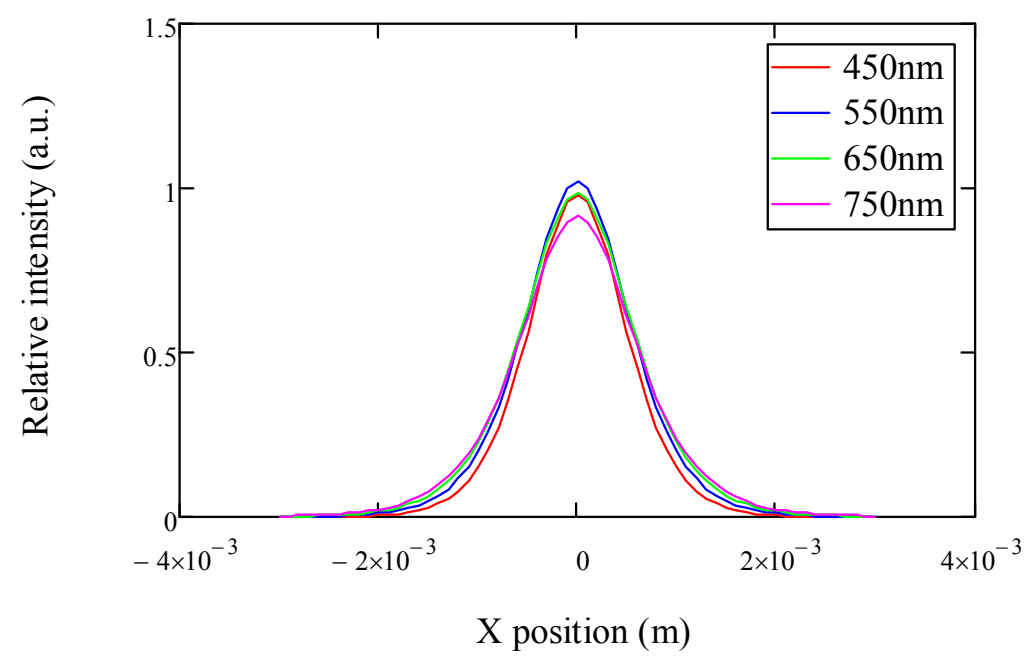

图 5.30 不同波长下辐射强度比较

可以看到, 在 $550 \mathrm{~nm}$ 波长处的辐射相对更强一些。这和我们的测量是一致的。 另外, 考虑到 $\mathrm{CCD}$ 相机的频率响应, 响应峰值位于 $500 \mathrm{~nm}$ 并随波长减小或增加 逐渐下滑, 因此出现了辐射在长波处的衰弱。但是, 理论上来讲, 辐射强弱并不 会影响分布的宽窄。下面是模拟的分布结果

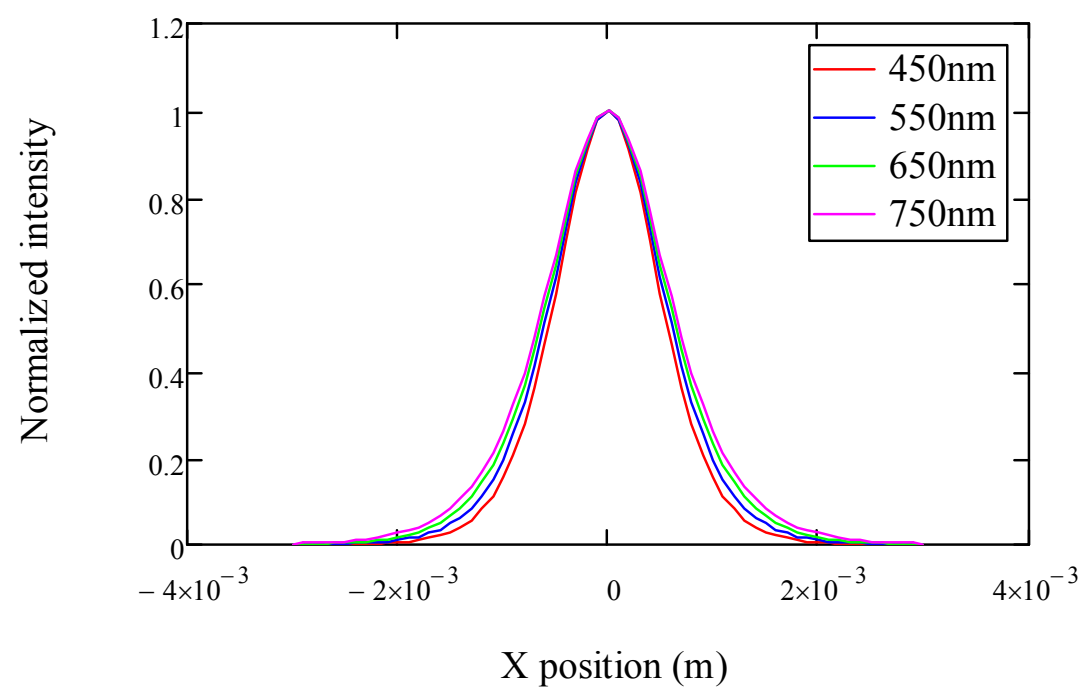

图 5.31 不同波长下的辐射相对分布

可以看出, 辐射分布会随着波长的增加变宽, 这一点不应该随着强度的变化而变。 图 5. 31 是 ODR 测量 image size 随波长的变化。可以看到, 无论是总辐射测量 还是有坚直波片的测量, 其随波长的变化在长波处都与理论计算出现了不一致。 我认为其原因在于：长波长处观察到的辐射因 $\mathrm{CCD}$ 相机的响应低而很弱, 导致 其容易受到 CCD 本底（辐射本底已被减除）的影响, 辐射分布的基底被本底淹 
没, 导致分布变窄。

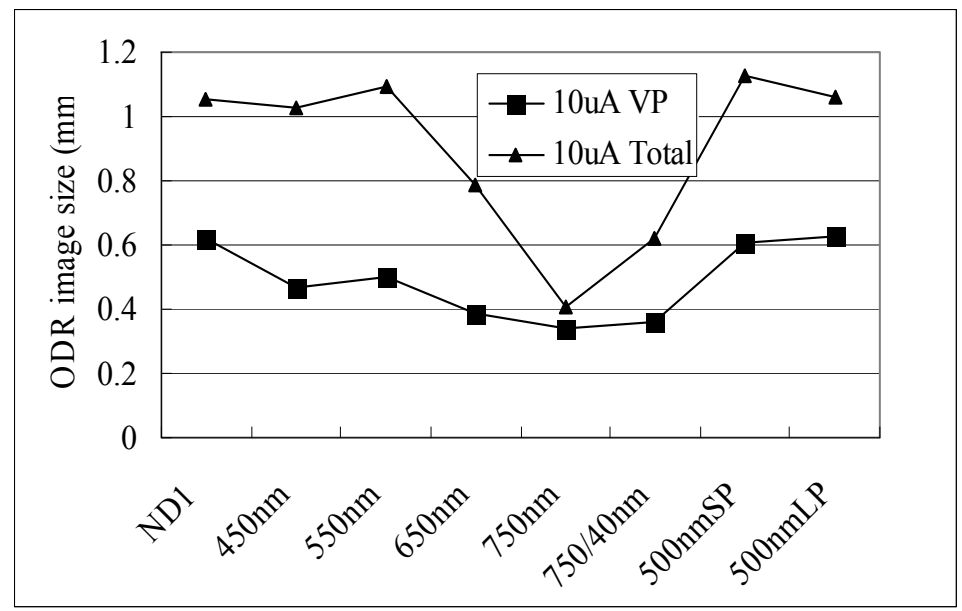

图 5.32 不同波长处的 ODR 束斑测量结果, 三角表示

为了研究 ODR 辐射测量的适用范围, 我们也进行了不同束流强度条件下的 测量

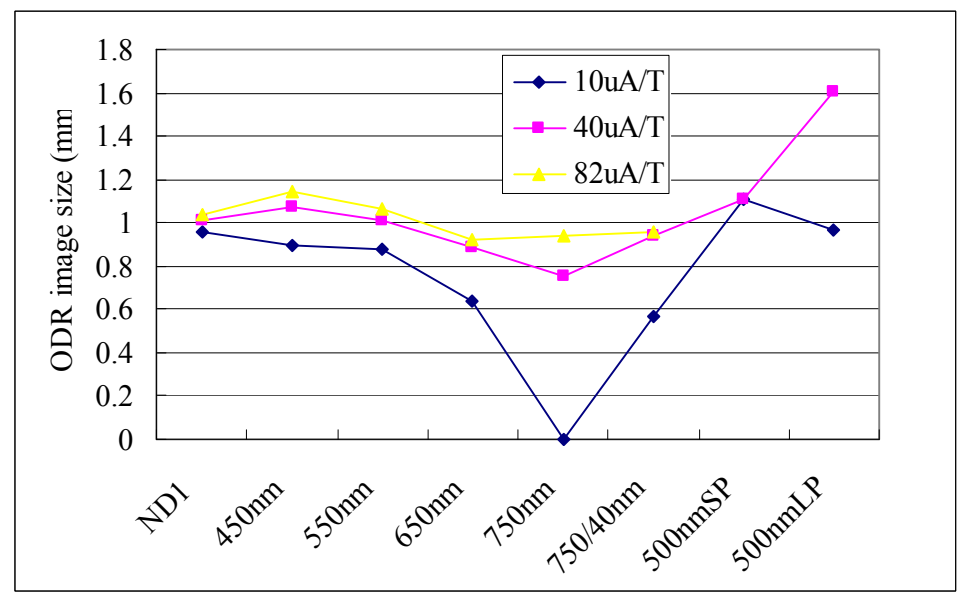

图 5.33 ODR 测量结果对电流强度依赖关系(无波片)

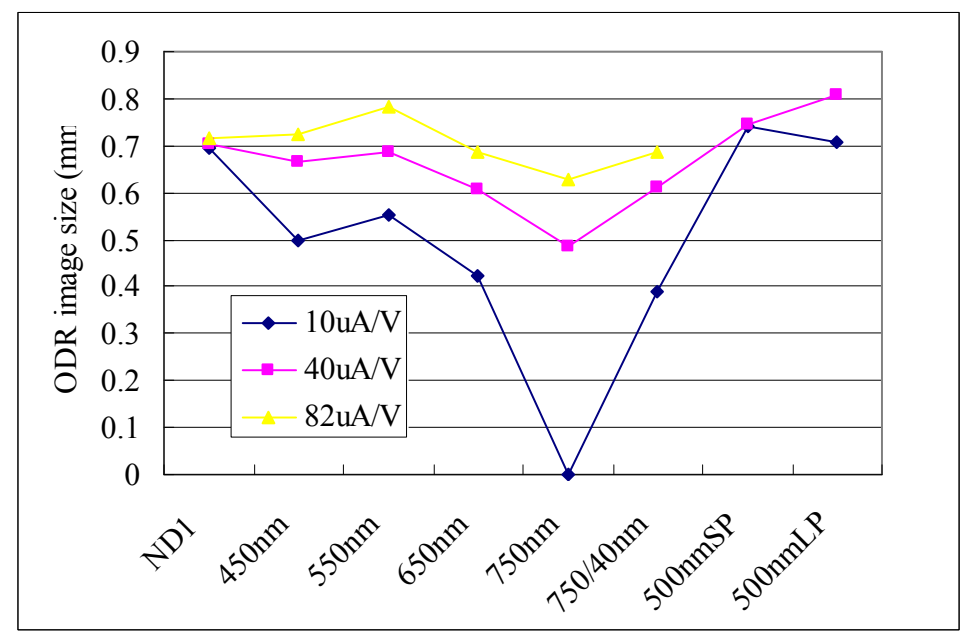

图 5. 34 ODR 测量结果对电流强度依赖关系（有坚直波片） 
图中为零的两点为数据缺失。在我们的测量中束流强度的下限是 $5.4 \mathrm{uA}$, 上 限是 $82 \mathrm{uA}$ 。测量中束流损失监视器（BLMs）没有观察到任何的信号。限于驱动 激光的功率, Hall A 无法运行高于 $82 \mathrm{uA}$ 的连续束流。

\section{9 本章小结}

本章给出了 ODR 辐射测量的全面分析, 包括辐射干扰的来源及减小其影响 的提案, 水平极化分量分布的非对称性的解释和校正办法; 然后通过 OTR 和 Harps 测量的比较, 确定以 OTR 测量的束斑作为实际束斑大小, 以此作为衍射 辐射的定标工具; 得到了 ODR image size 和束斑大小的线性依赖关系, 验证了 ODR 测量是一个可靠的束斑大小测量的办法; 还分析了 ODR 测量对于波长和电 流的依赖关系, 解释了和预期不一致的测量结果。 


\section{第六章 康普顿激光系统简介}

加速器技术和激光技术都是在二十世纪初期开始发展的。经过近一个世纪的 进步, 这两项技术都分别在基础研究、工业生产和日常生活方面起到了举足轻重 的作用。同时, 这两项技术又逐步产生了千丝万缕的联系, 致使现代加速器技术 的发展愈来愈依赖于激光技术。

为了得到高品质的电子束, 激光驱动光阴极被用于电子束流的产生[86-88]。 理论研究发现, 均匀粗球分布 [89]的电子束或驼峰状[90]的电子束在被加速的过 程当中能保持比较低的发射度。因此出现了一些激光整形[91-93]方面的研究工 作, 通过对激光整形达到对电子束整形而实现高品质加速器。作者在激光整形方 面也进行了一些研究, 详细内容可以参考[94]。为了对电子束进行高精度的测量, 出现了一些利用激光技术的诊断方法, 如基于反康普顿散射的激光线扫描和激光 干涉仪, 还有利用晶体普克尔斯效应的光电采样 $[95,96]$ 对电子束纵向分布的测 量。同样基于电子束与激光束的相互作用, 我们可以得到短脉冲的 $\mathrm{x}$ 射线 $[97,98]$ 。 更能说明加速器技术和激光技术的深刻联系的是现今热门的两项研究课题, 一是 加速器产生的激光一一自由电子激光[99-102], 另外是利用激光产生等离子体加 速粒子的加速器 $[103,104]$ 。

随着加速器技术在高能物理及核物理领域的广泛应用, 我们需要越来越多的 高精度束流测量技术为加速器的控制及完善提供必要的依据, 为物理实验提供必 要参数, 成为加速器技术以及物理实验中所必不可少的一部分。而高品质激光系 统则是一些束流诊断系统, 如康普顿电子极化测量仪以及电子束斑测量装置（激 光线扫描、激光干涉仪) 中的最关键元件。高功率、高品质、超稳定和可靠的激 光系统是完成这类高精度测量的前提条件。在 Jefferson Lab, 高精度的电子极化 度的测量是 Hall C 下一个重要实验 Q-Weak 成功的必要条件, 因此我们需要搭建 一套用于康普顿电子极化仪的激光系统。

本章首先介绍康普顿电子极化仪中电子激光相互作用过程一一反康普顿散 射的定义和基本属性; 然后在第二、第三节介绍激光在加速器上的应用对于激光 系统的一般要求, 以及 Hall C 康普顿电子极化仪对激光系统的具体要求; 最后, 介绍增益开关二极管技术及光纤激光技术的发展, 为下一章激光系统的具体介绍 
作铺垫, 以及解释舍弃单激光器选取 MOPA 激光系统配置的原因。

\section{1 反康普顿散射}

一般来说, 大部分涉及到电子束和激光束相互作用的测量过程都是基于反康 普顿作用过程, 出于习惯人们通常用康普顿来通指康普顿和反康普顿效应。严格 而言, 康普顿效应[105]指的是高能光子（x-ray， $\mathrm{\gamma}$-ray）与物质相互作用产生低 能光子的现象, 而反康普顿效应[106]指的是低能光子在与其他物质和粒子相互 作用获得能量产生高能光子的过程。

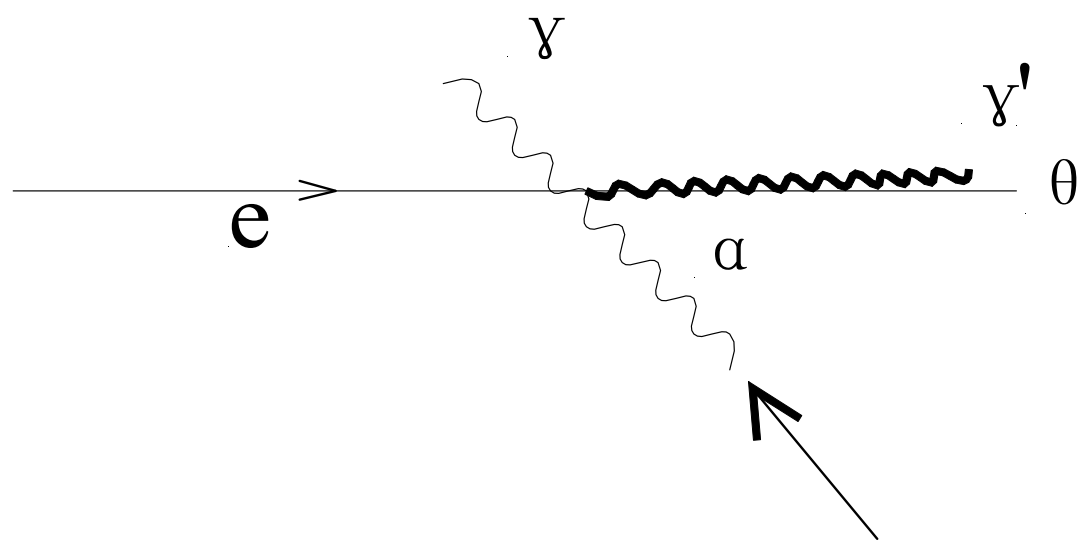

图 6.1 反康普顿散射示意图

如 图 6. 1 是反康普顿散射的示意图, 激光束与电子束以夹角 a 产生碰撞, 被散射光子与电子径迹呈角度 $\theta$ 出射。为了尽量贴近比例, 图中并未体现被散射 电子的散射角。设电子束能量和动量分别为 $\mathrm{E}$ 和 $\mathrm{p}$, 入射光子能量为 $\mathrm{k}$, 散射光子 能量为 $k^{\prime}$, 散射光子能量多普勒上移与出射角度的关系 [107] 是

$$
k^{\prime}=k \frac{E+p \cos \alpha}{E+k-p \cos \theta+k \cos (\alpha-\theta)}
$$

\section{2 激光参数要求}

本节我们将会分析加速器测量应用对于激光系统的一般要求。首先, 激光重 复频率需要能和主加速器频率很好地同步, 因此, 也要求激光重复频率能在主频 率附近灵活可调, 并且时间抖动很小; 其次, 对于激光束的光学品质要求很高, 目的是激光束能在聚焦透镜的作用下达到衍射极限光斑, 提高激光强度从而提高 
散射光子的亮度; 然后, 激光的位置稳定性, 必须小于需要测量的精度; 最后, 激光波长的选择取决于多种因素: 聚焦束斑大小正比于波长, 瑞利长度反比于波 长，康普顿散射截面正比于波长。

表格 6.1 加速器应用对激光系统的要求

\begin{tabular}{ccc}
\hline Parameters & Guidelines & Values \\
\hline Rep. Rate & Accelerator Rep. Rate & MHz to GHz \\
Pulse width & Bync. to reference RF $<$ 1ps rms & \\
Beam quality & TEM00 mode & ps to tens of ps \\
Wavelength & Laser beam size or & $\mathrm{M}^{\wedge} 2 \sim 1$, Gaussian beam \\
& Compton X-section & 500nm to near IR \\
Power & QE of cathode or Compton yields & Ws to tens of W \\
\hline
\end{tabular}

\section{3 Hall C康普顿电子极化仪及激光要求}

电子极化度的测量是通过对反康普顿散射光子谱非对称性的测量来实现的, 因此, 电子束线的设计需要考虑方便于散射光子的探测, 下面是 Jlab Hall C 反康 普顿电子极化仪的总体设计示意图,

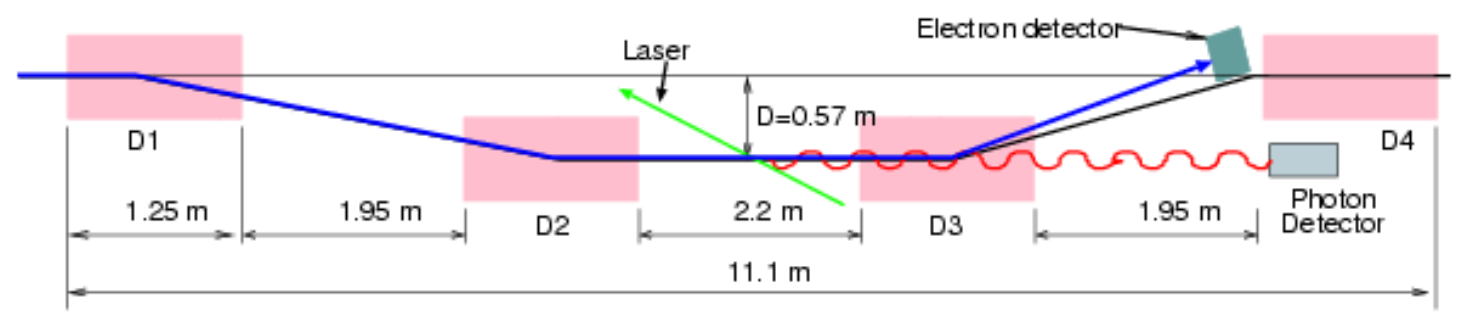

图 6.2 Hall C 康普顿电子极化仪示意图

电子束和激光束的碰撞点位于 Chicane 磁压缩装置的中心, 散射光子运动方向基 本与原电子径迹重合, 进入光子探测器, 第三、四块二极铁间的电子探测器进行 对散射电子的测量并同时起到消除束晕的作用。

选择反康普顿散射作为电子极化测量的最主要原因是该方法的近似非拦截 性, 然而测量必须在电子束状态出现大的漂移前完成, 所以测量需要的时间也是 一个重要考虑的方面。所需时间主要取决于下面三个因素：首先就是散射截面， 前面提到散射截面正比于波长, 所以从此点出发长波长有其优势, 见 图 6. 3; 
然后是散射光子谱的非对称性, 短波长高能激光能提高测量的精度, 见 图 6.4; 最后, 激光和电子束在作用点的亮度, 主要取决于电子束束斑和激光束斑大小以 及相互作用的夹角。综合考虑以上因素, Hall C康普顿电子极化仪对激光参数的 要求列于 表格 6.2 。

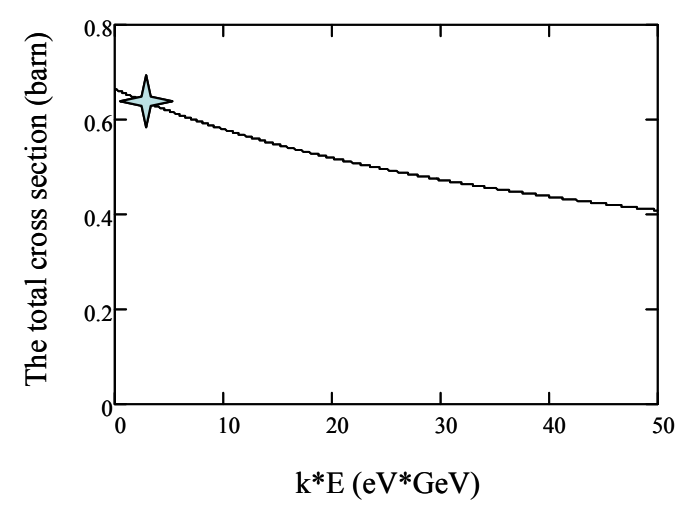

图 6.3 反康普顿散射截面对电子光子能量依赖关系

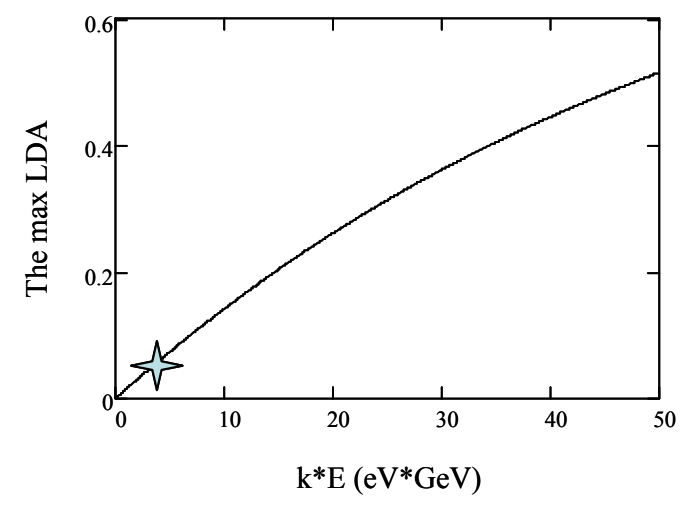

图 6.4 纵向非对称性与电子光子能量的关系

表格 6.2 康普顿电子极化仪对激光的要求

\begin{tabular}{cc}
\hline Parameters & Values \\
\hline Rep. Rate & 499MHz or sub harmonics \\
Pulse width & $10 \mathrm{ps}$ to 30ps rms \\
Beam quality & $\mathrm{M}^{\wedge} 2 \sim 1$, Gaussian beam \\
Wavelength & $532 \mathrm{~nm}$ \\
Power & $>10 \mathrm{~W}$ Ave. \\
\hline
\end{tabular}




\section{4 新激光技术的发展}

随着第一台激光器在上世纪 60 年代的问世, 激光技术出现了翻天覆地的变 化。迄今为止, 激光技术的应用已经深入到工业生产及科学研究的诸多方面。与 其先后发展起来的加速器技术, 也与激光技术的发展形成了强烈的依赖关系, 如 光阴极电子束团的产生、电子参数诊断以及利用加速器技术产生高品质的激光和 利用激光对带电粒子进行加速。

激光新技术依然在不断涌现, 如来自于电信行业的光纤技术也成为激光技术 发展的新动力。光纤激光在很多应用领域都有潜力替代其他的激光技术, 这得益 于其所具有的很多优势: 增益介质具有细长结构, 能提供很高的效率产生很高品 质的激光束, 能产生高功率输出, 没有散热处理和准直的麻烦等等。其在 1um 处的高品质激光输出完全可以取代 Nd: YAG 激光器的地位。高功率加高品质的 光束也使光纤激光成为 $\mathrm{CO}_{2}$ 激光器的强有力的替代。同时, 光纤激光器的发展 是和洜浦二极管激光的技术进步联系在一起的。二极管激光非常小巧, 可以实现 光纤耦合输出, 而且可以工作在连续和脉冲状态。二极管激光的寿命从 10000 小时提升到 20000-30000 小时大大地改善了它的可靠性, 而且, 它的工作效率也 得到了长足的发展。本论文中的激光方面工作同时使用了二极管激光和光纤激光 技术, 所建立的系统紧凑稳定且光品质高, 同时也对加速器领域激光技术的更新 起到了推动的作用。

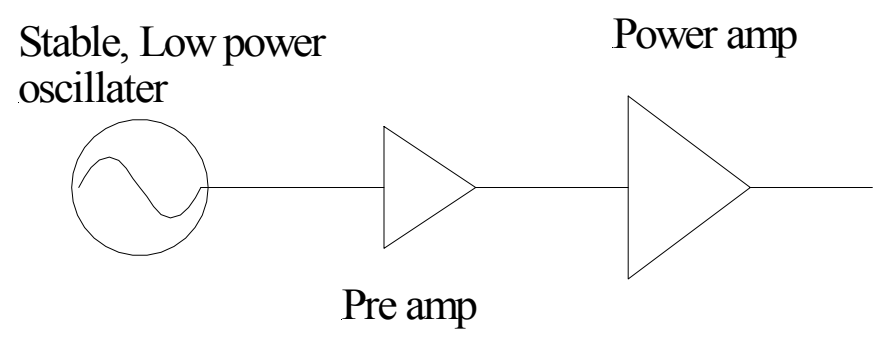

图 6. 5 MOPA 激光系统示意图

\section{Pump}

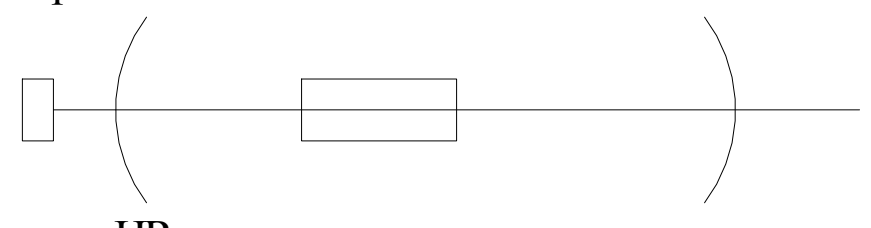

$\mathrm{HR}$

$\mathrm{OC}$

图 6.6 单一激光器系统示意图 
激光系统的性质及工作范围取决于激光系统设置, 一般来讲系统设置分为两 种类型: MOPA (Master Oscillator Power Amplifier) 和单激光振荡器 (Single Oscillator)。图 6.5 及图 6.6 分别是这两种激光系统的示意图。MOPA系统具有 以下性质: 容易对激光参数 (波长、激光束团长度、重复率) 进行调节, 有能量 升级的空间, 单个元件的故障不会导致整个系统的㿈疾, 稳定坚固受机械振动影 响小, 缺点是能量转换效率低。相对而言单激光振荡器的特点是: 难于控制, 单 个元件故障会使系统崩溃, 受外界影响大, 优点是高能量转换效率。鉴于二者的 性质差异和康普顿电子极化仪以及其他诊断系统对于激光功率的高要求, MOPA 激光系统设置成为必然的选择。

\section{5 本章小结}

本章是论文第二部分工作的一个绪论, 为了突出第一部分工作的重要性, 这 一部分的介绍相对简单。主要是介绍了下一章将要详细介绍的激光系统应用的大 环境一一康普顿电子极化仪, 以及其对激光系统的具体要求。 


\section{第七章 $5 \mathrm{~W}$ 激光系统}

根据 MOPA 激光系统和单激光器性能及优缺点的比较, 我们认为 MOPA 激 光系统在参数上能更好地满足加速器激光应用的需要, 而且价格成本更低; 另外, 从科学研究的角度来讲, MOPA 激光系统能提供一些有兴趣的研究课题。因此, 我们使用了该激光系统设置, 其主要组成部分是: 增益开关激光源

(Gain-switching seed), 5W 光纤激光放大器 (Fiber Amplifier), 三硼酸锂倍频 器 (LBO Doubler)。

本章依据激光生成的先后顺序, 对所搭建的 $5 \mathrm{~W}$ 激光系统的激光源、放大器 和倍频器分别进行介绍。第一步部分对各种脉冲激光技术进行总结和比较, 确定 了使用增益开关二极管激光作为系统的主振荡器, 给出了我所组装的增益开关二 极管的硬件介绍, 以及在该激光源上所实现的两项新技术一一脉冲选择和脉冲生 成; 第二部分是关于光纤放大器的介绍和在放大器出口进行的激光传输测量; 第 三部分在不同的倍频晶体比较的基础上, 确定了使用可以实现非临界匹配的 LBO 晶体, 给出了不同情况下的倍频实验结果。然后, 利用数值模拟对实验结 果进行了验证, 而且对高功率下的倍频结果作了预计, 从而确定了使用 Nd: YVO4 晶体放大器将功率放大至 $50 \mathrm{~W}$, 在预计的倍频效率下得到 $15 \mathrm{~W}$ 左右的绿光, 达 到康普顿电子极化仪对激光功率的要求。

\section{1 激光源技术}

激光系统的 MOPA 设置类似于加速器系统的注入器加主加速器的设置。作为 加速器领域的研究人员, 我们深刻体会整台加速器的性能极大程度被注入器束流 的性能所决定和限制。类似地, 激光源也是激光系统中的最关键组成部分 [108]。 因此, 我们在激光源的选择上非常审慎。考虑到激光重复率要和电子重复率或分 频相匹配的要求, 我们考虑了所有能产生脉冲激光的技术, 包括锁模激光器 ( Mode-locking Laser)、Q 开关激光器 ( Q-switching Laser)、倾腔激光器 （Cavity-dumping Laser）和增益开关激光器（Gain-switching Laser）等多种技术 [109]。下面将分别讨论对这些脉冲激光技术取舍的原因。 


\section{1.1 锁模激光技术}

尽管在有些应用中需要纵向单模激光, 但纵向多模激光有产生高输出功率的 潜力。如果在保持高能量的条件下实现超短脉冲的输出形式, 我们就可以同时得 到短的激光脉冲和高的峰值功率。激光锁模技术就可以帮我们实现这个目的。

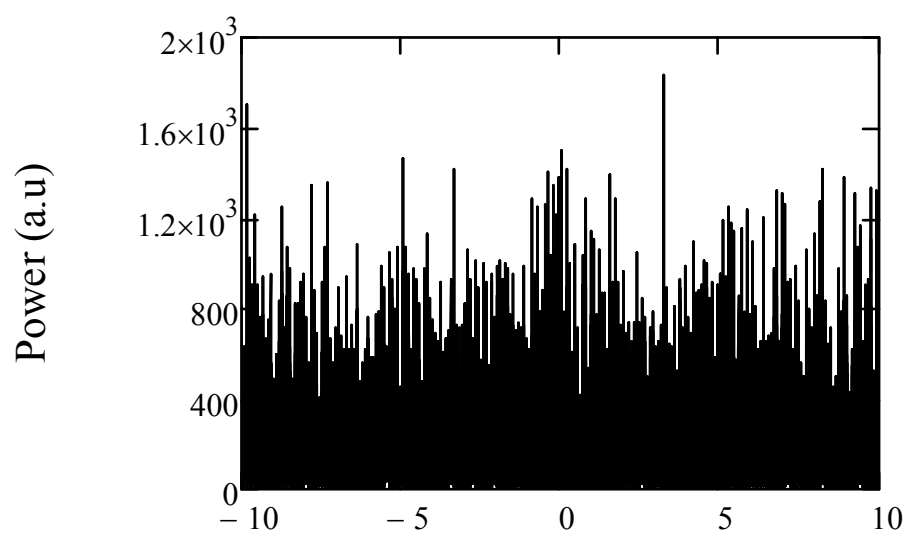

Time (s)

图 7.1 无相位关联电场叠加的时间特性, $\mathrm{N}=201, \mathrm{E} 0=1, \Delta v=1 / 8$

考虑大量纵向模式在激光器中振荡的情形, 假设所有的模式振荡幅度（E0）相 同。设 $\Delta v$ 是相邻纵模的频率差。如果这些纵模之间没有任何相位的关联, 那么 所有模式的叠加就如 图 7.1 所示。其杂乱分布下隐藏着一些规律: 波形是以 $\tau$ $\mathrm{p}=1 / \Delta v=8$ 为周期的函数, 总的输出功率是 $\mathrm{N} * \mathrm{E} 0^{2}$ 。

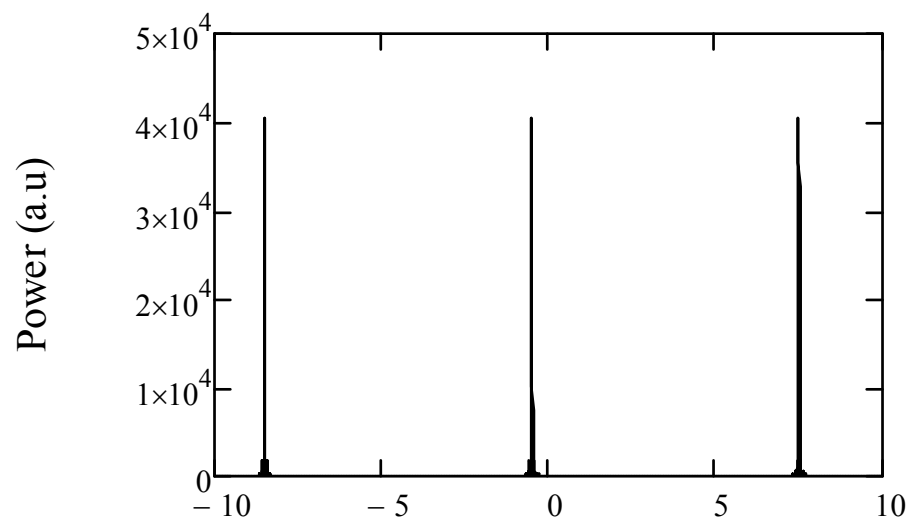

Time $(s)$

图 7.2 纵向电场锁模的输出功率随时间变化

现在我们看看当这些纵模相位存在关联, 即被锁模时的输出功率情形。假设相邻 纵模的相位差恒定，如 $\varphi_{k}-\varphi_{k-1}=\pi / 8$ 。那么总的电场可以表示为 


$$
E(t)=\sum_{k=-n}^{n} E_{0} \exp \left\{j\left[\left(\omega_{0}+k * \Delta \omega\right) t+k \varphi\right]\right\}
$$

该电场强度的平方随时间变化的特性如 图 7.2。以上两种情形的对比还存在下 面的关系: 锁模输出的峰值功率是非锁模条件下的 $2 n+1$ 倍, 模式越多峰值能量 越强; 脉冲之间间隔 $\tau \mathrm{p}=2 \pi / \Delta \omega=1 / \Delta v=8$, 大的脉冲之间存在小的脉冲振动; 脉 冲的半高全宽为 $\tau p=2 \pi /(2 n+1) \Delta \omega=1 / \Delta v_{L}, \Delta v_{L}=(2 n+1) \Delta \omega / 2 \pi$, 模式越多脉冲 越窄。这些就是实现锁模激光的基本思想。

锁模方法可以分为主动和被动锁模。主动锁模又包括幅度调制锁模、频率调 制锁模和同步泵浦锁模, 其中利用普克尔斯盒（Pockels cell）或声光调制器 (acousto-optic modulator) 的幅度调制技术是最常用的。快速可饱和吸收器（fast saturable absorber modulator)、慢速可饱和吸收器 (slow saturable absorber modulator）及科尔透镜（Kerr lens）可以用于实现被动锁模[110]。

\subsubsection{Q开关激光器}

$\mathrm{Q}$ 开关激光器 ( Q-Switching Laser) 中的 Q 指的是激光腔的品质 (Quality)。 激光的产生需要激光腔满足一个基本条件: 激光腔内的增益大于腔的损耗。如果 我们在保持㫤浦激光不变的条件下有意破坏产生激光的条件, 这时激光介质中的 粒子数反转会非常高。在此期间, 激光腔的损耗很高, 腔品质 (Q) 很低。然后 我们恢复正常的发光条件, 即快速调节腔品质 $(\mathrm{Q})$ 到很高值, 那么积蓄在介质 材料中的能量就会在极短的时间内释放出来, 激光输出达到很高的峰值并使得粒 子数反转降低到阈值以下。通过这种方法就可以产生高能的激光脉冲, 该方法的 实现主要在于如何快速调节激光腔的品质 $(\mathrm{Q})$ 。

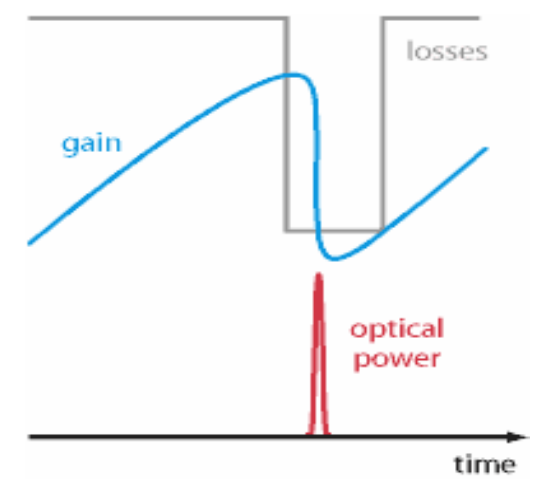

图 7.3 Q 开关技术的原理示意图 
实现 $\mathrm{Q}$ 开关脉冲激光的办法主要有:

电 $\mathrm{Q}$ 开关: 在激光腔中加入 $K D^{*} \mathrm{P}$ 或铌酸锂（Lithium niobate）晶体，并在 晶体两侧施加直流电压利用普克尔斯效应使其折射率发生改变, 以此改变通过晶 体的光的相位, 从而改变腔的品质。

转动棱镜: 激光腔中的棱镜的作用类似于反射镜, 转动棱镜使激光偏离谐振 位置而降低腔的品质 ( $\mathrm{Q})$, 当能量积累到一定程度将棱镜转回而产生激光脉冲。 这种办法简单且适用于任何波长但受制于棱镜转动的速度。

声光 $\mathrm{Q}$ 开关: 在激光腔中放入透明的光学材料, 通过压电传感器在晶体内产 生超声波, 进而在晶体内形成周期折射率分布, 该分布导致部分光被偏离光轴从 而降低激光腔品质, 关掉传感器后, 激光腔恢复正常品质而产生激光脉冲。

可饱和吸收 $\mathrm{Q}$ 开关: 可饱和吸收材料被置入激光光路中, 在一段时间内其吸 收激光然后达到饱和, 在饱和前激光腔的品质非常低, 能量在腔内积累, 饱和后 吸收材料对激光波长变得透明, 激光腔品质恢复形成激光脉冲。

这些 $\mathrm{Q}$ 开关技术都没法产生超短激光脉冲, 一般脉冲长度大于 ns 量级[111]。

\section{1 .3 倾腔激光器}

倾腔激光技术可以和 $\mathrm{Q}$ 开关、锁模激光技术相互结合 $[112,113]$ 。该技术总 的思想是，保持激光腔工作在很低的损耗状态下一段时间，与此同时能量在腔内 积累, 然后用一个光学开关来实现激光脉冲的输出。

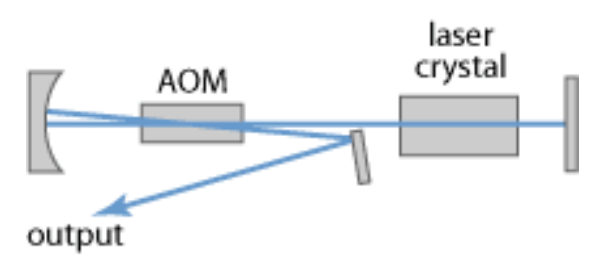

图 7.4 倾腔激光技术示意图

声光调制器和普克尔斯盒就是实现倾腔技术常用的光学开关。脉冲的产生过 程是这样的: 首先, 调制器的工作状态使得腔内部分能量被反射镜输出, 这时激 光处于阈值以下而无法发光，泵浦光的能量大部分被储存在激光介质里；然后， 改变调制器的状态, 腔内出现光振荡, 这样腔内功率就会迅速上升; 最后, 恢复 调制器的工作状态, 使得腔内能量被调制器所偏转并由反射镜输出, 此时的输出 
就是高功率的脉冲。

\section{1.4 增益开关激光技术}

当激光的泵浦光源突然被打开后, 激光的输出功率会经历一定时间的张弛振 荡（relaxation oscillations）才能达到稳定的输出, 其张弛振荡期间的表现为一系 列振荡脉冲输出[114], 如图 7.5 所示。第一个振荡脉冲时间宽度是共振腔往返 时间的有限倍数, 因此其长度取决于腔的长度, 对于固体激光器一般为几十个ns, 后续的脉冲会越来越长。

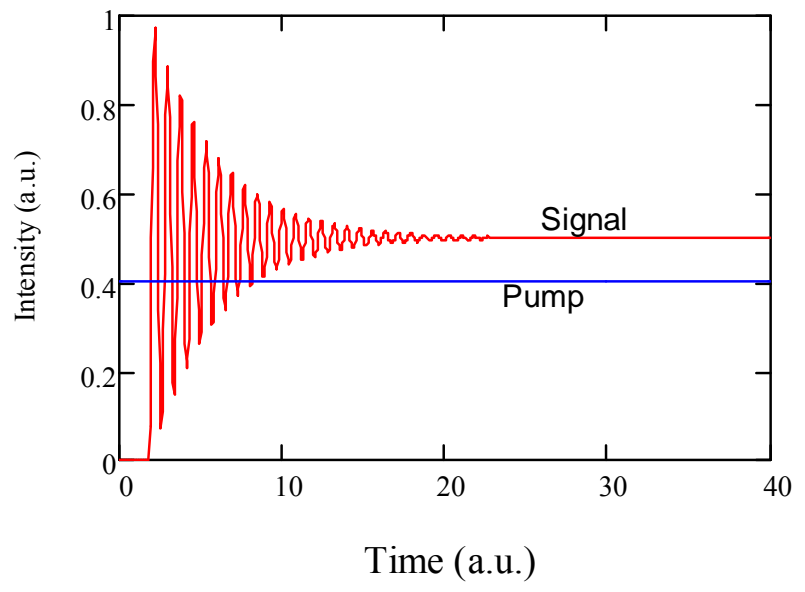

图 7.5 激光张弛振荡脉冲示意图

如果我们用方形波百浦来代替恒定的百浦, 并且方形波的下降沿正好发生在 第一个张驰振荡脉冲的地方, 那么激光输出将只有一个脉冲, 其后续的脉冲因为 泵浦的停止无法实现粒子数反转而消失。在实际情况下, 泵浦源是正弦波形而非 方波。在这样的情况下, 我们需要使第一个激光脉冲发生在百浦正弦的下降沿合 适的地方, 如果脉冲出现得太早, 那么剩下的泵浦功率能够产生足够的粒子数反 转而产生第二个脉冲甚至多个脉冲输出; 如果脉冲出现的太迟, 则说明泵浦的时 间和强度不够而不能产生高的峰值输出功率。以二极管增益开关激光器为例, 随 着提高射频百浦的重复率, 我们必须增加射频功率来保证在一个射频周期内有足 够的能量来实现反转; 但功率也不能高到会产生多脉冲。同时我们可以调节偏压 来调节第一个脉冲发生的时间以保证高功率的单脉冲输出。所以, 一般来讲, 随 着射频频率的增加, 粒子数反转的峰值增加从而能产生更强及更窄的激光脉冲。 因为泵浦的射频频率可以很容易调节, 因此激光脉冲的重复率也可以很灵活地变 
化，这是锁模激光及其他脉冲激光技术所不能做到的[115]。

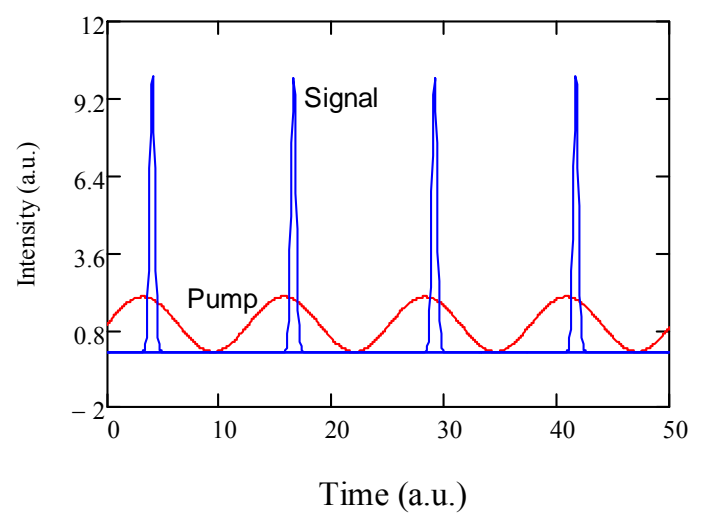

图 7.6 增益开关脉冲技术示意图

一般来讲, 增益开关固体激光器产生的脉冲长度长达几十上百 $\mathrm{ns}, \mathrm{Q}$ 开关和 倾腔激光器能产生短一点 ( ns) 的脉冲, 锁模激光可以产生更短的脉冲（短至 飞秒至亚飞秒)。但是二极管激光器的光腔可以做的非常短 ( $\mathrm{mm})$, 因此增益 开关二极管激光器可以产生皮秒量级的脉冲输出[116]。在以上所介绍的各种脉 冲激光技术中, 只有增益开关二极管激光和锁模激光能产生皮秒脉冲激光。相比 较, 锁模激光结构上更加复杂、调节困难且对机械震动非常敏感; 增益开关二极 管激光则无法提供低于皮秒的激光脉冲长度, 且峰值功率相对较弱。

从前面所讨论的我们知道, 对激光系统的要求具体到激光源主要有二: 一是 激光脉冲能和电子束脉冲很好同步; 二是激光脉冲和电子束脉冲在碰撞点能很好 的重合。从这两点要求来讲, 增益开关二极管激光能更好地满足要求。首先其激 光脉冲是受射频信号的驱动而产生的, 频率调节范围从几十 $\mathrm{MHz}$ 到几十 $\mathrm{GHz}$, 将激光驱动射频信号和加速器主脉冲信号同步非常简单; 其次, 增益开关二极管 激光在 $499 \mathrm{MHz}$ 重复率时的脉冲长度为几十个皮秒, 这将会使其与 $1 \mathrm{ps}$ 的电子 束脉冲的重合非常容易, 而且还保留着压缩激光脉冲提高峰值功率而提高康普顿 散射亮度的空间。另外, 增益开关二极管激光相对较低的输出脉冲能量并不是决 定因素, 最终脉冲功率主要取决于放大器。小巧耐用也是增益开关二极管激光器 非常重要的优势。基于这些比较, 我们确定选择增益开关二极管激光作为激光系 统的种子光源。根据作者所了解的文献记录, 增益开关二极管激光器在 Jlab 加 速器系统的应用属于首创, 而波长为 $1064 \mathrm{~nm}$ 的增益开关二极管激光器在本康普 顿激光系统的应用也是第一次[117]。 


\section{1 .5 增益开关二极管系统}

二极管激光器按结构分类主要有两种: F-P (Fabry-Perot) 二极管和分布式反 馈（Distributed Feedback，DFB）二极管。比较而言，F-P二极管价格便宜、速度 慢[118]；DFB二极管具有窄谱、快速和高信噪比等优点[119], 但是价格昂贵。 下面是我所使用的增益开关二极管激光器的示意图。该激光器需要的辅助设备 有: 用于驱动二极管的射频发生器加射频放大器, 用作DC输入的电流驱动器, 用于控制二极管温度的温度控制器。射频信号和直流电流信号通过Bias T（图 7 . 7 中大黑方块）的结合连接至二极管的阴极。

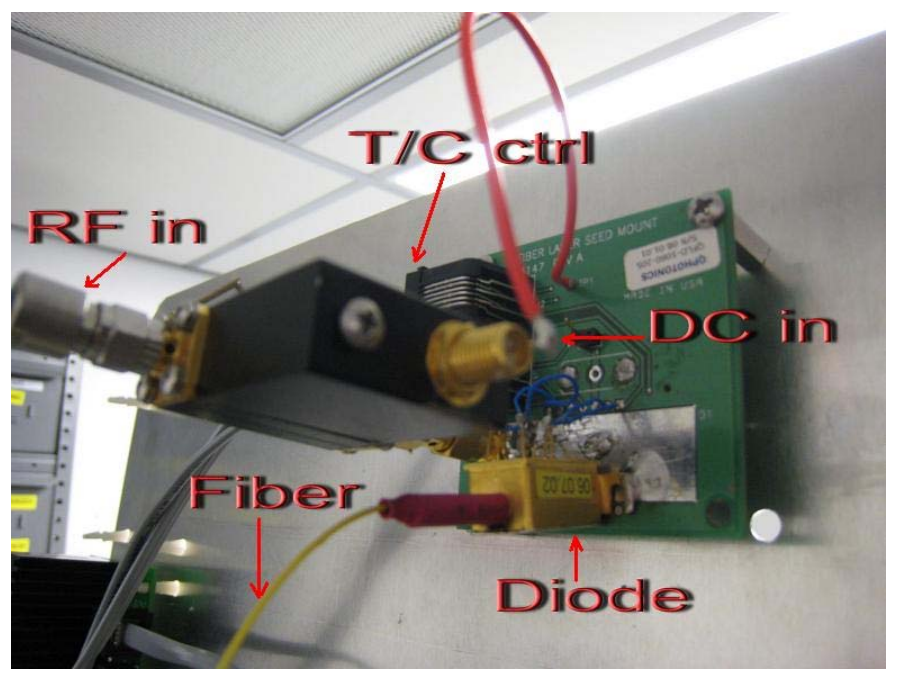

图 7.7 增益开关二极管系统

我们所使用的是 Qphotonics 公司的单模光纤耦合二极管 QFLD-1060-10S。其引 脚分布及说明如下[120]

Bottom View

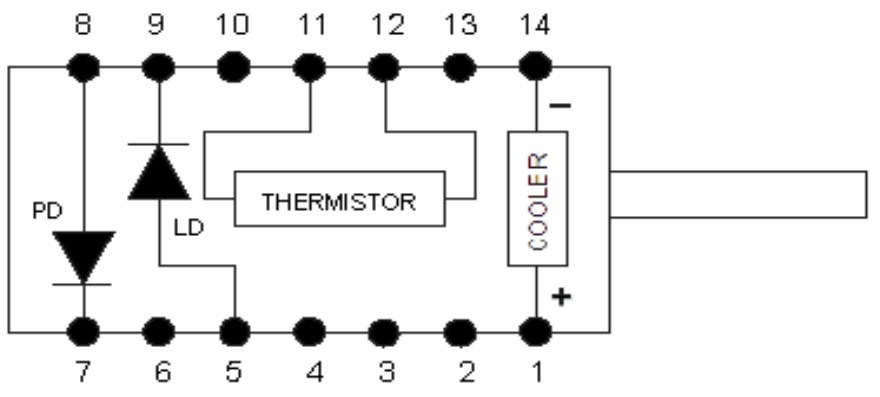

\begin{tabular}{|l|l|}
\hline Pin & Connection \\
\hline 1 & TE Cooler $(+)$ \\
\hline 5 & LD Anode $(+)$ \\
\hline 7 & PD Cathode $(+)$ \\
\hline 8 & PD Anode $(-)$ \\
\hline 9 & LD Cathode $(-)$ \\
\hline 11 & Thermistor \\
\hline 12 & Thermistor \\
\hline 14 & TE Cooler $(-)$ \\
\hline
\end{tabular}

图 7.8 二极管引脚示意图及说明

其直流驱动下的基本参数为: 
表格 7.1 二极管基本参数表

Test conditions: temperature $25^{\circ} \mathrm{C}$, power $10 \mathrm{~mW}, \mathrm{CW}$ operation

\begin{tabular}{|c|c|c|c|c|c|}
\hline $\mathbf{P}$ arameter & Symbol & Min & Typ & Max & Unit \\
\hline Optical power from pigtail & $\mathrm{P}_{\mathrm{f}}$ & 9 & 10 & 12 & mW \\
\hline Wavelength & $\lambda_{0}$ & 1055 & & 1075 & $\mathrm{~nm}$ \\
\hline Wavelength $\mathrm{v} / \mathrm{s}$ temperature coefficient & $\mathrm{d} \lambda / \mathrm{dT}$ & & 0.3 & & $\mathrm{~nm} /{ }^{\circ} \mathrm{C}$ \\
\hline Spectral linewidth (FWHM) & $\Delta \lambda$ & & 2.0 & 3.0 & $\mathrm{~nm}$ \\
\hline Fonwand current & $I_{f}$ & & 150 & 200 & $\mathrm{~mA}$ \\
\hline Threshold current & $I_{t_{1}}$ & & 25 & 40 & $\mathrm{~mA}$ \\
\hline Fonwand voltage & $V_{f}$ & & 1.7 & 2.4 & $\mathrm{~V}$ \\
\hline Rise time in pulse mode & $t_{r}$ & & 0.5 & & ns \\
\hline Monitor current $@ V_{\mathrm{rPD}}=5 \mathrm{~V}$ & $I_{m}$ & 0.07 & & & $\mathrm{~mA}$ \\
\hline TEC current & $\mathrm{I}_{\mathrm{TEC}}$ & & & 0.5 & $\mathrm{~A}$ \\
\hline Thermistor resistance (a) $25^{\circ} \mathrm{C}$ & & 9.5 & 10.0 & 10.5 & KOhm \\
\hline Storage temperature & $T_{\text {stg }}$ & -40 & & 70 & ${ }^{\circ} \mathrm{C}$ \\
\hline Operating case temperature & $T_{0}$ & 0 & & 60 & ${ }^{\circ} \mathrm{C}$ \\
\hline PD reverse voltage & $V_{\mathrm{rPD}}$ & & & 20 & $\mathrm{~V}$ \\
\hline Lead soldering temperature @10s & - & & & 260 & ${ }^{\circ} \mathrm{C}$ \\
\hline
\end{tabular}

下面是对增益开关二极管激光源束斑参数的测量结果图。得益于单模光纤耦 合的原因, 输出光斑呈良好的对称高斯分布, 两个方向的半高全宽均为 $0.8 \mathrm{~mm}$ 。

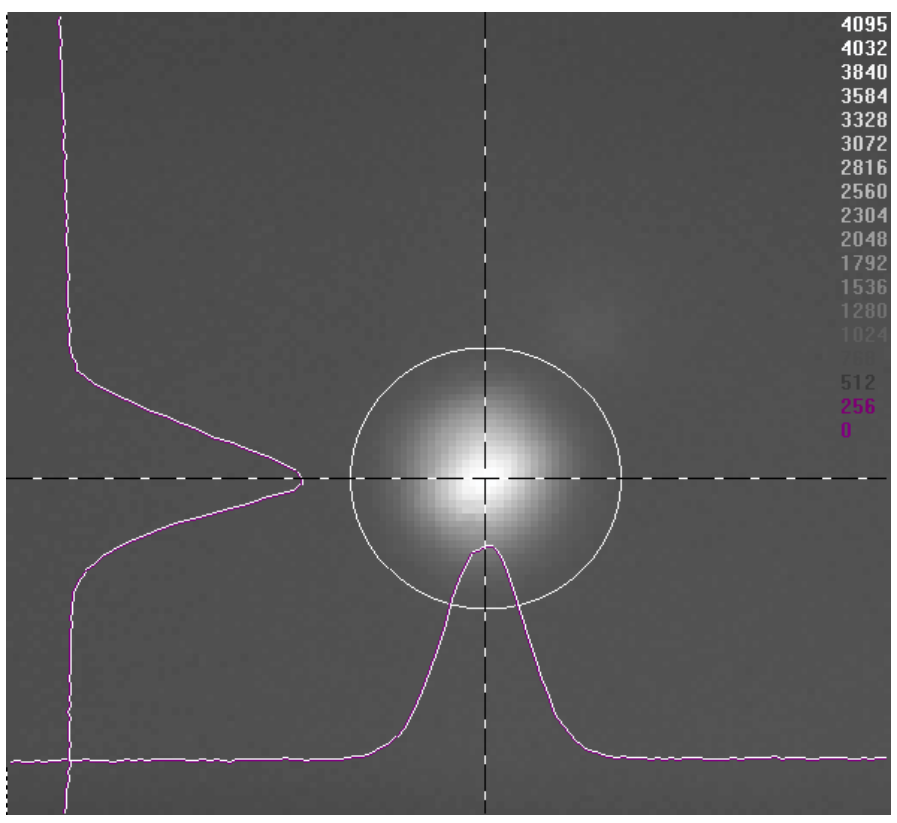

图 7.9 激光截面测量图

尽管Qphotonics公司所声称的高偏振率输出, 我还是对其进行了测量。测量 所用到的设备是：激光器+半波片 + Glan棱镜（Glan-Taylor prism）+功率计。通过 转动半波片并同时记录通过棱镜的激光功率来测量。激光的偏振度 $P=\left(P_{\max }-P_{\text {min }}\right) /\left(P_{\text {max }}+P_{\text {min }}\right)=99.2 \%$ 。 


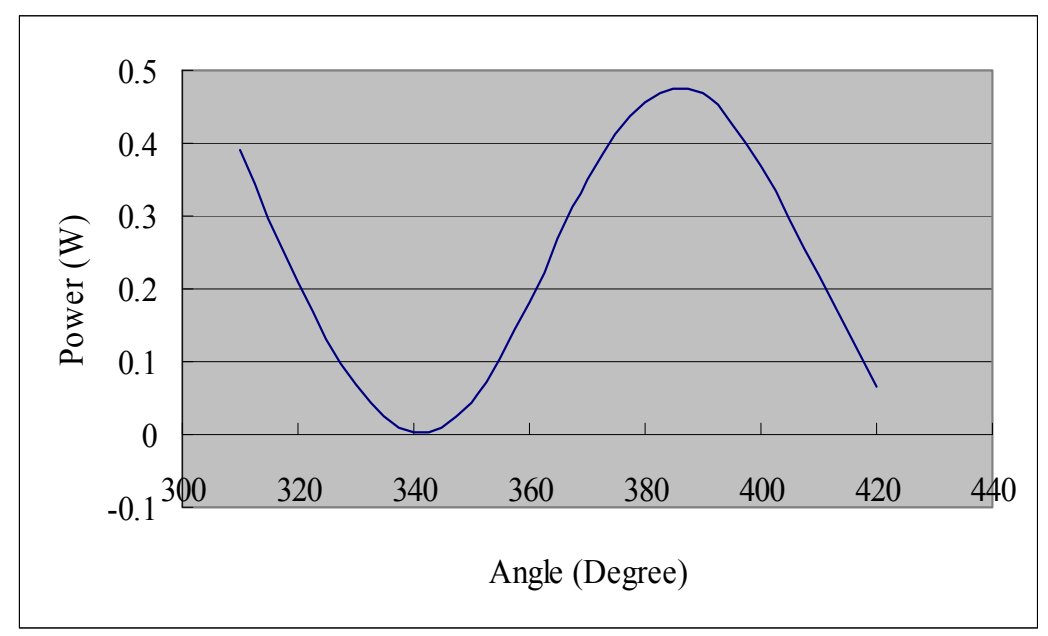

图 7.10 激光极化度测量

二极管输出的光谱形状取决于系统的多个参数。其中最重要的是半导体材料 的能量间隙 (Band Gap), 该能量间隙决定了二极管波长的一个范围。在这个范 围内的二极管光谱还是结温度、电流强度和有效折射率的函数[121]。二极管温 度的变化导致PN节能带间隙的变化, 从而引起发光光子能量的变化。通常温度 升高所导致的峰值波长的增加系数为 $0.3 \mathrm{~nm} /{ }^{\circ} \mathrm{C}$ 。驱动电流的改变会引起二极管半 导体材料折射率的变化以及二极管有效腔长的变化, 二者中有效腔长的变化占主 导地位, 因此驱动电流的增加会增加有效腔长从而导致输出波长变长。在单纵模 激光器中, 这种输出频谱的变化并非连续, 表现为纵向模式的跳跃（Model Hopping）[122]。下面是对辐射谱及中心波长与二极管温度的关系测量, 波长的 温度变化系数近似为 $0.32 \mathrm{~nm} /{ }^{\circ} \mathrm{C}$ 。

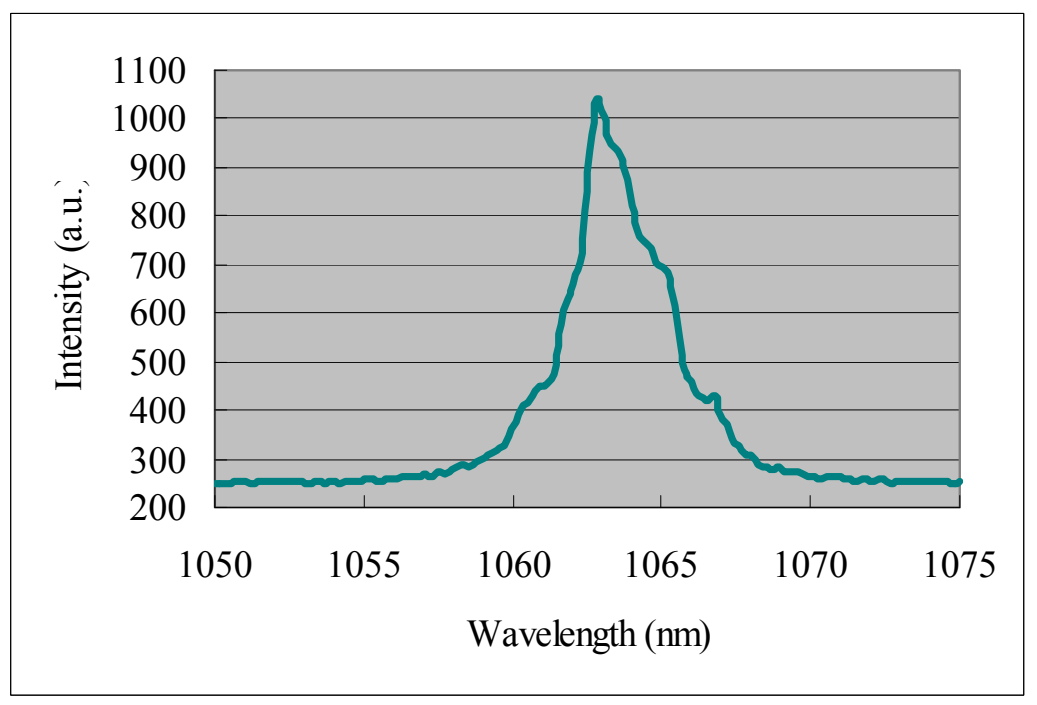

图 7.11 增益二极管输出频谱测量 


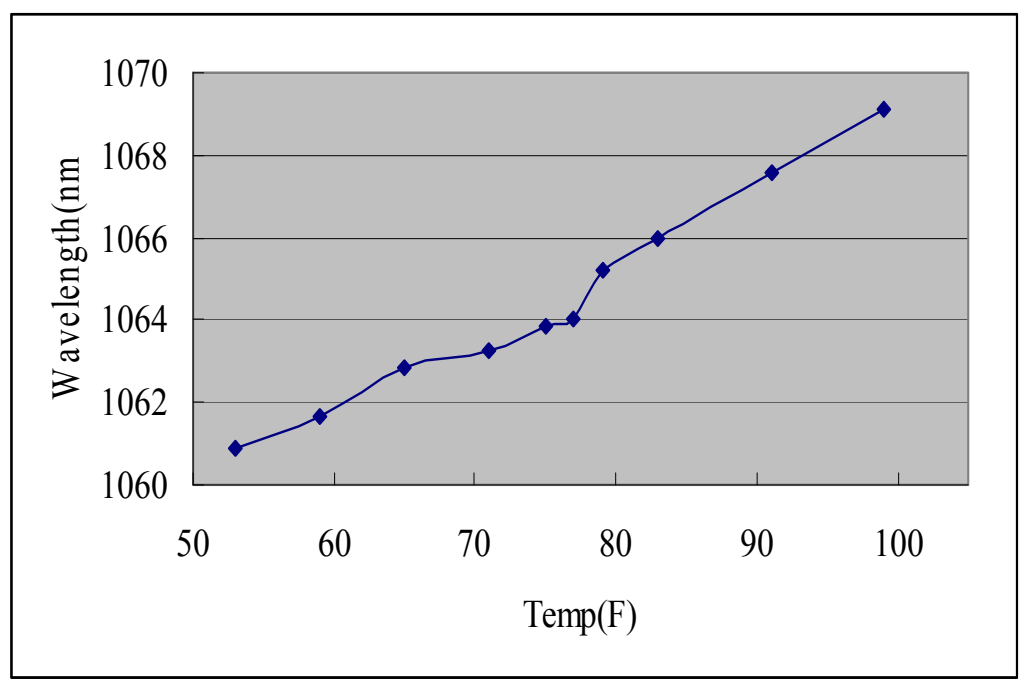

图 7.12 二极管中心波长随温度变化曲线

从后续倍频效率出发考虑, 除了激光峰值功率外最重要的就是激光源的谱 宽，超过倍频晶体谱接受度的倍频效率将会随谱宽呈指数趋势下降[123]。从图 7 . 11 可以看到, F-P二极管所发出谱的半高全宽为 $4 \mathrm{~nm}$ 。因此我们需要压缩谱宽来 提高倍频的效率。

\section{1.6 光学反馈}

光学反馈（Optical feedback）是另一种可以用来影响二极管激光输出频谱的 技术。我们知道二极管激光的增益和波长的依赖关系不强, 因此其发光波长很容 易受到外部因素的影响。通过使用一个来自波长选择元件的反馈，可以强制二极 管在反馈波长处发光。对于外部反馈元件的调节则可以达到调节最终输出的目 的。图 7.13和图 7.14中是两种常见的光学反馈设置。

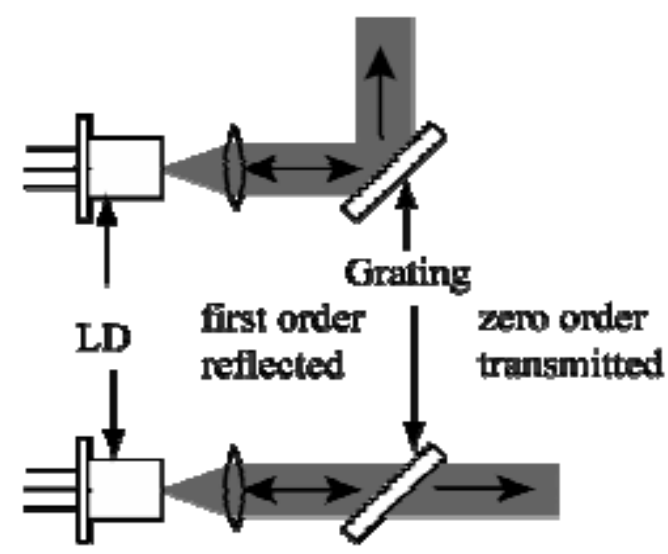

图 7.13 Littrow光学反馈示意图 


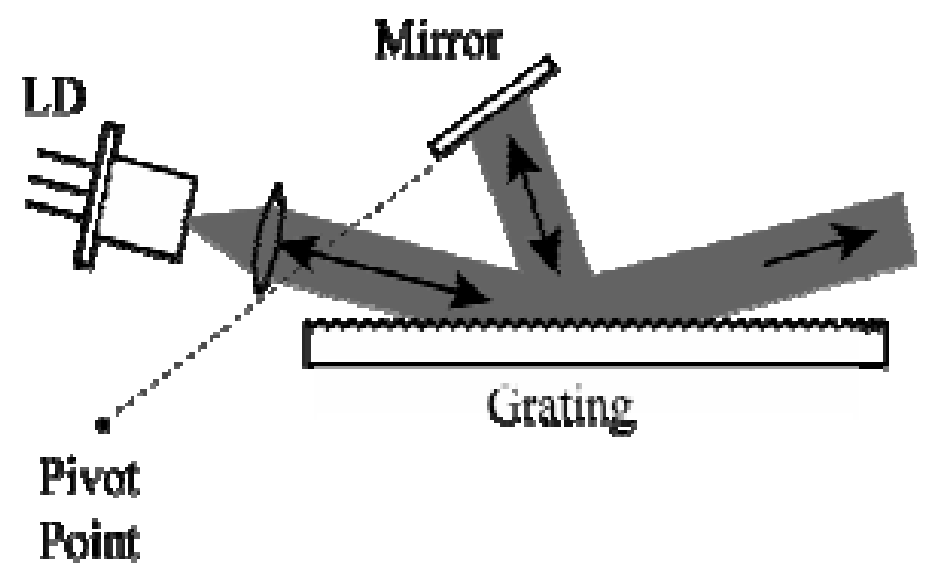

图 7.14 Littman-Metcalf光学反馈示意图

在 Littrow 设置中, 一级衍射被反射回二极管作为反馈, 零极衍射被光栅镜 面反射作为输出或者透射光栅成为主输出。在 Littman-Metcalf 设置中, 作为反 馈的一级衍射被反射镜反射回二极管, 并且反射镜和二极管-光栅所成的角度决 定了输出波长, 因此可以通过角度调节实现很宽的波长变化。

另外, Fiber Bragg Grating（FBG）也可以被利用于压缩输出谱宽甚至实现多 模激光的单模输出。FBG 是在光纤中心折射率周期变化的结构, 其作用相当于 光栅, 反射特定范围的波长而传输其他波长。这样的 FBG 结构被置入 F-P 二极 管的输出光纤中, 光纤中被 FBG 反射的窄波长范围作为反馈进入二极管, 这样 不但能稳定输出谱也能压缩二极管的输出谱宽。FBG 光纤耦合输出的 F-P 二极 管被称作 $\mathrm{FBG}$ 二极管。

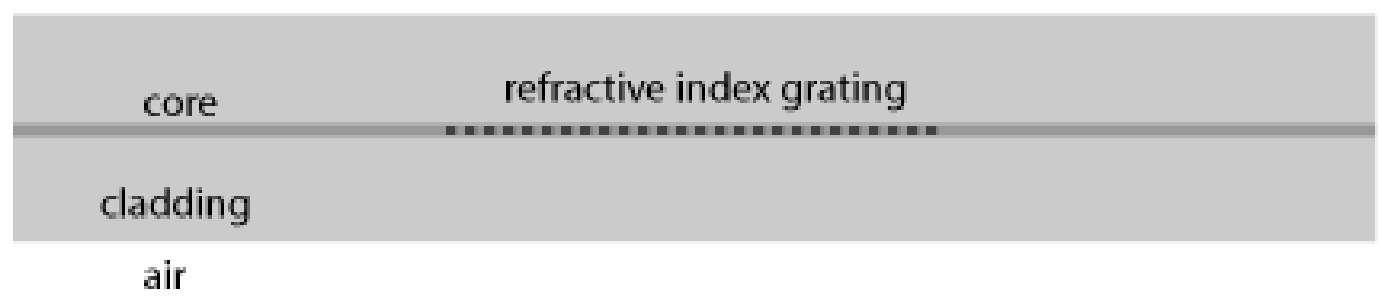

图 7.15 FBG 示意图

然而, 有别于上面所介绍的反馈方法, 我所使用的反馈结构如 图 7.16 所示。 直流驱动的 $F B G$ 二极管具有窄的谱分布, 因此利用它的输出作为反馈信号。增益 开关F-P二极管和FBG二极管之间的连接是靠 90/10 分流器来实现的。近似地, 端口 1 到端口 3 的传输效率是 $90 \%$, 端口 1 到端口 4 的传输效率是 $10 \%$ 。 


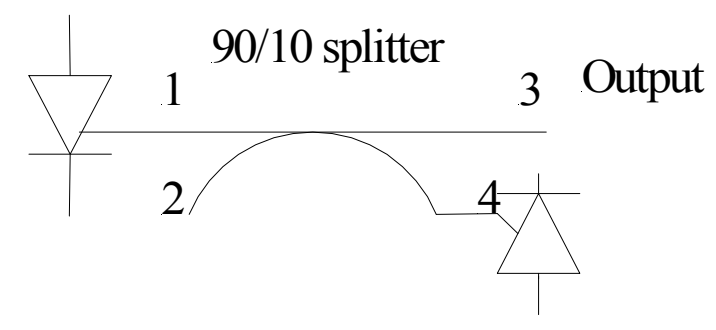

Gain switched FP

\section{CW FBG}

图 7. 16 光学反馈设置示意图

下面是在加入光学反馈前后二极管输出谱的比较

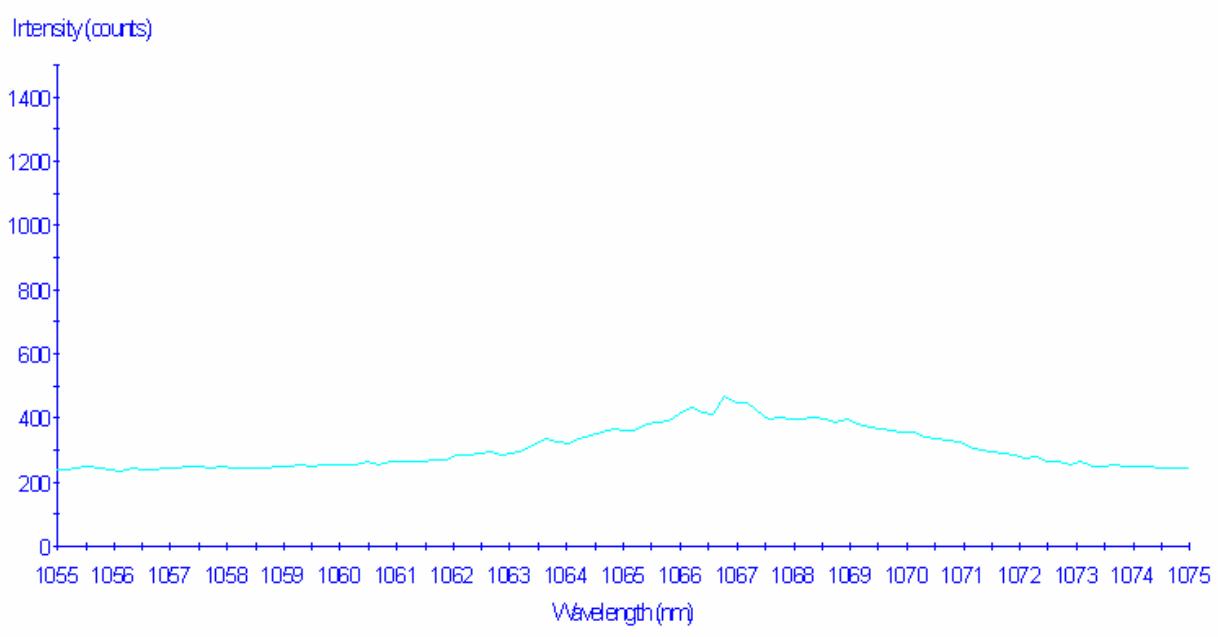

图 7.17 没有光学反馈下的频谱输出

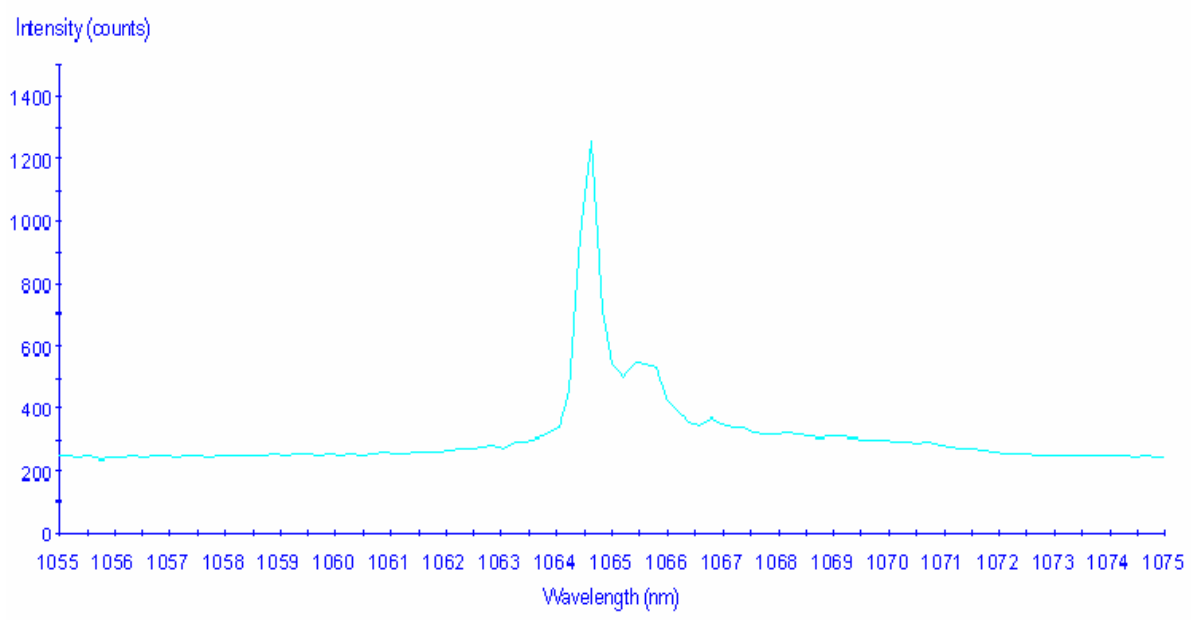

图 7.18 光学反馈下的频谱

从该光学反馈的工作原理来看, 我们不难理解对于输出波长的调节需要由 $\mathrm{FBG}$ 来实现, 而 FBG 的调节涉及到光纤内光栅结构的变化。因此, 试验中并不 能通过调节 F-P 二极管的温度来调节输出频谱。而 FBG 光栅在输出光纤内, 所 
以也不能通过调节 $F B G$ 二极管温度来调节。

\section{1.7 脉冲选择及脉冲生成技术}

尽管我们对增益开关激光脉冲宽度与重复率的关系进行了初步的分析, 认为 随着重复率的增加脉冲宽度会变窄, 但是脉冲宽度受到多参数的影响, 其变化规 律在不同的二极管材料上表现不尽相同。下面是对不同重复率下增益开关激光功 率和脉冲宽度的测量结果。

表格 7.2 不同重复率下二极管输出参数测量

\begin{tabular}{cccc}
\hline Rep. rate $(\mathrm{MHz})$ & Power $(\mathrm{mW})$ & $\mathrm{T}(\mathrm{ps}, \mathrm{FWHM})$ & $\sigma \quad(\mathrm{ps})$ \\
\hline 125 & 0.108 & 60.230 & 25.630 \\
250 & 0.224 & 53.956 & 22.960 \\
300 & 0.265 & 51.446 & 21.892 \\
400 & 0.464 & 50.192 & 21.358 \\
499 & 0.738 & 46.427 & 19.756 \\
600 & 1.176 & 46.427 & 19.756 \\
700 & 1.318 & 46.427 & 19.756 \\
\hline
\end{tabular}

其中对脉冲宽度的测量使用了 Femtochrome 公司的自相关仪 FR-103XL，该 自相关仪的测量结果受激光功率以及机械调节的影响比较大。但是, 我们还是可 以很容易看到我们所预期的变化规律。在不考虑脉冲长度压缩时, 我们希望能得 到高重复率下的短脉冲。而考虑到后续放大器及倍频晶体的性质, 恒定的平均功 率和高的重复率意味着脉冲峰值功率很低, 导致不能得到良好的倍频效率。另外, 从输出功率考虑我们希望二极管工作在高重复率的条件下, 足够的输入功率能保 证后续放大器的正常工作。综合考虑, 我们需要一种降低脉冲重复率的技术, 这 样就可以即保持短的脉冲宽度又获得低的重复率。如, 工作在 $499 \mathrm{MHz}$ 的增益激 光的重复率被减至 $125 \mathrm{MHz}$, 输出功率下降至原来的 $1 / 4$, 但还大于工作在 $125 \mathrm{MHz}$ 的增益激光的输出功率。

在激光在加速器领域的应用中, 通常会需要灵活改变激光的重复率或宏脉冲 的时间结构, 一般是在高重复率的激光脉冲序列中选取需要的脉冲。这样的任务 需要脉冲选择器来完成, 常用的脉冲选择器有电光调制器和声光调制器。在电光 
调制器中, 电信号控制的普克尔斯盒改变激光的偏振性质, 并由检波片来决定激 光脉冲的去留。而在声光调制器中, 电信号的驱动导致调制器中超声波的产生, 从而通过控制脉冲行进方向来取舍。两者的共同点在于, 调制器所需的速度是受 脉冲时间间隔而不是脉冲长度所决定的。对于 $499 \mathrm{MHz}$ 的增益开关二极管激光脉 冲，还没有具有合适的上升及下降时间的驱动信号。因此，配合所使用的光纤耦 合声光调制器, 我使用了Step Recovery Diode (SRD) 来产生合适的驱动信号, 图 7. 19 是激光脉冲选择的总体实验图

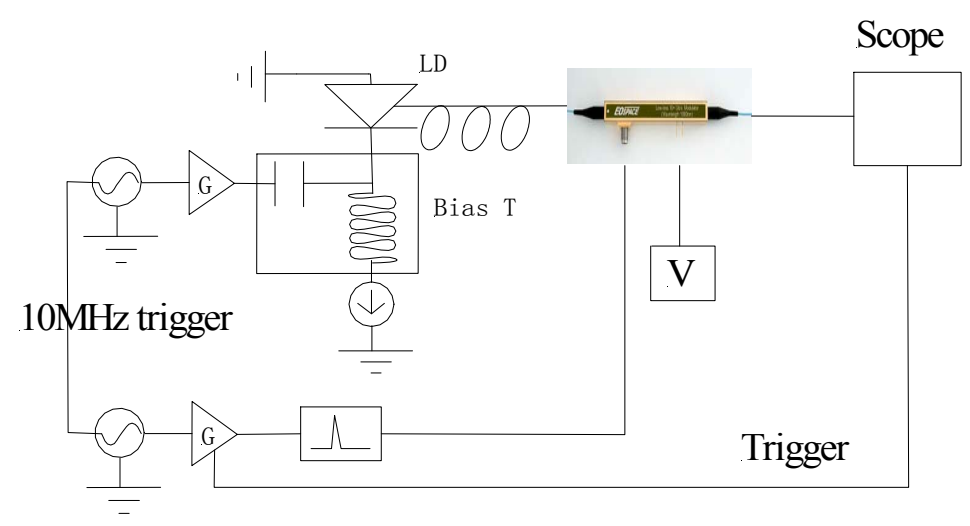

图 7.19 脉冲选择装置示意图

两台射频发生器分别用作增益开关二极管和声光调制器的驱动信号, 二者被 $10 \mathrm{MHz}$ 触发信号所同步。二极管驱动射频信号工作在高重复率（250MHz）下， 图中下方的发生器工作在目标重复率（50MHz）下。用于调制器的射频信号放大 后进入 SRD 而产生窄脉冲波形 (如图 7. 20 粉色曲线), 耦合到调制器 RF 输入 端口。输出电压范围 0-10V 的电源被接入调制器的 DC 输入端。图 7.20 中绿色 脉冲序列则是经过选择后的激光脉冲时间分布。

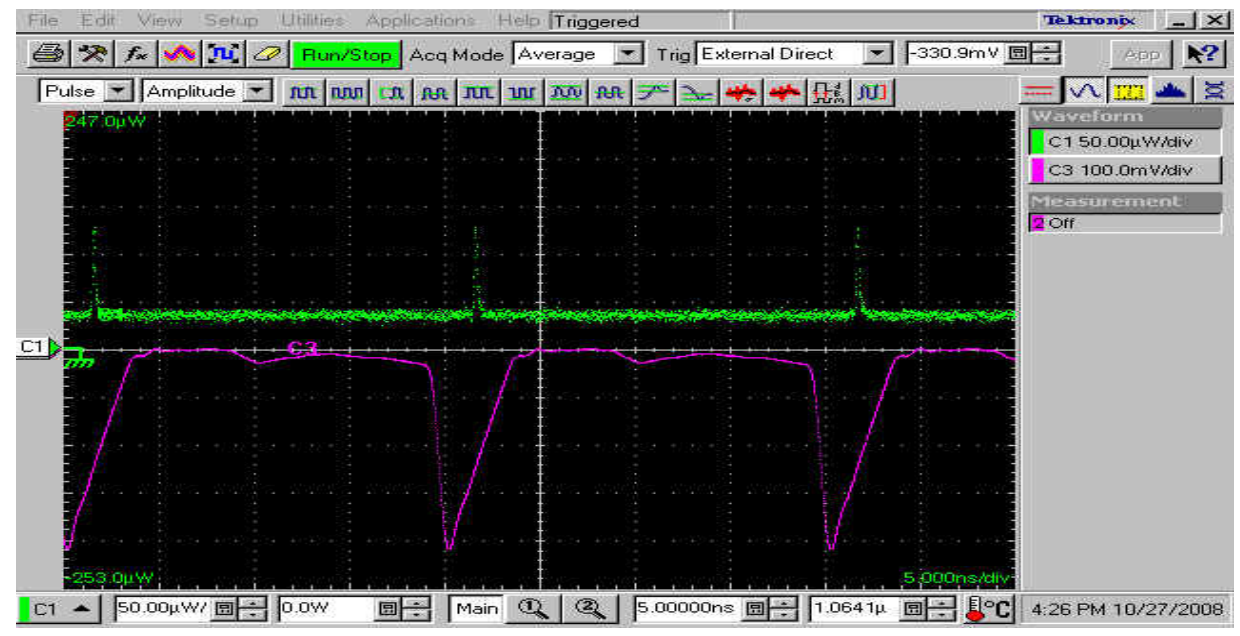

图 7.20 脉冲选择结果 
我们所使用的声光调制器及脉冲选择技术来源于电信行业的发展, 其在光纤 激光器中的成功应用还没有文献报道。而利用 SRD 实现对高重复率激光脉冲的 选择, 更是解决了脉冲快速选择的实际技术问题。利用相似的思路, 我还成功演 示了利用调制器来产生高重复率激光脉冲的技术。

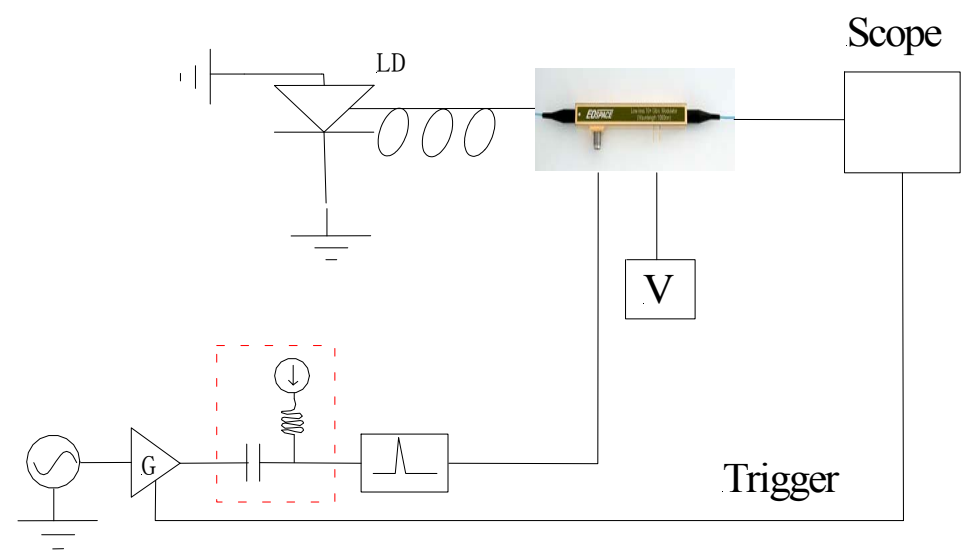

图 7.21 脉冲生成装置示意图

与之前脉冲选择不同, 二极管工作在连续模式下。SRD 之前的 Bias T 设置的 作用是调节 SRD 输出脉冲的波形, 然后该射频脉冲输入调制器, 其作用相当于 开关门：有脉冲时激光可以通过调制器，没有脉冲的时候激光被阻挡。

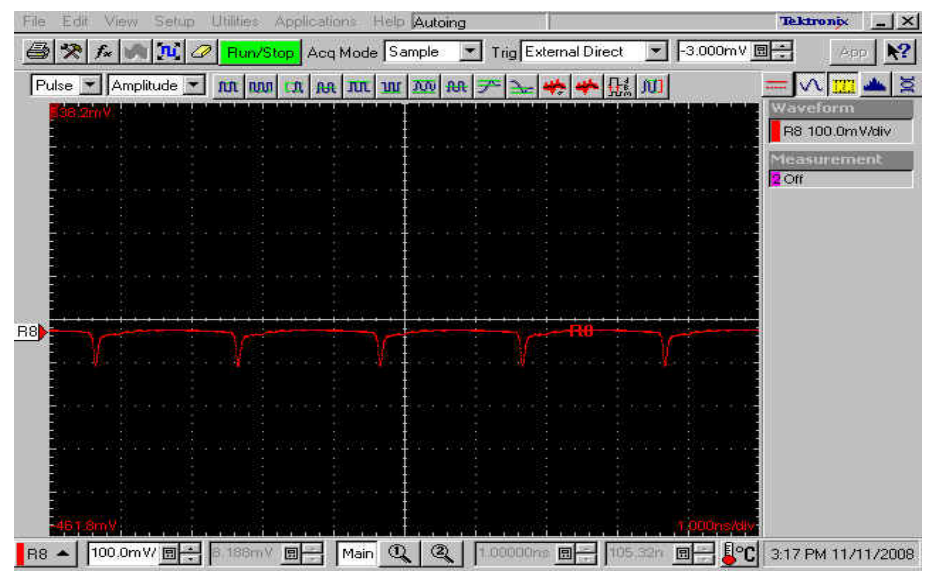

图 7.22 用于脉冲生成的射频脉冲

工作在连续模式下的激光二极管具有很好的频率特性, 能实现单模式激光输 出。这一特性在上面的脉冲生成过程中被保留, 其输出有比增益开关二极管输出 更好的相干特性。实际上, 我们所演示的是一项新的脉冲激光技术, 具备增益二 极管激光一样的可调重复率, 以及上面所说的更好的频率相干性。在需要强功率 激光的应用中，F-P腔通常被用于提高激光强度，该技术已被成功应用于连续激 光和锁模激光。基于我所做的实验, 增益开关二极管激光无法使F-P腔达到共振 
状态。究其原因是增益开关二极管的有效腔长受到多种因素的影响不稳定, F-P 腔长设置无法实时与之匹配。在保证温度以及电流稳定的条件下, 上面演示的脉 冲产生技术会产生具有更好纵向相干的激光输出, 其性质与锁模激光可以相比 拟, 有潜力解决与F-P腔匹配的问题。图 7.22 中是用于驱动调制器的脉冲序列, 图 7.23 是生成的激光脉冲序列。

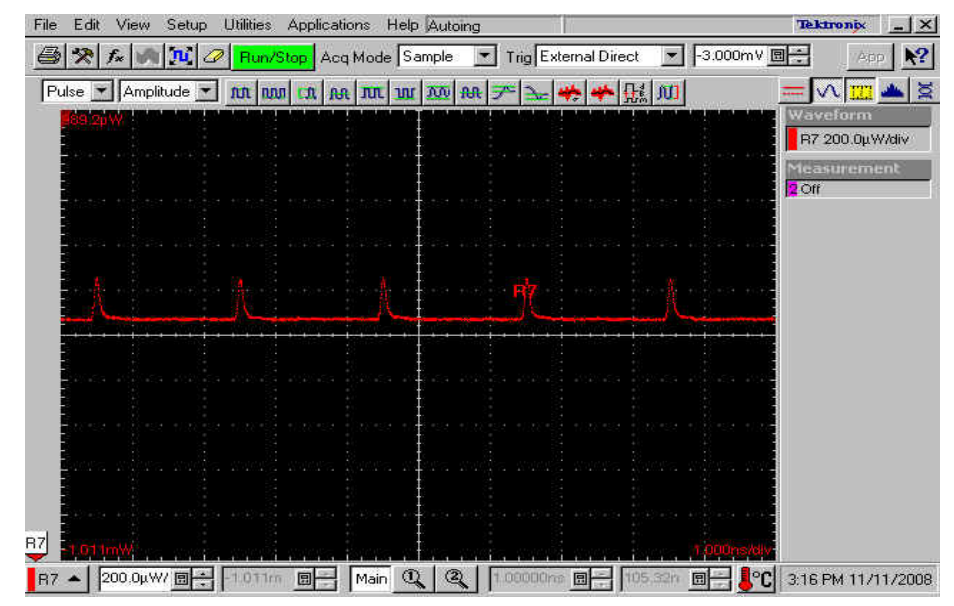

图 7.23 生成的 $500 \mathrm{MHz}$ 激光脉冲序列

\section{2 光学放大器}

状态稳定性能优良的种子光源是整个激光系统的重中之重, 也是后续放大器 稳定工作的保证。作为总结, 下面是激光源参数列表。

表格 7.3 增益激光源参数

\begin{tabular}{|c|c|}
\hline Parameter of seed & Values \\
\hline Power & $\sim 1 \mathrm{~mW}$ \\
\hline Wavelength & $1.064 \mathrm{um}$ \\
\hline Pulse width & 20ps RMS \\
\hline Beam quality & $\mathrm{M}^{2} \sim 1$ \\
\hline Beam waist & $0.4 \mathrm{~mm} 1 / \mathrm{e} 2$ radius \\
\hline
\end{tabular}

表中除了头两项外, 其他参数已达到甚至超出了我们对于激光性能的要求。 接下来的工作就是在尽量保持激光性能的同时, 提升激光功率和对激光进行倍 频。鉴于倍频对于激光功率的要求, 通常激光功率放大在倍频之前进行。在可选 择的晶体放大器、半导体放大器及光纤激光放大器中, 我选择使用了光纤激光放 大器, 主要原因在于光纤激光放大器能很好地保持激光品质。 


\subsection{1 光纤放大器}

光纤放大器是利用光纤作增益介质的光学放大器。增益介质一般是掺杂稀有 元素离子的玻璃纤维。信号光在纤维中与泵浦光共同传播的同时被放大。常用的 有工作在 1.56um的EDFA（Erbium-doped Fiber Amplifier), 工作在 1.3um的PDFA (Praseodymium-doped Fiber Amplifier) 以及工作在 1um下的YFA（Ytterbium Fiber Amplifier)。EDFA示意图如 图 7.24 所示。

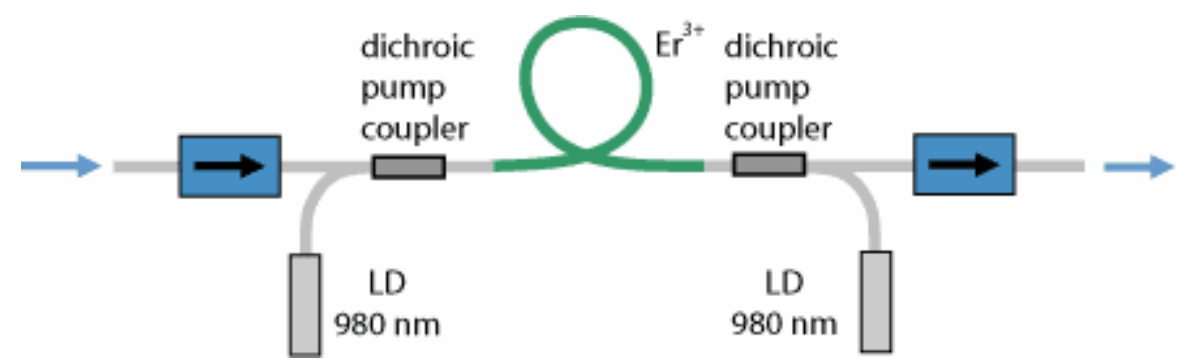

图 7.24 光纤放大器示意图

得益于光纤的细长结构, 光纤放大器在泵浦光功率不高的情况下也能实现高 的光学增益。我们选择了IPG公司生产的YFA（Ytterbium Fiber Amplifier）来放 大 $1064 \mathrm{~nm}$ 的增益开关激光源。其基本优点在于: 单模光纤耦合, 不需要水冷装 置及对输入光功率不敏感。

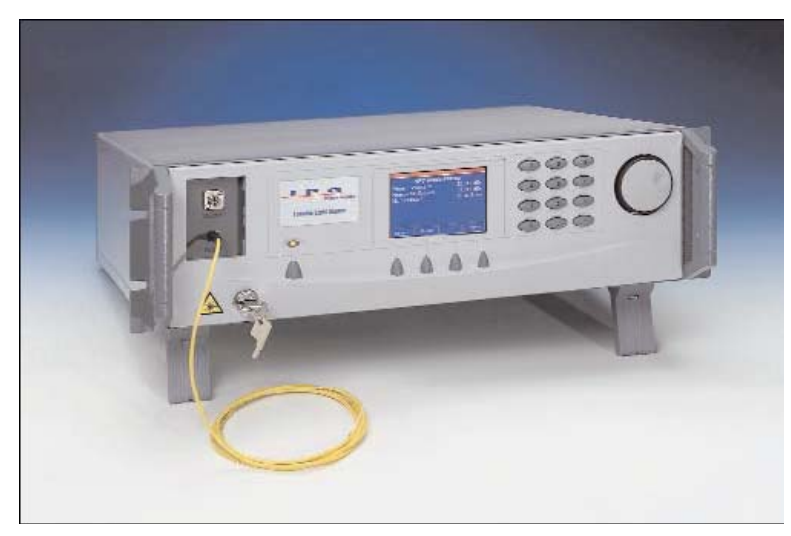

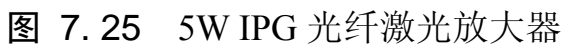

增益开关激光源输出光纤与放大器输入光纤的连接是靠光纤连接器适配器 来实现的, 关于光纤连接器的类型介绍以及适用于不同连接器的适配器的基本知 识请参考 [124]。与其他类型放大器不同的是, 光纤放大器在输入功率满足要求 的情况下, 输出功率基本恒定, 如表格 7.4中输出功率的稳定性为 $2 \%$ 。因此, 利用前面所介绍的脉冲选择技术来降低重复率, 提高峰值功率才切实可行而且相 当重要。 
表格 7.4 IPG 光纤激光放大器参数表

\begin{tabular}{lll}
\hline Parameters & Unit & YAR-5K-LP-SF \\
\hline Polarization of output signal & & linear \\
Operating wavelength range & $\mathrm{nm}$ & $1050-1120$ \\
Operating bandwidth (FWHM) & $\mathrm{nm}$ & 20 \\
Central operating wavelength & $\mathrm{nm}$ & 1064 \\
Input power range & $\mathrm{mW}$ & $1-30$ \\
Minimum input signal linewidth & $\mathrm{MHz}$ & 0.01 \\
Saturated output power (PIN=3 mW) & $\mathrm{W}$ & 5 \\
Output power tunability & $\%$ & $10-100$ \\
Output power stability (over 8 hrs) (ACC) & $\%$ & 2 \\
Relative residual pump at input/output ports & $\mathrm{dB}$ & -30 \\
Maximum power consumption (at $\left.20^{\circ} \mathrm{C}\right)$ & $\mathrm{W}$ & 75 \\
\hline
\end{tabular}

\section{2.2 激光传输测量}

放大器也同样利用单模光纤输出, 在光纤的尾部配有准直的透镜, 激光被准 直后进入自由空间。激光在自由空间的传播与在光纤内的传播遵循不同的规律。 在激光光学中, 理想的TEM010模式的激光被成为高斯光束, 其功率在截面的分 布可以用高斯函数表示[125]

$$
I(r, z)=\frac{P}{\pi w^{2}(z) / 2} \exp \left(-2 \frac{r^{2}}{w^{2}(z)}\right)
$$

其中 $w(z)$ 是从光轴中心到激光强度下降到最大值的 $1 / e^{2}$ 处的距离, 该距离等于 两倍的RMS半径。对于在自由空间传播的高斯光束, 束斑大小 $w(z)$ 会在光轴上 出现一个最小值 $w_{0}$, 该处就是激光束腰。对于波长为 $\lambda$ 的激光, 离束腰距离为 $z$ 处的束斑大小可以表示为:

$$
w(z)=w_{0} \sqrt{1+\left(\frac{z}{z_{R}}\right)^{2}}
$$

其中, $z_{R}=\frac{\pi w_{0}^{2}}{\lambda}$, 被称作瑞利长度 (Rayleigh range), 其决定了激光束不出现可 观发散下所能传播的距离。 


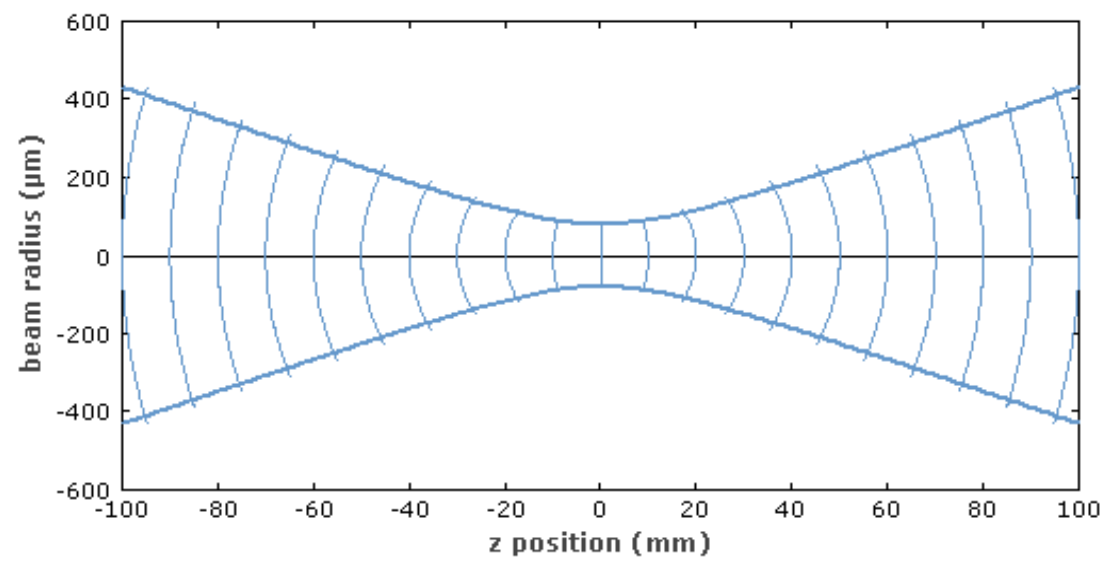

图 7.26 激光传输演化图

激光的品质由束流参数乘积（Beam Parameter Product）来描述，它是激光在 束腰处散角与束斑半径的乘积。前面所说的理想高斯光束具有最低的束流参数乘 积, $\lambda / \pi$ 。实际的激光束的束流参数乘积与理想高斯光束的束流参数乘积之比 被称作 $\mathrm{M}^{2}$ 。任意非对称激光束在自由空间的传播可以由下面公式来描述[126]

$$
\begin{aligned}
& w_{x}^{2}(z)=w_{0 x}^{2}+M_{x}^{4} *\left(\frac{\lambda}{\pi w_{0 x}}\right)^{2}\left(z-z_{0 x}\right)^{2} \\
& w_{y}^{2}(z)=w_{0 y}^{2}+M_{y}^{4} *\left(\frac{\lambda}{\pi w_{0 y}}\right)^{2}\left(z-z_{0 y}\right)^{2}
\end{aligned}
$$

其中, $\mathrm{M}_{x}^{2}, \mathrm{M}_{y}^{2}$ 是两个垂直方向上激光品质的描述参数, 其值大于 1 。基于上面 的激光传输公式, 我们可以通过测量不同纵向位置上的束斑大小来对束流品质进 行分析, 下面是对水平束斑传播测量的结果

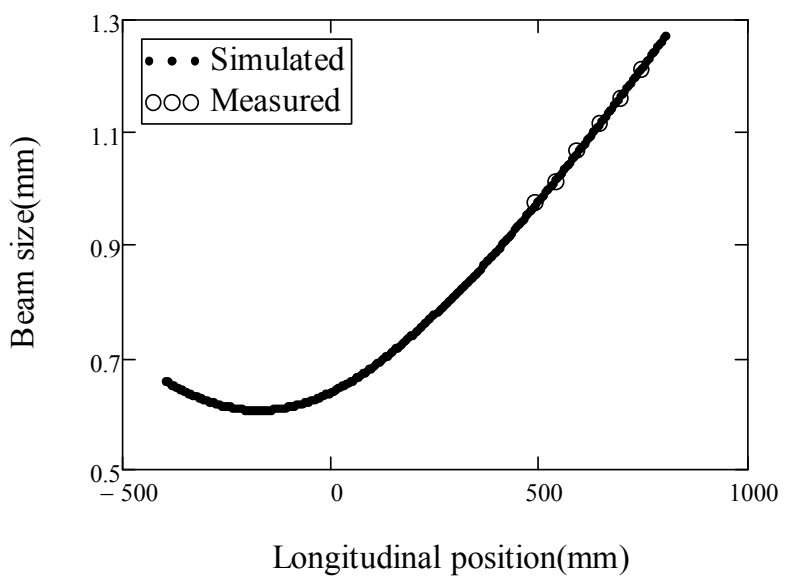

图 7.27 水平方向激光传输测量 
图中坐标原点取在放大器输出光纤的出口, 模拟计算的结果是: 水平束腰 $w_{0 x}=0.608 \mathrm{~mm}$, 水平束腰位置 $z_{0 x}=-176 \mathrm{~mm}, \mathrm{M}_{x}^{2}=1.03$ 。对于坚直方向的测量 及模拟结果如下: 坚直束腰 $w_{0 y}=0.58 \mathrm{~mm}$, 坚直束腰位置 $z_{0 y}=-180 \mathrm{~mm}$, $\mathrm{M}_{y}^{2}=1.08$ 。

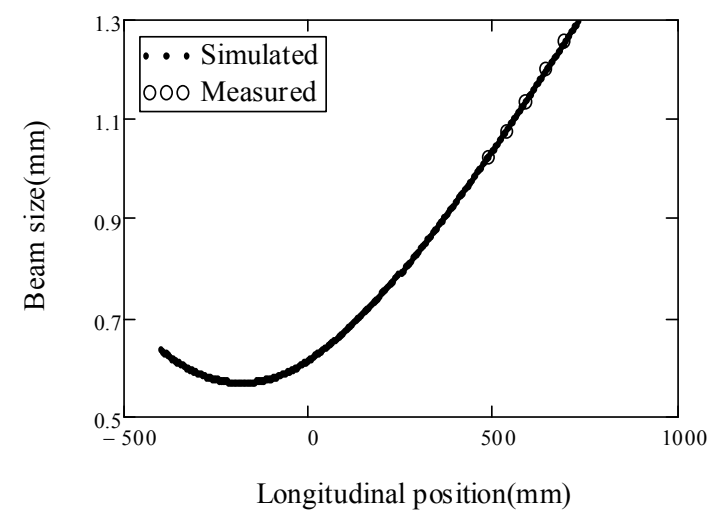

图 7.28 坚直方向激光传输测量

\section{3 二次谐波的产生}

二次谐波是非线性晶体内光学非线性过程的产物。二次谐波及高次谐波的产 生对于扩展激光波长范围具有很重要的意义。受原子能级分布所决定, 激光波长 一般分布在近红外区域。二次谐波及高次谐波是得到可见及紫外激光的必备手 段。

\section{3.1 二次谐波原理}

二次谐波产生的一个先决条件是强激光场输入，我们一般将激光场表示为：

$$
\tilde{E}(t)=E e^{-i \omega t}+\text { C.C. }
$$

因介质的二阶非线性反应激光场所激发的电极化矢量可以表示为:

$$
\tilde{P}^{(2)}(t)=2 \chi^{(2)} E E^{*}+\left(\chi^{(2)} E^{2} e^{-2 i \omega t}+\text { C.C. }\right)
$$

从极化矢量的表达式很容易看出, 频率为 $\omega$ 的单色光波输入会激发新的频率 $2 \omega$ 的产生。在量子理论中, 二次谐波的产生包含两步[127]：首先，随着对两个基 频光子的吸收, 电子从初始能级跳跃到较高能级; 然后, 随着电子回到初始能级, 
释放出具有双倍能量的光子。一般的非线性材料都是具有色散的, 所以基频和倍 频光在介质内传播速度不一样。那么在没有相位匹配的条件下, 不同点产生的二 次谐波会出现相消干涉。为了得到总的二次谐波的强度, 我们需要在考虑相位的 同时对二次谐波沿光学路径积分 [128]。出于简单考虑, 可以将基频及其激发的 极化矢量表示为

$$
\begin{aligned}
& E^{1}(x)=E^{1}(0) \cdot e^{-i k_{1} x}, \\
& P^{1}(x)=\chi \cdot E^{1}(x) \cdot E^{1}(x)=\chi \cdot E^{1}(0) \cdot E^{1}(0) \cdot e^{-i 2 k_{1} x}
\end{aligned}
$$

如前所述, 二次谐波强度应正比于极化矢量的平方,

$$
E^{2}(x)=K^{\prime} \cdot P^{2}(x)=K \cdot E^{1}(0) \cdot E^{1}(0) \cdot e^{-i 2 k_{1} x}
$$

这里, $\mathrm{K}$ 是包含非线性系数和折射率作用的常数。产生的二次谐波在晶体中以波 矢 $k_{2}$ 运动, 如果观察点在任意位置 $x^{\prime}$, 看到的二次谐波是

$$
E^{2}\left(x^{\prime}\right)=E^{2}(x) \cdot e^{-i k_{2}\left(x^{\prime}-x\right)}=K \cdot E^{1}(0) \cdot E^{1}(0) \cdot e^{-i k_{2} x^{\prime}} \cdot e^{-i\left(2 k_{1}-k_{2}\right) x}
$$

假设晶体长度为 $\mathrm{L}$, 总的二次谐波强度将是上面方程沿晶体长度的积分

$$
\begin{aligned}
E_{t}^{2}\left(x^{\prime}\right) & =K \cdot E^{1}(0) \cdot E^{1}(0) \cdot e^{-i k_{2} x^{\prime}} \int_{0}^{L} e^{-i\left(2 k_{1}-k_{2}\right) x} d x \\
& =K \cdot E^{1}(0) \cdot E^{1}(0) \cdot e^{-i k_{2} x^{\prime}} \cdot e^{i \frac{\Delta k}{2} L} \cdot \frac{\sin (\Delta k L / 2)}{\Delta k / 2}
\end{aligned}
$$

其中,

$$
\Delta k=k_{2}-2 k_{1}=\frac{2 \pi}{\lambda_{2}} n\left(\omega_{2}\right)-2 \frac{2 \pi}{\lambda_{1}} n\left(\omega_{1}\right)=\frac{4 \pi}{\lambda_{1}}\left(n\left(\omega_{2}\right)-n\left(\omega_{1}\right)\right)
$$

$\lambda_{1}$ 和 $\lambda_{2}=\lambda_{1} / 2$ 分别是基频和二次谐波在真空中的波长。二次谐波的变化依赖于 方程中的最后一项, 该项在 $\Delta k \neq 0$ 时以正弦波振荡, 在 $\Delta k \rightarrow 0$ 时趋近于最大极 限 $\mathrm{L}$ 。正弦函数从零到最大的长度, 被定义为晶体的相干长度, $L_{c}=\frac{\pi}{\Delta k}$ 。二次谐 波的强度主要取决于相位差 $\Delta k$, 通常表示成为

$$
I^{(2)}=C \cdot d_{e f f}^{2} \cdot I^{(1)^{2}} \cdot \frac{\sin ^{2}(\Delta k L / 2)}{(\Delta k / 2)^{2}}
$$

$d_{\text {eff }}$ 是晶体的有效非线性张量。在脉冲激光的情况下, 上面所使用的强度应该是 峰值强度而不再是平均强度。 
假设非耗尽条件（在低的二次谐波产生率的条件下基频强度近似保持不变） 成立，不同相位匹配下二次谐波随空间坐标的变化如下图所示

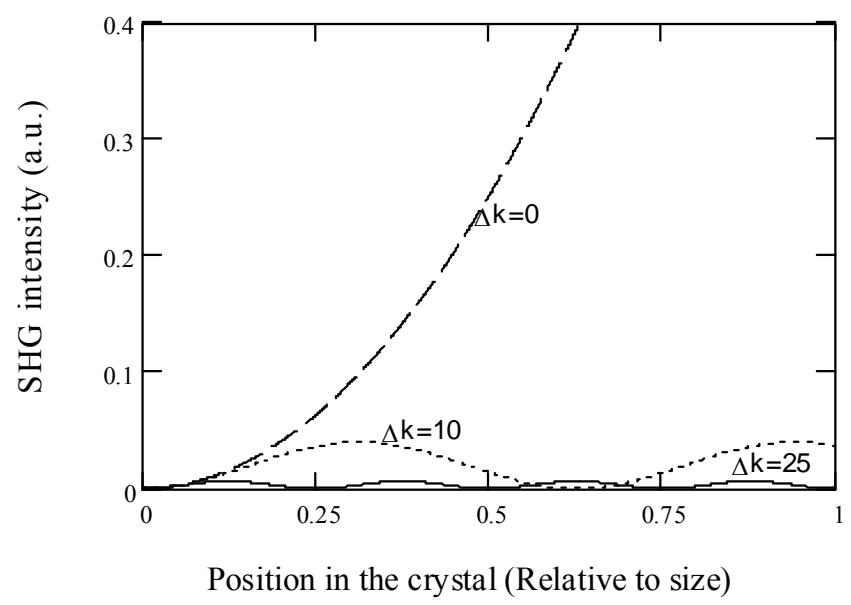

图 7.29 二次谐波强度增长与相位匹配

从公式看出, 二次谐波的强度与基波强度的平方成正比, 因此将输入光聚焦到一 个非常小的束斑能提高倍频效率。但是, 强聚焦导致瑞利长度缩短, 这样束斑在 晶体会发散得更厉害。而且, 束斑不能被压缩得小于晶体的偏移距离 $\rho L$, 其中 $\rho$ 是晶体的偏移角度，否则二次谐波和基波在晶体内分离而失去空间相干性。高斯 光束在晶体内传播如图 7.30所示

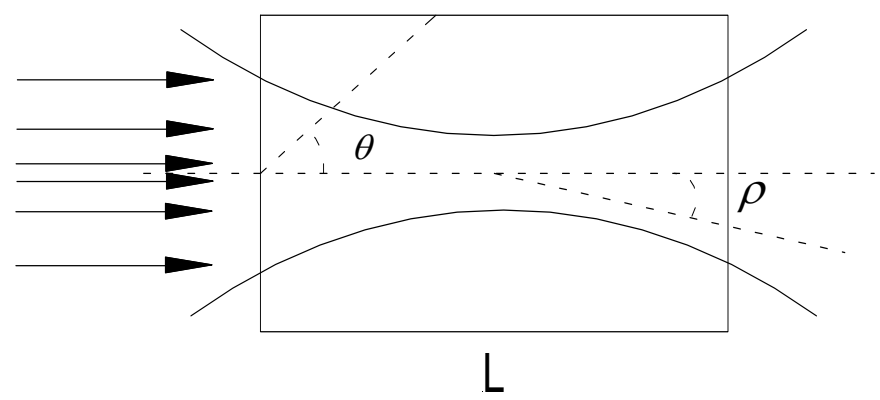

图 7.30 高斯光束入射晶体示意图

\subsection{2 倍频晶体}

适用于 $1064 \mathrm{~nm}$ 激光的倍频晶体主要有: KDP (KH2P04), BBO ( $\beta$-BaB204)， BIB0（BiB306），LB0（LiB305）。因为相对较低的非线性光学系数, KDP 是第 一个被篮选掉的晶体。下面是其它三种晶体的主要参数比较 
表格 7.5 常用红外倍频晶体参数比较

\begin{tabular}{|c|c|c|c|}
\hline Nonlinear Optical Property Comparison \\
\hline Properties & BIBO & LBO & BBO \\
\hline Length (mm) & 10.4 & 10 & 8 \\
\hline Deff (pm/V) & 3.3 & 0.81 & 2.0 \\
\hline Walk-Off (mrad) & 40.7 & 11.3 & 60.3 \\
\hline Output Power (W) & 2.8 & 1.52 & 2.1 \\
\hline Conversion Efficiency & $63 \%$ & $33 \%$ & $47 \%$ \\
\hline
\end{tabular}

相比较而言, 尽管 $\mathrm{LBO}$ 晶体的非线性系数并不是最高, 但我们最终选择了 它因为其所具有的独特优点: 在 1.0-1.3um 范围内可以实现温度控制的非临界相 位匹配，并且其有比较大的频谱接受度和很高的损伤阈值。

所谓非临界相位匹配是指, 基波及二次谐波被准直到晶体内特定方向上, 通 过调节晶体温度来消除二者的相速度差从而达到相位匹配, 基本思想是将复杂繁 琐的方向调节简化为晶体工作温度的调节 [129]。该方法的优点在于, 对于小的 角度偏离不敏感并且消除了空间偏离现象。因此, 晶体内的激光可以被聚焦到更 小的束斑, 从而达到比其他晶体更高的转换效率。然而, 非临界相位匹配所需要 的温度一般高于室温, 因此晶体需要工作在能实现温度精细控制的加热炉内, 同 时晶体表面的防反射膜必须能承受长期工作在该温度下。

非临界相位匹配 LBO 晶体工作温度与基波波长的关系是 [130]

$$
\begin{aligned}
& T_{1}=\left(-1.8933 \lambda^{4}+8.8866 \lambda^{3}-13.0198 \lambda^{2}+5.4015 \lambda^{2}+0.8639\right) \times 10^{3} \\
& T_{2}=\left(0.8781 \lambda^{4}-6.9545 \lambda^{3}+20.7342 \lambda^{2}-26.3780 \lambda^{2}+12.020\right) \times 10^{3}
\end{aligned}
$$

$T_{1}, T_{2}$ 分别是波长范围从 $0.95 \mathrm{um}$ 到 $1.2 \mathrm{um}, 1.2 \mathrm{um}$ 到 $1.8 \mathrm{um}$ 的晶体工作温度, 所用单位是摄氏度, 波长单位是微米。下面是波长 $(0.95-1.2 \mathrm{um})$ 与工作温度关 系图 


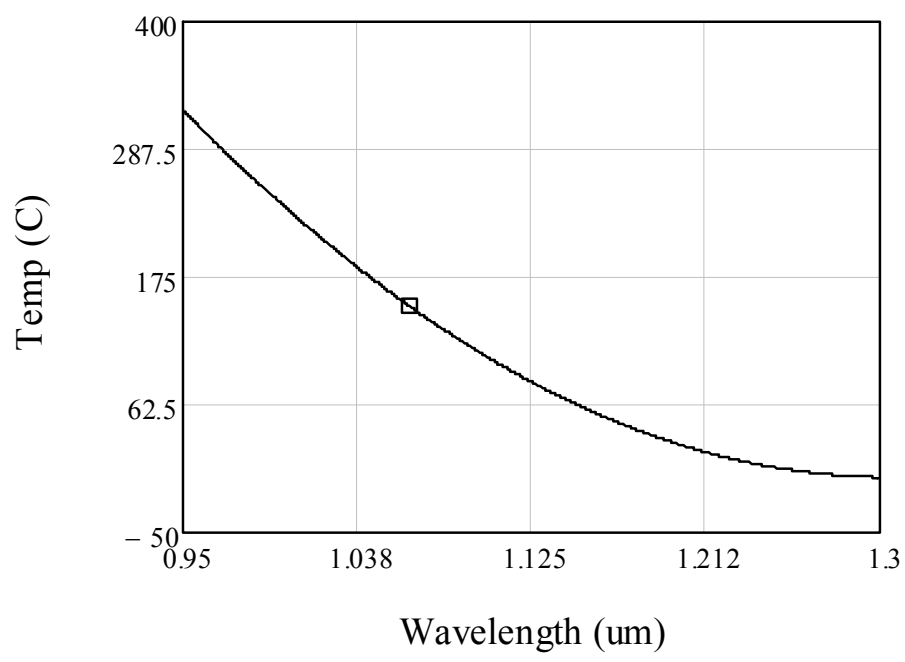

图 7.31 LBO 晶体相位匹配温度与波长关系

对于波长为 $1.064 u m$ 的激光, 晶体工作在图中小方框处, 温度约为 $149^{\circ}$ 。

\section{3.3 倍频系统及结果}

以福建福晶公司生产的 $\mathrm{LBO}$ 晶体为中心元件, 我搭建了以下倍频系统

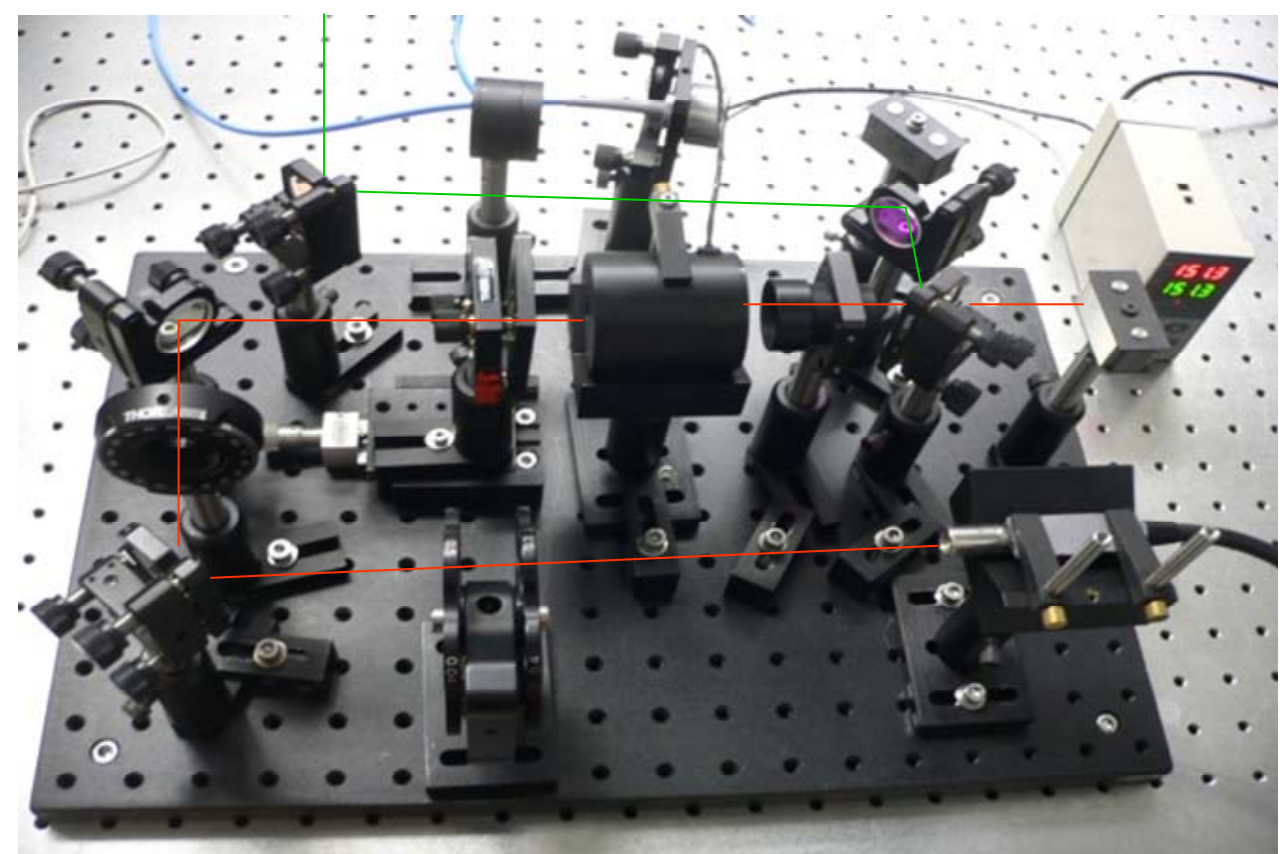

图 7.32 倍频系统实物图

光纤放大器的输出端被固定在 $\mathrm{V}$ 字架上, 下游的中性滤波片的作用是方便高功率 下的准直调节，其后的两个高反射镜之间是二分之一波片，然后就是聚焦透镜加 
LBO 晶体加透镜的组合, 从晶体出来的基频和二次谐波的混合波先后经过三个 双色镜, 最后输出比较纯的绿光, 其中基频波主要被第一个双色镜后的靶所阻挡, 右上角是 $\mathrm{LBO}$ 晶体的温度控制器。

下面是在增益开关二极管工作在不同频率下对转换效率的测量

表格 7.6 不同重复率下谐波功率测量

\begin{tabular}{cc}
\hline Rep.(MHz) & Pgreen(mW) \\
\hline 125 & 157.2 \\
200 & 145 \\
250 & 135.6 \\
300 & 136.4 \\
400 & 131.2 \\
500 & 123.5 \\
600 & 120 \\
700 & 135 \\
\hline
\end{tabular}

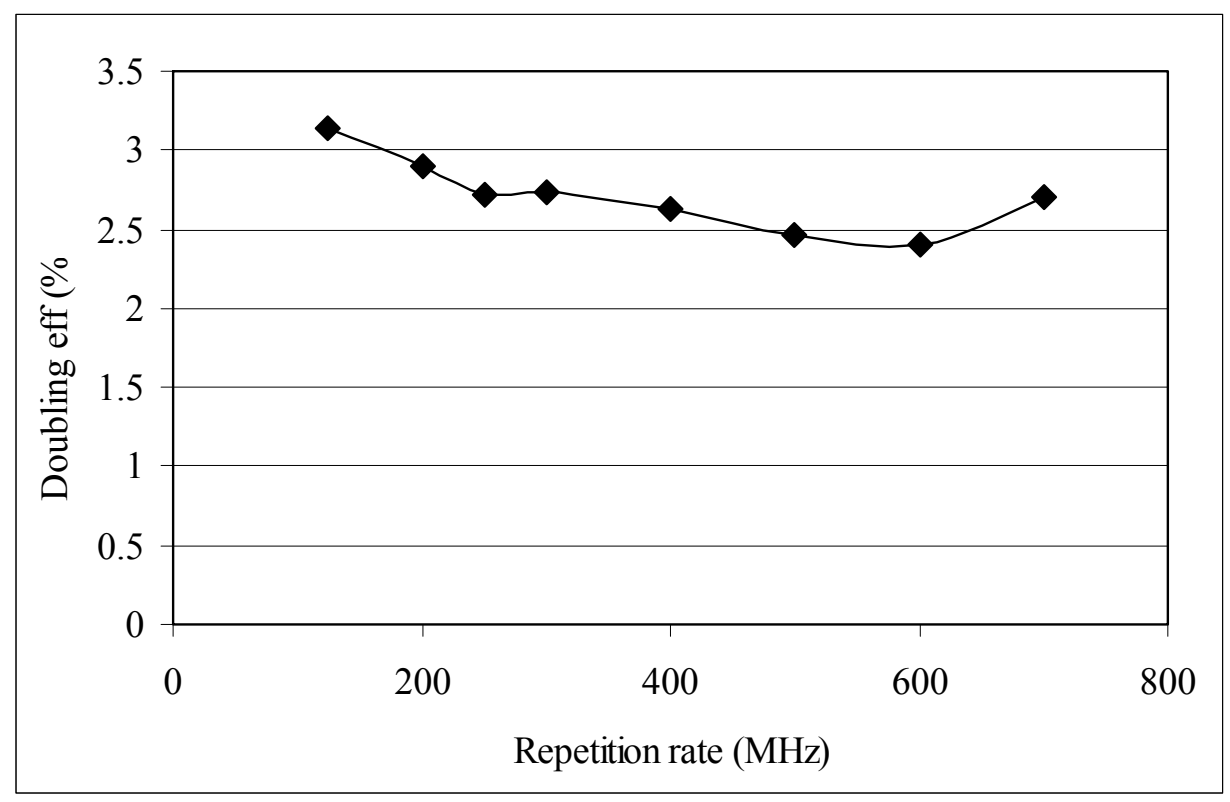

图 7.33 不同重复率下转换效率测量

同时, 在 $500 \mathrm{MHz}$ 工作频率下对增益开关二极管的工作温度也进行了优化测量 


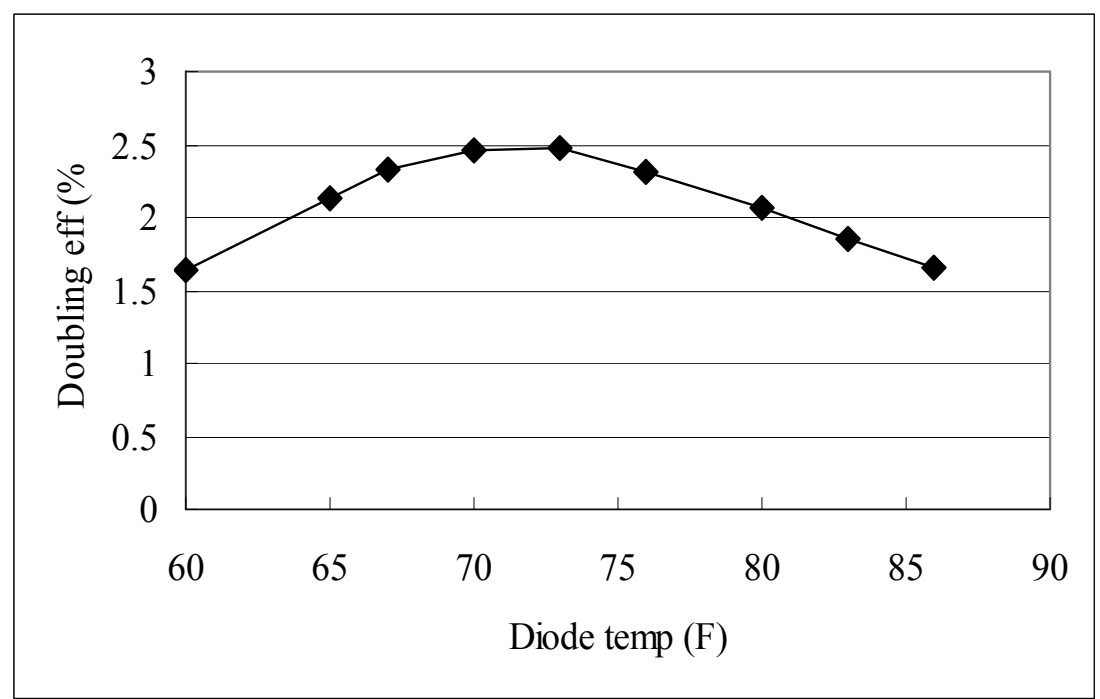

图 7.34 二极管温度对转换效率的影响

以上所得到的二次谐波转换效率 (2-3\%) 以及二次谐波绿光功率 (约 $130 \mathrm{~mW}$ ) 是无法满足工作需要的。在放大器功率上升到 $50 \mathrm{~W}$ 后, 转换效率会有一个非线 性的上升, 我们的期望值是转换效率能超过 $20 \%$, 得到超过 $10 \mathrm{~W}$ 的绿光功率。 在进行相应功率升级之前, 我进行了相关转换效率的计算, 一方面是要验证已得 到的实验结果, 另一方面是对转换效率随功率的变化进行预测。

\subsection{4 转换效率模拟}

所进行的模拟计算中我使用到了软件 SNLO $[131,132]$, 它是一款帮助选择非 线性晶体并计算预测其性能的免费软件。

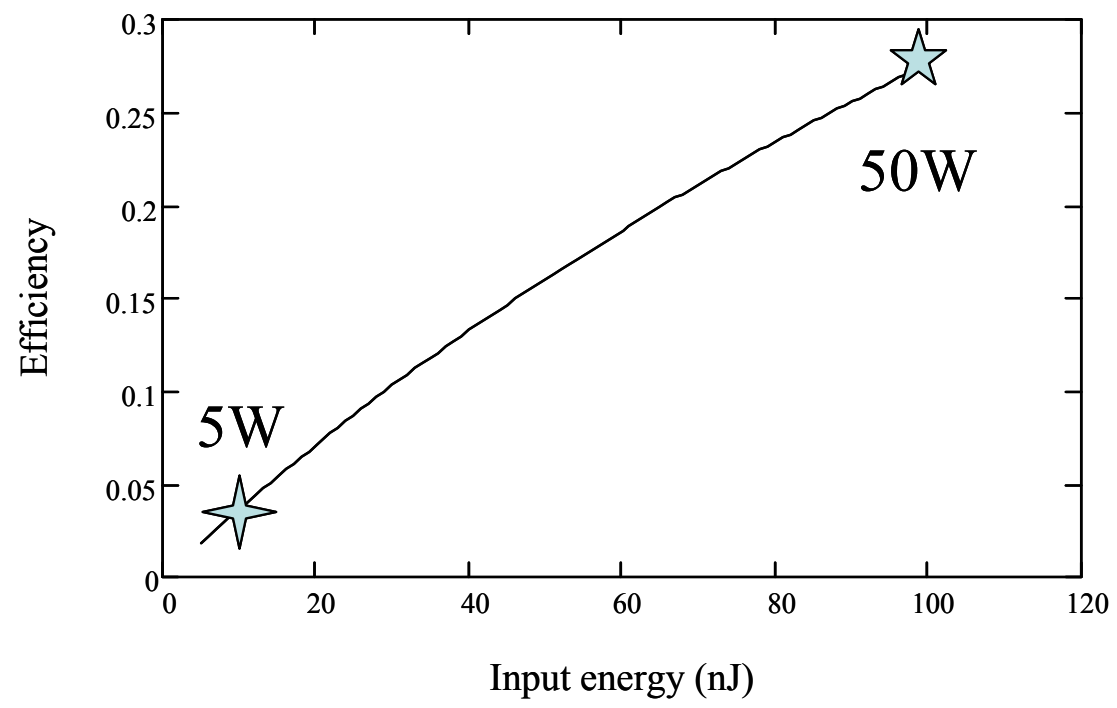

图 7.35 基于 SNLO 的转换效率的模拟 
基于 [133] 中所发展的数值计算公式，我也进行了类似的计算进行进一步的验证

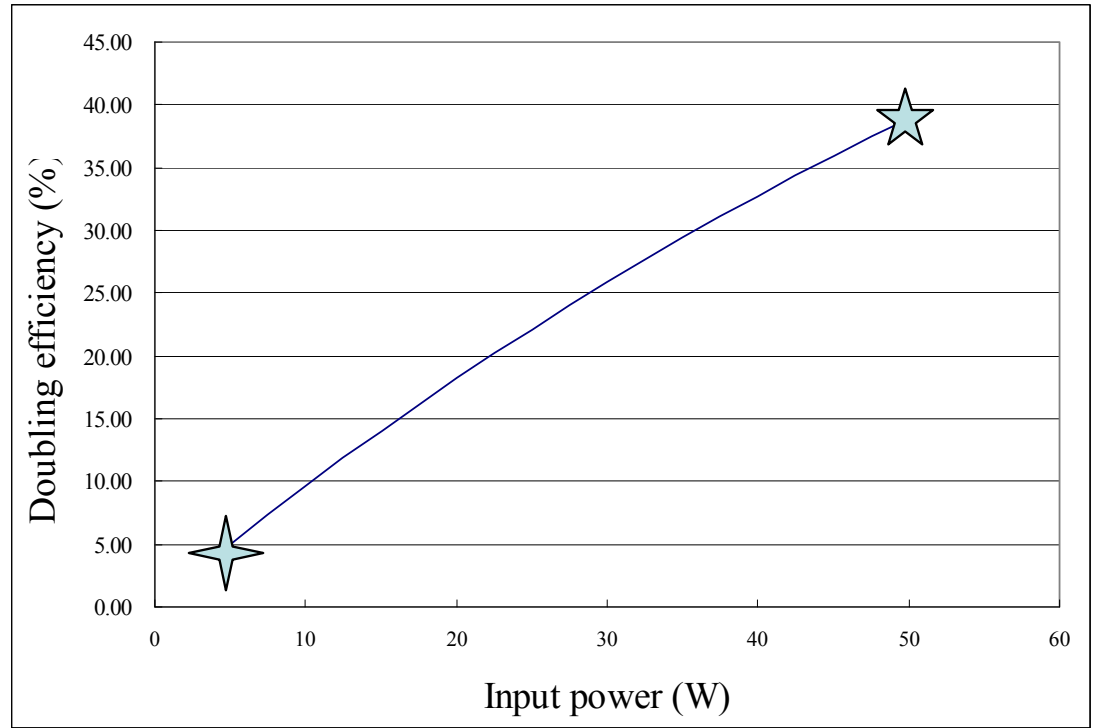

图 7.36 转换效率的数值计算

二者模拟的结果基本符合, 差异主要来自于数值模型的非耗尽区假设。在转 换效率比较低时, 二次谐波的产生并没有消耗太多的基波功率, 近似非耗尽条件, 因此二者结果符合得更好; 当转换效率上升, 不能再忽略因二次谐波的产生消耗 的基频功率，这时数值模型高估了 $\mathrm{LBO}$ 晶体能达到的转化效率。

在 $5 \mathrm{~W}$ 输出功率、 $500 \mathrm{MHz}$ 工作频率的条件下, 最高二次谐波功率和最佳的 转换效率分别为 $170 \mathrm{~mW}$ 和 $3.4 \%$, 此时晶体温度、激光聚焦、二极管温度及驱动 电流等都经过了仔细的优化。两种模型在 $5 \mathrm{~W}$ 时的结果和实验结果都符合得很 好, 因此, 在保持激光品质的前提下, 随着放大功率增加到 $50 \mathrm{~W}$, 我们相信能 得到大于 $20 \%$ 的转换效率。

\section{4 放大器展望}

因为无法承受高功率，半导体激光放大器不适合工作在 $5 \mathrm{~W}$ 到 $50 \mathrm{~W}$ 的功率 升级。因此, 所剩下的选择只有光纤放大器和晶体放大器。IPG 公司的 $50 \mathrm{~W}$ 光 纤激光放大器是目前市场上比较可靠的选择之一, 经过调研发现其主要缺点有:

1. 不稳定, 长则 2-3 天短至几个小时, 系统崩溃需要重启。 
2. 不能承受高的峰值功率, 其使用的放大光纤是单模光纤而非大模面积光纤, 因此无法工作在激光脉冲形式下。

3. 光纤具有比较宽的增益波长范围, 即使输入谱很窄, 无法避免光纤增益波长 范围内的自发辐射发生，不利于保持激光倍频效率。

以上问题不会出现在晶体放大器的情况下，一般的晶体都具有很高的损伤阈 值, 能长期工作在稳定状态下。以 $\mathrm{Nd}$ : YVO4 晶体为例, 其增益波长范围为 $0.96 \mathrm{~nm}$, 小于倍频晶体的频谱接受度。下面是增益波长在 $1064 \mathrm{~nm}$ 附近的晶体参 数比较

表格 7.7 放大器晶体参数比较

\begin{tabular}{|c|c|c|c|c|c|c|}
\hline Laser crystal & $\begin{array}{l}\text { Nd doped } \\
\text { (atm \%) }\end{array}$ & $\sigma \quad\left(10^{-19} \mathrm{~cm}^{2}\right)$ & $\alpha\left(\mathrm{cm}^{-1}\right)$ & $\mathrm{t}(\mathrm{ms})$ & $\mathrm{P}_{\mathrm{th}}(\mathrm{mW})$ & $h_{p}(\%)$ \\
\hline \multirow{2}{*}{ Nd:YVO4(a-cut) } & 1.1 & \multirow{2}{*}{25} & 31.2 & 90 & \multirow{2}{*}{78} & \multirow{2}{*}{48.6} \\
\hline & 2 & & 72.4 & 50 & & \\
\hline Nd:YVO4(c-cut) & 1.1 & 7 & 9.2 & 90 & 231 & 45.5 \\
\hline Nd:YAG & 0.85 & 6 & 7.1 & 230 & 115 & 38.6 \\
\hline
\end{tabular}

$\mathrm{Nd}$ : YVO4 晶体在 $1.064 \mathrm{um}$ 和 1.3um 处都具有比较大的受激辐射截面 $\sigma$, a-cut 的 Nd: YVO4 的受激辐射截面是 Nd: YAG 晶体的 4 倍。 $\alpha$ 是晶体对泵浦光的吸 收系数, $\tau$ 是晶体激发态的寿命, $\mathrm{P}_{\mathrm{th}}$ 是晶体的阈值功率, $\mathrm{h}_{\mathrm{p}}$ 是晶体的量子泵浦 效率。综合考虑, 我们确定使用 a-cut 的 Nd: YVO4 晶体作为我们的放大介质 [134]。下面是围绕该晶体的放大器设计蓝图及其他元件介绍。

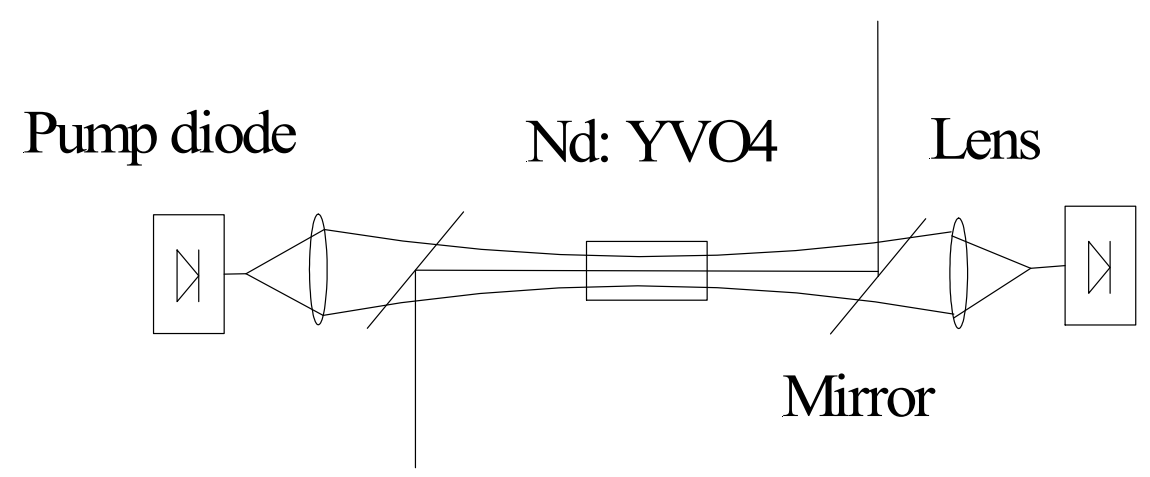

图 7.37 固体放大器设置示意图 
出于模式匹配的考虑, 该放大器采用了两端洜浦的结构。透镜的设计将基于 输出洜浦光的品质将其束腰聚焦到晶体的中心。反射镜镀膜的要求是传输泵浦光 反射被放大的激光。

可用于 $\mathrm{Nd}$ ：YVO4 晶体的泵浦光波长有 $808 \mathrm{~nm}$ 和 $888 \mathrm{~nm}$, 下面给出的是该 晶体的吸收谱, $\mathrm{a}$ 和 $\mathrm{c}$ 分别代表晶体对这两个方向极化光的吸收。一方面, 我们 希望晶体对非极化的泵浦光极化分量均匀吸收; 另外, 小的吸收系数可以保证在 晶体纵向上泵浦光的均匀吸收, 从而减小晶体局部的热效应。所以我们选择了吸 收系数相对小的 $888 \mathrm{~nm}$ 作为葲浦光源 [135]。

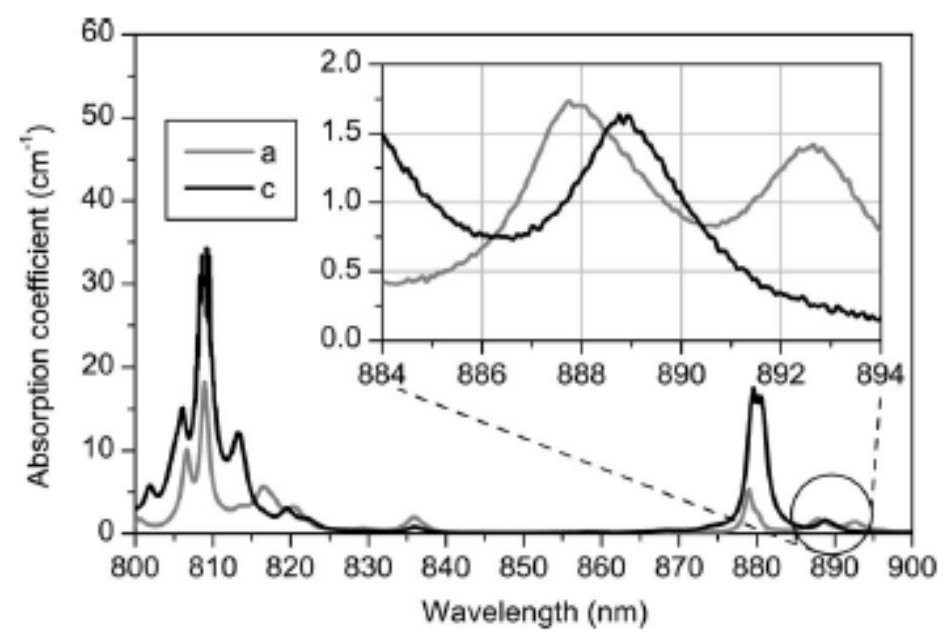

图 7.38 Nd: YVO4 晶体吸收特性曲线

\section{5 本章小结}

本章重点介绍了增益开关二极管技术和光纤技术，详细介绍了 $5 \mathrm{~W}$ 激光系统 的各个部件, 以及在该系统上所进行的一系列测量实验和两项新的技术一一脉冲 选择和脉冲生成技术。为康普顿电子极化仪的激光系统打下了很好的基础, 并对 $50 \mathrm{~W}$ 高功率系统的性能进行了计算, 提出了晶体放大器的设计方案。 


\section{第八章 总结}

本论文的工作致力于发展新型的非拦截式束流诊断方法, 分别进行了 ODR 束流束斑大小测量的实验研究和搭建了用于康普顿电子极化仪的低功率激光系 统。作者在这两个项目的研究过程中, 掌握了电子束辐射测量的相关技术, 并发 展了匹配的分析处理软件, 积累了对辐射测量的分析经验; 并且, 获得了相当的 激光光学的实际经验, 掌握了激光参数测量的基本手段, 通过结合增益二极管激 光技术和光纤技术，搭建了完整的低功率激光系统。

论文第一章介绍了现代加速器应用对测量系统的要求, 从而引申出发展非拦 截测量方法的必要性和紧迫性, 说明了本论文工作的意义。同时也介绍了已经开 展的一系列工作，指出了束流诊断今后发展的方向。

论文第二章总结了衍射辐射的基本理论, 通过模拟计算对单边衍射辐射的强 度分布图案进行了分析, 为后面的实际测量提供了很好的理论指导。并且讨论了 辐射测量中的精度以及提高精度的方法。

论文第三章介绍了衍射辐射测量的实验装置, 各个元件的参数及选择标准, 提供了比较清晰的测量方案。

论文第四章按照测量的实际过程详细介绍了实验测量的准备工作, 以及给出 了相关测量的实验数据。

论文第五章给出了实验数据的分析。首先介绍了数据处理的软件和方法; 然 后分析了测量结果和理论预期的差异, 给出了合理的解释并提供了消除外部影响 的解决办法; 通过 OTR 和坚琴测量的比较, 确定了衍射辐射测量的定标办法; 最后分析得到了衍射辐射图像大小和实际束斑大小的线性比例关系。

第六章主要介绍了康普顿电子极化仪的工作方式, 以及其对激光系统的参数 要求, 然后介绍了激光方面的新技术发展和论文中激光系统的总体设置选择。

第七章详细解释了激光系统每一部分的工作原理, 可供选择的技术和选择的 标准。我们选择了增益开关二极管技术作为系统的激光源, 并且特意使用了光纤 耦合的二极管来保持好的激光品质, 使用了 $5 \mathrm{~W}$ 的光纤放大器来增加激光功率, 然后通过非临界匹配的 LBO 倍频晶体来得到 $532 \mathrm{~nm}$ 的绿光。最后通过计算对 $5 \mathrm{~W}$ 激光系统的实验结果进行了验证和 $50 \mathrm{~W}$ 的激光系统性能进行了评估, 通过对光 
纤放大器和晶体放大器的比较确定了使用晶体放大器的方案, 给出了 $50 \mathrm{~W}$ 晶体 放大器的设计蓝图。

本论文在衍射辐射测量方面工作的意义在于: 验证了衍射辐射束斑大小测量 的可靠性, 相比于 APS 实验得到的衍射辐射图像大小和实际束斑大小的随机关 系, 本实验得到的线性关系对衍射辐射测量起到了很大的推动作用; 衍射辐射测 量的成功为 CEBAF 机器提供了一个非拦截束斑测量方法, 推动了对 CEBAF 机 器的束流测量的更新换代; 而且, 该实验探索了衍射辐射测量的适用范围, 为其 在更多加速器上的应用提供了有力的支持, 为其在新一代直线加速器上的应用打 下了坚实的基础。论文在激光系统方面的工作意义在于: 将增益开关二极管技术 和光纤技术引入了激光在加速器上的应用, 该系统的高品质激光和灵活的参数选 择不仅适用于康普顿电子极化仪的应用, 同时也可以用作光阴极驱动激光系统, 论文中所演示的脉冲选择技术和脉冲生成技术改善了激光系统的性能并拓展了 其应用空间。 


\section{参考文献}

1. Minty, M.G. and F. Zimmermann, Measurement and control of charged particle beams. 2003: Springer.

2. $\quad$ Ross, M.C., et al. Wire scanners for beam size and emittance measurements at the SLC. 1991.

3. Graves, W.S. and E.D. Johnson. A high resolution electron beam profile monitor. 1997.

4. Owen, H.L., THE 4TH GENERATION LIGHT SOURCE AT DARESBURY.

5. Adderley, P., et al., The Control and Diagnostics System for the CEBAF Injector. Linac'88, Williamsburg, VA (US), 1988, 1988.

6. Fulton, R., et al., A high resolution wire scanner for micron-size profile measurements at the SLC. Nuclear Instruments and Methods in Physics Research Section A: Accelerators, Spectrometers, Detectors and Associated Equipment, 1989. 274(1-2): p. 37-44.

7. Buon, J., et al. The Orsay spot size monitor for the final focus test beam. 1993.

8. Sakai, H., et al., Measurement of an electron beam size with a laser wire beam profile monitor. Phys Rev S T A B. 4: p. 022801.

9. Jorda, J.P., et al., A Fabry perot cavity for Compton polarimetry. Nuclear Instruments and Methods in Physics Research A, 1998. 412: p. 1-18.

10. Shintake, T., et al. Experiments of nanometer spot size monitor at FFTB using laserinterferometry. 1995.

11. Ross, M. Laser-based profile monitor for electron Beams.

12. Scarpine, V.E., G.R. Tassotto, and A.H. Lumpkin. OTR imaging of intense 120 GeV protons in the NuMI beamline at fnal. 2007.

13. Ginzburg, V.L. and V.N. Tsytovich, Transition radiation and transition scattering. Physica Scripta, 1982. 2(1): p. 182-191.

14. Ter-Mikaelian, M.L., High-energy electromagnetic processes in condensed media. Interscience tracts on physics and astronomy. 1972, New York,: Wiley-Interscience. ix, 457 p.

15. Xiang, D., et al., Wake of a beam passing through a diffraction radiation target. PHYSICAL REVIEW SPECIAL TOPICS-ACCELERATORS AND BEAMS Phys Rev S T A B, 2008. 11: p. 024001.

16. Xiang, D. and W.H. Huang, Evaluation of transition radiation with wake field theory. Nuclear Inst. and Methods in Physics Research, A, 2005. 553(3): p. 381-387.

17. Ferianis, M. and S. Trieste. Optical techniques in beam diagnostics.

18. Hafizi, B. and P. Sprangle, Diffraction effects in directed radiation beams. Journal of the Optical Society of America A, 1991. 8(5): p. 705-717.

19. Fiorito, R.B., D.W. Rule, and W.D. Kimura. Noninvasive beam position, size, divergence and energy diagnostics using diffraction radiation. 1999.

20. Castellano, M., et al., Measurements of coherent diffraction radiation and its application for bunch length diagnostics in particle accelerators. Physical Review E, 2001. 63(5): p. 56501.

21. Lumpkin, A.H., N.S. Sereno, and D.W. Rule, First measurements of subpicosecond electron beam structure by autocorrelation of coherent diffraction radiation. Nuclear Inst. and Methods in Physics Research, A, 2001. 475(1-3): p. 470-475.

22. Fiorito, R.B., et al., Interference of diffraction and transition radiation and its application as a beam divergence diagnostic. Phys Rev S T A B. 9: p. 052802. 
23. Shibata, Y., et al., Observation of coherent diffraction radiation from bunched electrons passing through a circular aperture in the millimeter-and submillimeter-wavelength regions. Physical Review E, 1995. 52(6): p. 6787-6794.

24. Muto, T., et al., Observation of Incoherent Diffraction Radiation from a Single-Edge Target in the Visible-Light Region. Physical Review Letters, 2003. 90(10): p. 104801.

25. Karataev, P., et al., Beam-Size Measurement with Optical Diffraction Radiation at KEK Accelerator Test Facility. Physical Review Letters, 2004. 93(24): p. 244802.

26. Karataev, P., et al., Observation of optical diffraction radiation from a slit target at KEK accelerator test facility. Nuclear Inst. and Methods in Physics Research, B, 2005. 227(1-2): p. 158-169.

27. Muto, T. First Stage Experiment on Optical Diffraction Radiation at KEK-ATF. 2001.

28. Potylitsyn, A.P., Transition radiation and diffraction radiation. Similarities and differences. Nuclear Instruments and Methods in Physics Research Section B, 1998. 145(1-2): p. 169-179.

29. Karataev, P., et al., Status of optical diffraction radiation experiment at KEK-ATF extraction line. Nuclear Inst. and Methods in Physics Research, B, 2003. 201(1): p. 140-152.

30. Naumenko, G.A., Some features of diffraction and transition radiation at the distance less than ?2? Nuclear Inst. and Methods in Physics Research, B, 2005. 227(1-2): p. 87-94.

31. Naumenko, G., Synchrotron radiation contribution to optical diffraction radiation measurements. Nucl. Inst. and Meth. B, 2003. 201: p. 184-190.

32. Shkvarunets, A.G., R.B. Fiorito, and P.G. O 扴 hea, Optical diffraction 杢 ransition radiation interferometry and its application to the measurement of beam divergence. Nuclear Inst. and Methods in Physics Research, B, 2003. 201(1): p. 153-160.

33. Wang, X.J. and H.G. Kirk. The Brookhaven ATF low-emittance beam line. 1991.

34. Lumpkin, A.H., et al., Near-field imaging of optical diffraction radiation generated by a 7-GeV electron beam. Phys Rev S T A B. 10: p. 022802.

35. Lumpkin, A.H., First Near-Field Imaging of Optical Diffraction Radiation Generated by a 7-GeV Electron Beam. submitted to Phys. Rev. Lett, 2005.

36. Castellano, M., et al., Measurements of coherent diffraction radiation and its application for bunch length diagnostics in particle accelerators. Physical Review E, 2001. 63(5).

37. Martin, D.H., Polarizing (Martin-Puplett) interferometric spectrometers for the near-and submillimeter spectra. Infrared and millimeter waves, 1982. 6: p. 65-148.

38. Castellano, M., Diffraction Radiation as a Diagnostics Tool at FLASH. 2008, INFN-LNF: Roma.

39. Leemann, C.W., D.R. Douglas, and G.A. Krafft, T HE C ONTINUOUS E LECTRON B EAM A CCELERATOR F ACILITY: CEBAF at the Jefferson Laboratory. Annual Review of Nuclear and Particle Science, 2001. 51(1): p. 413-450.

40. Krafft, G.A. Status of the Continuous Electron Beam Accelerator Facility. 1994.

41. Lumpkin, A.H., et al., Feasibility of near-field ODR imaging of multi-GeV electron beams at CEBAF. Energy (GeV). 7(5): p. 5-250.

42. Borland, M., et al. Configuration, optics, and performance of a 7-GEV Energy Recovery Linac upgrade for the advanced photon source. 2007.

43. Loew, G.A., S.L.A. Center, and C.A. Menlo Park. Report from the international linear collider technical review committee. 2003.

44. Aarons, G., International linear collider reference design report. 2007, LBNL--62867, 


\section{COLLABORATION-ILCGDE.}

45. Roos, P.G., The GO experiment: Parity violation in eN elastic scattering. The European Physical Journal A-Hadrons and Nuclei, 2005. 24: p. 59-63.

46. Jackson, J.D., Classical electrodynamics. 3rd ed. 1999, New York: Wiley. xxi, 808 p.

47. Einstein, A., The meaning of relativity. 1956, London: Methuen. $161 \mathrm{p}$.

48. Bolotovskii, B.M. and G.V. Voskresenskii, Diffraction radiation. Physics-Uspekhi, 1966. 9(1): p. 73-96.

49. Lumpkin, A.H., et al. Nonintercepting Electron Beam Diagnostics Based on Optical Diffraction Radiation for X-ray FELs. 2005.

50. Yunn, B.C. Physics of JLab FEL injector. 1999.

51. Lebedev, V., OptiM-Computer code for linear and non-linear optics calculations.

52. Yao, C.Y., et al. Numerical simulation of optical diffraction radiation from A 7-GeV beam. 2007.

53. Hofmann, A. and F. Meot, Optical resolution of beam crosssection measurements by means of synchrotron radiation. Nucl. Instr. and Meth, 1982. 203: p. 483.

54. Castellano, M. and V.A. Verzilov, Spatial resolution in optical transition radiation beam diagnostics. Physical Review Special Topics- Accelerators and Beams, 1998. 1(6): p. 50.

55. Artru, X., et al., Experimental investigations on geometrical resolution of optical transition radiation (otr). Nuclear Instruments and Methods in Physics Research Section A: Accelerators, Spectrometers, Detectors and Associated Equipment, 1998. 410(2): p. 148-158.

56. Lebedev, V.A., Diffraction-limited resolution of the optical transition radiation monitor. Nuclear instruments \& methods in physics research. Section A, Accelerators, spectrometers, detectors and associated equipment, 1996. 372(3): p. 344-348.

57. Xiang, D. and W.H. Huang, Theoretical considerations on imaging of micron size electron beam with optical transition radiation. Nuclear Inst. and Methods in Physics Research, A, 2007. 570(3): p. 357-364.

58. Fiorito, R.B., et al. OTR measurements of the $10 \mathrm{keV}$ electron beam at the University of Maryland Electron Ring (UMER).

59. Catravas, P., et al. Beam profile measurement at $30 \mathrm{GeV}$ using optical transitionradiation. 1999.

60. Shafer, D.R., Laser beam expander. 1980, Google Patents.

61. Hodgson, N., et al., Determination of laser beam parameters with the phase space beam analyser. Optical and Quantum Electronics, 1992. 24(9): p. 927-949.

62. P. Evtushenko, A.P.F., C. Y. Liu, A. Lumpkin, Near-field Optical Diffraction Radiation Measurements at CEBAF, in BIW08. 2008.

63. Vermare, C., D.C. Moir, and G.J. Seitz, Optical transition radiation (OTR) measurements of an intense pulsed electron beam. Arxiv preprint physics/0008150, 2000.

64. Wkiewicz, K., Classical and quantum Malus laws. Commun. Math. Phys Phys Rev A, 1973. 51: p. 2785.

65. Photonics, R.P., Encyclopedia of Laser Physics and Technology, Superluminescense 2007-12-19, < http://www. rp-photonics.com/superluminescence.html>.

66. Strong, J., Achromatic Doublet Lenses for Infrared Radiation. Applied Optics, 1971. 10(6): p. 1439-1443.

67. Holst, G.C., CCD arrays, cameras, and displays. 
68. Scanner, E.R.W., Large Dynamic Range Transverse Beam Profile with Wire Scanners.

69. Abbott, R., B. Bowling, and E. Woodworth, HARP User's Manual. CEBAF Tech Note TN92-050, 1992.

70. $\quad$ Private communication with A. P. Freyberger and M. Tiefenback

71. Woods, A., T. Docherty, and R. Koch, Image distortions in stereoscopic video systems. Stereoscopic displays and applications IV: p. 36-48.

72. Ojanen, H., Automatic correction of lens distortion by using digital image processing. Rutgers University, Dept. of Mathematics technical report, 1999.

73. Bennett, H.F., TELECENTBIC LENS SYSTEM. 1945, Google Patents.

74. Dietzsch, C. and E. Dietzsch, Telecentric relay lens system. 1997, Google Patents.

75. Roblin, Y. and A. Freyberger, Studies of Beam Halo Formation in the 12 GeV CEBAF Design. 2007.

76. Christiansen, T. and N. Torkington, Perl cookbook. 1998: O'Reilly \& Associates, Inc. Sebastopol, CA, USA.

77. Westfold, K.C., The Polarization of Synchrotron Radiation. The Astrophysical Journal, 1959. 130: p. 241.

78. Jackson, J.D. and R.F. Fox, Classical electrodynamics. American Journal of Physics, 1999. 67: p. 841 .

79. Bosch, R.A., Long-wavelength edge radiation along a straight-section axis in an electron storage ring. Nucl. Instr. and Meth, 1997. 386: p. 525-530.

80. Bosch, R.A., et al., Infrared radiation from bending magnet edges in an electron storage ring. Review of Scientific Instruments, 1996. 67: p. 3346.

81. Schade, U., et al., Measured characteristics of infrared edge radiation from BESSY II. Nuclear Inst. and Methods in Physics Research, A, 2000. 455(2): p. 476-486.

82. Geloni, G., et al., Theory of edge radiation. Part I: Foundations and basic applications. Nuclear Instruments and Methods in Physics Research Section A: Accelerators, Spectrometers, Detectors and Associated Equipment.

83. Bosch, R.A., Computed flux and brightness of infrared edge and synchrotron radiation. Nuclear Instruments and Methods in Physics Research Section A: Accelerators, Spectrometers, Detectors and Associated Equipment, 2000. 454(2-3): p. 497-505.

84. Chubar, O., P. Elleaume, and A. Snigirev, Phase analysis and focusing of synchrotron radiation. Nuclear Instruments and Methods in Physics Research Section A: Accelerators, Spectrometers, Detectors and Associated Equipment, 1999. 435(3): p. 495-508.

85. Margenau, H. and G.M. Murphy, The mathematics of physics and chemistry. 1956: New York.

86. Travier, C., An introduction to photo-injector design. Nuclear Instruments and Methods in Physics Research-Section A Only, 1994. 340(1): p. 26-39.

87. Palmer, D.T., The next generation photoinjector. PhD Manuscript, June, 1998.

88. Xiang, R., et al., Experimental investigations of DC-SC photoinjector at Peking University. Nuclear Inst. and Methods in Physics Research, A, 2004. 528(1-2): p. 321-325.

89. Luiten, O.J., et al., How to realize uniform three-dimensional ellipsoidal electron bunches. IEEE Trans. Magn Phys Rev Lett, 2004. 93: p. 094802.

90. Zhou, S., et al., Efficient temporal shaping of ultrashort pulses with birefringent crystals. Applied Optics, 2007. 46(35): p. 8488-8492.

91. Zhang, S., G. Neil, and M. Shinn, Single-element laser beam shaper for uniform flat-top 
profiles. Optics Express, 2003. 11(16): p. 1942-1948.

92. Yang, J., et al., Low-emittance electron-beam generation with laser pulse shaping in photocathode radio-frequency gun. Journal of Applied Physics, 2002. 92: p. 1608.

93. Cialdi, S. and I. Boscolo, A laser pulse shaper for the low-emittance radiofrequency SPARC electron gun. Nuclear Inst. and Methods in Physics Research, A, 2004. 526(3): p. 239-248.

94. Liu, C. and S. Zhang, Study of singular radius and surface boundary constraints in refractive beam shaper design. Optics Express, 2008. 16(9): p. 6675-6682.

95. Van Tilborg, J., et al., Temporal characterization of femtosecond laser-plasma-accelerated electron bunches using terahertz radiation. laser Phys Rev Lett. 96: p. 014801.

96. Casalbuoni, S., et al. Numerical studies on the electro-optic sampling of relativistic electron bunches. 2005.

97. Kashiwagi, S., et al., Observation of high-intensity X-rays in inverse Compton scattering experiment. Nuclear Inst. and Methods in Physics Research, A, 2000. 455(1): p. 36-40.

98. Carroll, F.E., et al., Pulsed tunable monochromatic x-ray beams from a compact source: new opportunities. 2003, Am Roentgen Ray Soc. p. 1197-1202.

99. Andruszkow, J., First observation of self-amplified spontaneous emission in a free-electron laser at $109 \mathrm{~nm}$ wavelength. Arxiv preprint physics/0006010, 2000.

100. Pellegrini, C., Free Electron Lasers. Development and Applications of Free Electron Lasers, 1997: p. 133.

101. Zhao, K., et al., Peking university superconducting accelerator facility for free electron laser. Nuclear Inst. and Methods in Physics Research, A, 2002. 483(1-2): p. 125-128.

102. 丁原涛, 北京大学 SASE FEL 的物理研究与优化设计. 2004, 北京大学.

103. Faure, J., et al., A laser 杙 lasma accelerator producing monoenergetic electron beams. Nature, 2004. 431: p. 541-544.

104. Tajima, T., High energy laser plasma accelerators. Laser and Particle Beams, 1985. 3: p. 351-413.

105. Eisenberger, P. and P.M. Platzman, Compton Scattering of $X$ Rays from Bound Electrons. Physical Review A, 1970. 2(2): p. 415-423.

106. Jones, F.C., Calculated Spectrum of Inverse-Compton-Scattered Photons. Nature Phys Rev, 1966. 167: p. 1159.

107. Bardin, G., et al., Conceptual design report of a Compton polarimeter for CEBAF Hall A. DAPNIA/SPhN/96-14 p. 65.

108. Giesekus, J., et al., High power diode end pumped slab MOPA system.

109. Siegman, A.E., Lasers. 1986, Mill Valley, Calif.: University Science Books. xxii, 1283 p.

110. Demokan, M.S., Mode-locking in solid-state and semiconductor lasers. Electronic \& electrical engineering research studies. Lasers and fibre optics series. 1982, Chichester, England ; New York: Research Studies Press. xi, 227 p.

111. Gaeta, C.J., CW and Q-switched Nd:YAG fiber lasers. 1986, Stanford University, 1986. p. xxv, 211 p., bound.

112. Killi, A., et al., Diode-pumped femtosecond laser oscillator with cavity dumping. Optics letters, 2004. 29(11): p. 1288-1290.

113. Pshenichnikov, M.S., W.P. de Boeij, and D.A. Wiersma, Generation of 13-fs, 5-MW pulses from a cavity-dumped Ti: sapphire laser. Optics letters, 1994. 19(8): p. 572-574.

114. van der Pol, B., On relaxation oscillations. Phil. Mag, 1926. 2(11): p. $978 ? 92$. 
115. Vasil'ev, P.P., Ultrashort pulse generation in diode lasers. Optical and Quantum Electronics, 1992. 24(8): p. 801-824.

116. Arakawa, Y., et al., Picosecond pulse generation (<1.8 ps) in a quantum well laser by a gain switching method. Applied Physics Letters, 1987. 51: p. 1295.

117. Poelker, M., High power gain-switched diode laser master oscillator and amplifier. Applied Physics Letters, 1995. 67: p. 2762.

118. Barry, L.P., et al., Tunable transform-limited pulse generation using self-injection locking of an FP laser. IEEE Photonics Technology Letters, 1993. 5(10): p. 1132-1134.

119. Gunning, P., et al., Gainswitched DFB laser diode pulse source using continuous wavelight injection for jitter suppression and an electroabsorptionmodulator for pedestal suppression. Electronics Letters, 1996. 32(11): p. 1010-1011.

120. http://www.qphotonics.com/product.php?productid $=80 \& c a t=0$.

121. Mukai, T., M. Yamada, and S. Nakamura, Characteristics of InGaN-based UV/blue/green/amber/red light-emitting diodes. Jpn. J. Appl. Phys., Part, 1999. 1(38): p. 3976.

122. Arkhipov, V.I., et al., Charge injection into light-emitting diodes: Theory and experiment. Journal of Applied Physics, 1998. 84: p. 848.

123. Cheville, R.A., M.T. Reiten, and N.J. Halas, Wide-bandwidth frequency doubling with high conversion efficiency. Optics letters, 1992. 17(19): p. 1343.

124. http://www.fiberoptics4sale.com/Merchant2/fiber-optic-connectors.php.

125. Goldsmith, P.F., I.M. Theory, and S. Techniques, Quasioptical systems: Gaussian beam quasioptical propagation and applications. 1998: IEEE Press Piscataway, NJ.

126. Siegman, A.E., G. Nemes, and J. Serna, How to (maybe) measure laser beam quality. OSA TOPS, 1998. 17(2): p. 184-199.

127. Kleinman, D.A., Theory of second harmonic generation of light. J. Appl. Phys Phys Rev, 1961. 128: p. 1761.

128. Hobden, M.V., Phase-matched second-harmonic generation in biaxial crystals. Journal of Applied Physics, 1967. 38: p. 4365.

129. Chen, D.W. and J.T. Lin, Temperature-tuned phase-matching properties ofLiB 3 O 5 for Ti: sapphire laser frequency doubling. IEEE Journal of Quantum Electronics, 1993. 29(2): p. 307-310.

130. Kato, K., Temperature-tuned 90 phase-matching properties of LiB 3 O 5. IEEE Journal of Quantum Electronics, 1994. 30(12): p. 2950-2952.

131. Smith, A.V., SNLO nonlinear optics code. Sandia National Laboratories, Albuquerque, NM, 2004: p. 87185-1423.

132. Smith, A.V. How to select nonlinear crystals and model their performance using SNLO software. 2000.

133. Wang, H. and A.M. Weiner, Efficiency of short-pulse type-I second-harmonic generation with simultaneous spatial walk-off, temporal walk-off, and pump depletion. IEEE Journal of Quantum Electronics, 2003. 39(12): p. 1600-1618.

134. McDonagh, L., R. Wallenstein, and G. Kaiserslautern, Lasers \& Sources Optimized pumping of neodymium-doped vanadate yields high-power lasers.

135. McDonagh, L., et al., High-efficiency 60 W TEM00 Nd:YVO4 oscillator pumped at $888 \mathrm{~nm}$. Opt. Lett., 2006. 31(22): p. 3297-3299. 


\section{致 谢}

本论文的完成得到了北京大学重离子物理研究所和美国Jefferson实验室多位 老师和同学的指导和帮助。

首先感谢我的导师赵竞教授, 感谢他在这六年的时间里给予的学习上的悉心 教导和生活上的关怀, 在许多问题上给予的启迪, 并提供了大量机会, 使我的研 究工作得以顺利进行, 感谢他领导的射频超导组提供的良好实验条件和宽松的工 作环境。赵老师学识洲博, 思维敏锐, 经验丰富, 对科研追求严谨和不断创新的 精神、为人处世的坦荡和豁达使我终身受益, 他的言传身教将是我一生的财富。 我将谨遵恩师的教诲, 孜孜以求, 不断进取, 以不幸负恩师对我的期望。

感谢我在美国Jefferson Lab的指导老师Dr. Geoffrey Krafft, 感谢他对我的论文 工作的总体指导和安排, 为我在Jefferson实验室的学习创造了有益的环境。Dr. Geoffrey Krafft 治学严谨, 知识波博, 诲人不倦以及对工作的热爱和一丝不苟的 态度, 都为我做出了榜样。感谢他对我生活上的关心, 使我很快接受并适应新的 环境, 安心进行论文设计。

感谢Jefferson Lab的Dr. Pavel Evtushenko, Dr. Arne Freyberger和Fermilab的Dr.

A. H. Lumpkin在衍射辐射测量项目中给予的指导, 每一次的讨论都让作者收益 多多, 他们的悉心指导对作者的知识结构和思维方式都产生了巨大的影响, 并将 帮助我的未来科研之路。

感谢Jefferson Lab的Zhang Shukui (张树魁老师), Matt Poelker, Dave Gaskell, John Hansknecht在康普顿激光项目上对我的细心指导, 他们波博的知识、专业精 神和团队精神使作者受益匪浅。特别感谢张树魁老师在科研、生活方面给予的无 私帮助和指引。

感谢北京大学客座教授庄杰佳先生对我论文工作的关心, 庄老师滟博的学 识、丰富的经验和严谨的作风给我留下了深刻的印象。

感谢陈佳洱院士在百忙之中对论文工作的关心和支持, 他严谨的治学态度令 作者终身难忘。感谢鲁向阳老师几年来对作者无私的帮助, 还有他在学习生活、 实验工作和论文写作上提出的大量前瞻性的建议。感谢张保澄教授对论文工作的 支持和帮助, 对论文的完善提出宝贵的意见。感谢全胜文高工和林林老师、丁原 
涛老师、黄森林老师与作者的多次讨论和指导, 感谢王莉芳老师、谢大㢷老师、 郝建奎老师、杨丽敏老师、朱风老师、吕德泉老师六年来在学习和生活上给予我 很多的无私帮助。感谢JLAB给我提供的研究机会。感谢CASA组成员所给予无私 帮助和大量讨论, 感谢Lia Merminga和Geoffrey Krafft 给予工作上的大力支持和 指导。感谢Hari Areti对作者工作和生活的安排。感谢Swapan Chattopadhyay给作 者提供的研究条件。

感谢吴文忠、储祥蓄、焦飞、肖斌平、王芳、刘振超、徐文灿等师兄师姐师 弟师妹们, 感谢他们在学习和生活上给予我的帮助。

最后, 感谢我的妻子王桂梅和我们即将出生的儿子Eric对我的全力支持, 他 们是我完成博士论文的动力源泉; 感谢我的父母家人对我多年的培育, 以及对我 博士论文工作的理解和支持。

论文的完成远非终点, 文中的不足和浅显之处是我新征程的起点, 我将带着 你们的教导和期望继续前行, 努力做一个有用之人。 


\section{北京大学学位论文原创性声明和使用授权说明}

\section{原创性声明}

本人郑重声明: 所呈交的学位论文, 是本人在导师的指导下, 独立进行研 究工作所取得的成果。除文中已经注明引用的内容外, 本论文不含任何其他个人 或集体已经发表或撰写过的作品或成果。对本文的研究做出重要贡献的个人和集 体, 均已在文中以明确方式标明。本声明的法律结果由本人承担。

论文作者签名：日期：日年月日

\section{学位论文使用授权说明}

(必须装订在提交学校图书馆的印刷本)

本人完全了解北京大学关于收集、保存、使用学位论文的规定, 即:

- 按照学校要求提交学位论文的印刷本和电子版本;

- 学校有权保存学位论文的印刷本和电子版, 并提供目录检索与阅览服 务，在校园网上提供服务;

- 学校可以采用影印、缩印、数字化或其它复制手段保存论文;

- 因某种特殊原因需要延迟发布学位论文电子版, 授权学校 $\square$ 一年/ $\square$ 两年 $/ \square$ 三年以后，在校园网上全文发布。

（保密论文在解密后遵守此规定）

论文作者签名:

导师签名：

日期：年月日 